\title{
INDEX OF DATES FROM ARCHAEOLOGICAL SITES IN QUEENSLAND
}

\author{
SEAN ULM AND JILL REID \\ Aboriginal and Torres Strait Islander Studies Unit, University of Queensland, \\ Brisbane, Queensland, 4072, Australia
}

\begin{abstract}
This volume presents an indexed compilation of chronometric determinations obtained from archaeological sites in the state of Queensland (including Torres Strait), Australia, to the end of 2000 . The list includes conventional radiocarbon $\left({ }^{14} \mathrm{C}\right)$, accelerator mass spectrometry (AMS), thermoluminescence (TL) and optically-stimulated luminescence (OSL) determinations. In total, 849 dates are listed from 258 sites. This listing is intended as a reference work only and no analysis of the dataset is undertaken in this volume.
\end{abstract}

\section{Introduction}

In 1982, Michael Kelly compiled and published a list of radiocarbon and thermoluminescence dates obtained from archaeological sites in Queensland entitled A Practical Reference Source to Radiocarbon Dates Obtained from Archaeological Sites in Queensland. This monograph listed 164 radiocarbon dates and two thermoluminescence dates from 69 sites. Kelly's desire that "the radiocarbon list begun here will maintain pace with the burgeoning growth of archaeological investigation in Queensland" (1982:9) was never realised with very limited continuing input from archaeologists and no subsequent updates published.

In the 18 years that have elapsed since that compilation the pace of archaeological research in Queensland has increased dramatically (Figure 1). Large chronometric datasets have been generated by projects in Cape York Peninsula (e.g. Cole 1998; David 1998; David and Chant 1995; Morwood and Hobbs 1995), southwest Queensland (Robins 1995), central Queensland (e.g. Barker 1995; Border 1999; Lilley and Ulm 1995, 1999; Lilley et al. 1999b; Morwood 1984) and southeast Queensland (e.g. McNiven 1990a; Ulm and Hall 1996). Although several regional syntheses have emerged (e.g. David and Lourandos 1997; Ulm and Hall 1996), no work has attempted to re-synthesise this burgeoning database. In the absence of other sources, Kelly's (1982) monograph has become a unique resource for archaeologists working in Queensland which has no equivalent elsewhere in Australia (cf. Flood 1999:283-289; Stockton 1981).

The Index of Dates from Archaeological Sites in Queensland attempts to build on Kelly's (1982) work and to expand its utility by including indexes and key maps to facilitate various search options. The Index has three primary aims. The first is to provide a comprehensive listing of chronometric determinations obtained from archaeological sites in Queensland, including sample provenance and sample activity data where available.

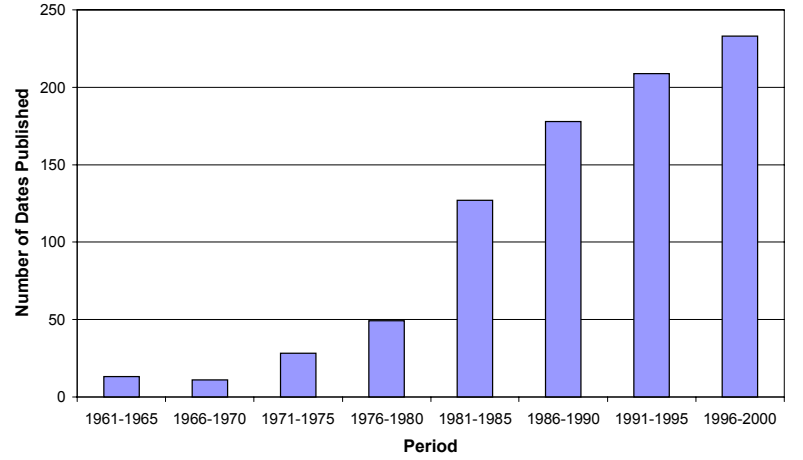

Figure 1. Rate of publication of chronometric dates from archaeological sites in Queensland in five-year intervals from 1961.

Secondly it aims to present basic information about chronometrically-dated sites in Queensland, including site location and basic site characteristics. The third aim is to provide a list of references for each of the dated sites which covers excavation, laboratory analysis, dating and interpretation. This last component forms a type of citation index and provides a structured entry-point to the literature concerning individual sites or sites in a particular region. The Index should be considered as a reference source only and not a substitute for the references cited.

The Index was compiled and structured with the needs of a number of potential user-groups in mind. Foremost amongst these are Aboriginal and Torres Strait Islander communities, which often experience difficulties accessing the results of archaeological research conducted in their country. The references which appear in Section 11 highlight the fact that virtually all of the information presented is derived from widely-scattered professional journals and unpublished theses which are often difficult for university-based academics to access, let alone rural or remote communities. Cultural heritage consultants and local government agencies will find the Index useful for quickly identifying whether dated archaeological sites occur in a specific management 
or potential development area. Additionally, the sitespecific references for given areas provide an entrypoint to the literature on recorded but undated sites in a given region. Academics and other researchers may use the Index as a tool in analyses of site data or as an efficient entry-point to the literature. Finally, radiocarbon specialists will find technical details of sample activities and sample types useful in studies evaluating the efficacy of dating specific sample materials.

\section{Methods}

The Index was compiled through a survey of published sources, unpublished reports and postgraduate research theses as well as directly from public lists published by radiocarbon laboratories. This search involved systematically reviewing issues of Queensland Archaeological Research, Australian Archaeology, Archaeology in Oceania and Radiocarbon and relevant theses held at the University of Queensland, the University of New England, James Cook University of North Queensland, the University of Sydney and the Australian National University. Many theses were identified using White's $(1975,1994)$ compilations of Australian archaeological postgraduate research theses.

In addition to Kelly (1982), which was used as a starting point, a number of regional syntheses of archaeological datasets were used as an entry point to the literature containing chronometric dates: Ulm and Hall (1996) for southeast Queensland; Lilley et al. (1999b) and Rowland (1996) for the central Queensland coast; Morwood (1984) for the Central Queensland Highlands; David et al. (1998), David et al. (1999), David and Lourandos (1997) and David and Wilson (1999) for southeast Cape York Peninsula; and Barham et al. (in prep.) for Torres Strait. Wherever possible, original sources were consulted to minimize the potential for reporting error in secondary compilations. Where discrepancies were identified between determinations published in multiple sources every effort was made to clarify the error. Where this was not possible, the discrepancy was noted.

All dates listed have either been presented in a published form (e.g. journal article, book, postgraduate research thesis, World Wide Web, consultancy report) or are included with the express permission of the principal investigator of the site in question. On this basis, some determinations which were presented at conferences or that are in press or otherwise not yet published were not available for inclusion (see below). Conservatively, we estimate that this listing represents over $90 \%$ of determinations made at archaeological sites in Queensland.
In addition, individual researchers were contacted to confirm details of determinations, supply missing information where required and to seek access to otherwise unavailable data. In total, this search resulted in the identification of 849 dates from 258 sites, including several dates which were not previously reported (Figure 2, Table 1).

\section{Structure of the Index}

The Index has been structured with the needs of various user groups in mind. Determination details, site data and indexes have been divided into a number of sections outlined below to facilitate ease of access. In Section 1 we have grouped the basic data concerning each determination together in anticipation that this would be the most heavily used part of the Index. Other details are presented in subsequent sections.

\section{Section 1: Basic Determination Data}

This section lists basic determination data for all chronometric dates available from archaeological sites in Queensland. Basic data include site name, laboratory code, dating method $\left({ }^{14} \mathrm{C}, \mathrm{AMS}\right.$, TL or OSL), the reported age (the conventional radiocarbon age in the case of ${ }^{14} \mathrm{C}$ and AMS determinations and the published age for TL and OSL dates), standard error, calibrated age/s for radiocarbon dates, sample material and earliest reference for the date information. See Explanatory Notes below for a full description of field data.

\section{Section 2: Advanced Determination Data}

Technical data on each determination is given (where available), including the sample species, weight of the sample submitted to the laboratory (as distinct to the weight of the sample after pretreatment), sample provenience (square, excavation unit, depth below surface), and, for radiocarbon dates, d14C, d13C, D14C and \%Modern values. See Explanatory Notes below for a full description of field data.

\section{Section 3: Notes on Individual Determinations}

This section includes comments on individual determinations which require further explanation or clarification. Comments are made, for example, where dates or sample details are reported differently in multiple publications or where there is a potential problem with a particular piece of data associated with a date. Comments of investigators are also included where dates are considered anomalous or otherwise problematic. Note that not all determinations have associated comments. Determinations with accompanying notes are marked by shading of the laboratory code in Sections 1-2 (above). 


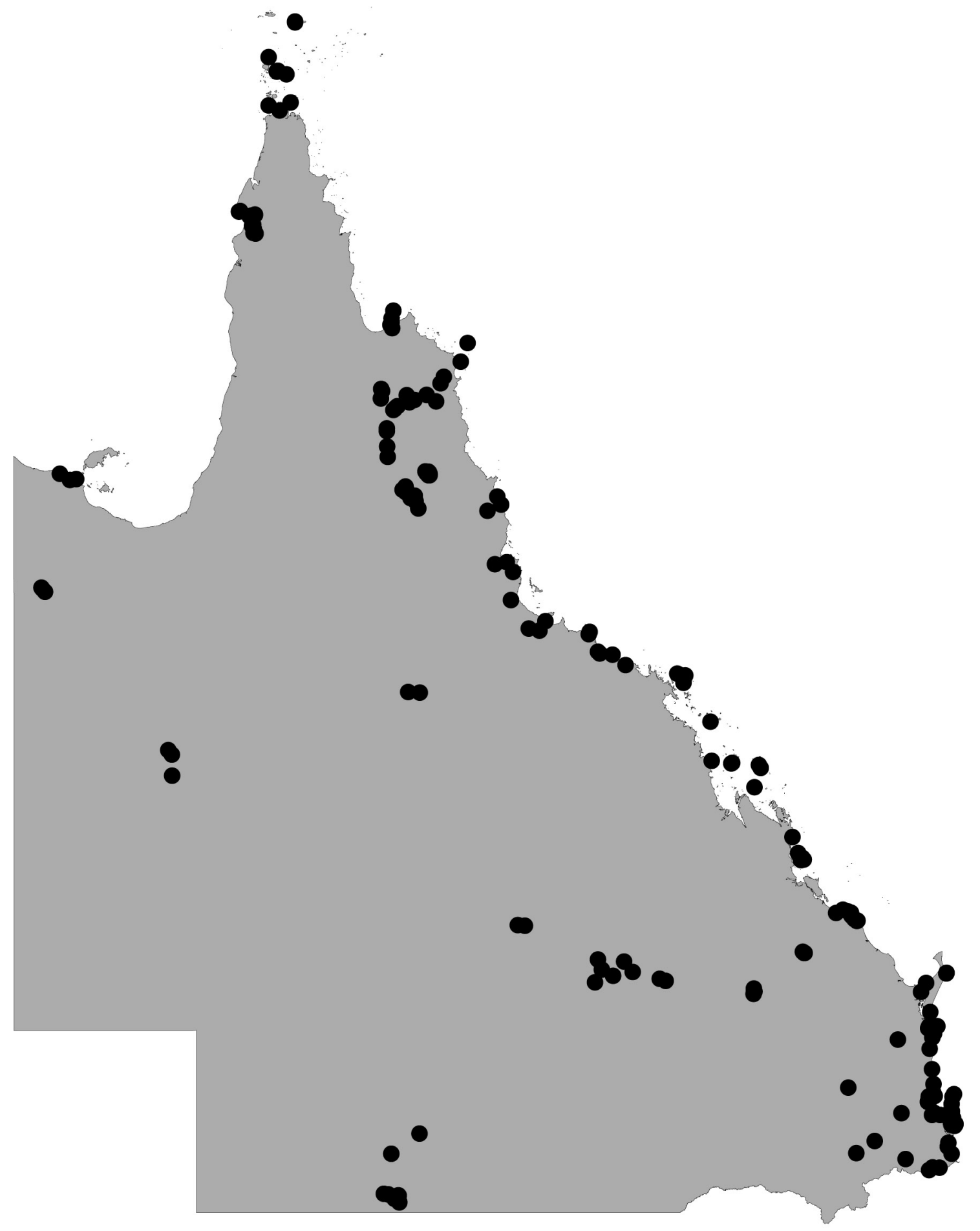

Figure 2. Distribution of dated archaeological sites in Queensland included in the Index. Note that five sites (PCB 13, PCB 111, PCB 141, Red Lady Rock Art and Shelly Beach) included in the Index do not appear in the figure as they do not have geographical coordinates available. Sites listed in Table 2 are also not shown. 
Table 1. Summary of the number of dates and number of dated sites by 1:250,000 map sheet in descending order.

\begin{tabular}{|c|c|}
\hline 1:250K Sheet & \# of Dates \\
\hline Mossman & 101 \\
\hline Atherton & 95 \\
\hline Brisbane & 81 \\
\hline Cooktown & 75 \\
\hline Weipa & 58 \\
\hline Bundaberg & 53 \\
\hline Cape Melville & 43 \\
\hline Monto & 25 \\
\hline Springsure & 23 \\
\hline Rockhampton & 22 \\
\hline Tweed Heads & 22 \\
\hline Eddystone & 18 \\
\hline Eulo & 18 \\
\hline Gympie & 17 \\
\hline Innisfail & 17 \\
\hline Lawn Hill & 17 \\
\hline Duchess & 16 \\
\hline Warwick & 14 \\
\hline Ayr & 13 \\
\hline Hughenden & 10 \\
\hline Proserpine & 10 \\
\hline Torres Strait & 10 \\
\hline Wide Bay & 10 \\
\hline Maryborough & 9 \\
\hline Ipswich & 8 \\
\hline Cairns & 7 \\
\hline Ingham & 7 \\
\hline Mornington & 7 \\
\hline Townsville & 7 \\
\hline Mundubbera & 5 \\
\hline Percy Isles & 5 \\
\hline Toompine & 5 \\
\hline Daru & 4 \\
\hline Mackay & 4 \\
\hline Tambo & 4 \\
\hline Boigu & 3 \\
\hline Taroom & 3 \\
\hline Port Clinton & 1 \\
\hline Unknown & 2 \\
\hline
\end{tabular}

\begin{tabular}{|c|c|}
\hline 1:250K Sheet & \# of Dated Sites \\
\hline Weipa & 37 \\
\hline Brisbane & 31 \\
\hline Mossman & 19 \\
\hline Cooktown & 17 \\
\hline Cape Melville & 11 \\
\hline Atherton & 10 \\
\hline Ayr & 10 \\
\hline Bundaberg & 9 \\
\hline Eulo & 9 \\
\hline Gympie & 9 \\
\hline Rockhampton & 8 \\
\hline Wide Bay & 8 \\
\hline Torres Strait & 7 \\
\hline Eddystone & 5 \\
\hline Monto & 5 \\
\hline Proserpine & 5 \\
\hline Hughenden & 4 \\
\hline Ingham & 4 \\
\hline Mundubbera & 4 \\
\hline Tweed Heads & 4 \\
\hline Duchess & 3 \\
\hline Lawn Hill & 3 \\
\hline Mackay & 3 \\
\hline Maryborough & 3 \\
\hline Mornington & 3 \\
\hline Percy Isles & 3 \\
\hline Townsville & 3 \\
\hline Cairns & 2 \\
\hline Daru & 2 \\
\hline Innisfail & 2 \\
\hline Ipswich & 2 \\
\hline Springsure & 2 \\
\hline Tambo & 2 \\
\hline Taroom & 2 \\
\hline Warwick & 2 \\
\hline Boigu & 1 \\
\hline Port Clinton & 1 \\
\hline Toompine & 1 \\
\hline Unknown & 2 \\
\hline
\end{tabular}




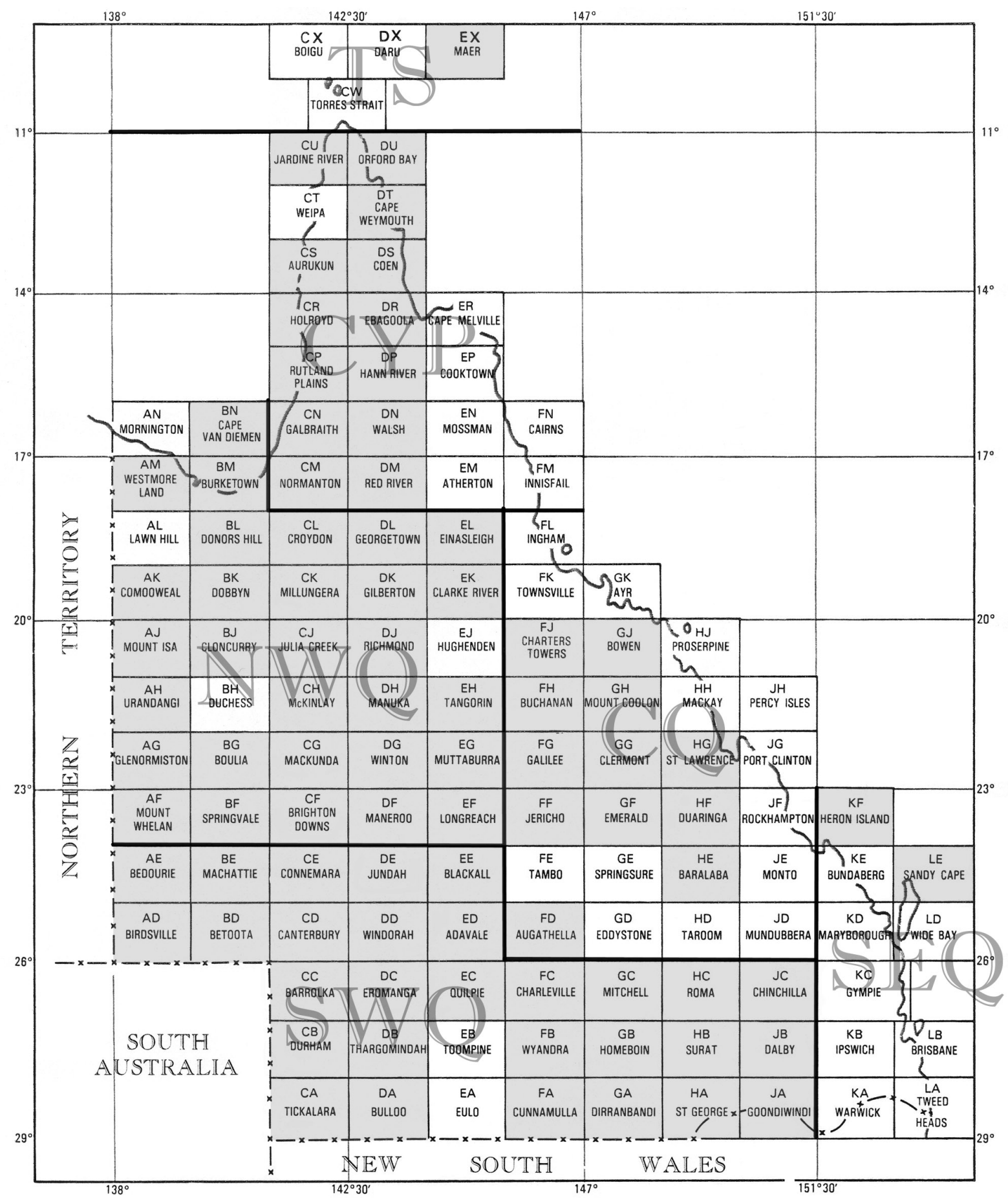

Figure 3. 1:250,000 map sheets with superimposed regions used to structure geographical information in the Index (after Johnston and Rowland 1987). Shaded squares indicate that no dated archaeological sites occur on that map sheet. The two letter code above each map sheet name is used by the Queensland Environmental Protection Agency to code cultural heritage places occurring on that map sheet. 


\section{Section 4: Site Information}

In this section, basic information for each site is presented including: region, 1:250,000 map sheet name, geographical coordinates (decimal latitude and decimal longitude), state site designation, site type (open or rockshelter), whether the site is coastal $(<1 \mathrm{~km}$ from the coast) or inland and the principal investigator/s of the site. See the Explanatory Notes below for a full description of field data.

\section{Section 5: Site References}

This section presents bibliographic sources which refer specifically to the listed site. References cover excavation, laboratory analysis, dating and interpretation. Full bibliographic details are presented in Section 11.

\section{Section 6: Index of Sites by Principal Investigator}

This index lists dated site names by principal investigator. Some sites are listed against more than one principal investigator where sites have been excavated under the direction of more than one person. The principal investigator is defined as the individual who took primary responsibility for the excavation. Note that this index excludes principal investigators who may have excavated at a site but did not actually obtain chronometric determination/s. This index can be used to ascertain which sites in the Index have been investigated by a particular person prior to referring to Sections 1-5 for further details.

Section 7: Index of Sites by Alternative Site Name Some sites are known in the literature by more than one name. This index provides a list of alternative published designations for sites included in the Index. If the name of a dated site is known but it is not listed in the Index it may be listed under another name. Use this index to locate the synonym for the site name used throughout the Index.

\section{Section 8: Index of Sites by Conventional Radiocarbon Age (TL and OSL dates excluded)}

This index lists all the radiocarbon dates in descending order of conventional radiocarbon age. $\mathrm{TL}$ and OSL dates are not included $(\mathrm{n}=17)$. Note that no differentiation is made between radiocarbon dates obtained on terrestrial (e.g. charcoal) or marine (e.g. shell) samples. Many dates based on marine samples may require corrections of up to several hundred years to make them comparable to dates obtained on terrestrial samples. This index is useful for locating determinations and/or sites for specific time periods.

\section{Section 9: Index of Sites by Region}

This index presents a list of dated sites grouped by region. A region is an arbitrary geographical referent devised for the purposed of the Index, based on a division of Queensland into six broad zones (see Figure 3). See the Explanatory Notes below for a full description of field data.

\section{Section 10: Index of Sites by 1:250,000 Map Sheet Name}

This index, which presents a list of dated sites grouped by $1: 250,000$ map sheet name, is particularly useful if one wants to locate all the dated sites in a specific area. The general area that each map sheet covers is presented on the map sheet key diagram (Figure 3).

\section{Section 11: References Cited}

This section provides full bibliographic information for every source cited in the Index.

\section{A Note on Conventional Radiocarbon Ages}

Radiocarbon ages in the Index are reported as conventional radiocarbon ages (Stuiver and Polach 1977). That is, they are corrected for isotopic fractionation and not corrected for marine reservoir effect or any other correction (cf. Kelly 1982; Thom et al. 1981). Three dates from the Broadbeach Burial Ground and one from Polka Point were originally reported by Bermingham (1971) without correction for fractionation. To convert these dates to conventional radiocarbon ages, a correction estimate for isotopic fractionation (derived from Stuiver and Reimer 1993) was applied and is included in the determinations reported for these sites in Section 1. See the Explanatory Notes below for a technical definition of the conventional radiocarbon age.

\section{A Note on Calibration of Radiocarbon Dates}

Radiocarbon ages are conventionally reported in ${ }^{14} \mathrm{C}$ years rather than calendar years. The primary difference between the two time-scales is caused by variability in the proportion of radioactive carbon in the biosphere through time and space. Conversion (or calibration) of radiocarbon dates from ${ }^{14} \mathrm{C}$ years to calendar years is possible by reference to records of known variability in radioactive carbon activity derived from dated tree-ring and coral-varve sequences. Such sequences enable direct comparisons to be made between radiocarbon dates and true calendar ages calculated from growth bands to derive a correction for a specific time period and region to convert ${ }^{14} \mathrm{C}$ years to calendar years. Technical details of the procedure used for calibrating radiocarbon dates for inclusion in the Index are presented in the Explanatory Notes (below). Calibration is a complex issue and for accessible discussions the reader is referred to Aitken (1990), Taylor (1987) and Taylor and Aitken (1997). 
Table 2. Summary of dates not available for inclusion in the Index.

\begin{tabular}{|c|c|c|c|c|}
\hline Site & \# of Dates & Method & Investigator & References \\
\hline Abbot Point & 10 & $14 \mathrm{C}$ & Bryce Barker & Barker pers. comm., 2000 \\
\hline Anvil Creek & 2 & $14 \mathrm{C}$ & Malcolm Ridges & Ridges et al. 2000 \\
\hline Border Island & 1 & $14 \mathrm{C}$ & Lara Lamb & Lamb pers. comm., 2000 \\
\hline Cuckadoo 3 & 1 & $14 \mathrm{C}$ & Malcolm Ridges & Ridges et al. 2000 \\
\hline Hay Cave & 11 & $14 \mathrm{C}$ & Harry Lourandos & Holden 1999; Roche 1999 \\
\hline Jiyer Cave & 5 & $14 \mathrm{C}$ & Richard Cosgrove & Cosgrove 2000 \\
\hline Kurkur Weid Rockshelter & 1 & $14 \mathrm{C}$ & Melissa Carter & Carter pers. comm., 2000 \\
\hline Lazaret Midden & 9 & $14 \mathrm{C}$ & Anne Ross & Ross 2000; Ross \& Coghill 1996 \\
\hline Moonface Site Rock Art & 1 & AMS & Malcolm Ridges & Ridges et al. 2000 \\
\hline Nara Inlet 1 & 1 & $14 \mathrm{C}$ & Lara Lamb & Lamb pers. comm., 2000 \\
\hline Ochre Quarry & 2 & AMS & Malcolm Ridges & Ridges et al. 2000 \\
\hline Ochre Quarry Shelter & 1 & $14 \mathrm{C}$ & Malcolm Ridges & Ridges et al. 2000 \\
\hline Ormi & 1 & $14 \mathrm{C}$ & Melissa Carter & Carter pers. comm., 2000 \\
\hline Pitkik 1 Midden & 1 & $14 \mathrm{C}$ & Melissa Carter & Carter pers. comm., 2000 \\
\hline Sokoli Beach & 1 & $14 \mathrm{C}$ & Melissa Carter & Carter pers. comm., 2000 \\
\hline Sokoli Beachrock & 1 & $14 \mathrm{C}$ & Melissa Carter & Carter pers. comm., 2000 \\
\hline Sokoli Midden & 4 & $14 \mathrm{C}$ & Melissa Carter & Carter pers. comm., 2000 \\
\hline Tigershark Rockshelter & 3 & $14 \mathrm{C}$ & Ian McNiven & McNiven pers. comm., 2000 \\
\hline
\end{tabular}

International lists and databases of dates often do not include calibrated (calendar) age-ranges for radiocarbon determinations (e.g. Breshini et al. 1996; Lavell et al. 1992; McFadgen et al. 2000). Two reasons are commonly cited for this omission. First, various calibration methods using the same calibration curve data can produce different results (see Aitchison et al. 1989). Second, on-going revisions and extensions of the calibration curve may render calibrations based on current data-sets obsolete. For the Index we decided to include calibration results where possible for all radiocarbon determinations. This was thought to be an important consideration for the utility of the Index as, without calibration, not only are radiocarbon dates obtained on charcoal and marine samples not comparable, but radiocarbon dates are not directly comparable to TL or OSL determinations. We attempted to reduce possible variation in calibration results by calculating results at the two sigma calibrated age-range and using a single calibration method (see Explanatory Notes below for technical details). Although on-going research is expected to result in revisions and extensions to the established calibration curves, these are unlikely to have a dramatic impact on the overall corpus of Queensland dates. This is primarily because almost $85 \%$ of radiocarbon dates in Queensland date to less than 7,000 BP, and thus fall on that part of established calibration curves which have been subject to only minor revisions since 1986 (see Stuiver et al. 1998). Extensions to calibration curves will have the largest impact on the 51 radiocarbon dates older than available curves.

\section{A Note on Coverage}

The Index of Dates from Archaeological Sites in Queensland includes all absolute dates determined on materials from archaeological sites in Queensland known to the authors up to November 2000. It includes dates from non-cultural deposits underlying (e.g. Morwood et al. 1995a) or overlying (e.g. David 1998) cultural deposits as long as the dated samples are derived from the archaeological site itself. For example, TL dates obtained by Morwood et al. (1995a) for sterile deposits underlying the Sandy Creek 2 cultural deposits are included while TL determinations obtained $150 \mathrm{~m}$ downslope of Sandy Creek 1 are excluded. Environmental dates which are 
not directly related to archaeological deposits are completely excluded. The Index includes only dated sites. Many archaeological sites have been excavated in Queensland without radiometric dates being obtained (e.g. Haglund-Calley and Quinnell 1973; McNiven 1990a; Tugby and Tugby 1965). Further, there are many thousands of archaeological sites known in Queensland which have not been excavated or dated.

Table 2 summarises dates which were unavailable for inclusion in the Index for various reasons. For example, several dates presented at conferences or seminars have not yet appeared in the literature (Cosgrove 2000; Ross 2000; Ross and Coghill 1996), some papers containing dates were also published too late for inclusion (Barham 2000; Ridges et al. 2000) and finally, several dates were not included at the specific request of the principal investigators of the sites in question (Holden 1999; Roche 1999).

\section{How to Use the Index}

The Index should, at all times, be used in conjunction with the Explanatory Notes (see below) which provide detailed information about field contents and potential problems or uncertainties in the data presented. We reiterate that the Index should be considered as a reference tool only and not a substitute for the original published accounts which place the dates within an archaeological context and discuss potential technical or interpretative issues. In short, this compilation will provide the most benefit to users when it is employed in conjunction with the cited published accounts from which they have been derived.

There are a number of ways to search the Index. At a very basic level, for a site known to the user, one should peruse Sections 1-3 for date information from that site or Sections 4-5 for site information. If the name of a dated site is known but it cannot be found in the Index it is possibly listed under another name. Use the 'Index of Sites by Alternative Site Name' (Section 7) to locate the synonym for the site name used in the Index. If you are interested in all the dated sites investigated by a particular researcher in Queensland you can use the 'Index of Sites by Principal Investigator' (Section 6) to find out which sites in the Index have been investigated by a particular person and then look at Sections 1-5 for further details. Alternatively, if you have no idea which sites may have been dated in a particular geographical area you can locate the area on the map sheet key diagram (Figure 3) and then use the 'Index of Sites by 1:250,000 Map Sheet Name' (Section 10) to find out which sites in the Index have been dated in a particular area and then refer to Sections 1-5 for further details.

\section{General Considerations}

Sites are arranged in alphabetical order of the most commonly cited name of the site. For convenience, dates for each site are arranged chronologically by conventional radiocarbon age (or TL or OSL determination), regardless of the sample material or square or whether the date is considered anomalous. Thus, for example, for some coastal sites where both marine shell and charcoal have been dated or where samples have been dated from different parts of the site this order will not necessarily reflect actual calendrical or stratigraphic ordering of the dates. Where two or more dates from a site gave exactly the same age they are arranged in ascending order of the standard deviation. Where both dates and standard deviations are the same from the same site they are arranged in alphanumeric order of the laboratory code.

\section{Future Updates}

This list is necessarily a work-in-progress. Inevitably there will be errors and omissions in this compilation. A version of all the data presented here should be available on the World Wide Web in the near future and further hard copy editions will be issued. The potential success of such complilations is evident in Breschini et al.'s (1996) California Radiocarbon Dates, which is now in its eighth edition and includes over 4,600 radiocarbon dates. Any corrections or additions to this list would be appreciated and should be directed to the authors.

\section{Acknowledgements}

The Index has largely been compiled in our spare time. We are indebted to the Aboriginal and Torres Strait Islander Studies Unit at the University of Queensland for the use of its facilities and resources in bringing the project to fruition.

We are grateful for the assistance and encouragement of many people who saw a value in this compilation when it seemed like yet another thing to do in their already busy lives. In particular, for advice and discussions on various aspects of the project thanks to Ms Brit Asmussen (School of Archaeology and Anthropology, Australian National University), Dr Tony Barham (Division of Archaeology and Natural History, Australian National University), Ms Deborah Brian (School of Social Science, University of Queensland), $\mathrm{Mr}$ Malcolm Connolly (Cultural Heritage Branch, Queensland Environmental Protection Agency), A/Prof. Jay Hall (Archaeology Program, School of Social Science, University of Queensland), Dr Alan Hogg (Waikato Radiocarbon Laboratory, University of Waikato), A/Prof. Ian Lilley (Aboriginal and Torres Strait Islander Studies Unit, University of 
Queensland), Mr Rob Neal (Queensland Environmental Protection Agency), Dr Jon Prangnell (University of Queensland Archaeological Services Unit), Dr Paula Reimer (School of Archaeology and Palaeoecology, Queen's University of Belfast), Dr Richard Robins (Australian Archaeology Section, Queensland Museum), Dr Annie Ross (Archaeology Program, School of Social Science, University of Queensland), Mr Mike Rowland (Cultural Heritage Branch, Queensland Environmental Protection Agency), Mr Jim Smith (Archaeology Program, School of Social Science, University of Queensland), Dr Graeme Ward (Australian Institute of Aboriginal and Torres Strait Islander Studies) and Ms Catherine Westcott (Cultural Heritage Branch, Queensland Environmental Protection Agency).

For responding to requests for additional information, checking entries and supplying unpublished material we sincerely thank: Dr Bryce Barker (Department of Humanities and International Studies, University of Southern Queensland), Dr John Beaton (Department of Anthropology, University of California, Davis), Mr Andrew Border (Queensland Environmental Protection Agency), Dr Noelene Cole, Dr Richard Cosgrove (Department of Archaeology, La Trobe University), Dr Bruno David (School of Geography and Environmental Science, Monash University), A/Prof. Jay Hall, Ms Lara Lamb (School of Archaeology and Anthropology, Australian National University), Dr Ian McNiven (School of Fine Arts, Classical Studies and Archaeology, University of Melbourne), Mr Rob Neal, Ms Norma Richardson (School of Archaeology and Anthropology, Australian National University), Dr Richard Robins and Mr Mike Rowland.

In particular, we would like to thank Mr Tony Eales, A/Prof. Jay Hall, A/Prof. Ian Lilley, Dr Ian McNiven, Dr Richard Robins and Mr Mike Rowland for making original radiocarbon laboratory reports available for direct cross-checking.

We are indebted to Dr Jon Prangnell for helping in the arduous task of plotting sites from often poorquality maps and to $\mathrm{Mr}$ Malcolm Connolly for helping with database advice and providing state site designations at the eleventh hour.

Finally, we would like to extend a special thanks to Mr Mike Rowland for encouragement throughout the project and for reading and commenting on the completed manuscript. We remain responsible for any errors or omissions. 


\section{Explanatory Notes}

\section{SECTION 1: Basic Determination Data}

Site Name: The most commonly-cited name of the site from which dated samples are derived. Alternative published designations are included in the 'Index of Sites by Alternative Site Name' (Section 7). Dates obtained from rock art are distinguished from dates obtained from sedimentary archaeological deposits by adding the suffix 'Rock Art' after the site name. This is a mandatory field for inclusion in the Index.

Lab. Code: A unique laboratory code assigned to an individual radiometric age determination. Table 3 provides a key to laboratory code abbreviations. Occasionally, determinations may have more than one code, particularly when sample preparation is conducted at one laboratory and actual age determination at another. Alternative laboratory codes are recorded in one of the 'Notes' fields in Section 3 (see below). Field or laboratory designations given to samples by submitters are excluded completely. Three determinations from three sites ( $\mathrm{St}$ Bees Rockshelter, Selwyn Range 1 and Selwyn Range 2) do not have laboratory codes reported in the literature. Shaded laboratory codes indicate that notes concerning aspects of the determination are listed in Section 3.

Method: The specific method used to arrive at an absolute age value for the sample. Four methods have been employed in absolute radiometric dating of archaeological deposits in Queensland: conventional radiocarbon $\left({ }^{14} \mathrm{C}\right)$, accelerator mass spectrometry (AMS), thermoluminesence (TL) and optically-stimulated luminescence (OSL). Please note that the Index is overwhelmingly focussed on radiocarbon (ie. ${ }^{14} \mathrm{C}$ and AMS) determinations so many data fields are not relevant to TL or OSL determinations. Where a particular field is not relevant to a TL or OSL determination 'NA' (Not Applicable) is inserted. Dates arrived at by non-absolute dating methods, including typological dating, electron spin resonance (ESR) dating (e.g. Caddie et al. 1984) and interpolations from absolute determinations, are excluded completely. For accessible discussions of these methods the reader is referred to Aitken (1990), Taylor (1987) and Taylor and Aitken (1997). This is a mandatory field for inclusion in the Index.

CRA: The conventional radiocarbon age (CRA) is employed, as defined by Stuiver and Polach (1977) as:

(1) the use of the Libby half-life value of 5568 years (mean life 8033 years);

(2) the assumption of uniformity in ${ }^{14} \mathrm{C}$ activity throughout the biosphere in the past;

(3) the use of oxalic acid or a secondary standard as the modern standard;

(4) isotopic fractionation normalization of all sample activities to the base of $\mathrm{d}^{13} \mathrm{C}=-25.0$ per mille (relative to the ${ }^{13} \mathrm{C}:{ }^{12} \mathrm{C}$ ratio of PDB standard); and,

(5) the use of AD 1950 as the base year, with ages given in years before present (BP) (i.e. AD $1950=0 \mathrm{BP}$ ).

The conventional radiocarbon age BP is calculated using the radiocarbon decay equation:

\section{$\mathbf{t}=-\mathbf{8 0 3 3} \ln ($ Asn/Aon $)$}

where -8033 represents the mean lifetime of ${ }^{14} \mathrm{C}$ (Stuiver and Polach 1977), Aon is the activity in counts per minute of the modern standard, Asn is the equivalent counts per minute for the sample and 'ln' represents the natural logarithm (see Higham 2000 for a discussion).

Exceptions to these conventions occur for very old and very young samples. Samples with CRAs of less than 200 years are termed 'modern' and those with CRAs more recent than AD 1950 (i.e. in the future) are termed '>modern' (greater than modern) (Stuiver and Polach 1977). In the Index both these designations are replaced by $0 \pm 0$. Very old samples, where ${ }^{14} \mathrm{C}$ activity cannot be confidently distinguished from the background laboratory ${ }^{14} \mathrm{C}$ activity, are reported as minimum ages without an estimate of standard error. For example, the result from Ngarrabullgan Cave in Cape York Peninsula is reported as >37,170 (Beta-45906) (David 1992a).

Note that this CRA definition is not applicable to TL or OSL determinations. The exact relationship between

${ }^{14} \mathrm{C}$ years, calendar years, TL years and OSL years is uncertain. This definition is different from the one employed by Kelly (1982), which followed Thom et al. (1981) in defining the CRA as corrected for environmental (marine) reservoir effects. This is a mandatory field for inclusion in the Index. 
士: The estimated standard error attached to an individual determination, equal to one standard deviation $(1 \sigma)$. Note that occasionally determinations have asymmetrical standard deviations.

Calibrated Age/s: Conventional radiocarbon ages (CRAs) were converted to calendar years using the CALIB (v4.3) computer program (Stuiver and Reimer 1993). Determinations based on charcoal and other terrestriallyderived samples (including land snail shell and freshwater shell) were calibrated using the atmospheric decadal dataset of Stuiver et al. (1998) with no laboratory error multiplier. Twenty-four years were subtracted from terrestrially-derived (atmospheric) samples before calibration to correct for ${ }^{14} \mathrm{C}$ variations between northern and southern hemispheres. Atmospheric CRA determinations between 0 BP and 20,265 BP were calibrated using the atmospheric decadal dataset (Stuiver et al. 1998). Dates on marine samples (e.g. marine and estuarine shell) with CRAs between 461 and c.20,760 BP were calibrated using the marine calibration model dataset of Stuiver et al. (1998) with a $\Delta \mathrm{R}$ correction value of $11 \pm 5$ (see Reimer 2000 for an outline of $\Delta \mathrm{R}$ values and calculation procedure). While the general validity of this $\Delta \mathrm{R}$ correction value for open waters has been sustained by recent research, significant variability in individual embayments and estuaries along the Queensland coast has been identified (Ulm in prep.). In the case of determinations based on marine and estuarine shell samples, the species and provenience of the sample should be considered on a case-by-case basis to assess the validity of the calibrated age presented. The calibrated ages reported span the $2 \sigma$ calibrated age-range. Samples too young for use of the calibration curves are reported as 'modern'. Where a calibrated intercept or age-range is reported as ' 0 *' a 'negative' or 'modern' age BP is indicated due to uncertainties introduced by nuclear testing. Atmospheric samples older than 20,265 BP and marine samples older than 20,760 BP are not able to be calibrated with any confidence using available calibration curves. Where a determination is older than available calibration curves, 'Beyond available calibration curves' is inserted. The dates presented on either side of the bracketed dates represent the $2 \sigma$ calibrated age-range of the radiocarbon date using the calibration procedure outlined above. The date/s in the brackets represent the intercept/s of the radiocarbon age with the calibration curve. In regions of the calibration curve exhibiting short-term variation in atmospheric radioactive carbon activity, or where radiocarbon ages have large standard errors, it is common to have multiple intercepts (i.e. multiple calibrated ages for any given radiocarbon date) which are equally probable.

Sample: The type of material upon which the age determination was obtained (e.g. charcoal, marine shell, freshwater shell, land snail shell, soil, wood, carbonate, bone, sediment etc). If the sample was speciated, this is recorded in a separate 'Species' field in Section 2 (see below). Where no sample type is provided in the literature the default value is 'Unidentified'.

Date Reference: This records the primary source of the reported determination. Wherever possible this is the earliest published record (including Honours theses) of the reported determination in its current form. Where determinations have been recalculated by laboratories or where dates have been misreported in earlier publications, the earliest corrected source of the determination is cited wherever possible. For the purposes of this field, postgraduate research theses of honours level and above are included. Wherever possible, unpublished material and personal communications are excluded where a published source is available. This is a mandatory field for inclusion in the Index.

\section{SECTION 2: Advanced Determination Data}

Site Name: The most commonly-cited name of the site from which dated samples are derived. Alternative published designations are included in the 'Index of Sites by Alternative Site Name' (Section 7). Dates obtained from rock art are distinguished from dates obtained from sedimentary archaeological deposits by adding the suffix 'Rock Art' after the site name. This is a mandatory field for inclusion in the Index.

Lab. Code: A unique laboratory code assigned to an individual radiometric age determination. Table 3 provides a key to laboratory code abbreviations. Occasionally, determinations may have more than one code, particularly when sample preparation is conducted at one laboratory and actual age determination at another. Alternative laboratory codes are recorded in one of the 'Notes' fields in Section 3 (see below). Field or laboratory designations given to samples by submitters are excluded completely. Three determinations from three sites ( $\mathrm{St}$ Bees Rockshelter, Selwyn Range 1 and Selwyn Range 2) do not have laboratory codes reported in the literature. Shaded laboratory codes indicate that notes concerning aspects of the determination are listed in Section 3. 
Species: The species of the material upon which the age determination was obtained (e.g. Anadara trapezia etc). This is commonly reported for marine shell and estuarine shell samples but less commonly determined for charcoal and other samples. Where no species is stated in the literature the default value is 'Unidentified'. Note that this field is not relevant for TL or OSL determinations where 'NA' (Not Applicable) is inserted.

Weight: The weight of the sample submitted in grams (g). Note that after physical and chemical laboratory pretreatment procedures the actual weight of material dated can be considerably less than that submitted. Where no weight is stated in the literature, the field is left blank.

Square: The field-designated horizontal unit of excavation from which the sample was derived. In recent excavations this unit is normally a square forming part of a metric excavation grid. It also includes other spatial designations such as 'trench'. Where no square or other spatial designation is stated in the literature, the field is left blank.

XU: The excavation unit (XU) is the field-designated vertical unit of excavation from which the sample is derived. In recent excavations this unit has commonly been an arbitrary depth unit within a horizontal unit (see 'Square' above) or an arbitrary depth unit within a stratigraphic unit. Where only stratigraphic units (or layers) are available these are included with the prefix 'SU'. Where no excavation unit or equivalent is stated in the literature, the field is left blank.

Depth: The depth of the sample below ground surface, in centimetres $(\mathrm{cm})$. This is normally presented in the form of a depth range, generally according to the beginning and end depths of the excavation unit (see 'XU' above) from which the sample is derived. Occasionally an absolute depth value is presented, normally resulting from plotting of the sample during excavation. Where no depth is stated in the literature, the field is left blank.

d14C: The depletion of ${ }^{14} \mathrm{C}$ in a sample before correction for fractionation, expressed per mille (\%) (Aitken 1990; Higham 2000). d14C is calculated by (see 'CRA' above for definitions):

\section{d14C $=(($ Asn/Aon $)-1) 1000$ per mille}

土: Standard deviation of the determined d $14 \mathrm{C}$ value (see above), normally equal to one standard deviation ( $1 \sigma$ ).

d13C: The difference between the ${ }^{13} \mathrm{C}:{ }^{12} \mathrm{C}$ ratio of the sample to the ratio of the chosen standard (Aitken 1990:63), expressed per mille (\%o). Conventionally, the standard employed is PDB carbonate (Belemnita americana, from the Pee Dee formation of South Carolina) (Aitken 1990:110). Note that where this value has been estimated, rather than actually determined for the sample, an ' $\mathrm{e}$ ' is added at the end of the value.

$\pm:$ Standard deviation of the determined $\mathrm{d} 13 \mathrm{C}$ value (see above), normally equal to one standard deviation $(1 \sigma)$.

D14C: The depletion of ${ }^{14} \mathrm{C}$ in a sample after correction for isotopic fractionation of the sample to the base value of $-25.0 \%$ per mille (relative to the ${ }^{13} \mathrm{C}:{ }^{12} \mathrm{C}$ ratio of PDB standard), expressed per mille (\%o) (Aitken 1990). $\mathrm{D} 14 \mathrm{C}$ is given by:

\section{$D 14 C=d 14 C-2(d C 13+25)(1+d 14 C / 1000)$ per mille}

$\pm:$ Standard deviation of the determined D14C value (see above), normally equal to one standard deviation $(1 \sigma)$.

\%M: Percent modern (\%M or pMC - 'percent modern carbon') is calculated using:

$\% \mathrm{M}=100 \times$ x Asn/Aabs OR Asn/Aon(1/8267(y-1950)) x 100 percent

where Aabs is the absolute international standard activity, 1/8267 is the lifetime based on the new half life (5730 years), and y is the year of measurement of the appropriate standard. This is an expression of the ratio of the net modern activity against the residual normalised activity of the sample, expressed as a percentage. It represents the proportion of radiocarbon atoms in the sample compared to that present in the year AD 1950. 
$\pm:$ Standard deviation of the determined \%M value (see above), normally equal to one standard deviation $(1 \sigma)$.

\section{SECTION 3: Notes on Individual Determinations}

Site Name: The most commonly-cited name of the site from which dated samples are derived. Alternative published designations are included in the 'Index of Sites by Alternative Site Name' (Section 7). Dates obtained from rock art are distinguished from dates obtained from sedimentary archaeological deposits by adding the suffix 'Rock Art' after the site name. This is a mandatory field for inclusion in the Index.

Lab. Code: A unique laboratory code assigned to an individual radiometric age determination. Table 3 provides a key to laboratory code abbreviations. Occasionally, determinations may have more than one code, particularly when sample preparation is conducted at one laboratory and actual age determination at another. Alternative laboratory codes are recorded in one of the 'Notes' fields (see below). Field or laboratory designations given to samples by submitters are excluded completely. Three determinations from three sites (St Bees Rockshelter, Selwyn Range 1 and Selwyn Range 2) do not have laboratory codes reported in the literature.

Notes 1-2: This field records notes on aspects of data which require comment or clarification. Determinations with accompanying notes are demarcated by shading of the laboratory code field in Sections 1-2. Notes include instances where individual determinations or laboratory numbers are previously mis-reported or where a date is considered to be anomalous by an investigator. Please note that not all determinations have accompanying notes.

\section{SECTION 4: Site Information}

Site Name: The most commonly-cited name of the site from which dated samples are derived. Alternative published designations are included in the 'Index of Sites by Alternative Site Name' (Section 7). This is a mandatory field for inclusion in the Index.

Region: The field records a general, arbitrary geographical referent for the site based on a division of Queensland into six zones (see Figure 3, 4 or 5): $\mathrm{TS}=$ Torres Strait; CYP $=$ Cape York Peninsula; $\mathrm{NWQ}=$ Northwest Queensland; $\mathrm{CQ}=$ Central Queensland; SWQ=Southwest Queensland; and SEQ=Southeast Queensland. Where possible, these general divisions conform to everyday usage of the terms. These units are completely arbitrary and are not meant to imply any analytical category. The divisions of each region conform to boundaries of the 1:250,000 map sheet series so that a nested hierarchy of geographical referents is established. The only exception to this last point is the Torres Strait 1:250,000 map sheet. Sites on this map sheet which are on the mainland are included in the Cape York Peninsula (CYP) region whereas sites on islands are included in the Torres Strait (TS) region.

1:250K Sheet: The Queensland 1:250,000 map sheet (by name) on which the site occurs (see Figure 3, 4 or 5). The 1:250,000 map sheet series was chosen as this is the smallest scale series which covers the whole of Queensland and is the series used by the Cultural Heritage Branch of the Queensland Environmental Protection Agency for site recording procedures (Johnston and Rowland 1987).

Latitude: The decimal latitude of the site. For most sites this value has been estimated from sketch maps and general site location information included in the literature. Few sites reported in the literature include precise locational data. Thus there is considerable uncertainty in the precision of this value. In any case, it may not be desirable to be overly precise owing to the possible use of compilations such as this one for nefarious activities. This value has therefore been rounded to four decimal places to obscure exact site locations. The reader should, therefore, generally treat this value as an approximation and is referred back to the original literature reporting the site details.

Longitude: The decimal longitude of the site. For most sites this value has been estimated from sketch maps and general site location information included in the literature. Few sites reported in the literature include precise locational data. Thus there is considerable uncertainty in the precision of this value. In any case, it may 
not be desirable to be overly precise owing to the possible use of compilations such as this one for nefarious activities. This value has therefore been rounded to four decimal places to obscure exact site locations. The reader should, therefore, generally treat this value as an approximation and is referred back to the original literature reporting the site details.

State \#: The unique site designation assigned by the Cultural Heritage Branch, Queensland Environmental Protection Agency. Please note that Cultural Heritage Branch site designations are not necessarily the same as those assigned by archaeological investigators. For example, multiple sites defined as separate by investigators may have the same Cultural Heritage Branch designation, and vice versa. Many of these values were obtained from a geographic information system (GIS) overlay of the sites presented in the Index over those held by the Environmental Protection Agency. Some of these designations may be incorrect owing to uncertainties in locational data. The data in this field will be checked for the next edition. Note that not all sites in the Index have a state designation.

Type: This field records whether the site is situated in an open (i.e. open air) context or rockshelter (including cave) context.

Location: This field records whether the site is coastal or inland. Coastal sites are arbitrarily defined as sites within approximately $1 \mathrm{~km}$ of the current high water mark (including rivers and creeks).

Investigator 1-3: The principal investigator of the site during the period when the reported determination/s were undertaken. Some sites have multiple investigators through time when sites have been excavated on more than one occasion. The principal investigator is defined as the individual who took primary responsibility for the excavation at the time the determination/s in question were undertaken. Note that these fields exclude principal investigators who may have excavated at a site but did not actually obtain any chronometric determinations while fulfilling the role of principal investigator.

\section{SECTION 5: Site References}

Site Name: The most commonly-cited name of the site from which dated samples are derived. Alternative published designations are included in the 'Index of Sites by Alternative Site Name' (Section 7). This is a mandatory field for inclusion in the Index.

General Reference: This section lists references (including the primary 'Date Reference', see above) that refer to aspects of the site for which dates are presented. It should be noted that these references do not necessarily contain the dates themselves, but rather often provide other relevant information such as site descriptions, excavations procedures and stratigraphic profiles.

\section{Abbreviations}

\begin{tabular}{|ll|ll|}
\hline AMS & accelerator mass spectrometry & NA $\quad$ not applicable \\
\hline CRA $\quad$ conventional radiocarbon age & ORE $\quad$ oceanic reservoir effect (or marine reservoir effect) \\
\hline e $\quad$ estimate & OSL optically-stimulated luminescence \\
\hline f/w & freshwater & TL $\quad$ thermoluminescence \\
\hline l/s & land snail & \\
\hline
\end{tabular}


Table 3. Key to laboratory code abbreviations used in the text. An asterisk (*) indicates that the laboratory is no longer active.

\begin{tabular}{|c|c|c|}
\hline Code & Method & Laboratory Details \\
\hline $\mathrm{AA}$ & AMS & NSF-Arizona AMS Facility, University of Arizona, Tucsan, USA \\
\hline ANU & $14 \mathrm{C}$ & Quaternary Dating Research Centre, Australian National University, Canberra, Australia \\
\hline ANUA & AMS & Department of Nuclear Physics, Australian National University, Canberra, Australia \\
\hline $\mathrm{ANU}_{\mathrm{OD}}$ & OSL & Australian National University Optical Date, Luminescence Dating Laboratory, Canberra \\
\hline ARL & AMS & Australian Radiometric Laboratory \\
\hline Beta & $14 \mathrm{C}$ & Beta Analytic Inc., Miami, USA \\
\hline ETH & AMS & Eidgenossische Technische Hochschule Honggerberg, Zurich, Switzerland \\
\hline $\mathrm{GaK}$ & $14 \mathrm{C}$ & Radiocarbon Laboratory, Gakushuin University, Tokyo, Japan \\
\hline GX & $14 \mathrm{C}$ & Geochron Laboratories, Cambridge, Massachusetts, USA \\
\hline I & $14 \mathrm{C}$ & Teledyne Brown Engineering Environmental Services, Westwood, New Jersey, USA \\
\hline $\mathrm{LJ}^{*}$ & $14 \mathrm{C}$ & Scripps (UCSD), La Jolla, USA \\
\hline NPL* & $14 \mathrm{C}$ & National Physical Laboratory, Middlesex, UK \\
\hline NZA & AMS & Rafter Radiocarbon Laboratory, Lower Hutt, New Zealand \\
\hline OxA & AMS & Oxford Radiocarbon Accelerator Unit, Oxford University, UK \\
\hline $\mathrm{OZ}$ & AMS & ANTARES AMS Centre, ANSTO, Lucas Heights, Australia \\
\hline $\mathrm{R}$ & $14 \mathrm{C}$ & Dipartmento di Fisica, Universitia "La Sapienza”, Rome, Italy \\
\hline SUA & $14 \mathrm{C}$ & The NWG Macintosh Centre for Quaternary Dating, University Sydney, Sydney, Australia \\
\hline $\mathrm{V}^{*}$ & $14 \mathrm{C}$ & Victoria Radiocarbon Laboratory, Melbourne, Australia \\
\hline $\mathrm{W}$ & TL & University of Wollongong Thermoluminescence Dating Laboratory, Wollongong, Australia \\
\hline Wk & $14 \mathrm{C}$ & Radiocarbon Laboratory, University of Waikato, Hamilton, New Zealand \\
\hline
\end{tabular}




\section{SECTION 1:}

\section{Basic Determination Data}




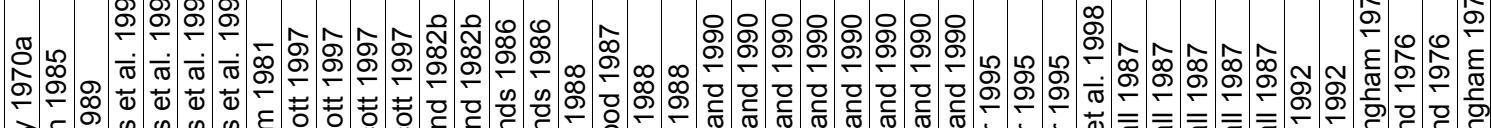

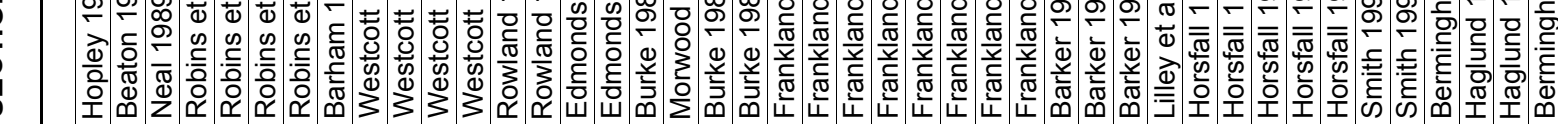

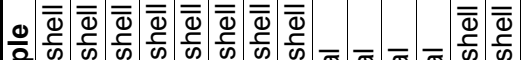

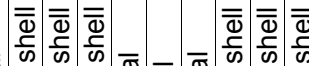

空

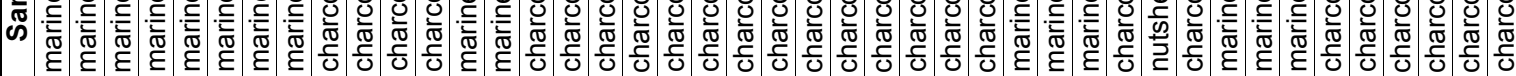

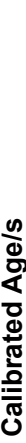

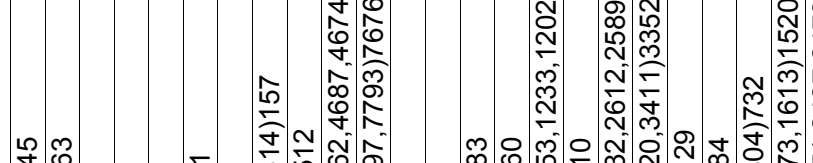

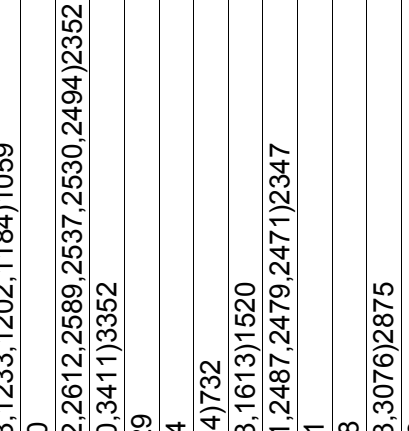

: \%

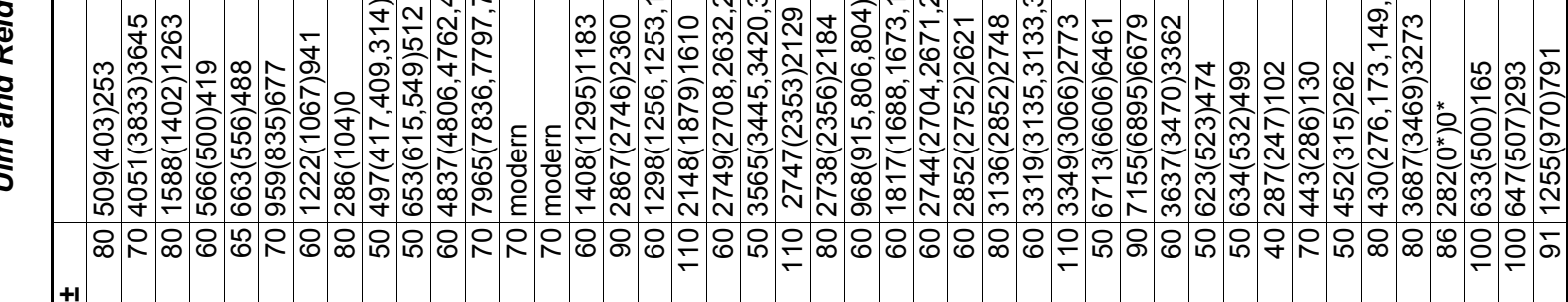

$+1$

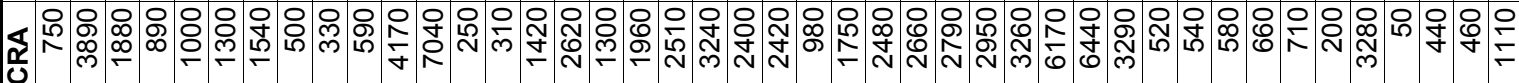

ס

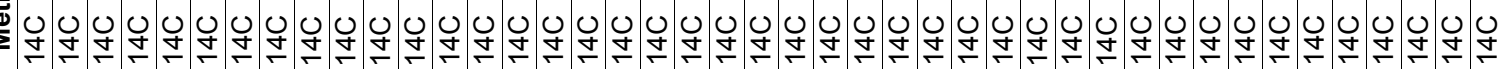

¿

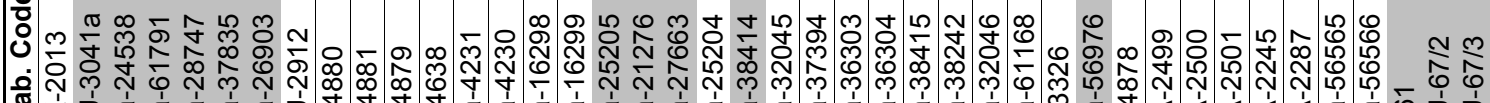

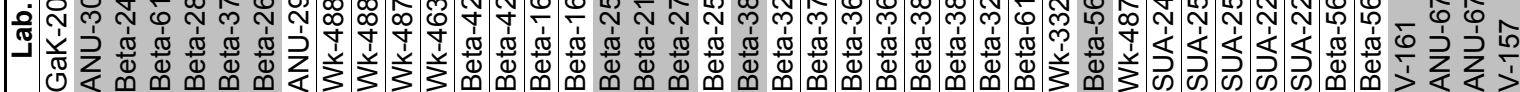

\begin{tabular}{|c|c|c|c|c|c|c|c|c|c|}
\hline 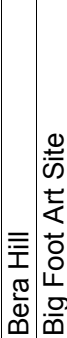 & 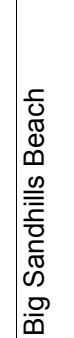 & 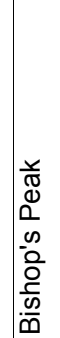 & 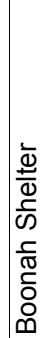 & 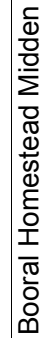 & 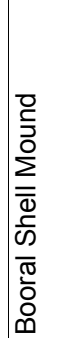 & 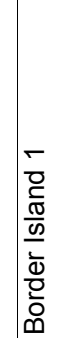 & 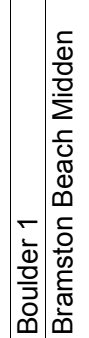 & 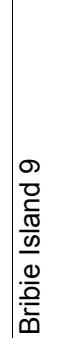 & 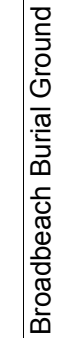 \\
\hline
\end{tabular}


(2)

次

冓

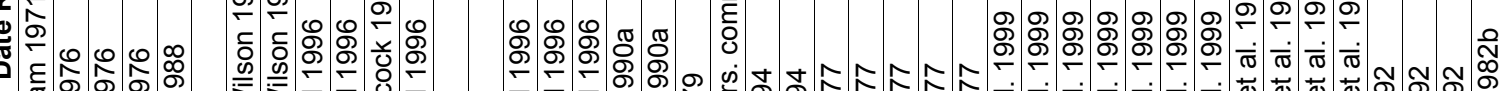

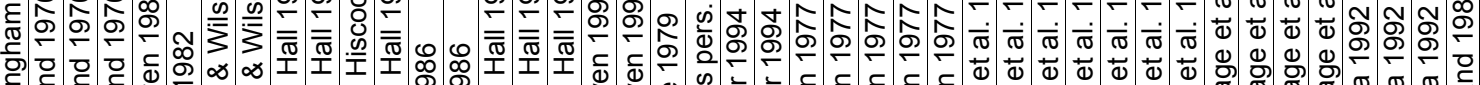

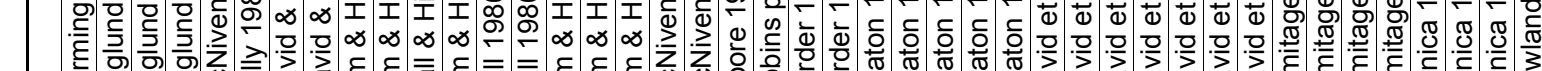

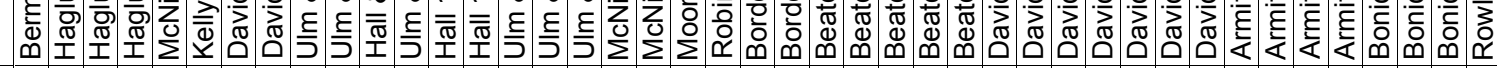

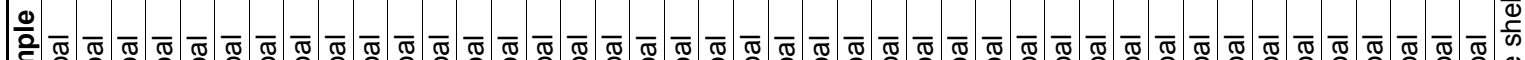

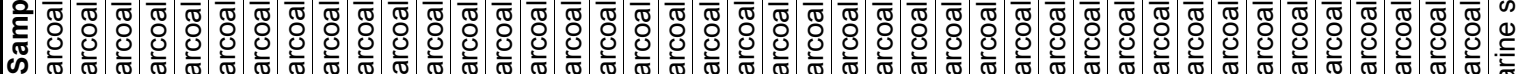

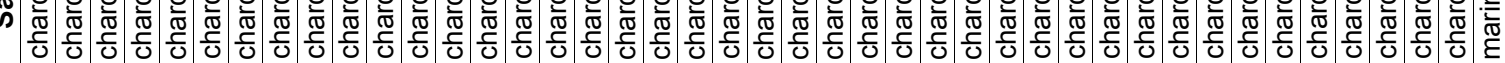

$\frac{n}{\mathscr{d}}$

$-\frac{1}{2}$

ऽั

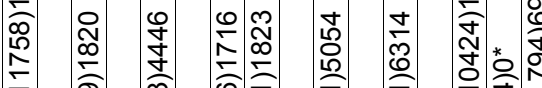

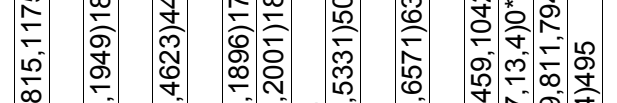

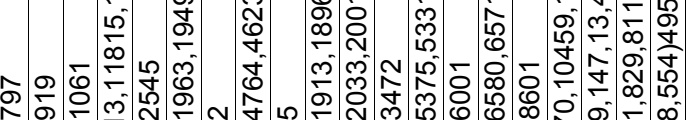

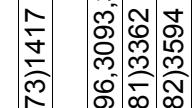

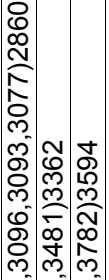

离

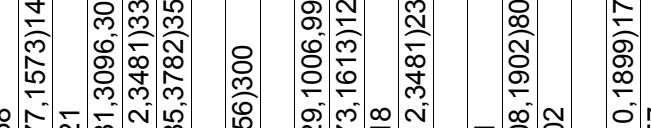

N

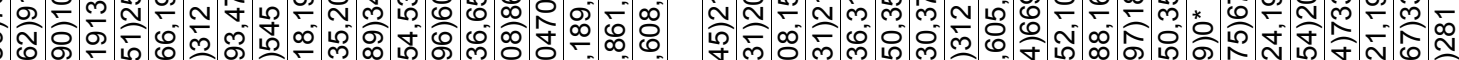

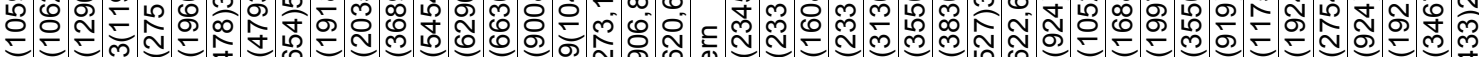

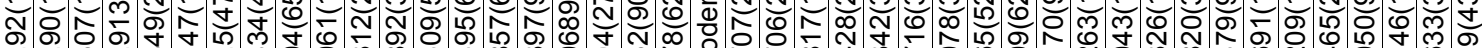

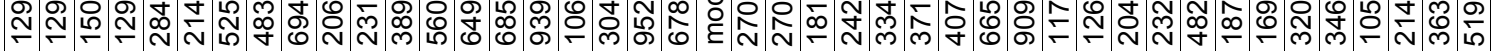

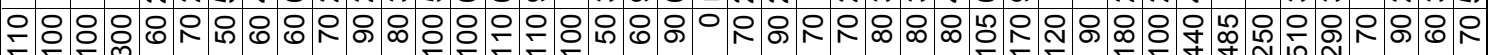
$+1$

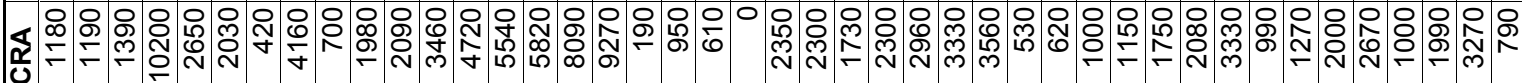

:

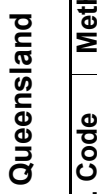

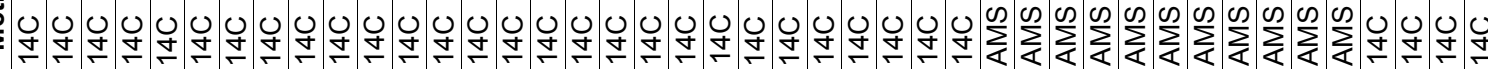

రํ.

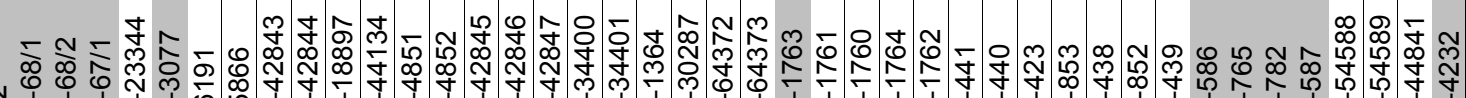

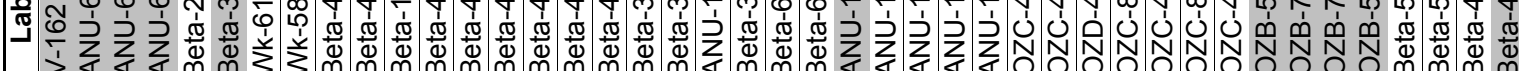

$\overline{\frac{8}{6}}$

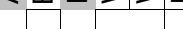
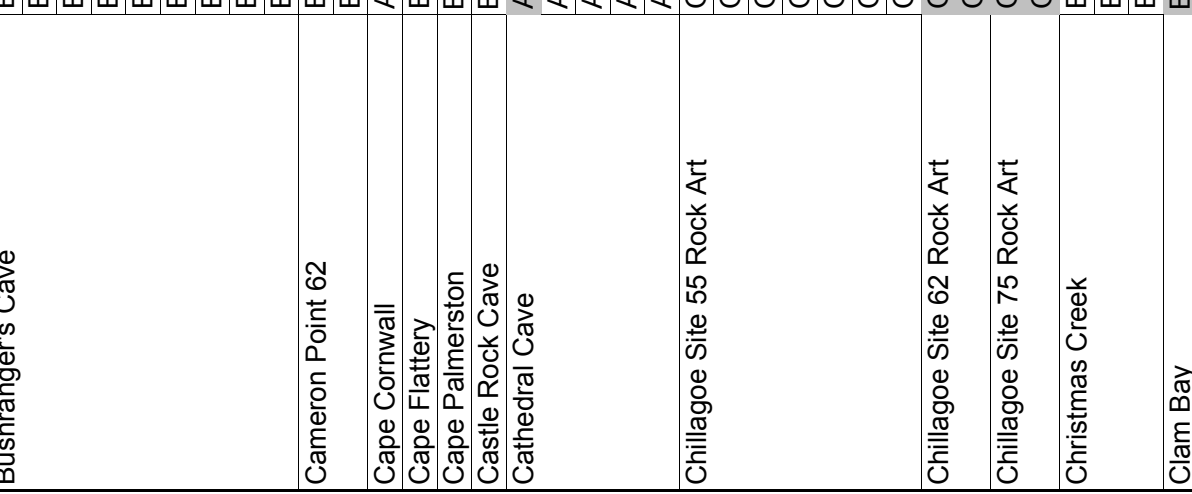

$\stackrel{2}{2}$

के

응 


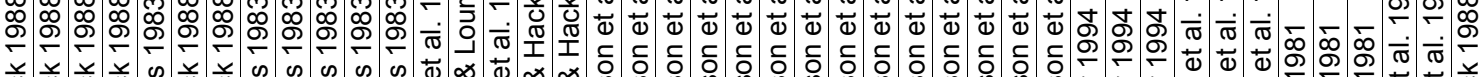

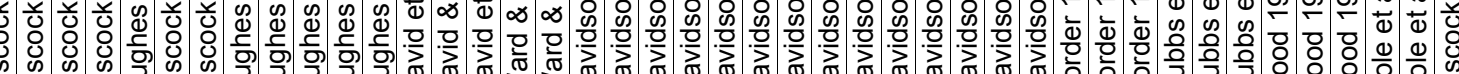

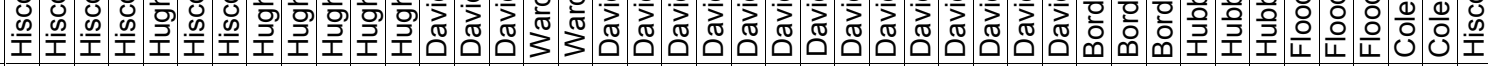

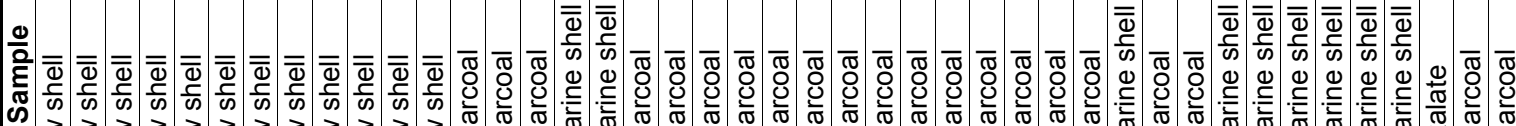

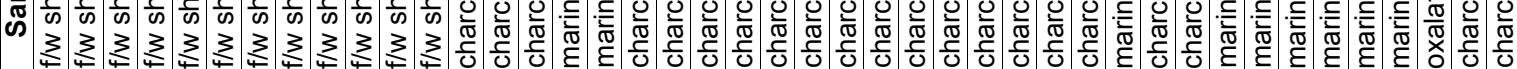

พิ

离离

$\stackrel{\sim}{\sim} \underset{\infty}{\sim}$

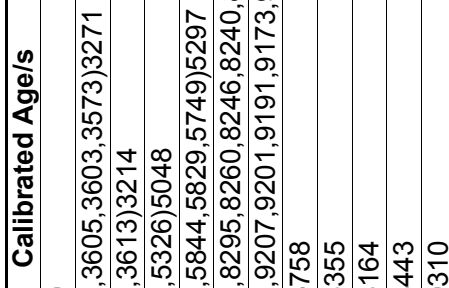

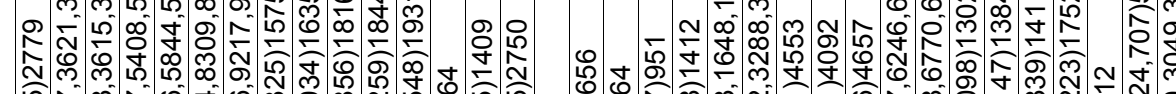

(2)

谊通

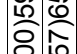

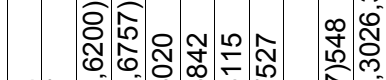

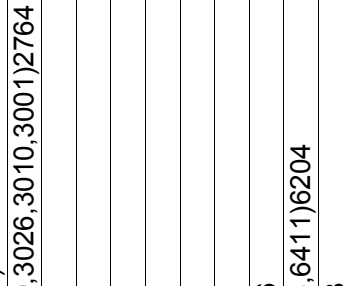

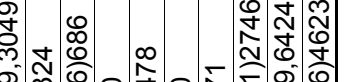

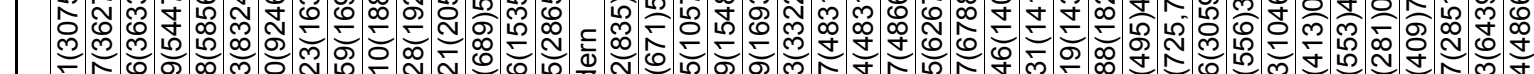
-

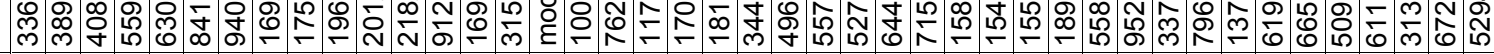

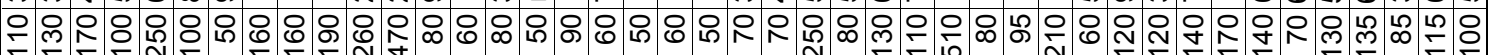

$+1$

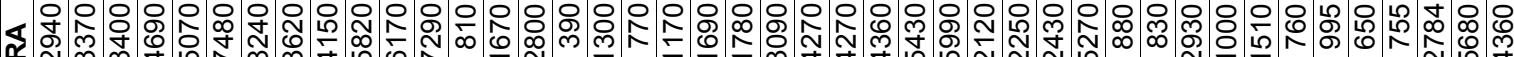
U.

¿

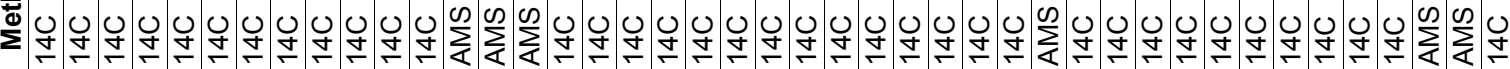

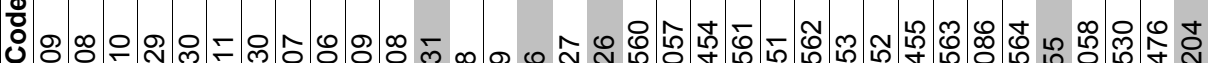

ن.

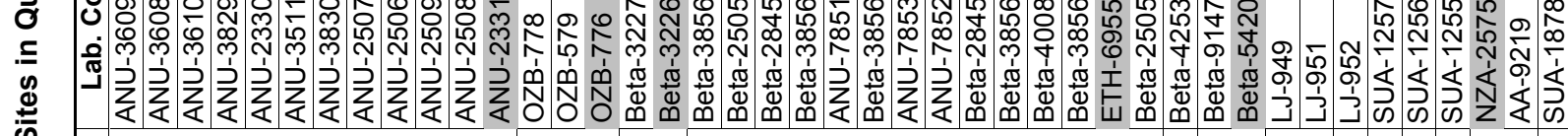

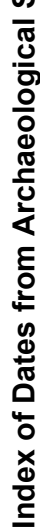

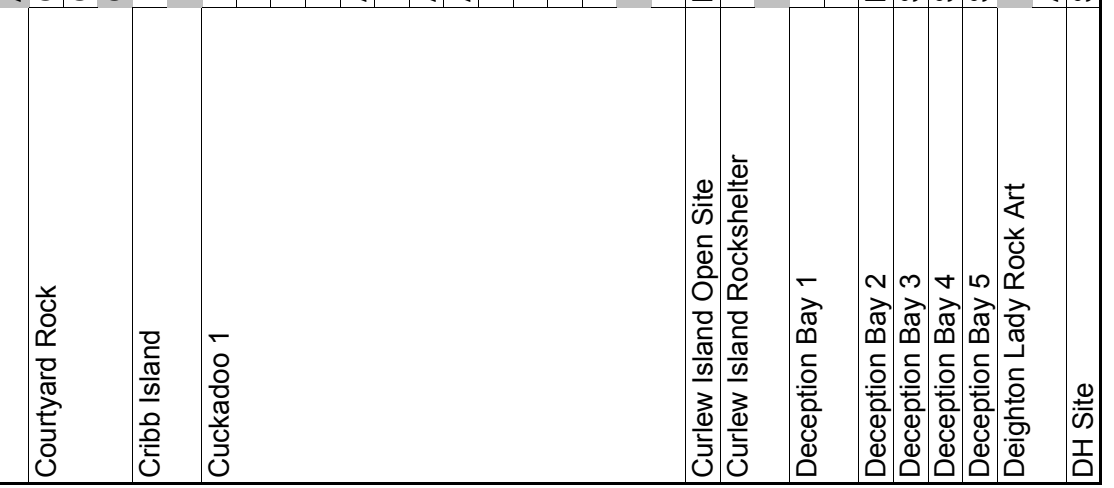

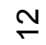

웅

8 


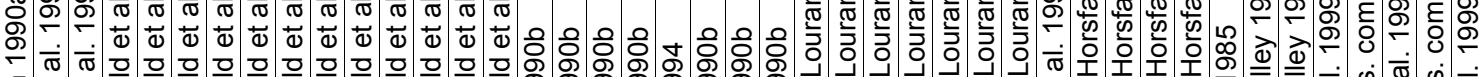

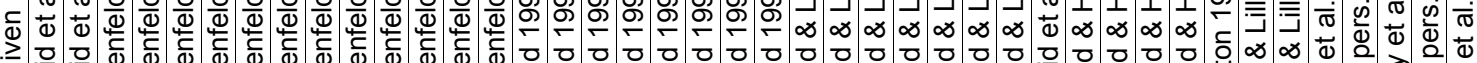

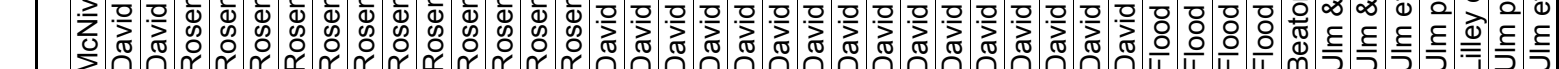

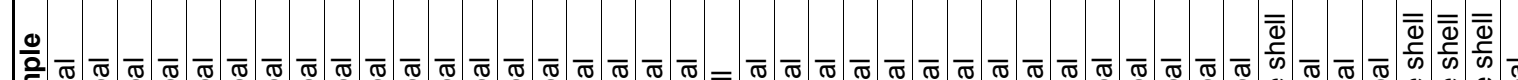

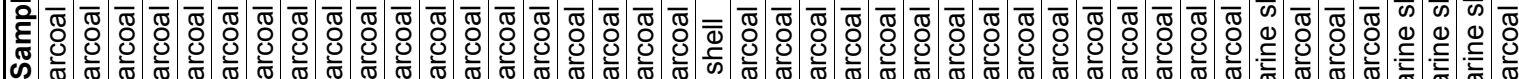

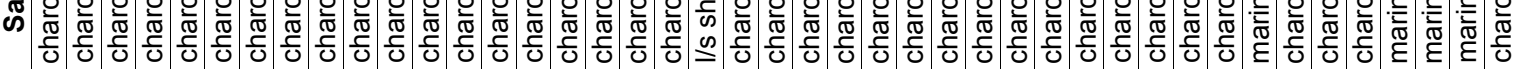

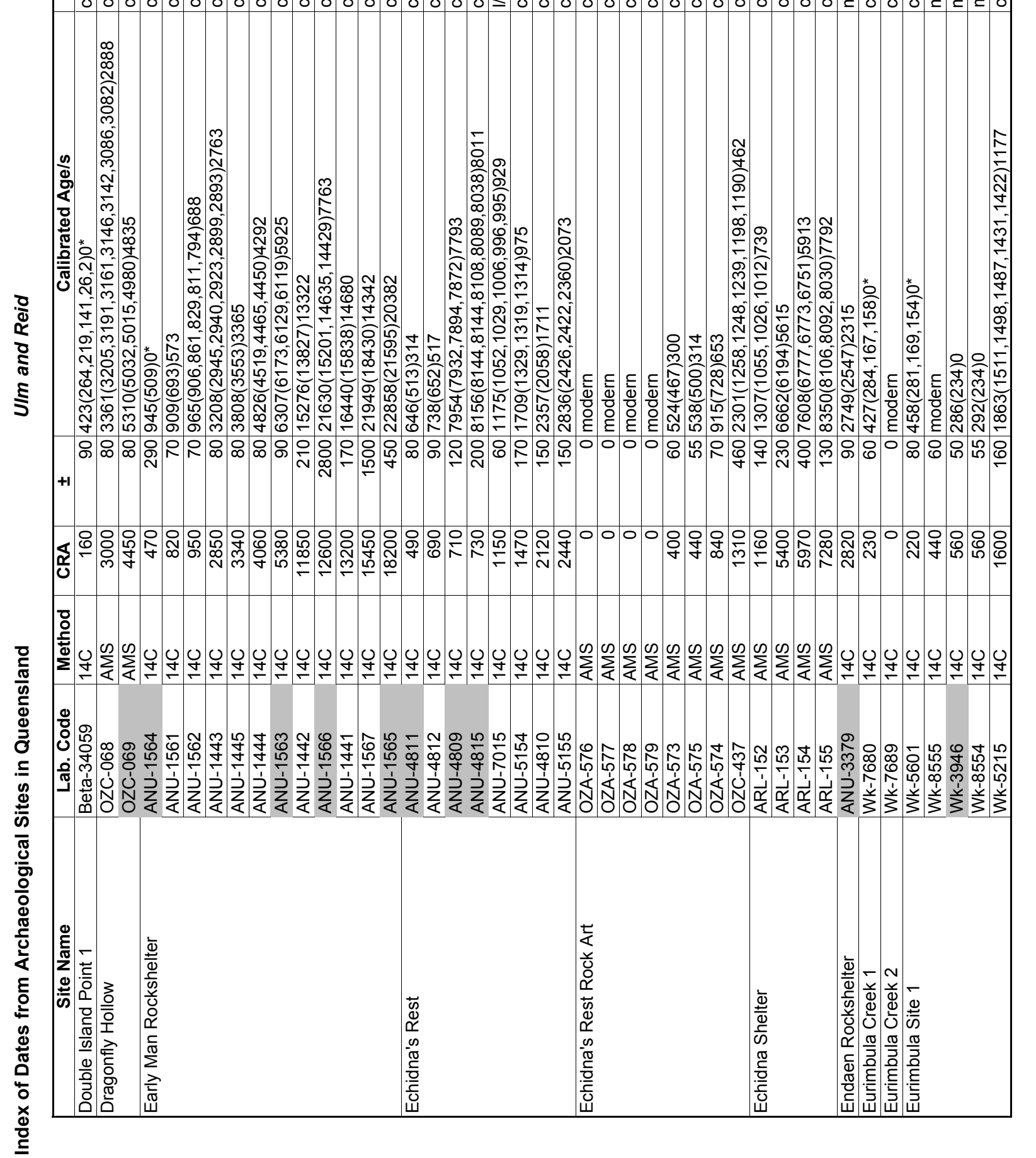


Q

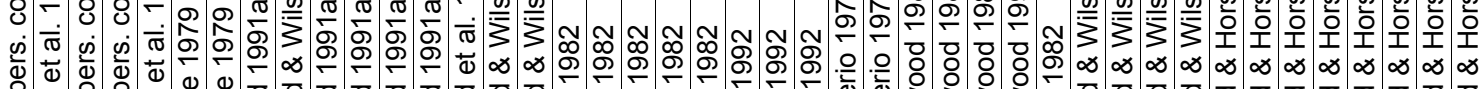

$\infty \infty \infty \infty \infty \infty$

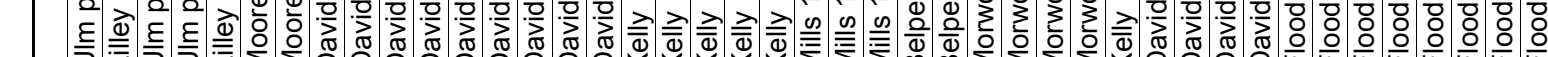

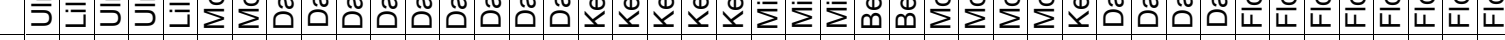

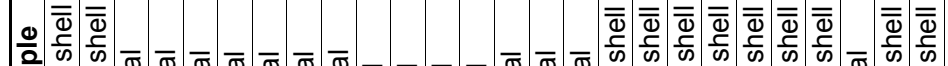

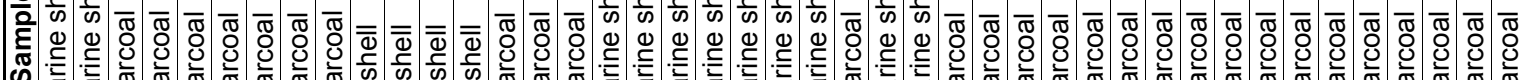
œ

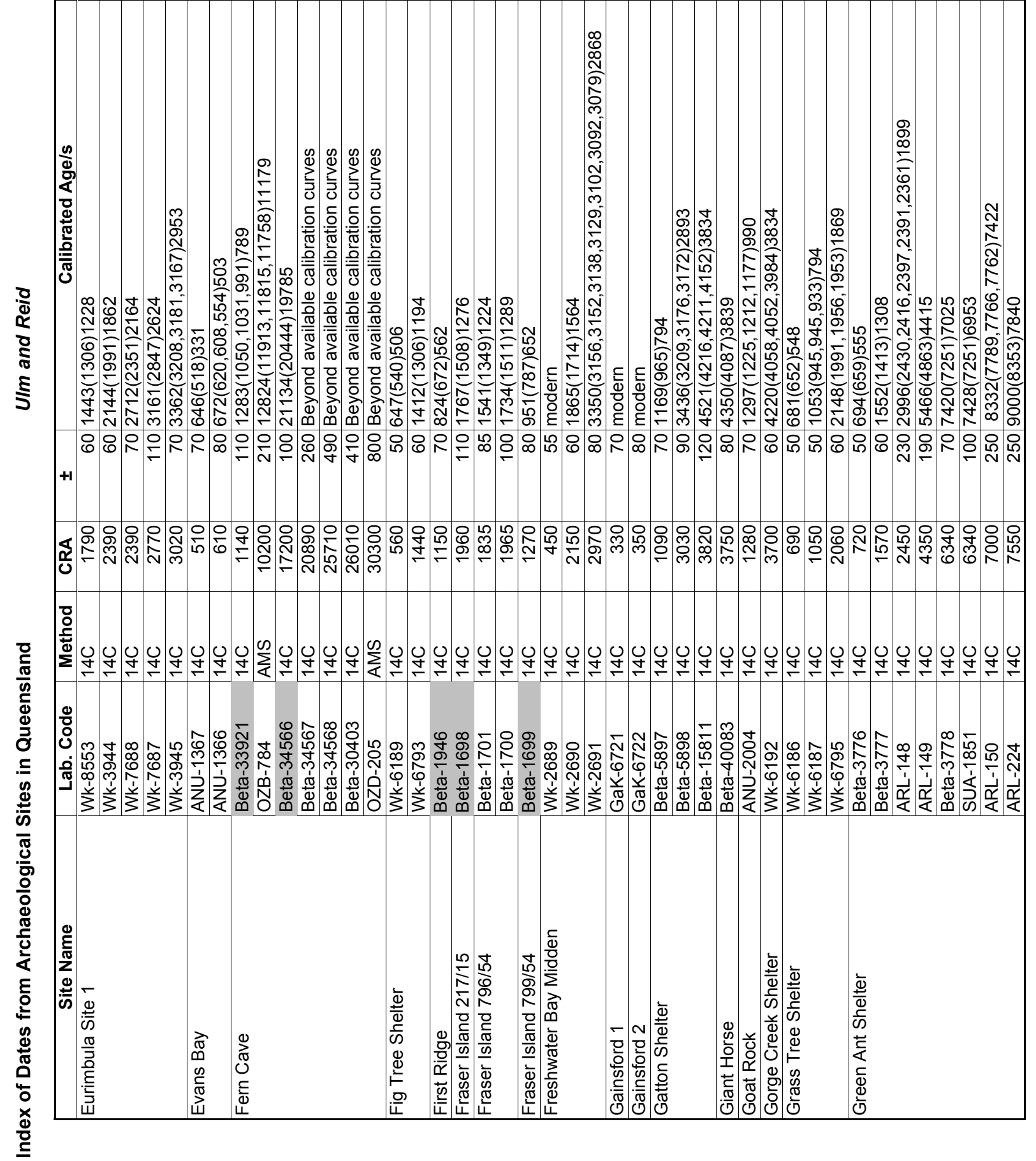


:

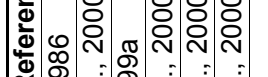

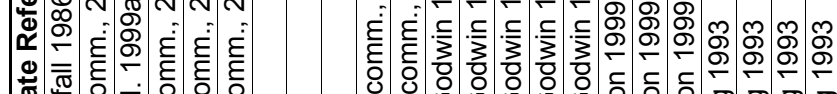

岕

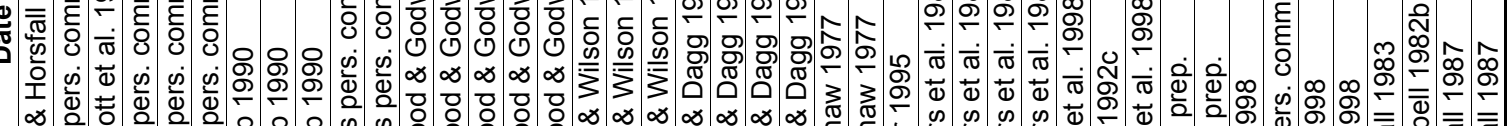

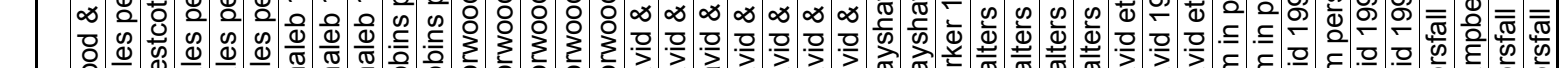

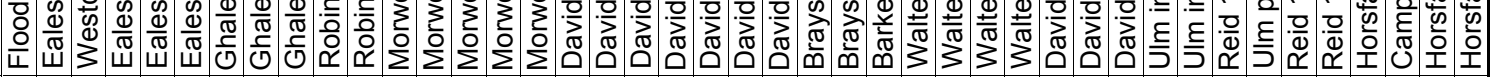

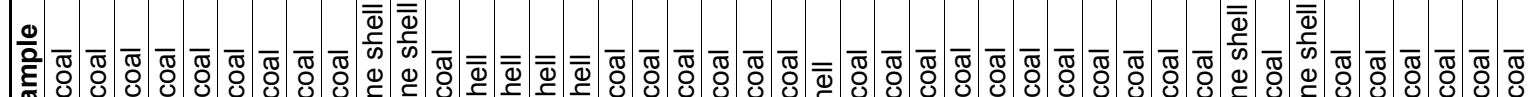

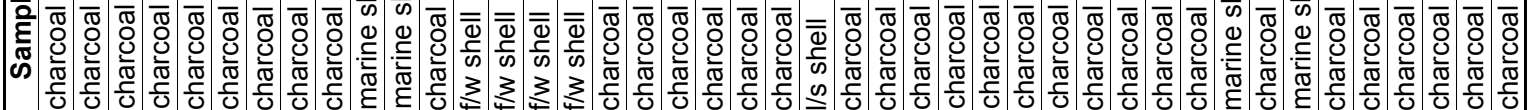

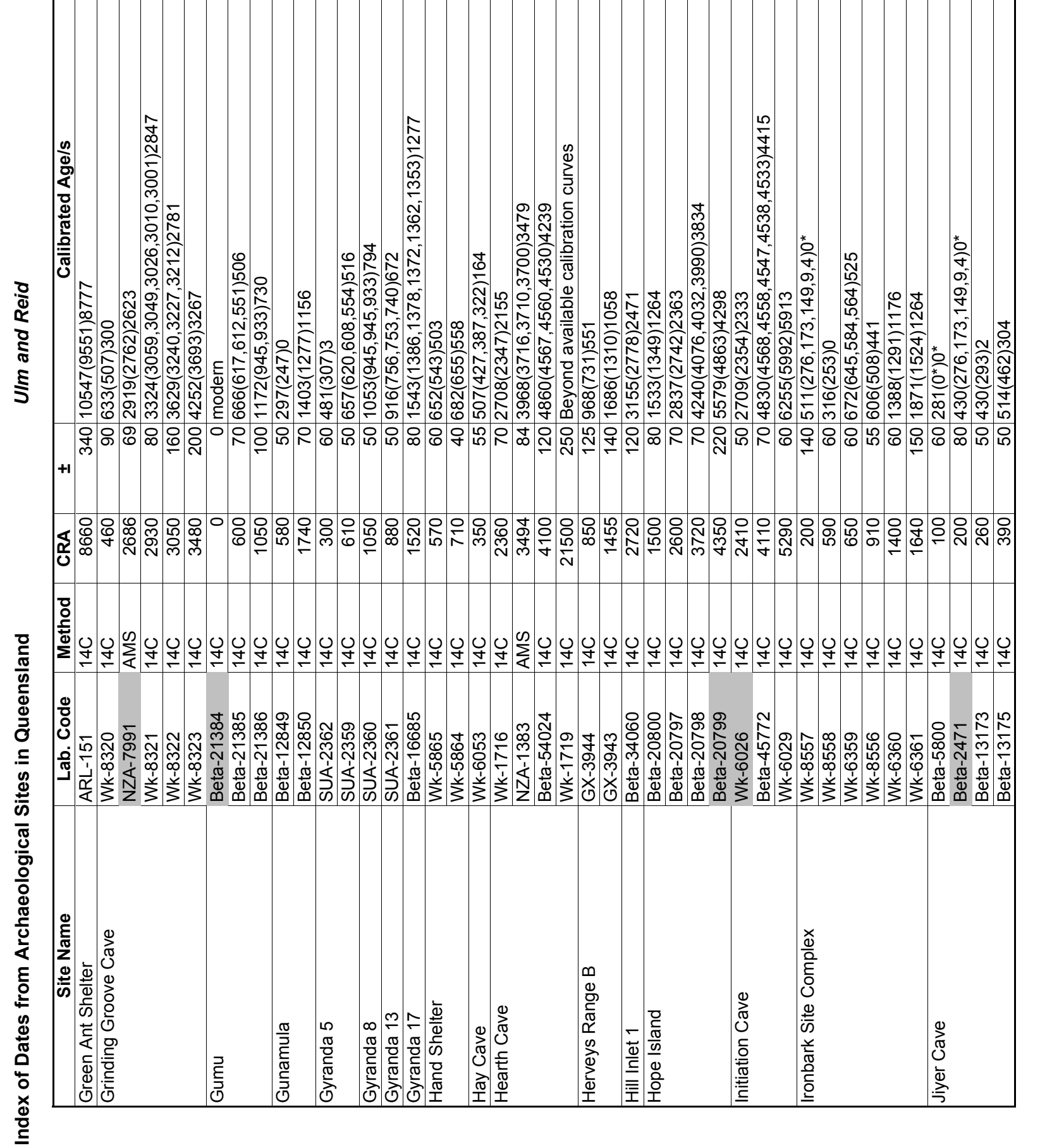

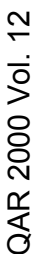




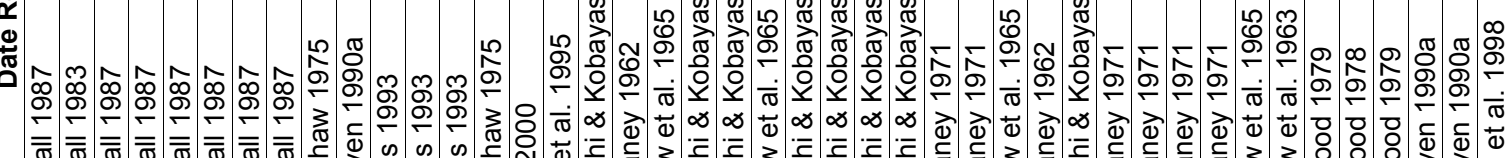

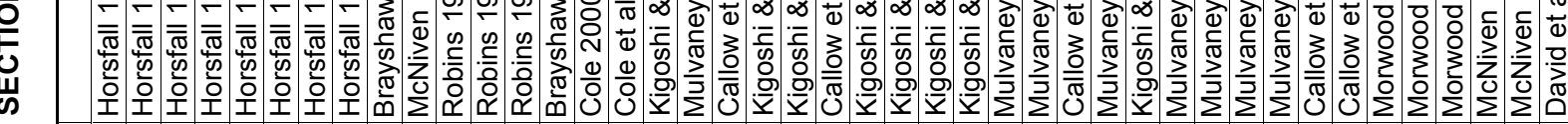

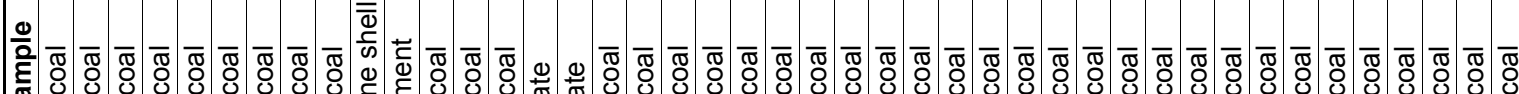

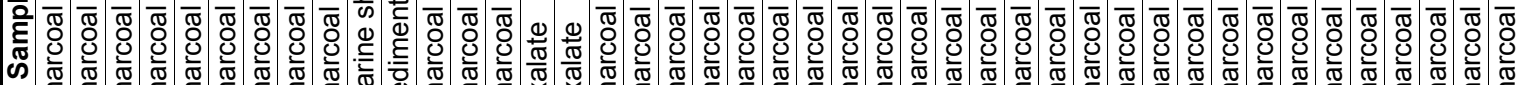
ڤ

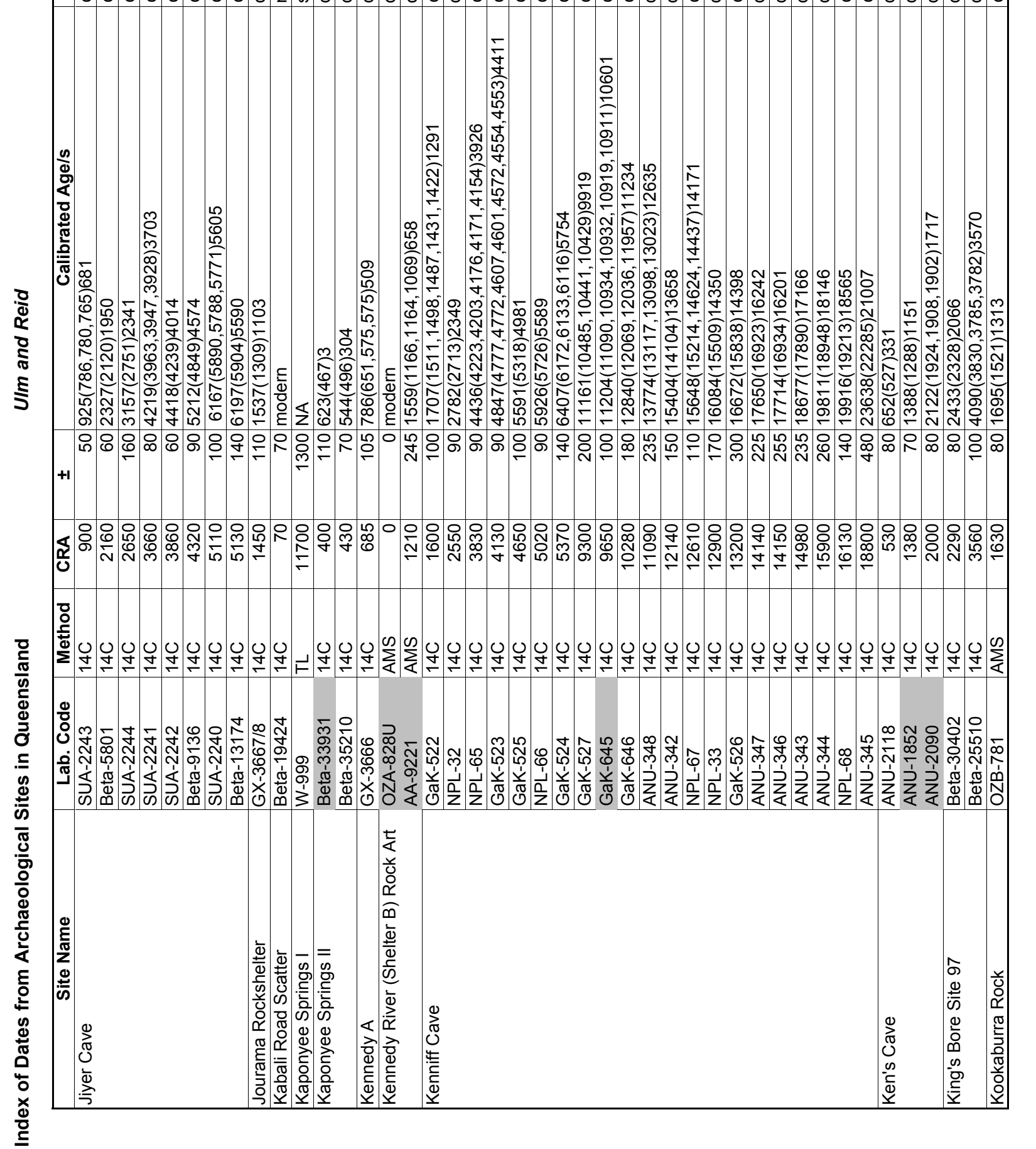




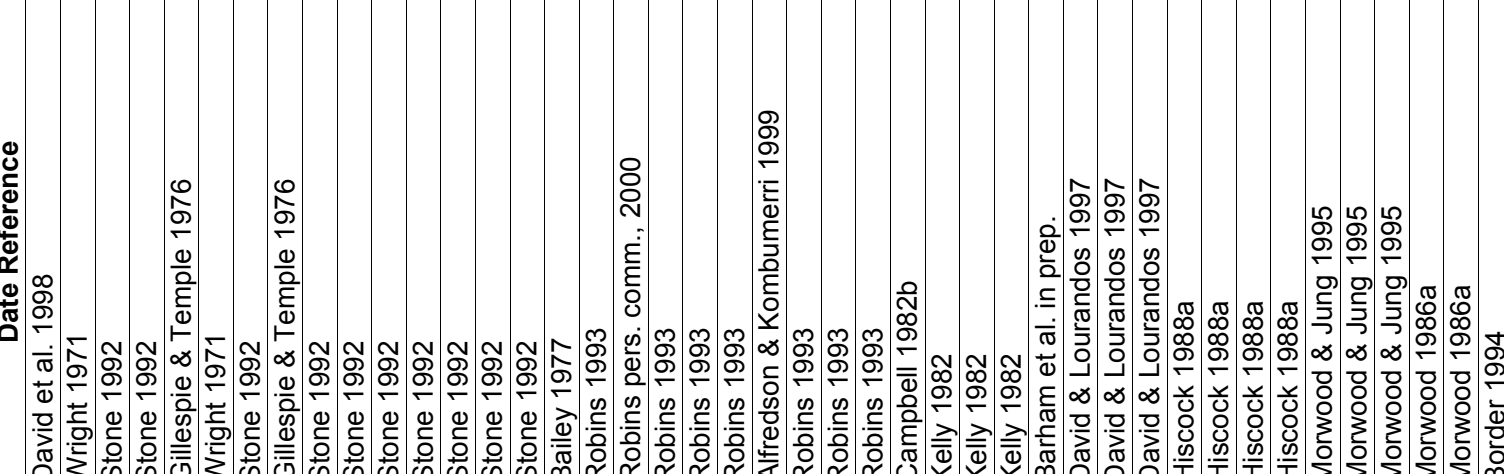

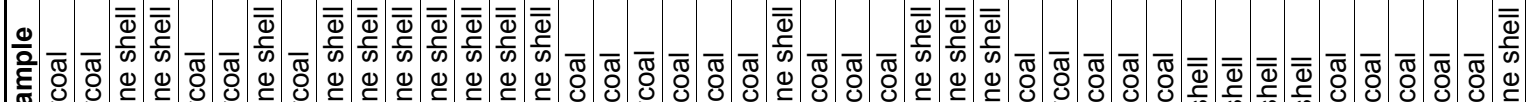

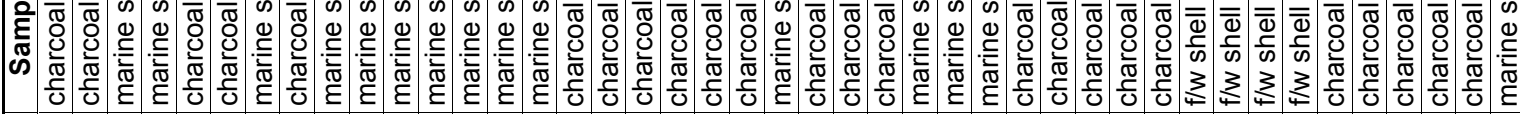

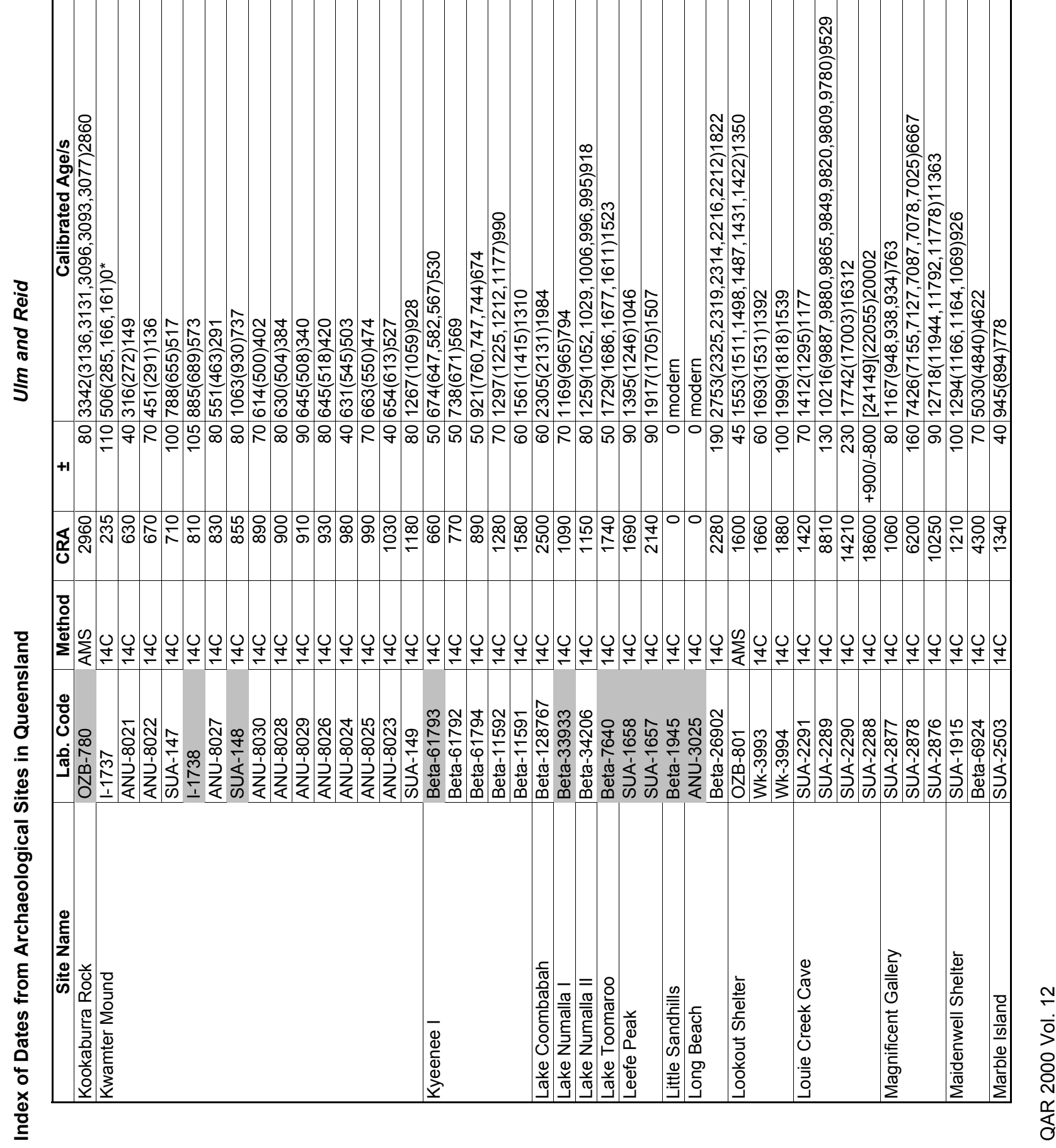


\begin{tabular}{l|l|l|l|l|l|l|l|l|l} 
& & & & & & & & & \\
\hline
\end{tabular}

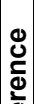

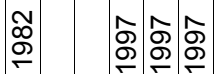

命

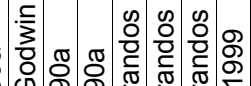

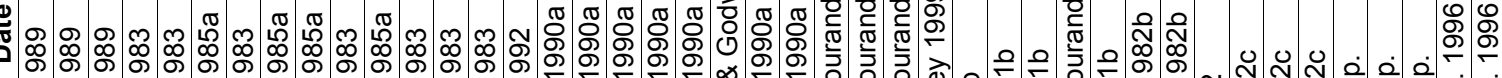

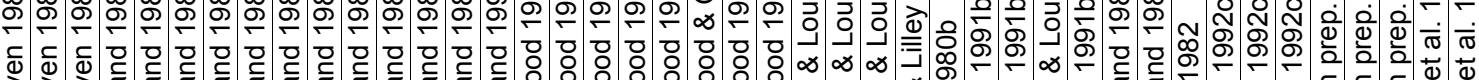

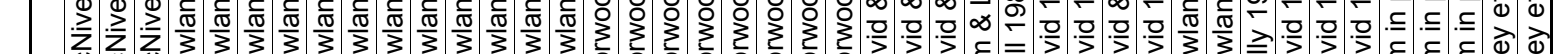

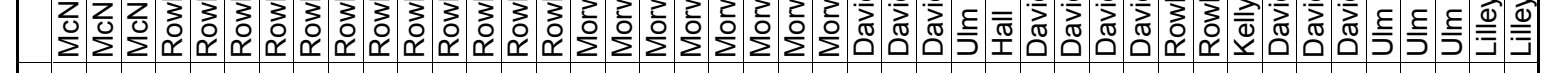

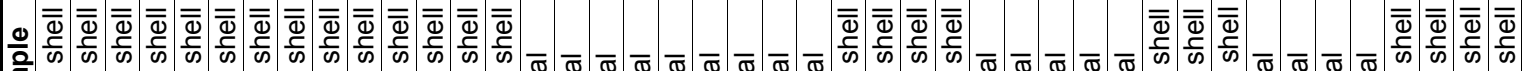

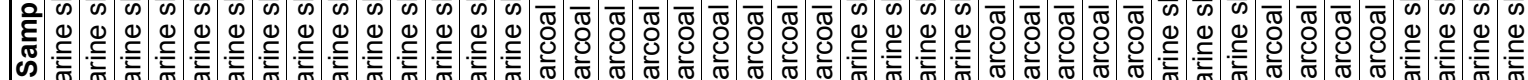
ڤ

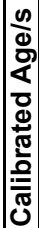

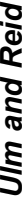

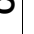

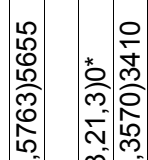

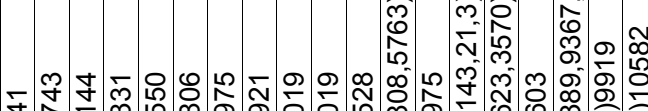

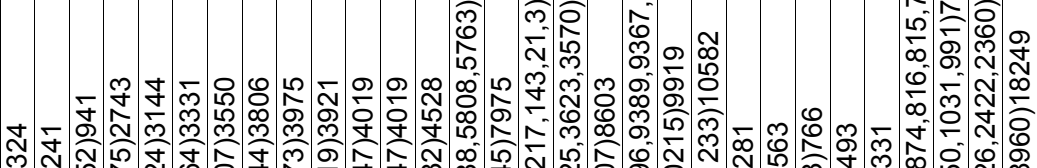

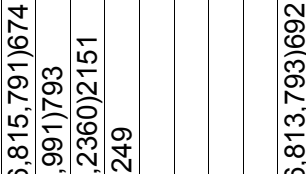

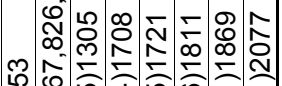
$\infty$

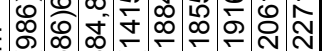
(

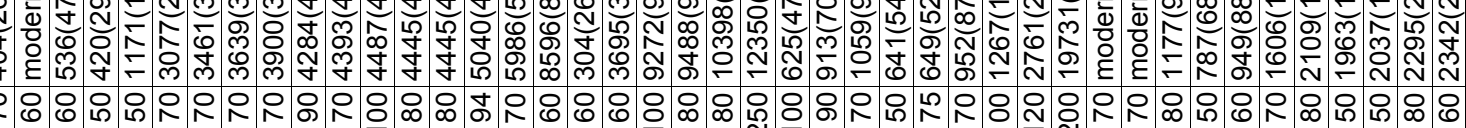

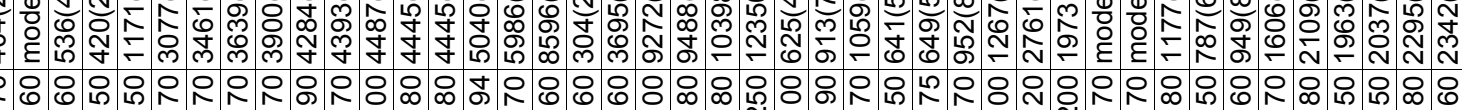
$+1$

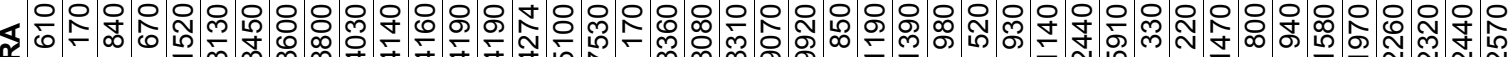
怘

¿

严

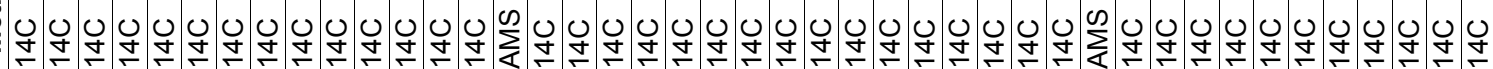

茴

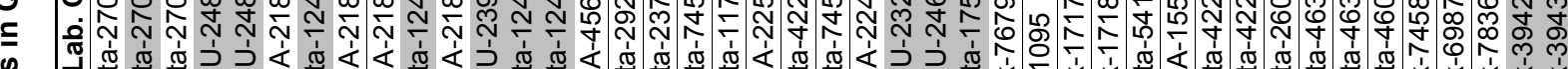

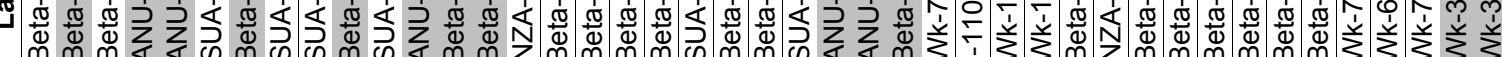

응

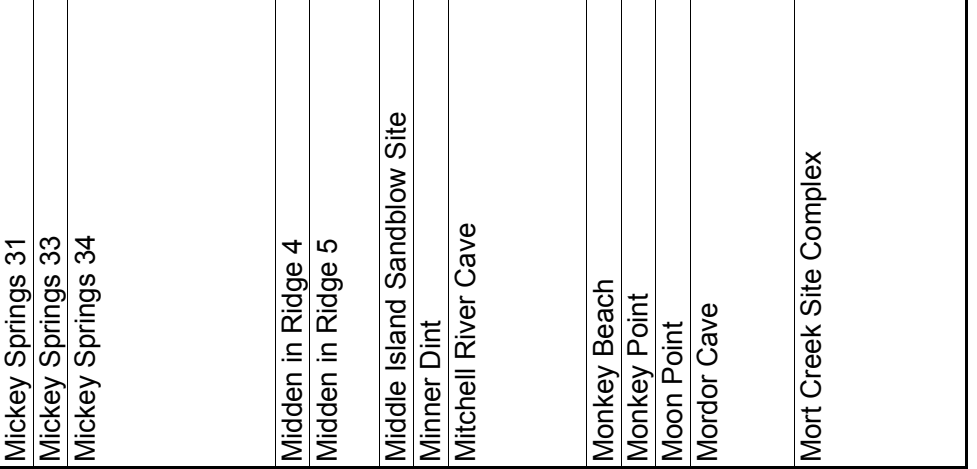

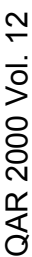




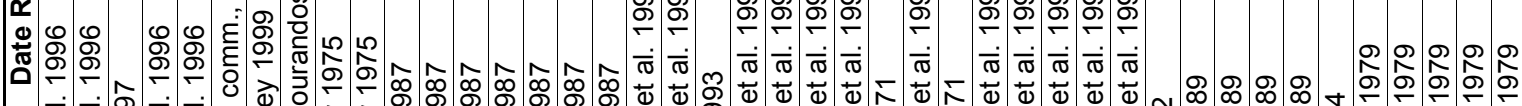

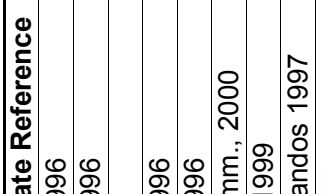

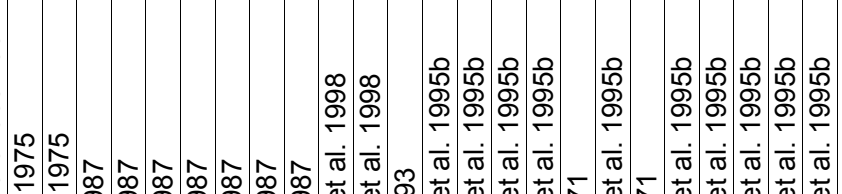

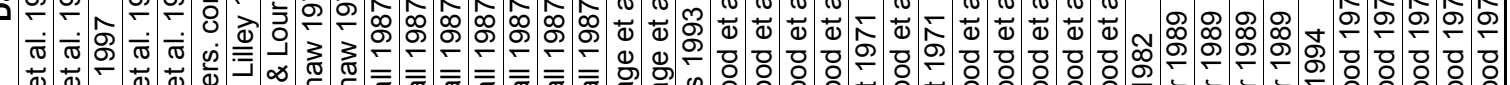

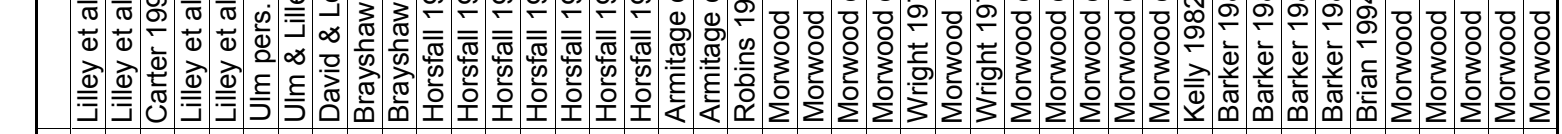

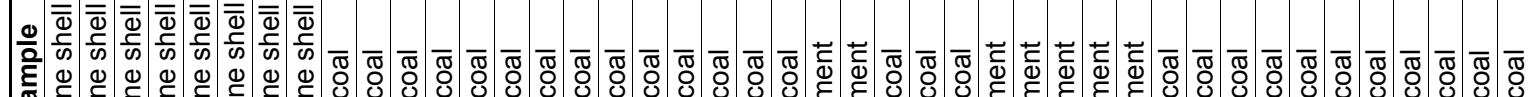

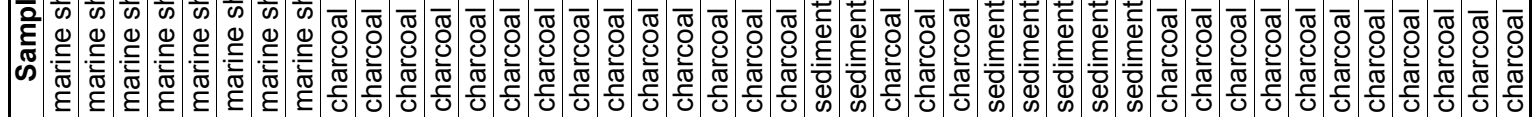

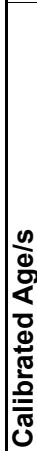

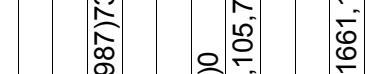

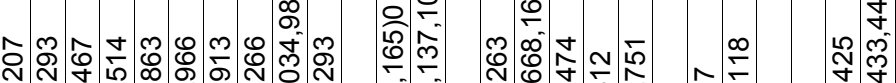

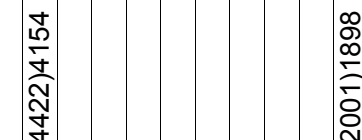

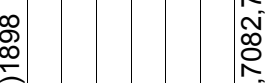

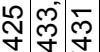

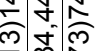

袋等

家

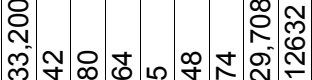

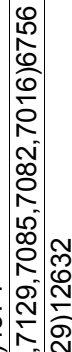

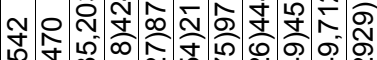

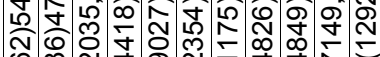
赵

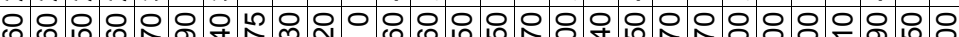

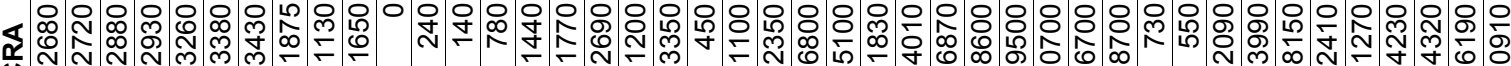
政

$\overline{8}$

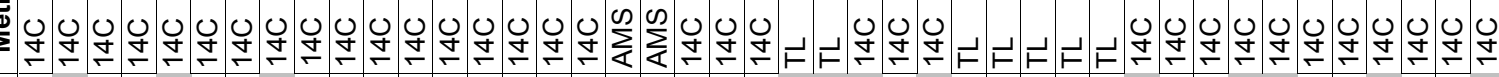

¿

-

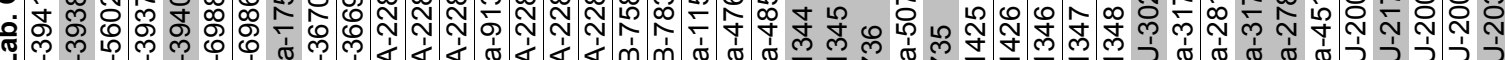

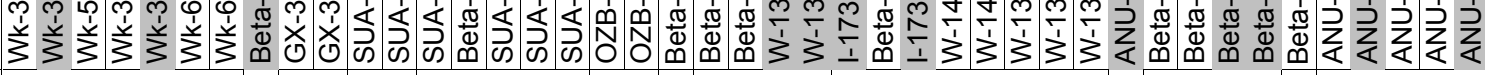

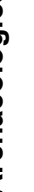

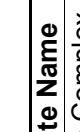

递

के

造

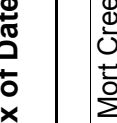

함

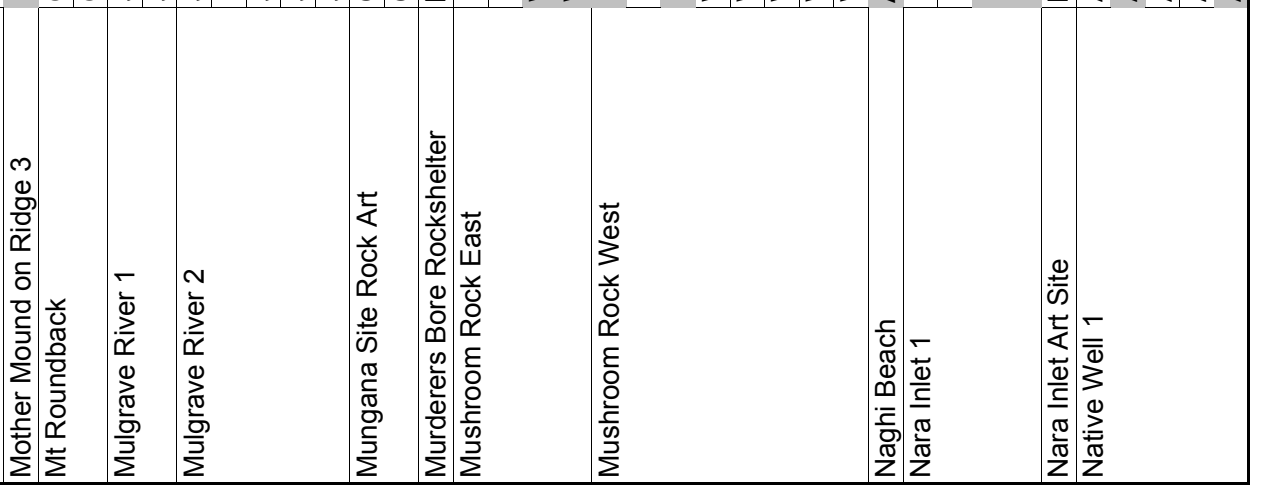


幽

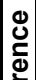

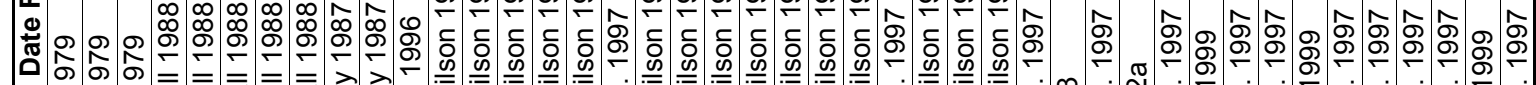

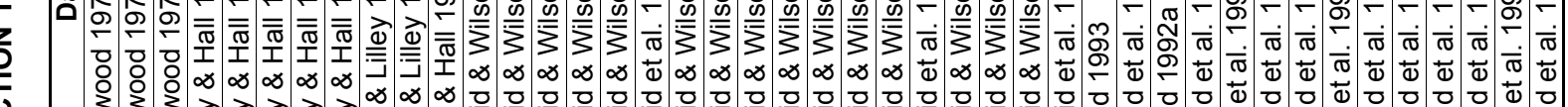

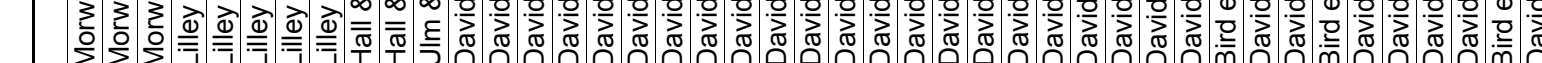

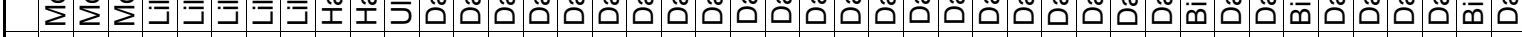

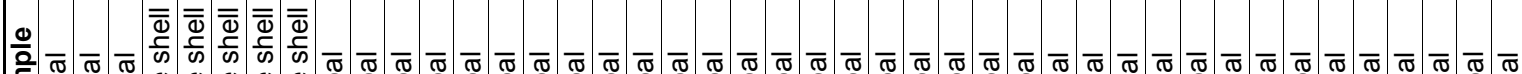

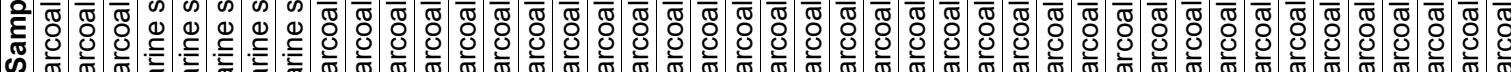

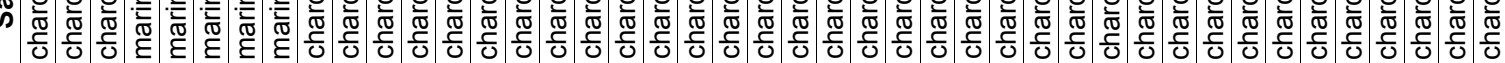

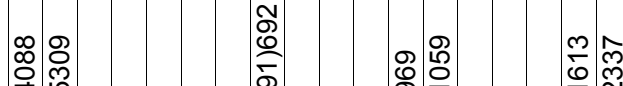

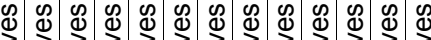

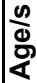

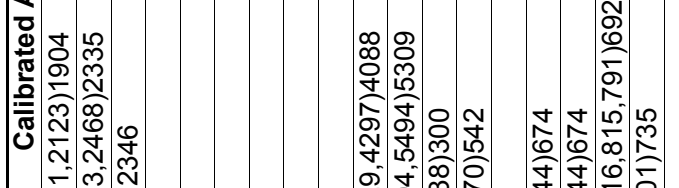

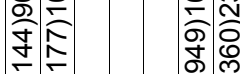

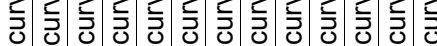

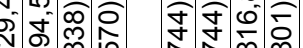

药

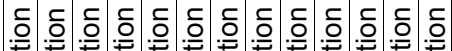

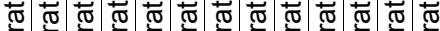

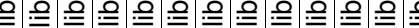

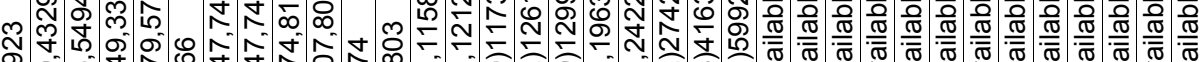

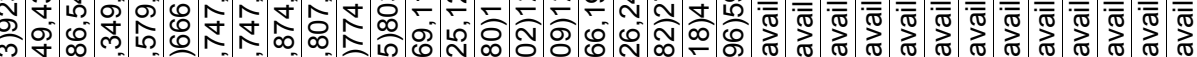
స พ

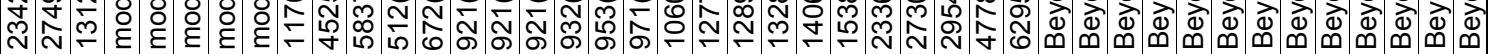
ஃஃ

$+1$

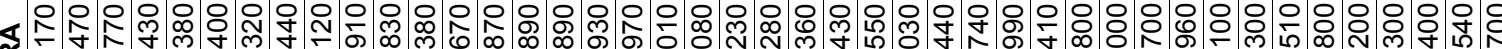
卒

:

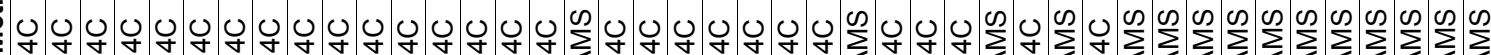

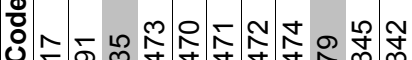

26)

8

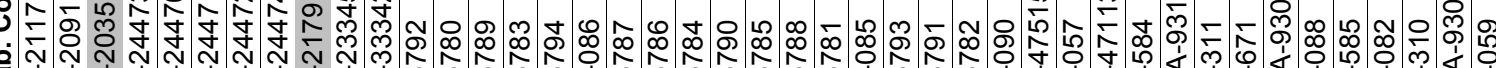

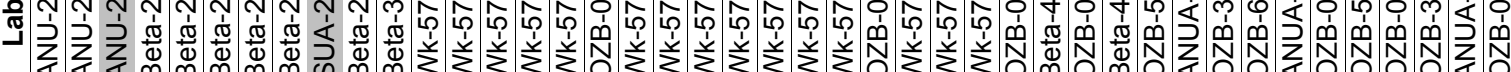

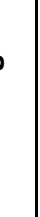

离

.

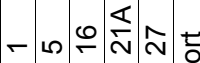

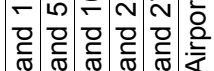
$\frac{\pi}{\omega} \frac{\pi}{\omega} \frac{\pi}{\omega} \frac{\pi}{\omega} \frac{\pi}{\omega} \quad 0$

每

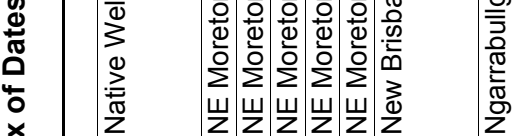




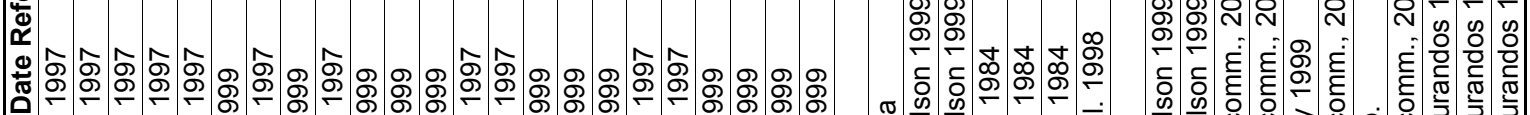

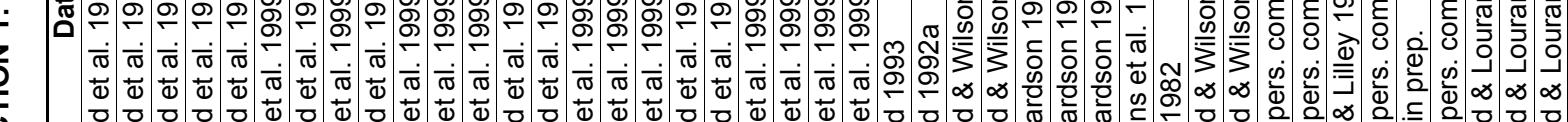
.0.

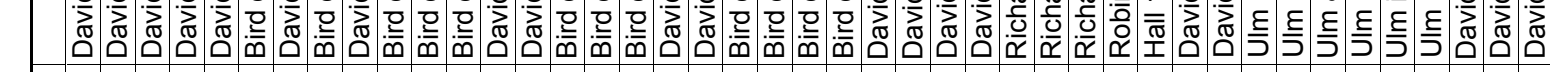

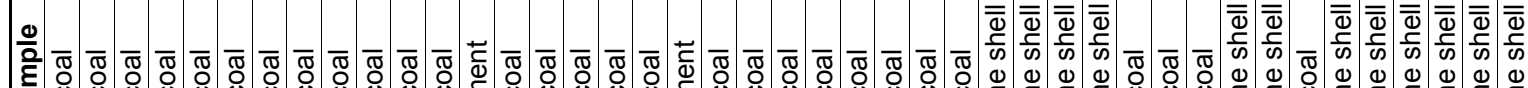

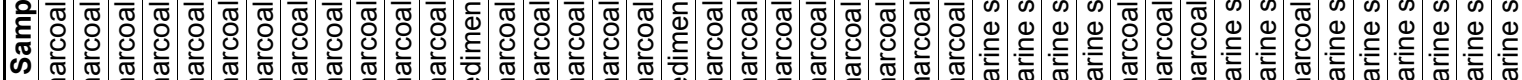
ஸे

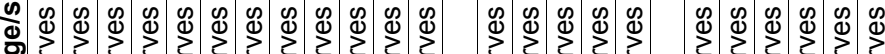

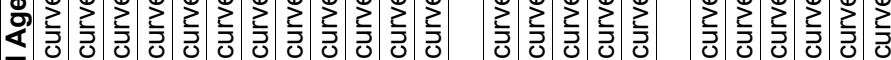

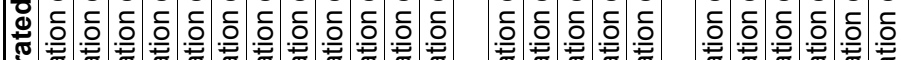

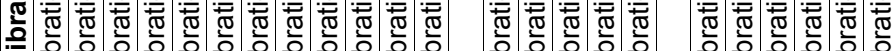

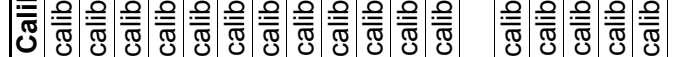

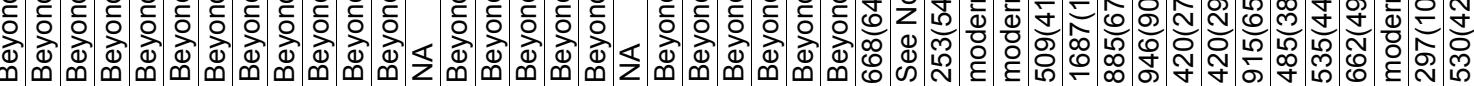

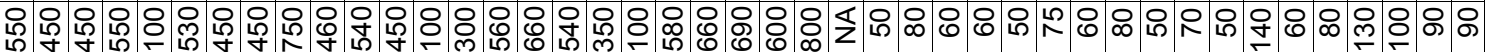
$+1$

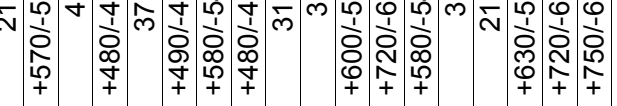

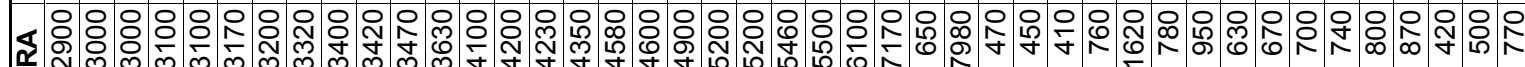

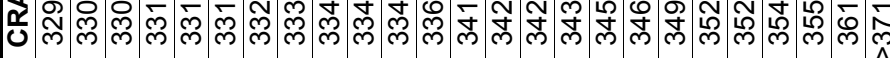

ס

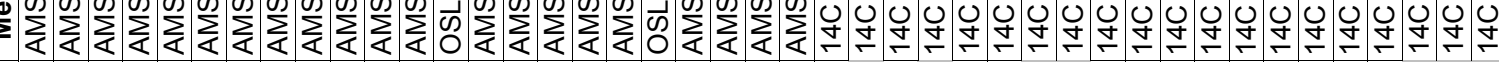

نे $\sum_{i}^{1}$

\section{4 은에}

\section{웅요}

Do

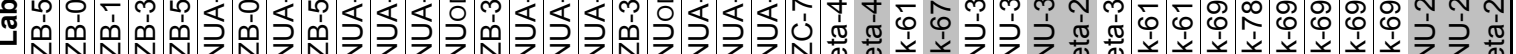

空 
نे

N

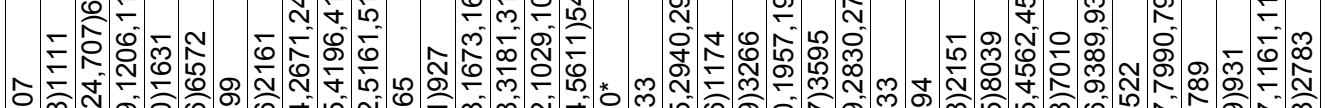

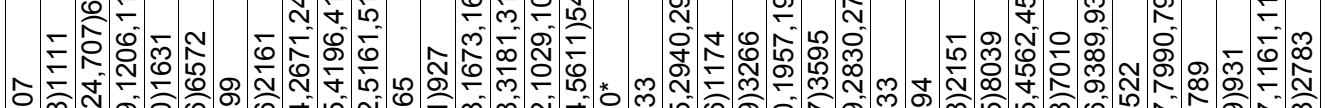

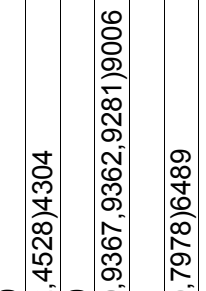

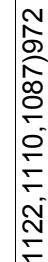

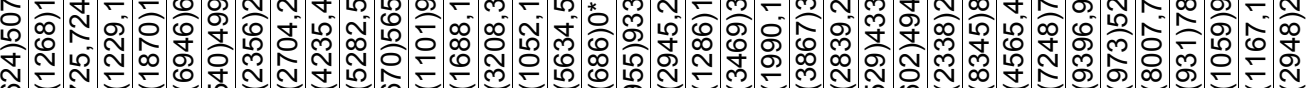

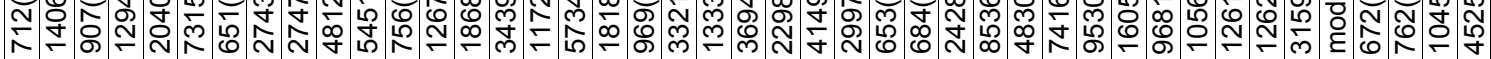

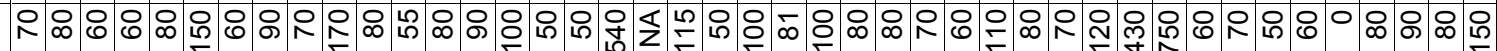
$+1$

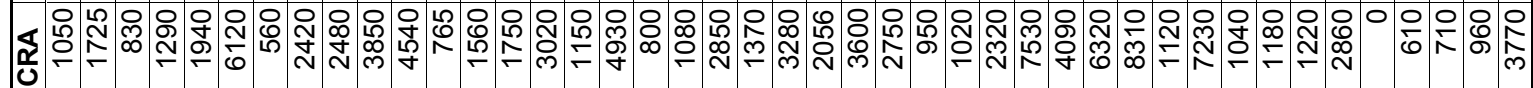

:

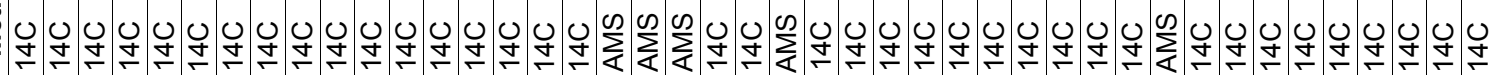

نำ

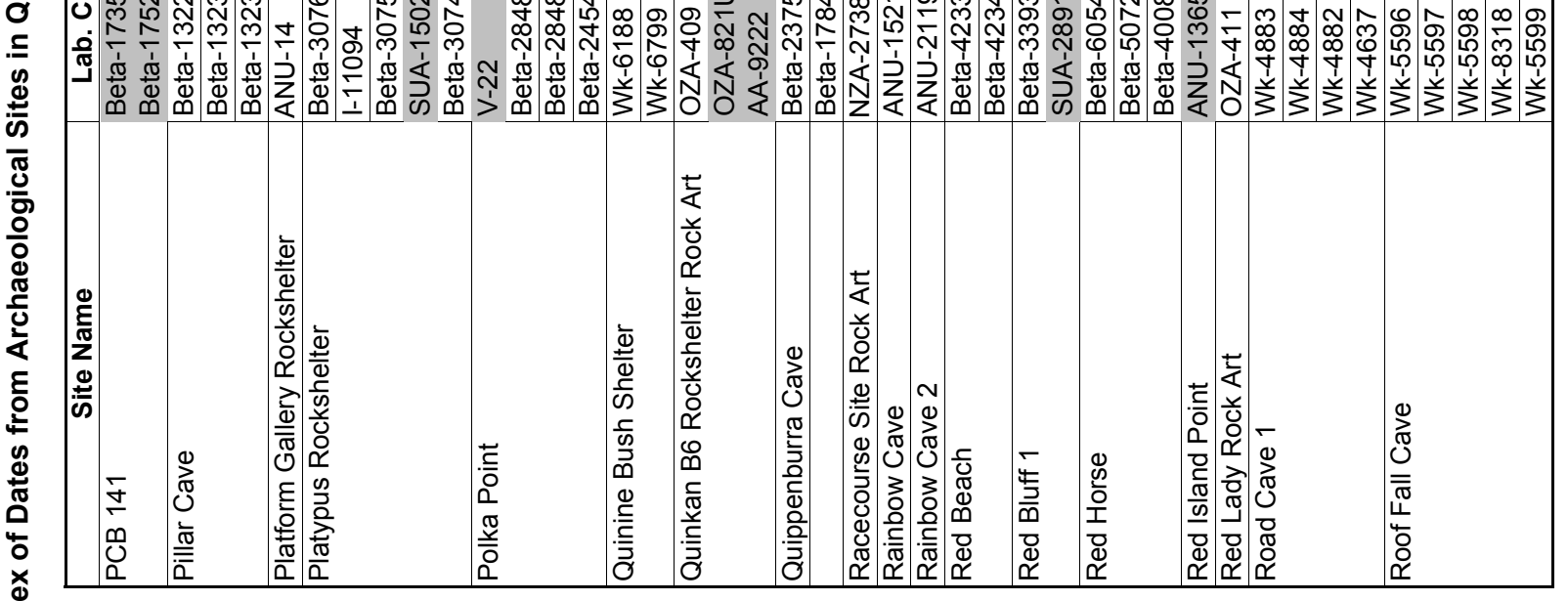

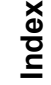




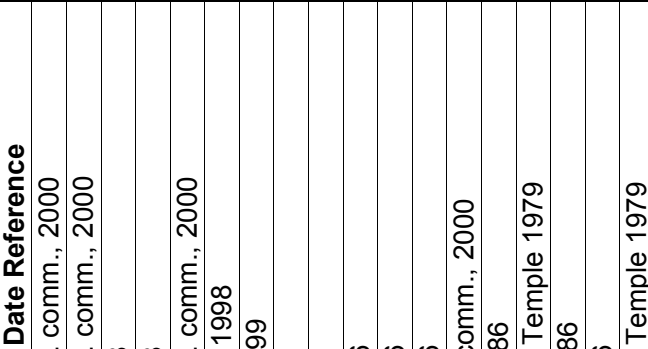

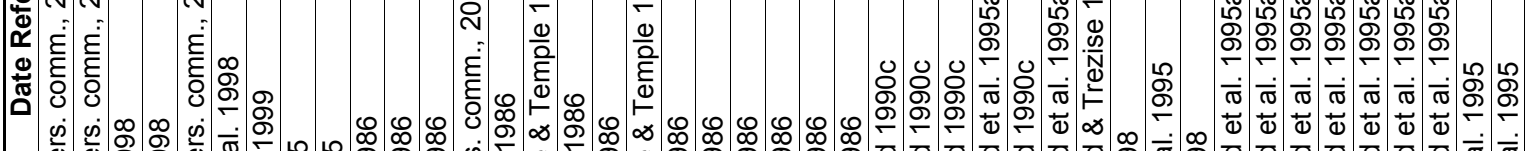

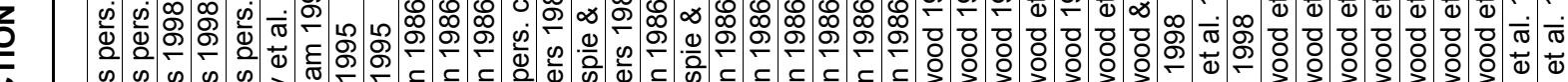

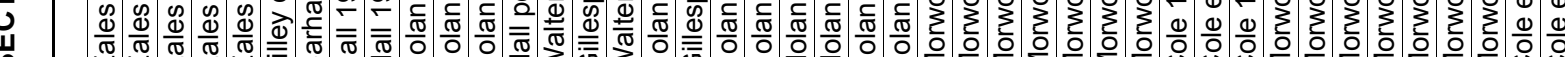

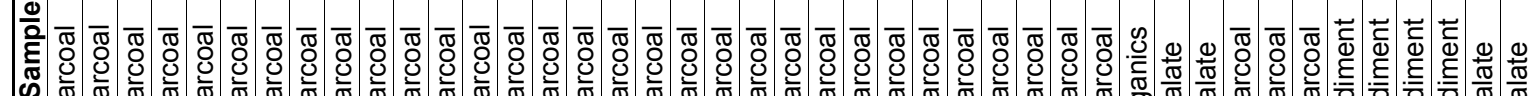

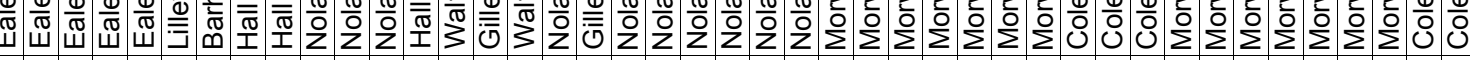

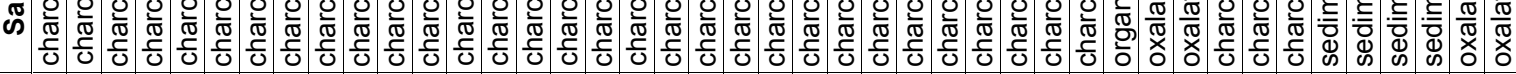

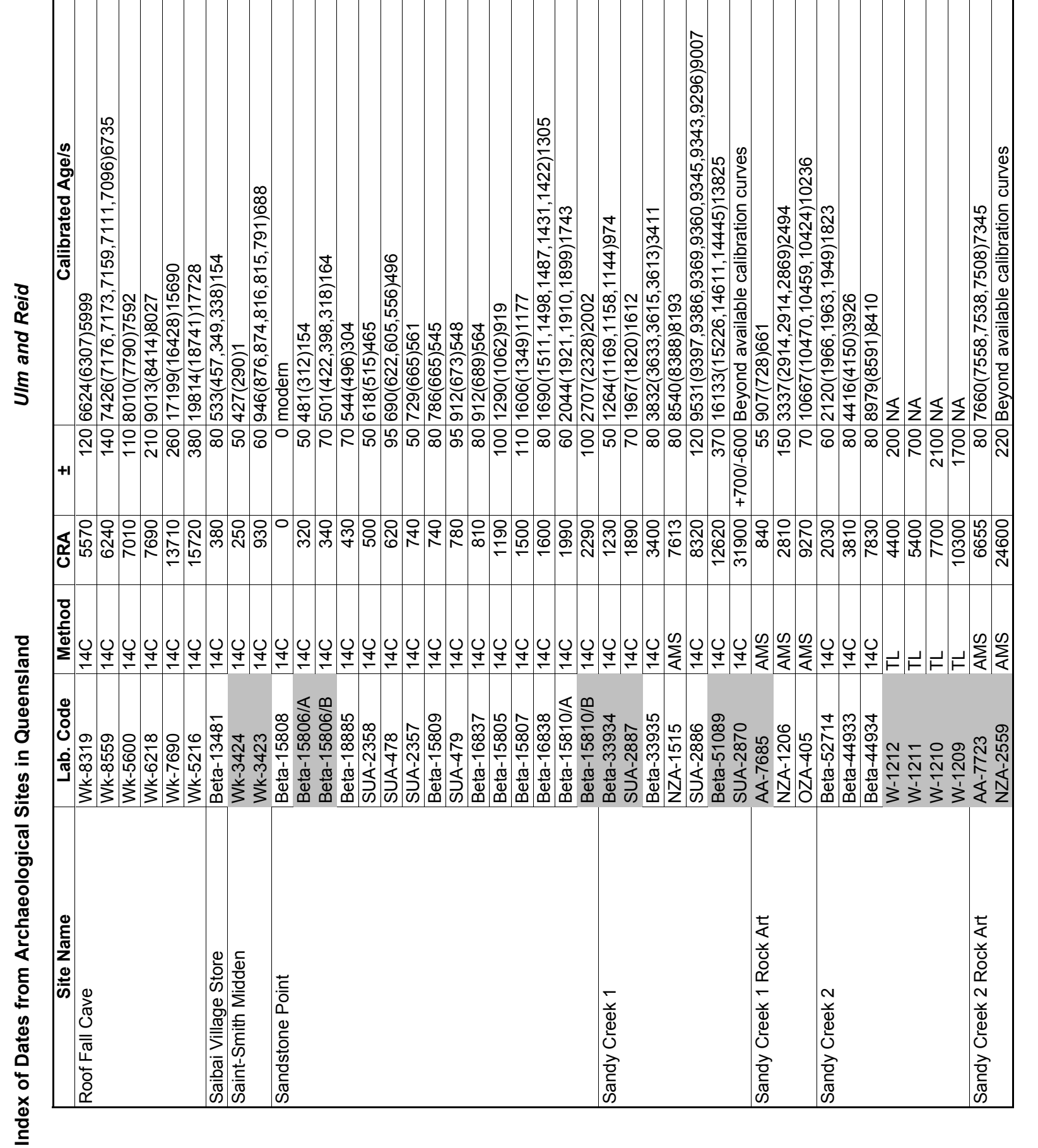




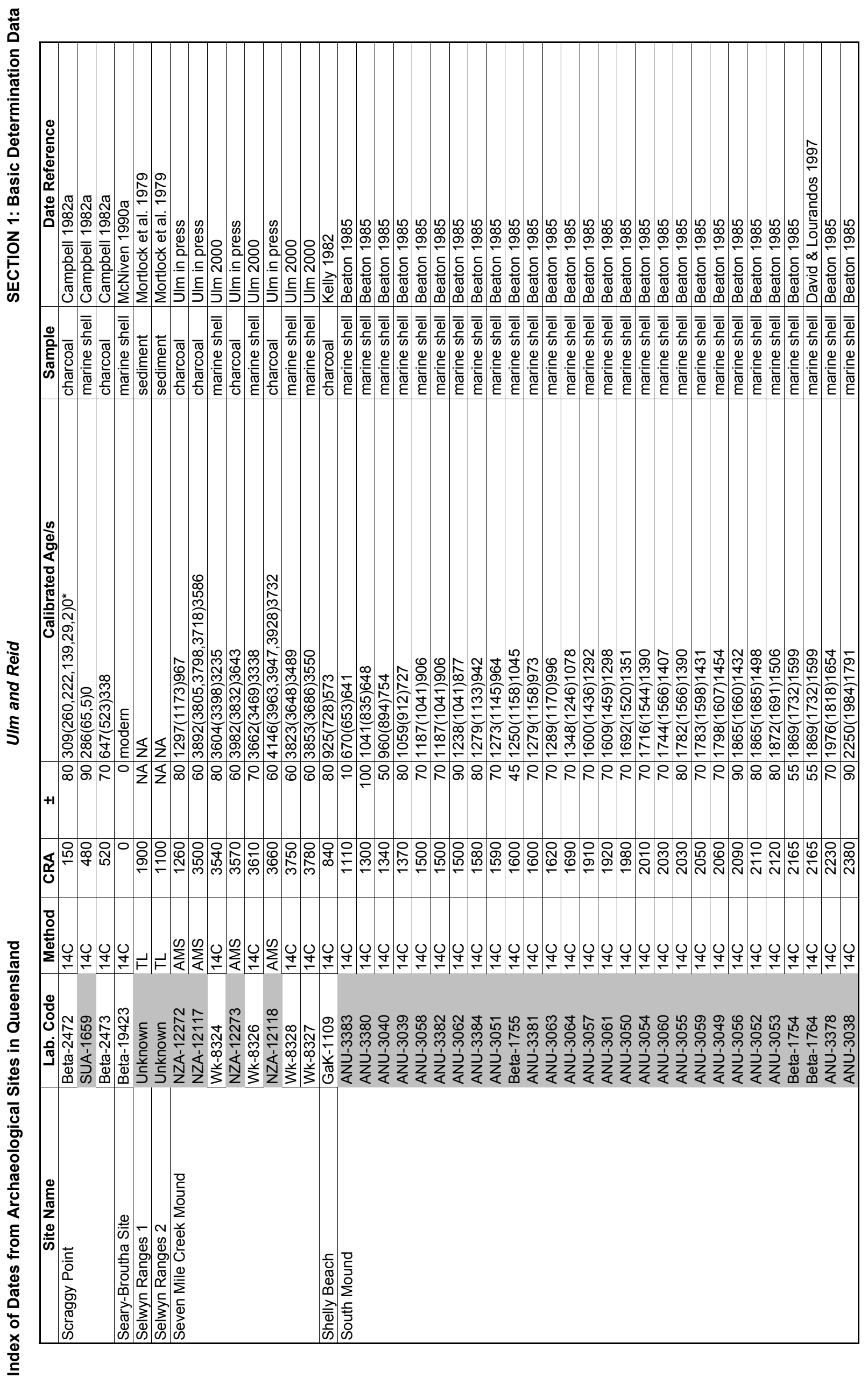




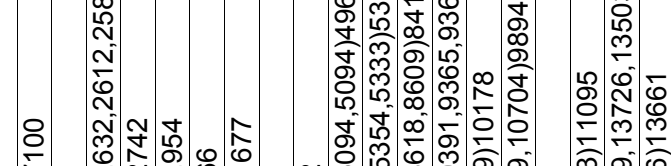

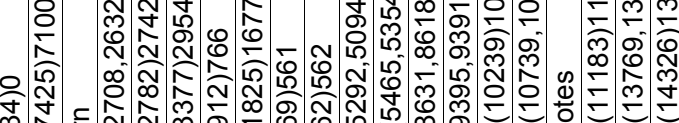

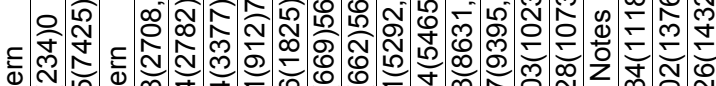

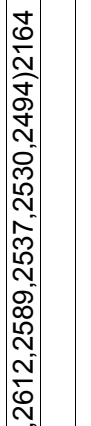

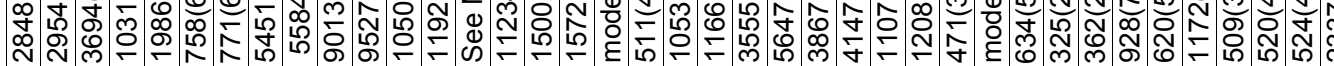
rs

$+1$

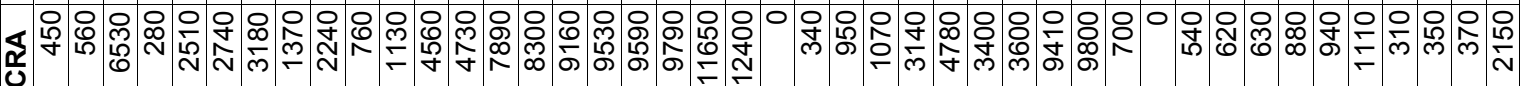

$\overline{0}$

$\frac{\text { D }}{\frac{0}{5}}$

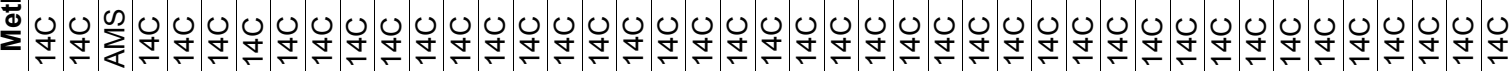

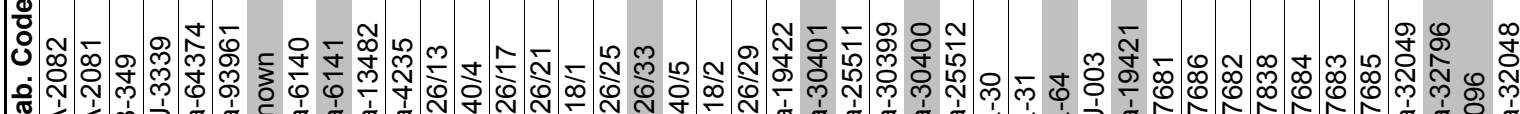

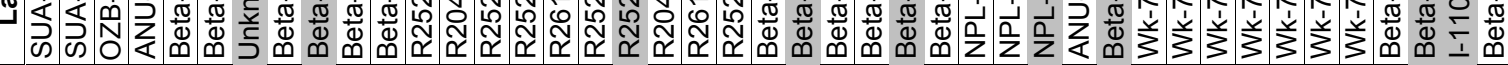

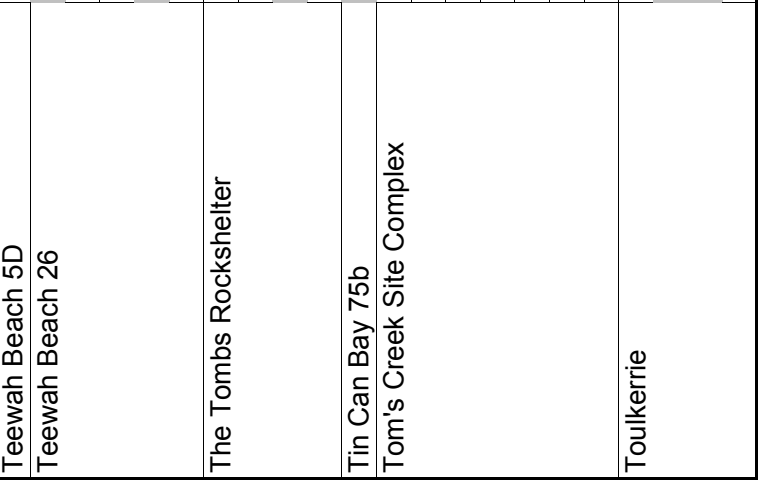




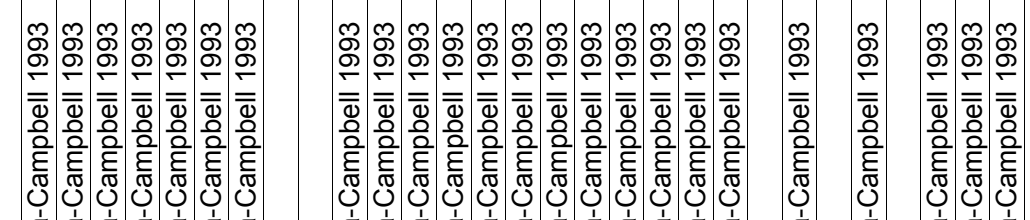

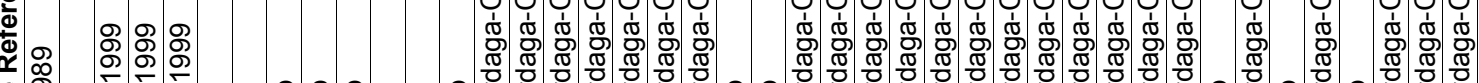

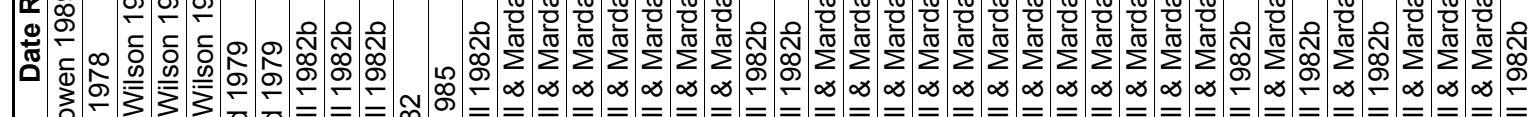

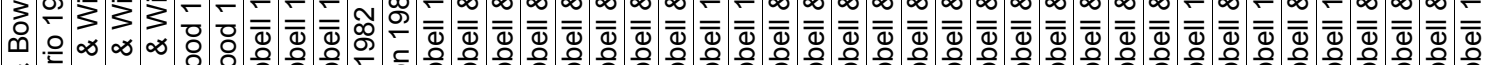

œ

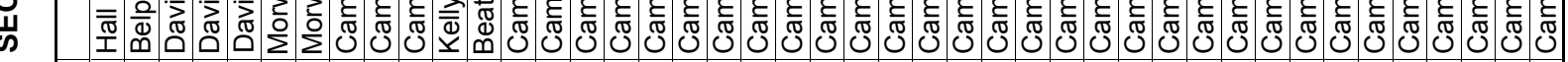

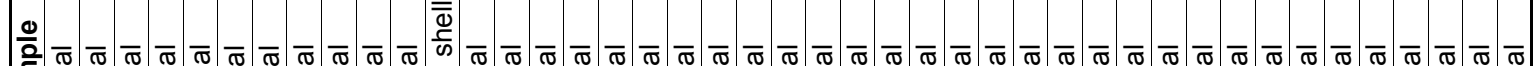

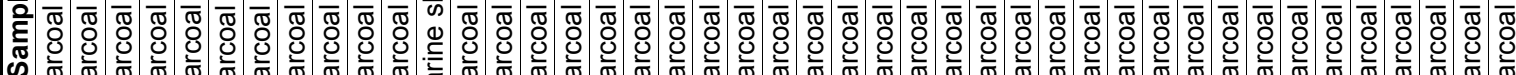

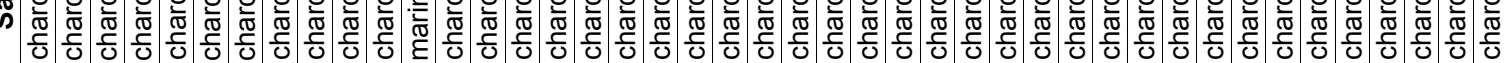

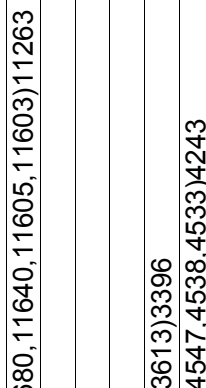

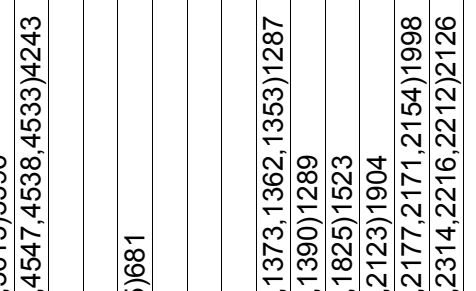

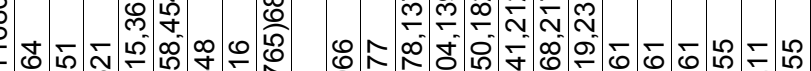

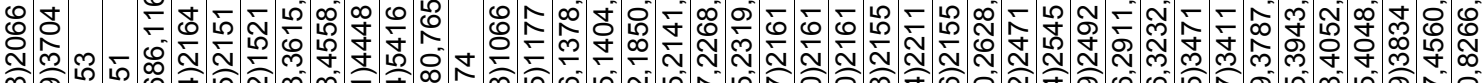
क人

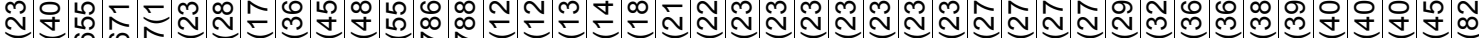
m.

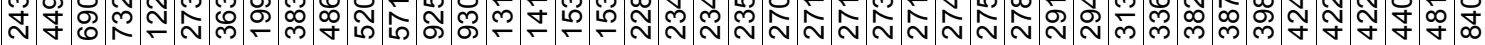

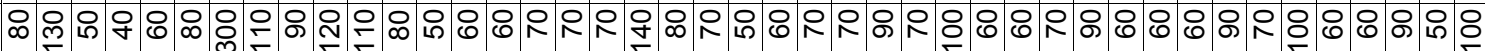

$+1$

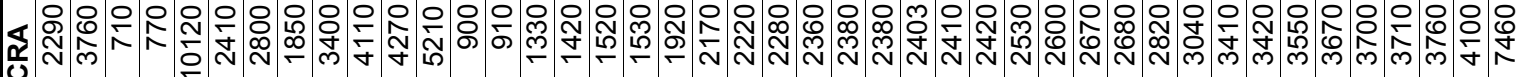

:

$\frac{\text { 을 }}{\frac{0}{0}}$

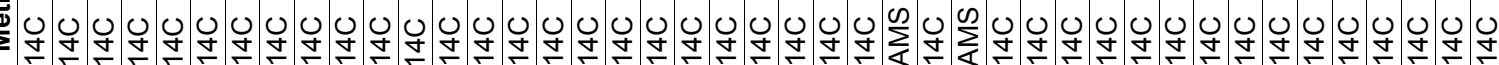

¿ें

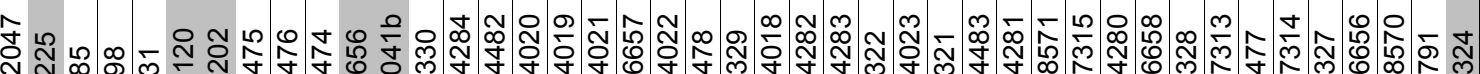

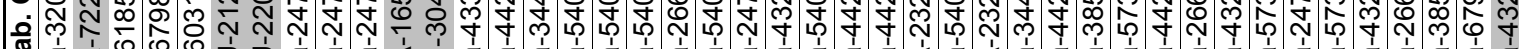

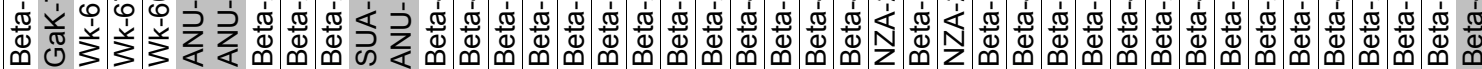

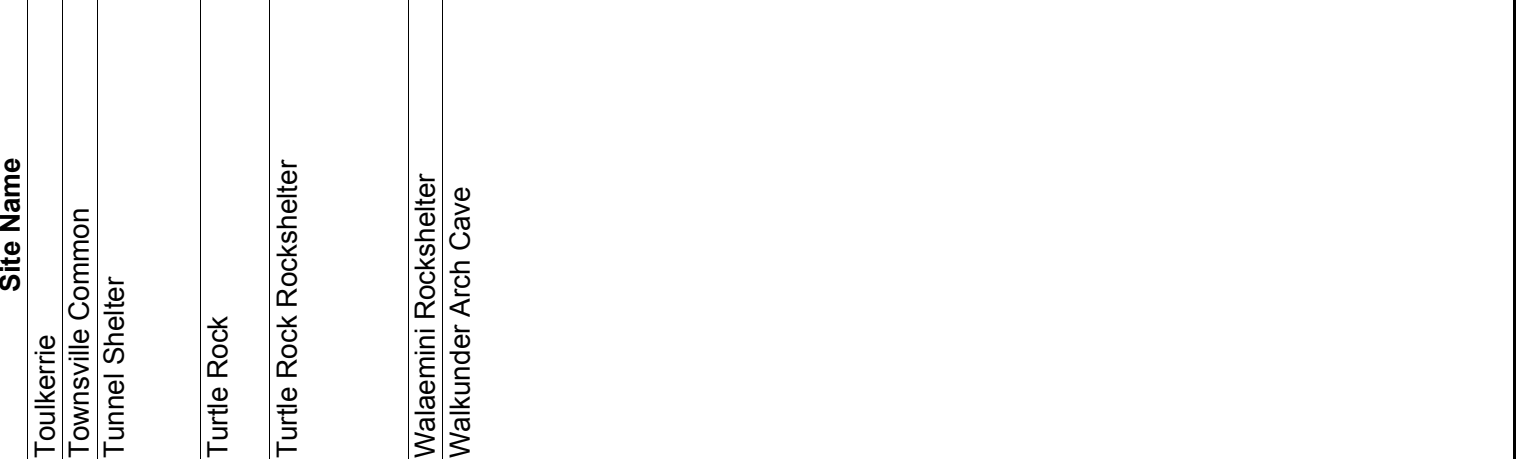


Øூூֶ:

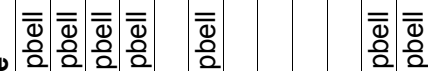

\&

ర్ల

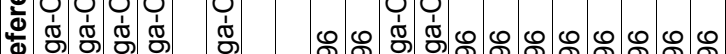

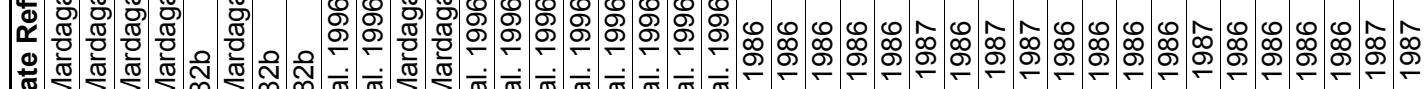

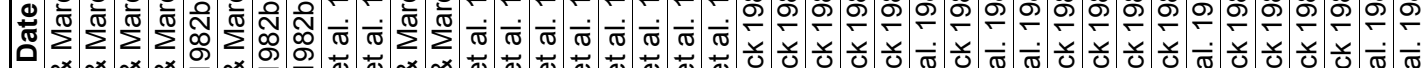

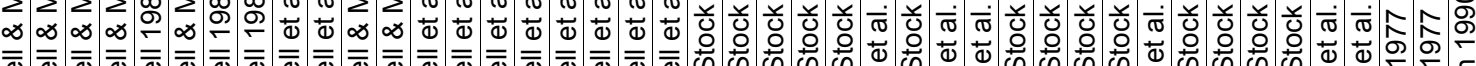

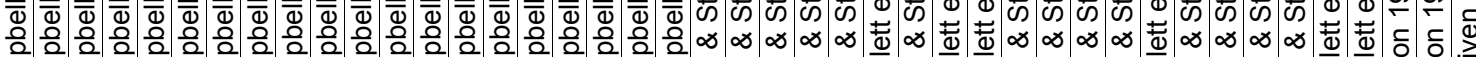

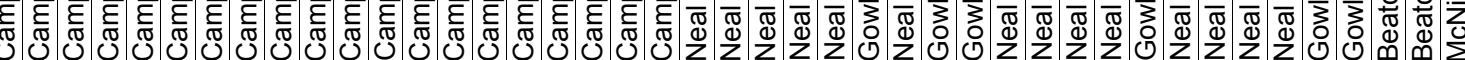

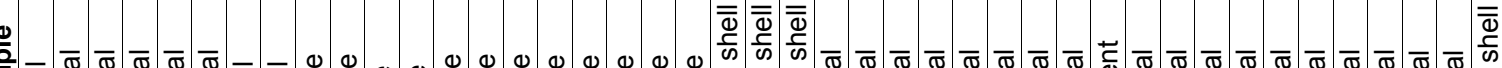

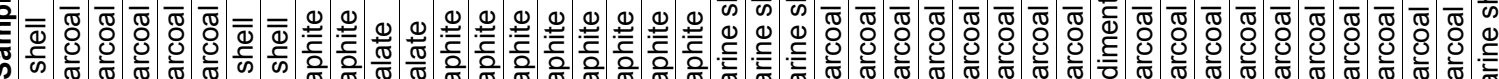

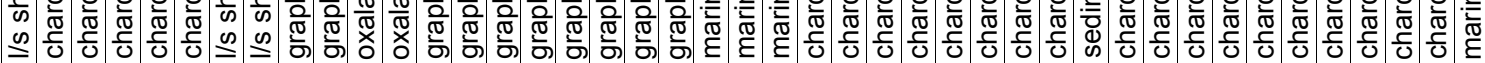

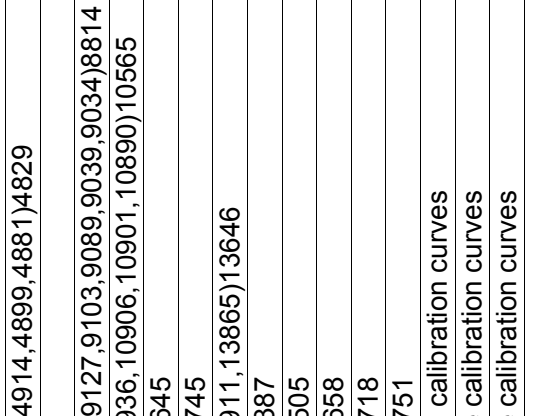

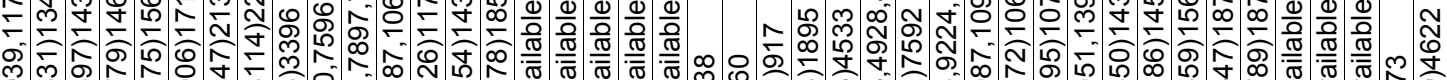

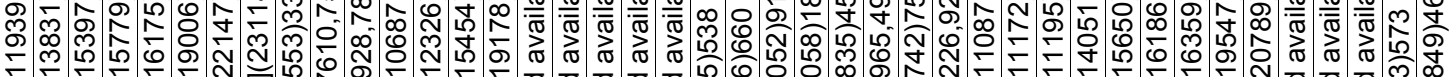

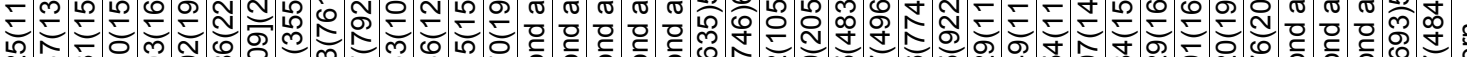

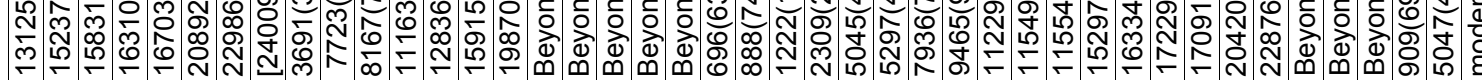

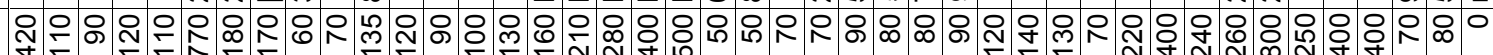
$+1$

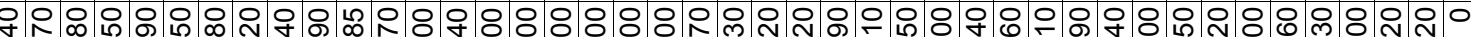

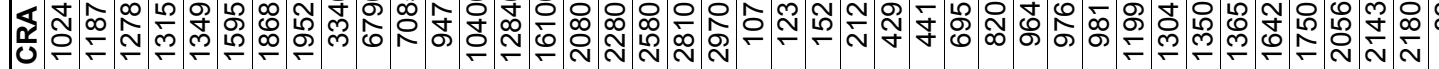

을 O

$\frac{8}{8}$

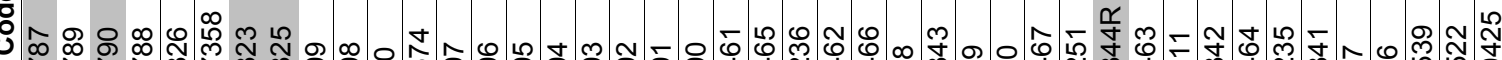

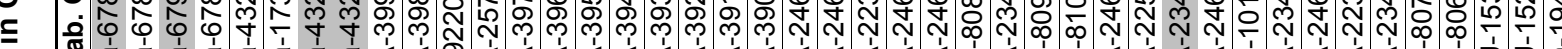

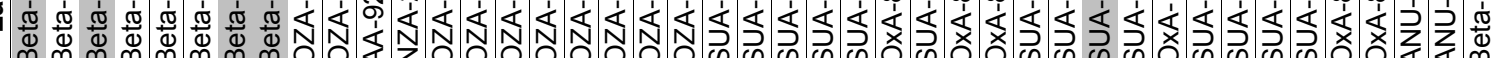

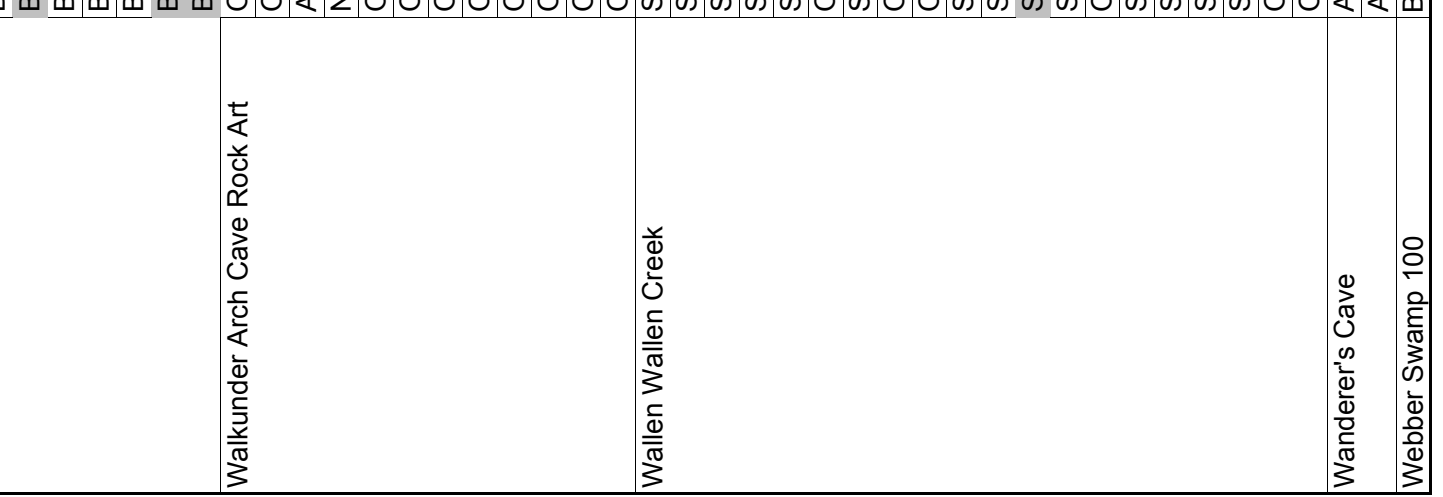




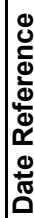

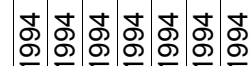

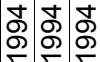

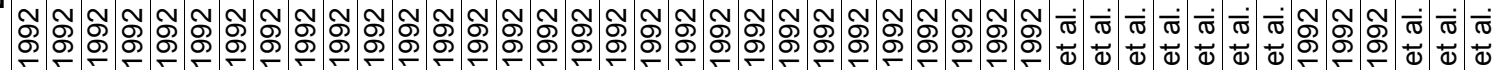

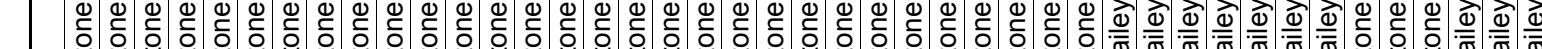

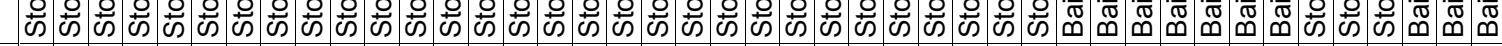

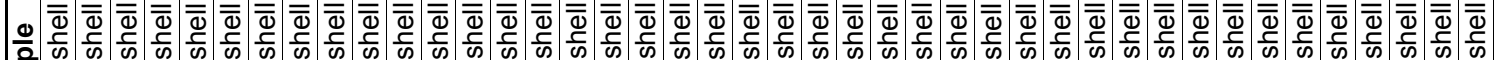
心)

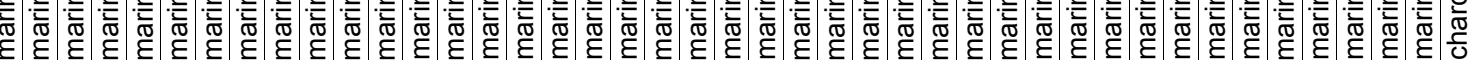

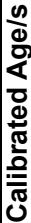

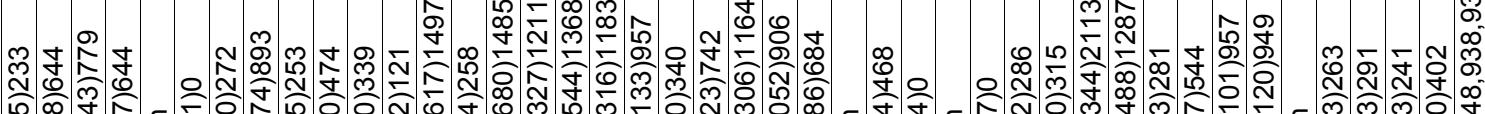
15 象 50

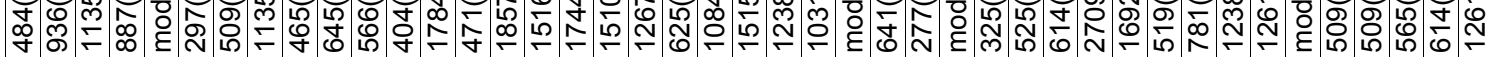

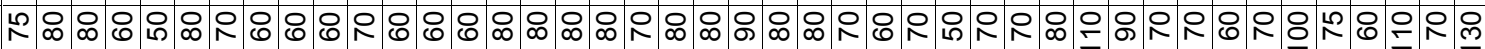

$+1$

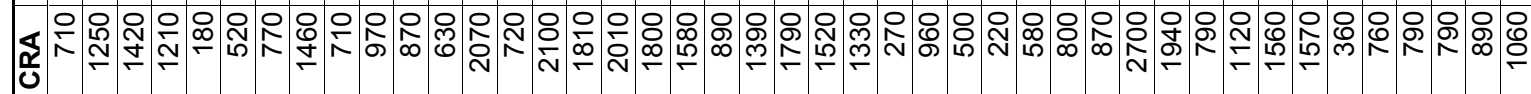

을

달

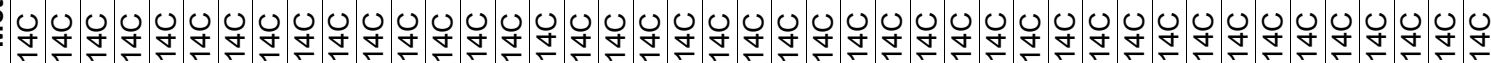

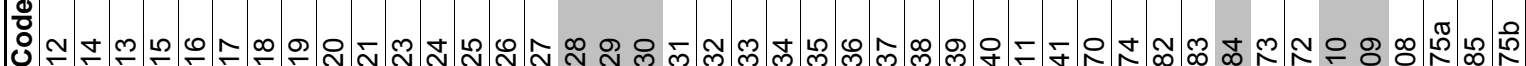

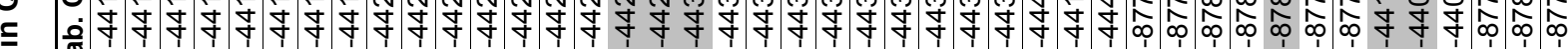

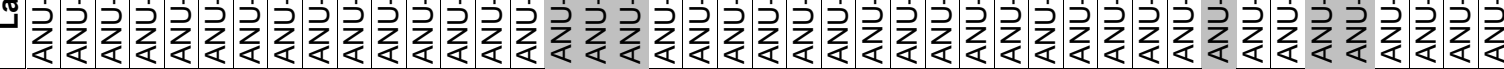
传

政

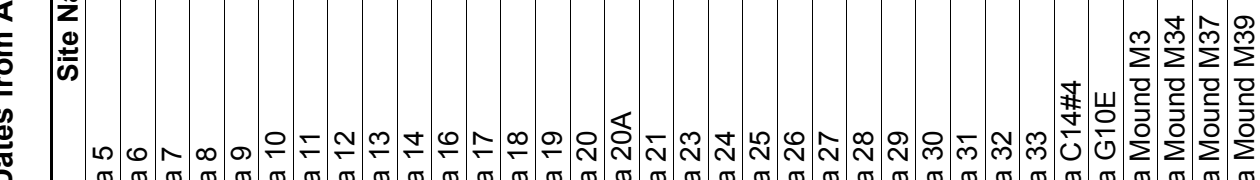


$\stackrel{9}{ \pm}$

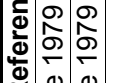

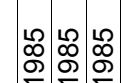

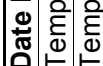

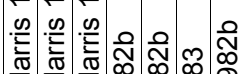

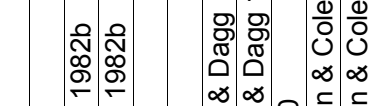

レ̊:

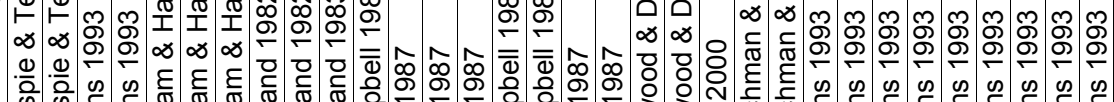

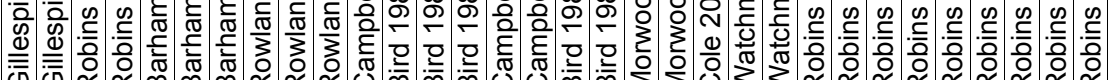

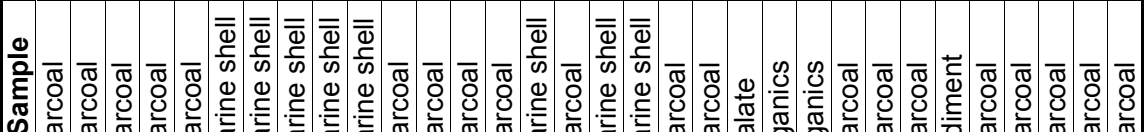

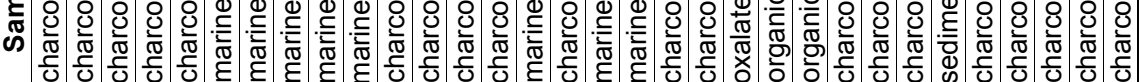

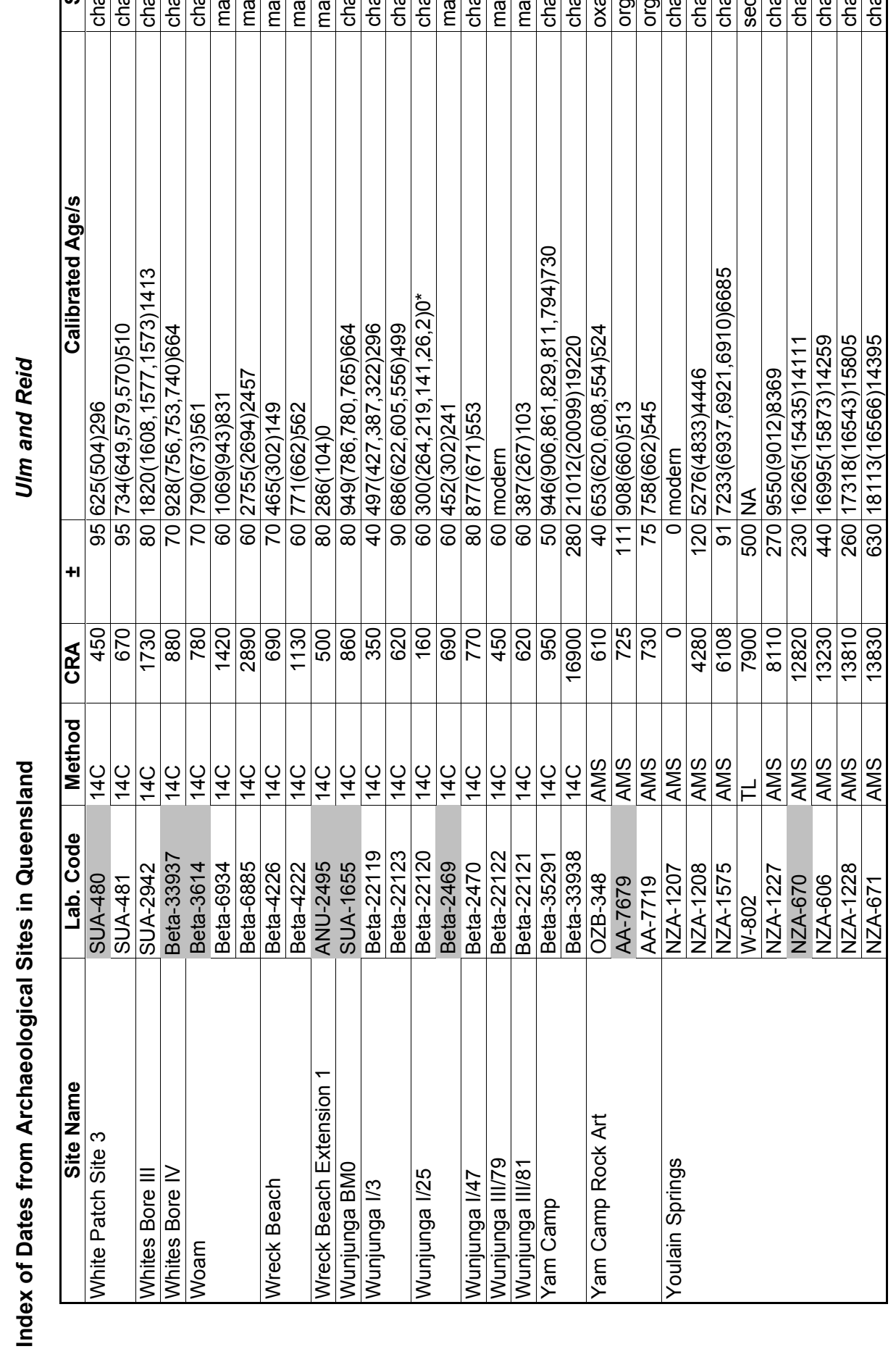




\section{SECTION 2:}

Advanced Determination Data 
$1+\frac{1}{2}$

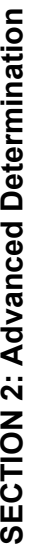

\begin{tabular}{|l|l|l|l|l|l|l|l}
+1 & & & & & & & \\
\hline
\end{tabular}

:

0.0 .15 .1

ดे ู่ :

$+1$

เก ก $\stackrel{\bullet}{\dot{+}}$

这

-

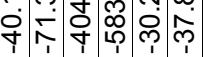

ָั

$+$

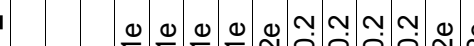

พุ.

$\stackrel{\infty}{\sim}$

$\frac{0}{0}$

Ð

+1 $\quad$ م

nitar

$\frac{d}{2}$

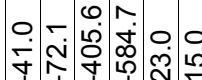

产.

章

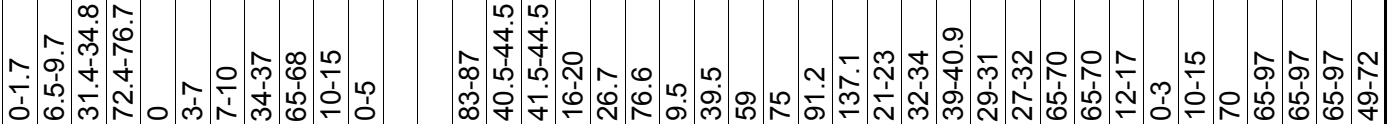

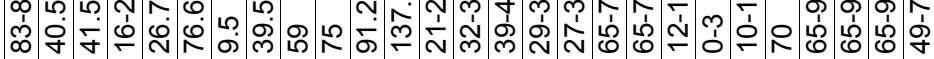

$\vec{x}$

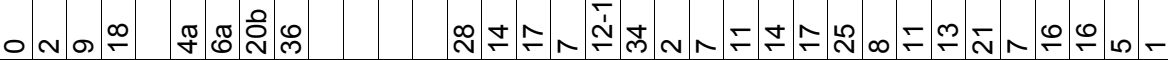

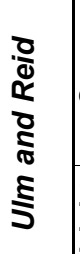

紊

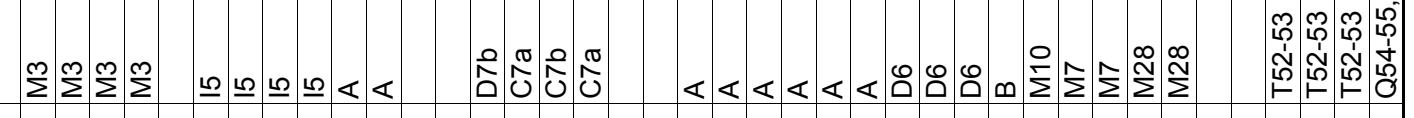

蒙

L

\$.

n) $m$

ह

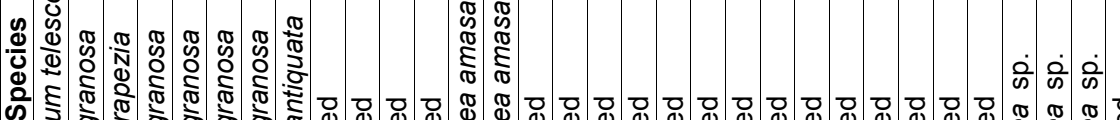

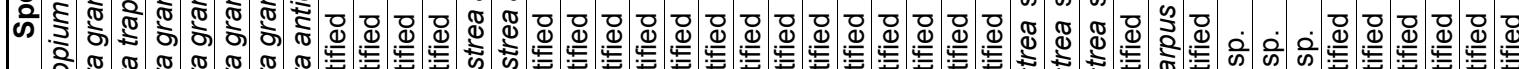

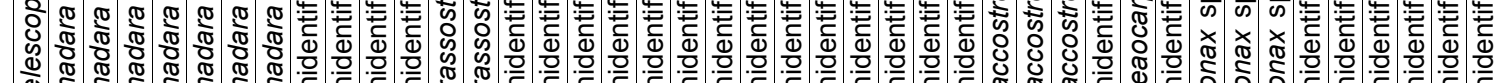

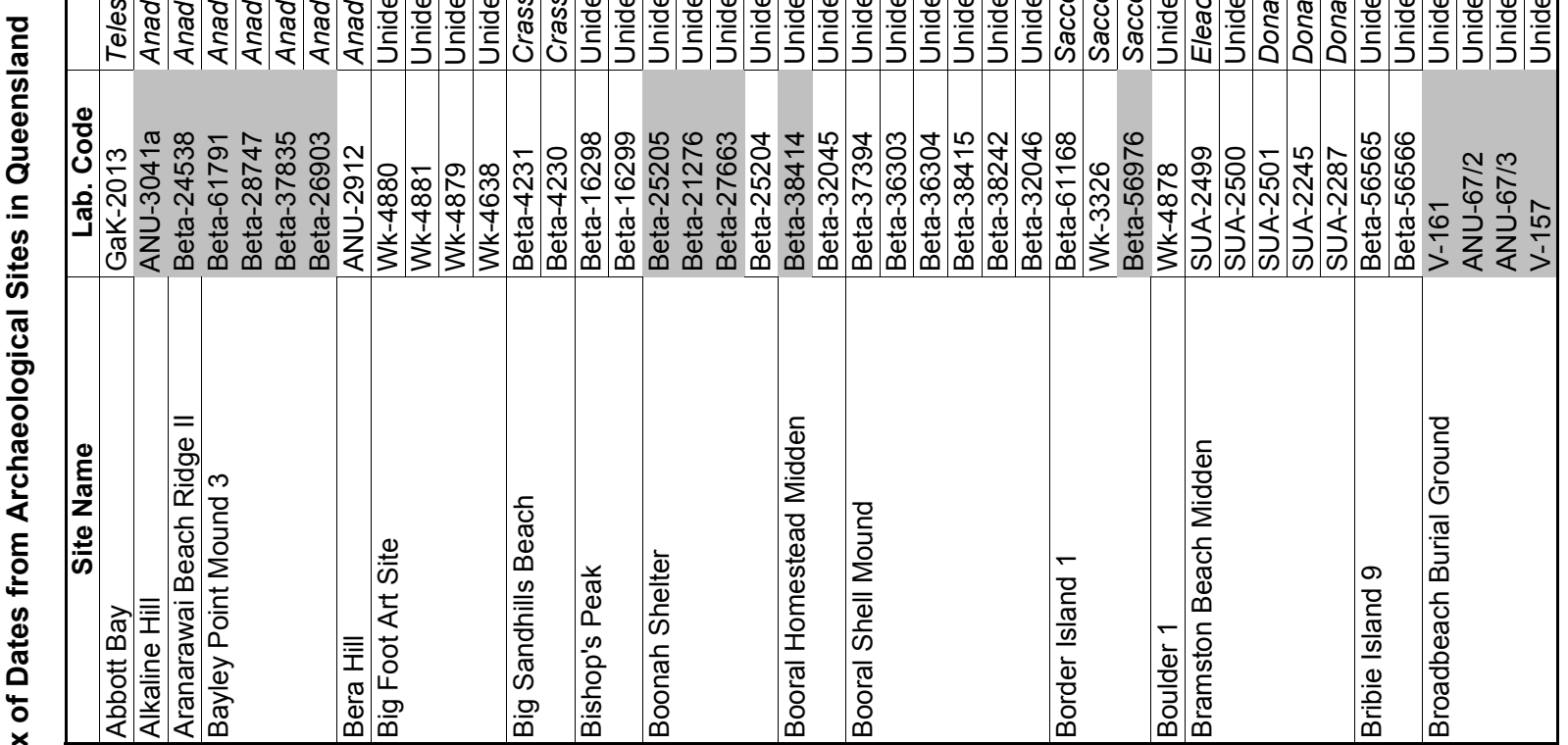




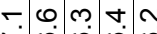

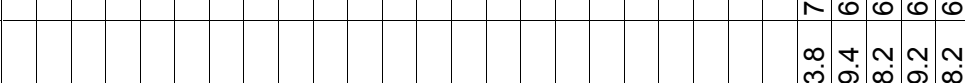

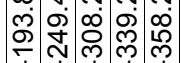

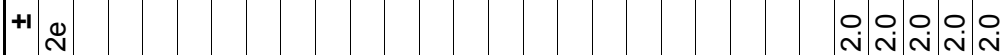

ن.

ठั

市

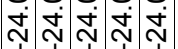

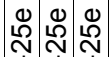

$+1$

$\frac{d}{d}$

产

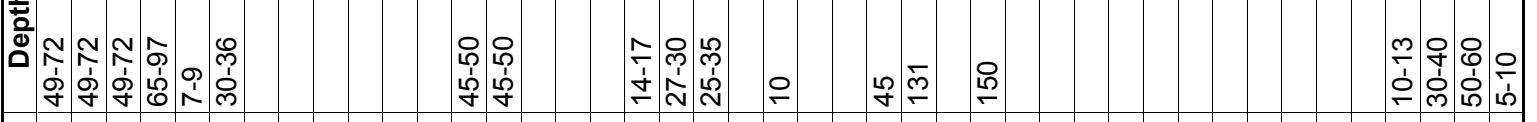

$\vec{x}$
n:
๗)

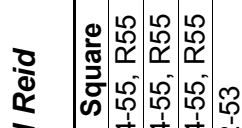

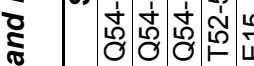

$\frac{10}{\omega}$

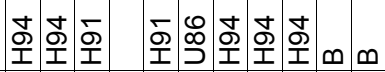

蒿

กำ

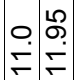

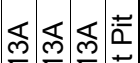

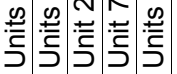

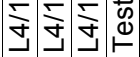

$\stackrel{0.00}{3}$

م

เ $\llcorner\llcorner$

$\frac{\mathscr{c}}{\mathrm{e}}$

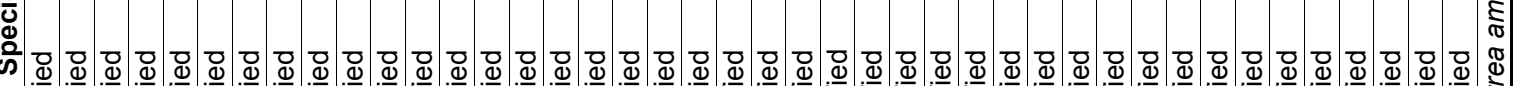

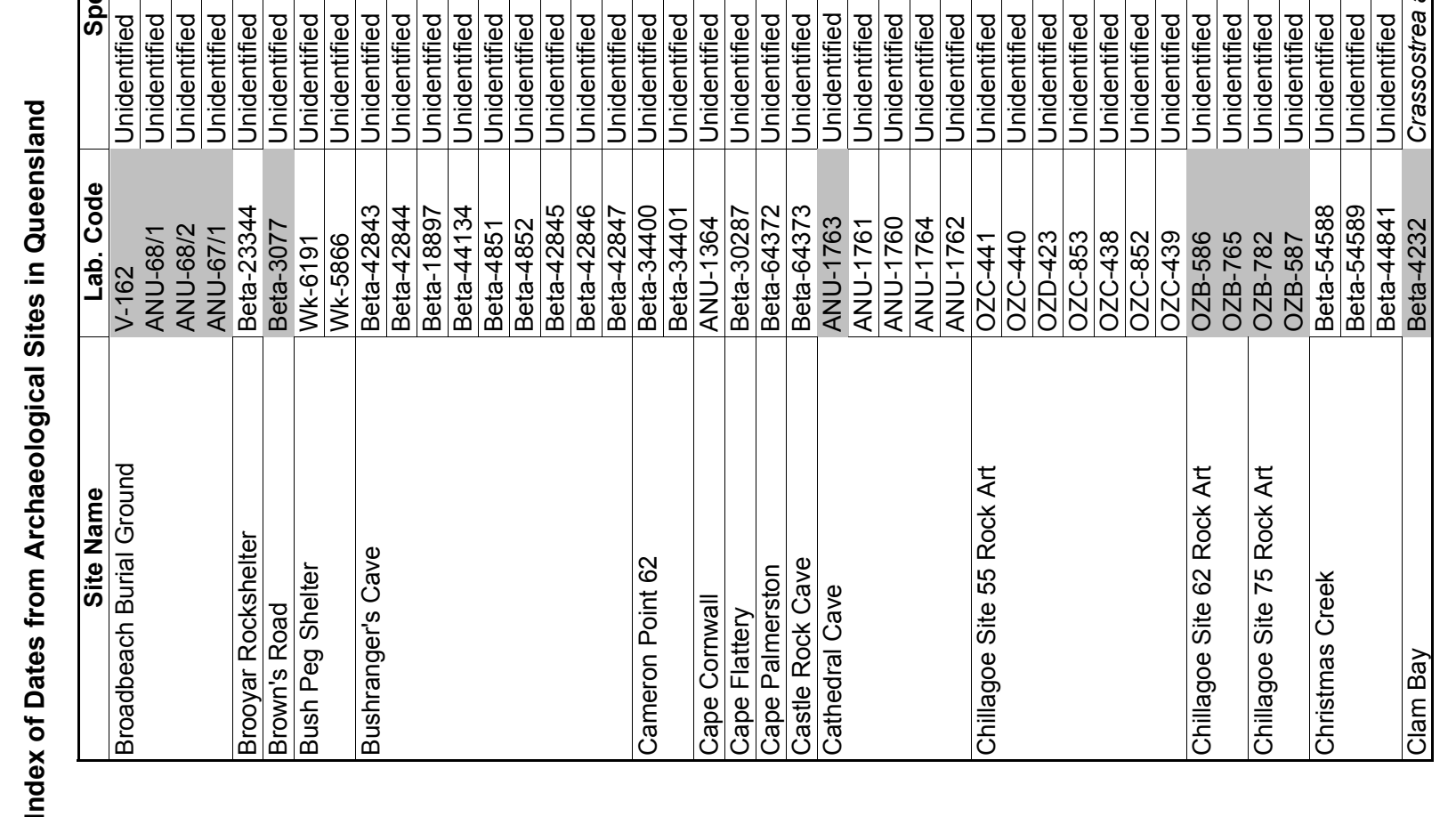




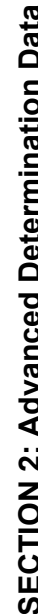

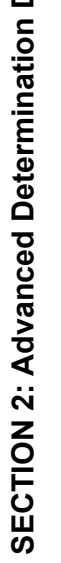

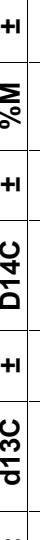

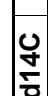

立

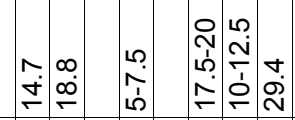



ํำ

$\vec{x}$

nNamnm

هి

:

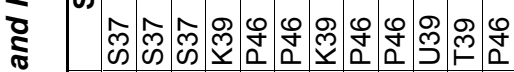

$0-$ ommm- 000 o.

욖옳

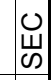

క

蒿

N

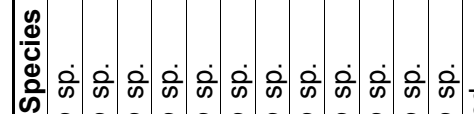

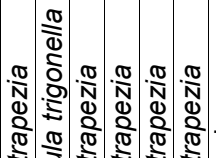

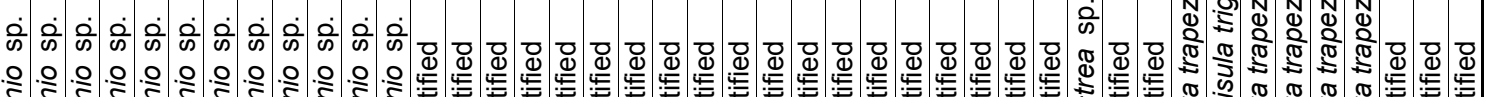

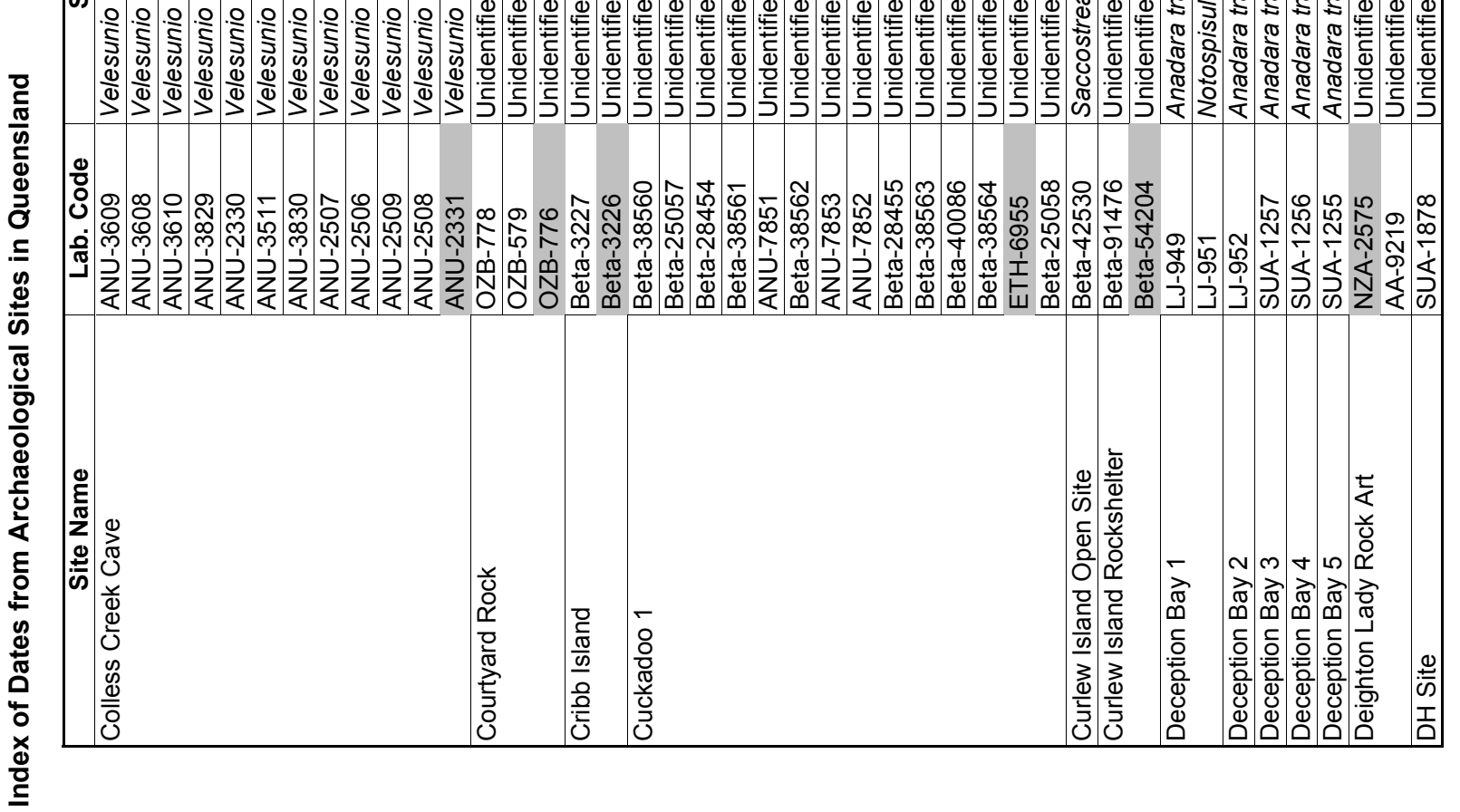

$\stackrel{2}{7}$

iे

8

8 


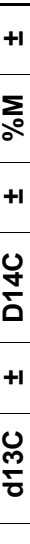

ก. 0.0 .5 .0 .0 .6 - 00000-

¿

พฺ

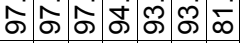

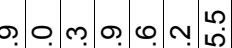

$\frac{0}{\mathfrak{Z}}$ 0 0 の 0 ம்

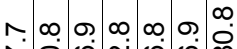
సิ่

N N N N N N

u্m

0000000

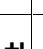

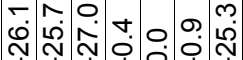

o

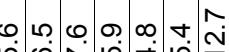

$\frac{\frac{1}{0}}{0}$ ல 0 ก ம்

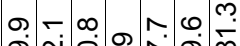

จุ่ สฺุ

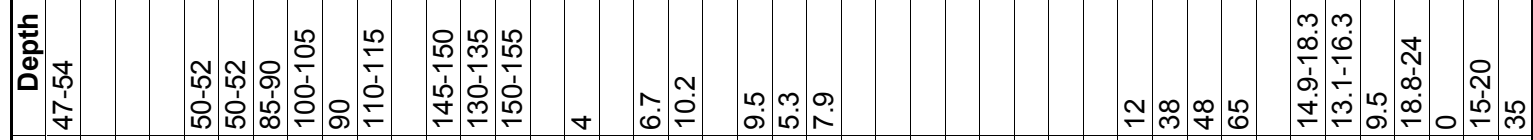

$\vec{x}$

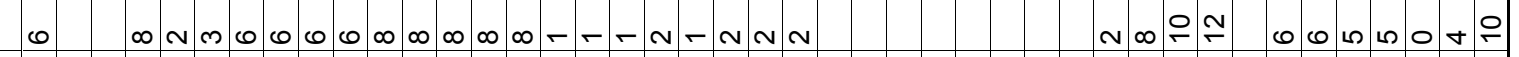

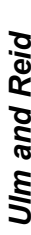

$\frac{0}{\frac{3}{0}}$

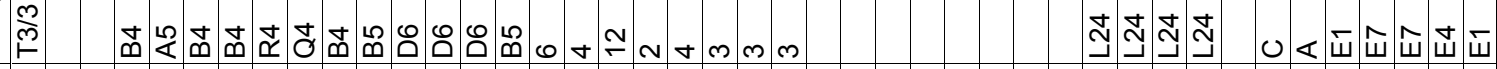

క

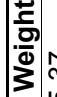

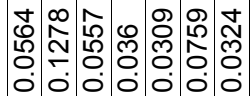

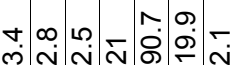

(2)

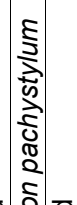

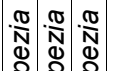

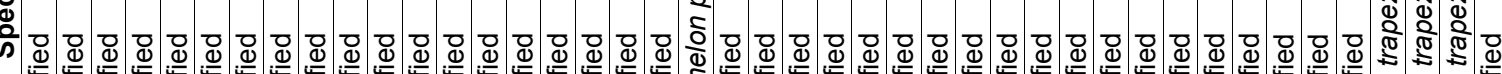

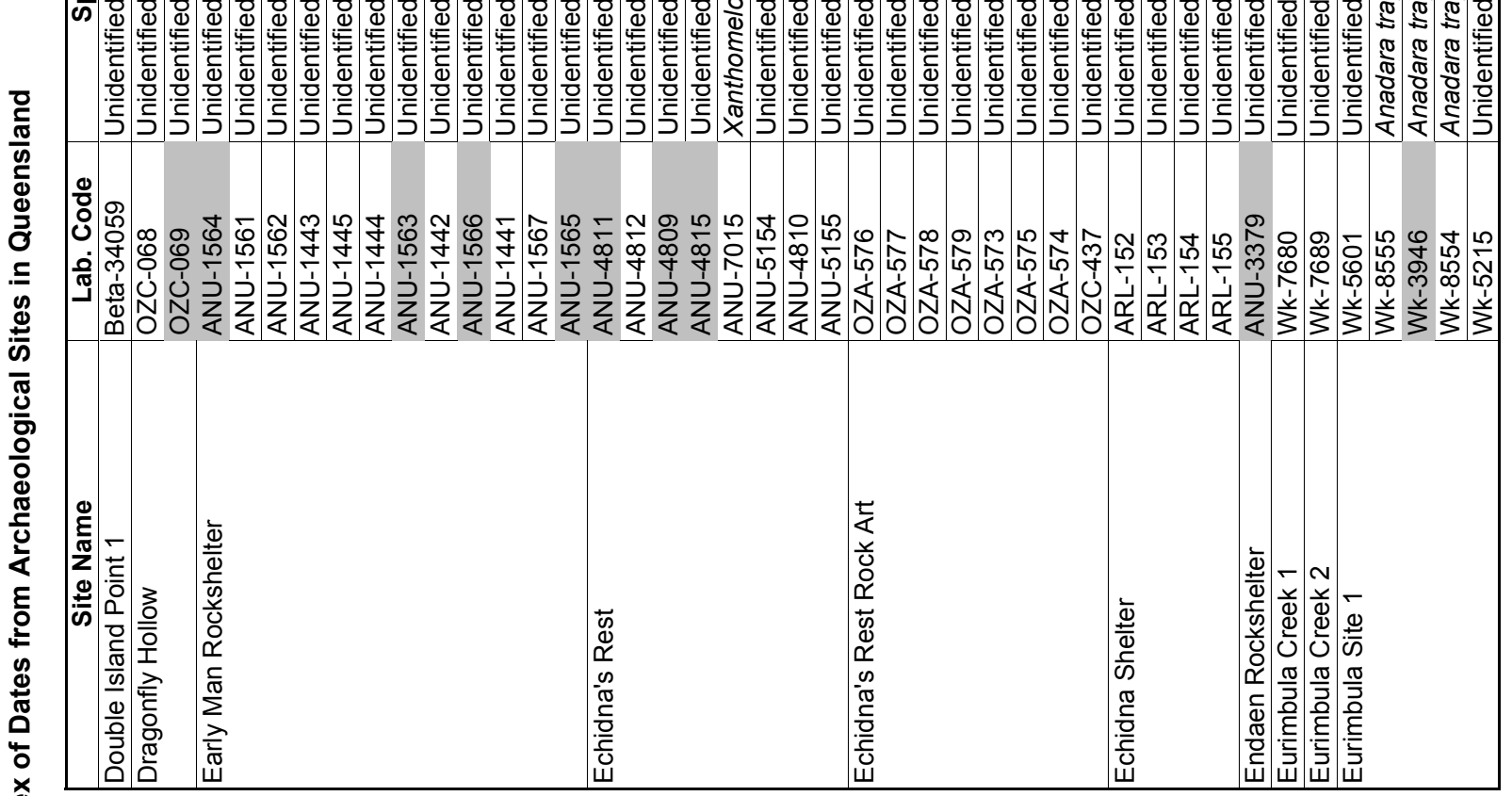

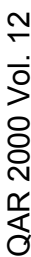




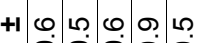

$\Sigma-1$

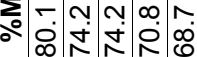

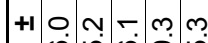

u 00.00

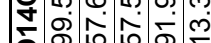

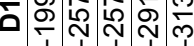

+ 1 ก

u

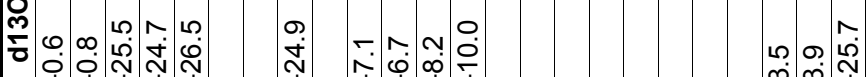

$\begin{array}{llllllllllllll} & 1 & 1 & 1 & & \end{array}$

+1 -

บ

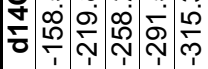

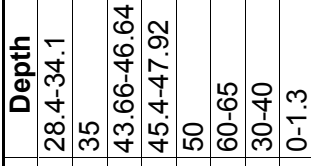

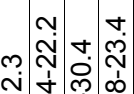

mై

구요

กั่

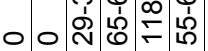
신

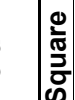

ชิ (1)

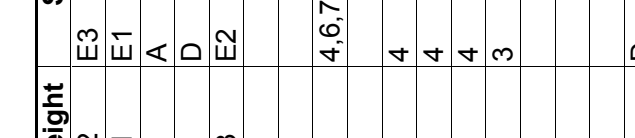

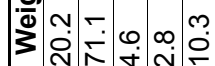

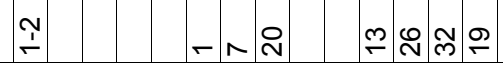

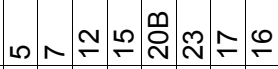

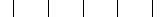

हิ)

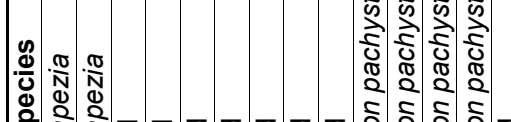

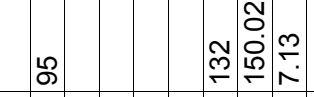

ப)

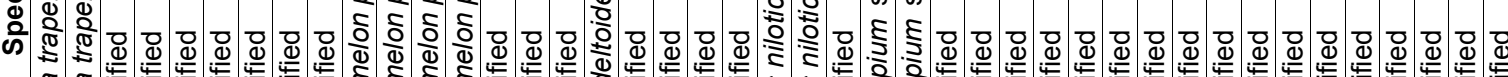

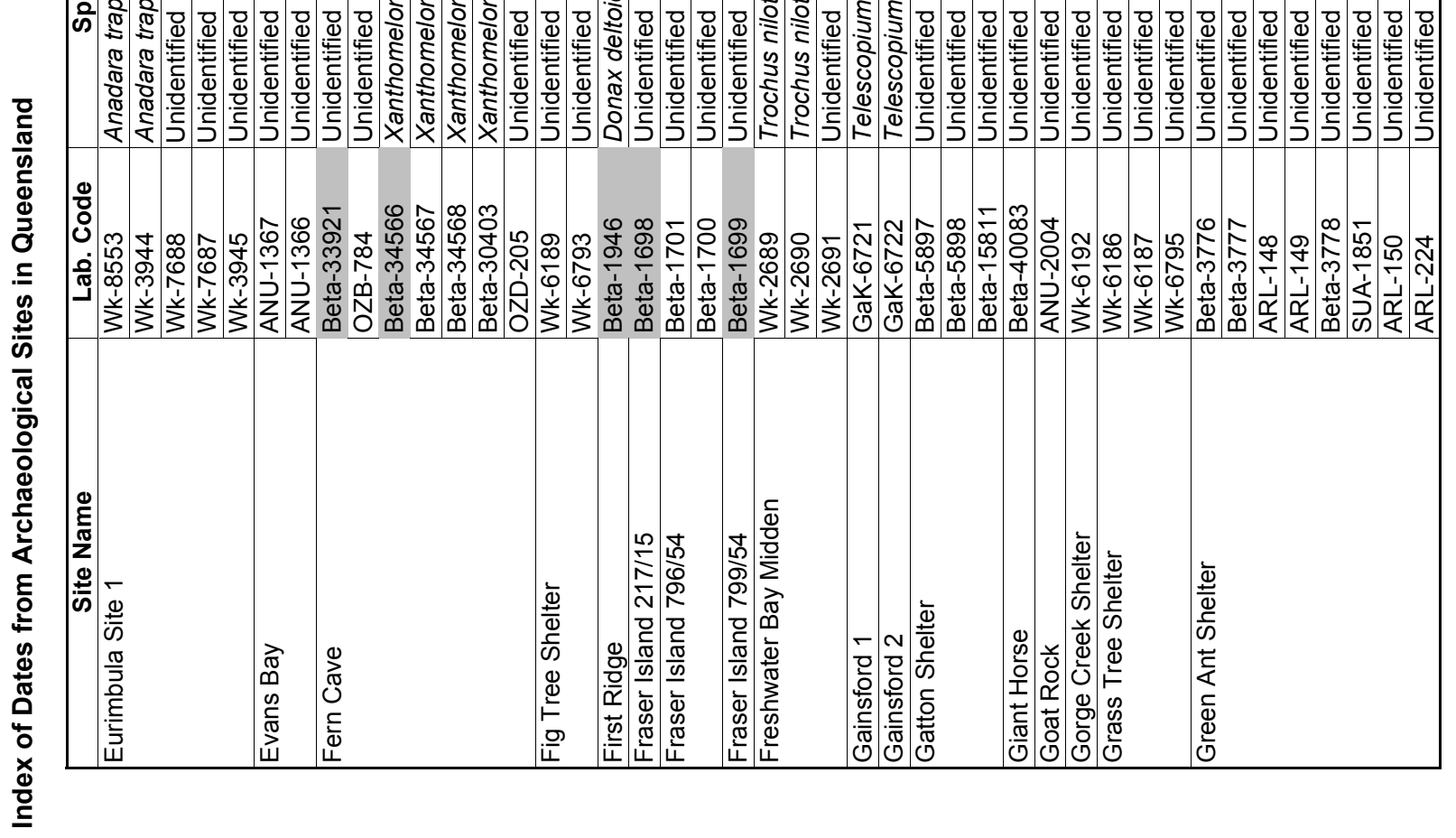

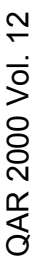




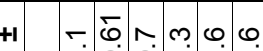

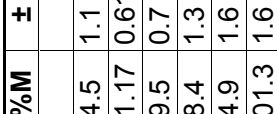

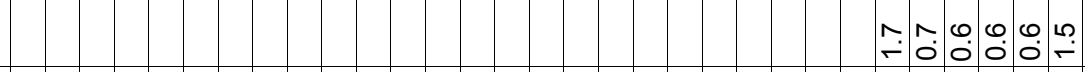

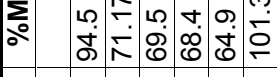

ค ब m m 0

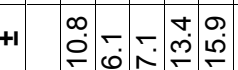

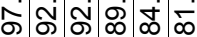

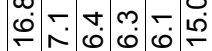

Z

à

-

+1 ( 12

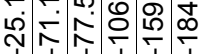

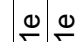

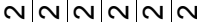

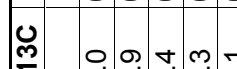

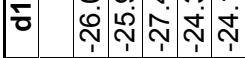

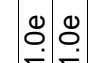

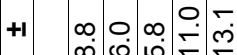

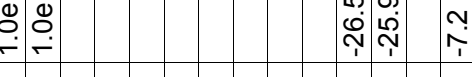

0.000 .0

热

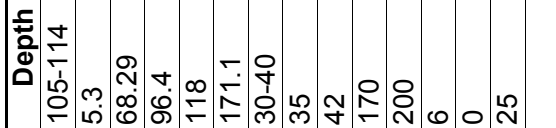

¿.

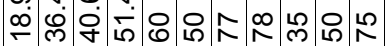

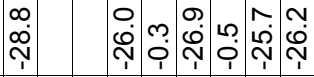

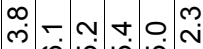

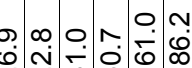

$\vec{x}$

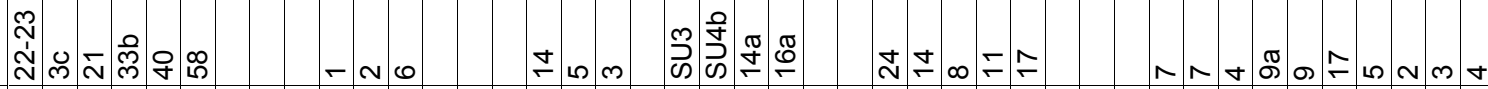

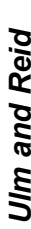

$\frac{100}{\frac{3}{0}}$

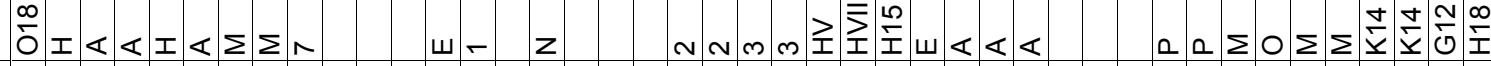

క

童

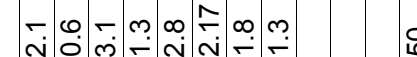

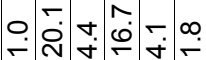

$\frac{\mathscr{\varpi}}{\mathscr{\Phi}}$

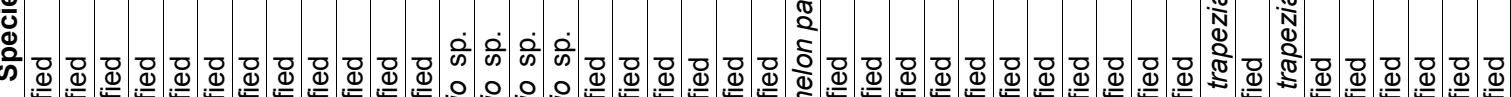

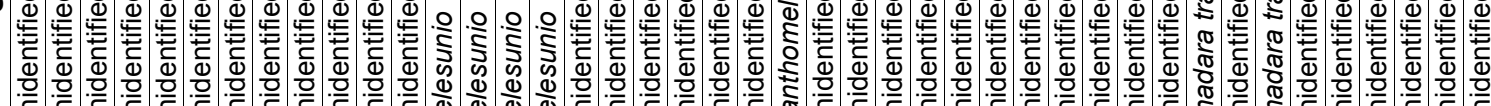

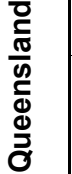

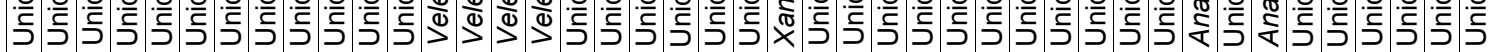

ن.

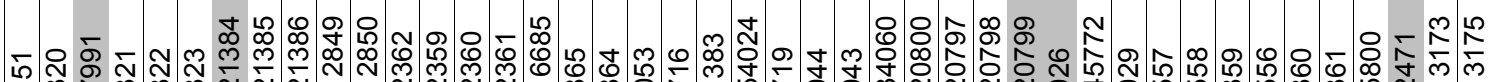
T)

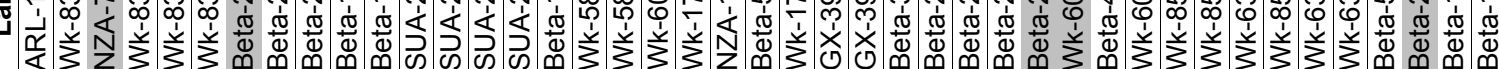

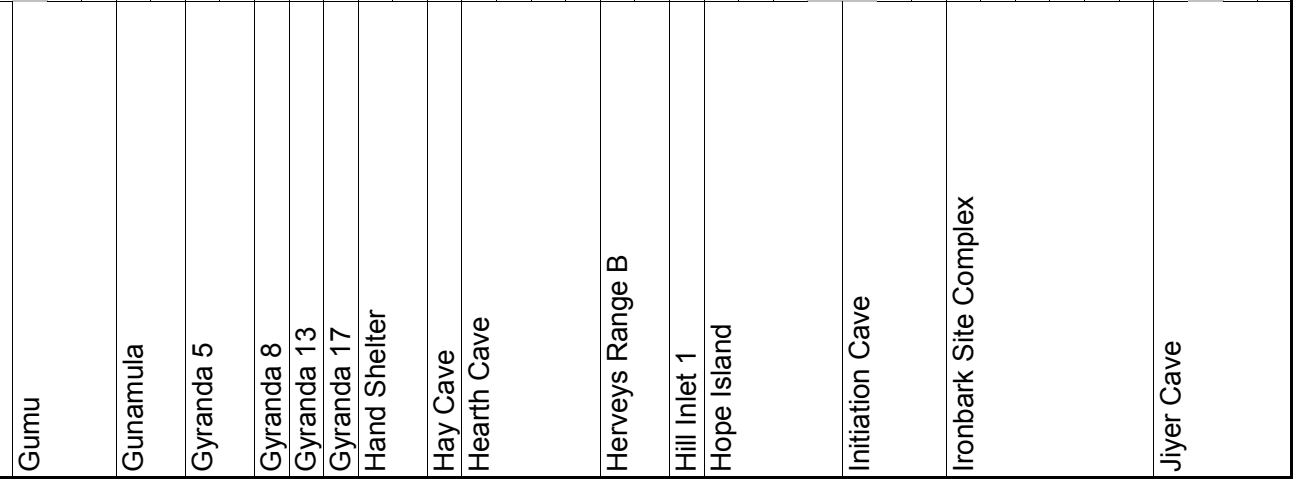




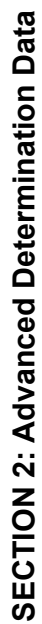

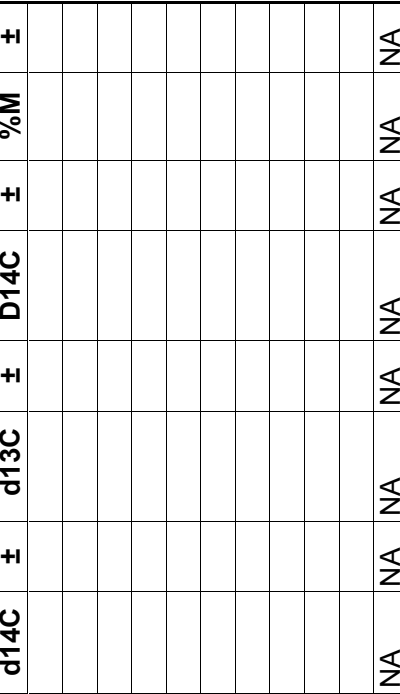

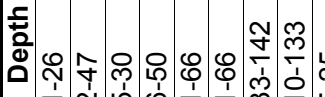

$\overleftarrow{z}$

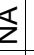

$\Sigma$

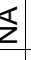

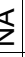

$\varangle$

$\mathbb{z}$

$\mathbb{Z}$

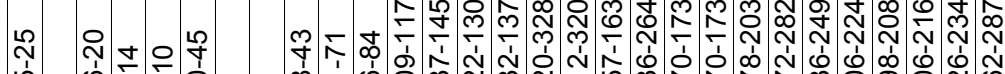

m

$\vec{x}$

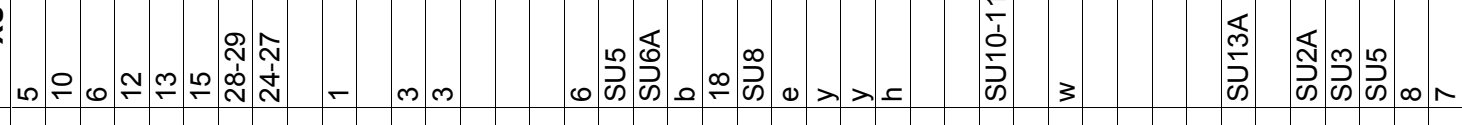

:

กิ

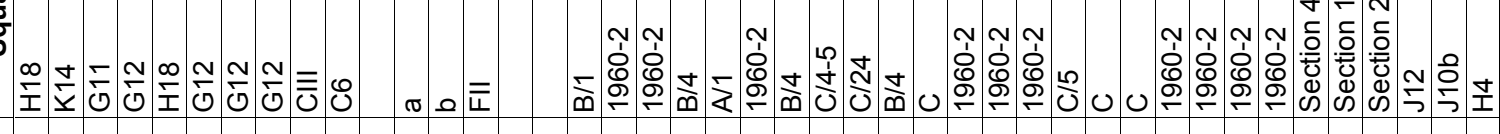

蒙

요

is

$\frac{\mathscr{\omega}}{\grave{\omega}}$

象

$\frac{\pi}{N}$

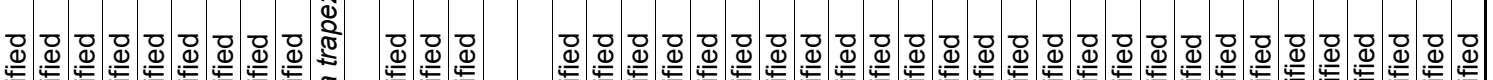

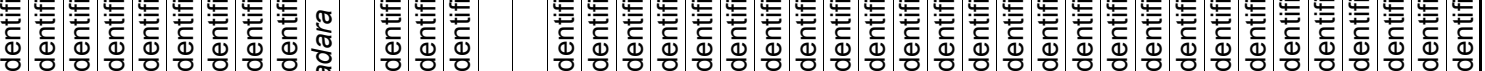

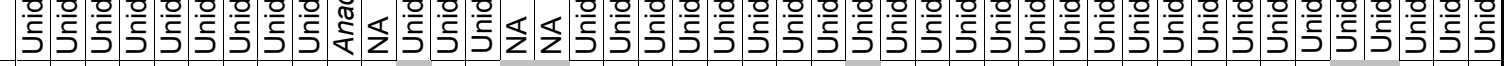

\%

己.

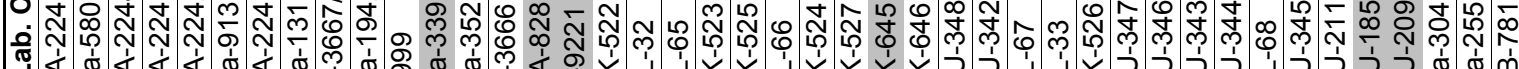

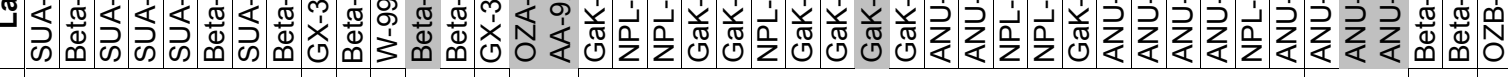

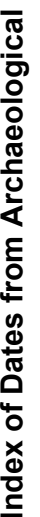

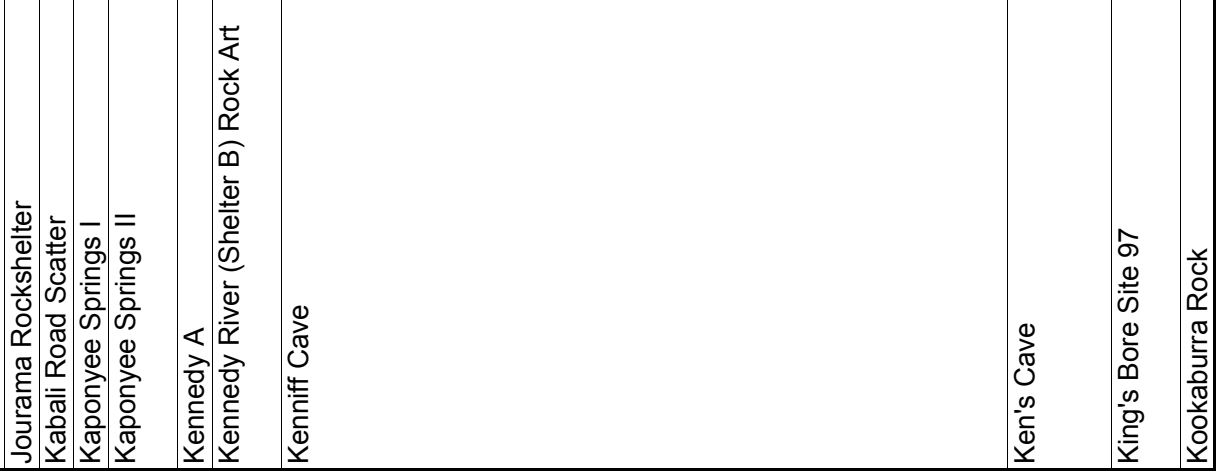

 ㅎํ

\& 


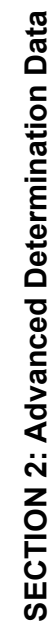

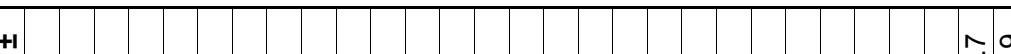

$\sum_{0}^{5}$

సٓن

$\frac{a}{2}$

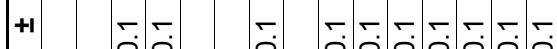

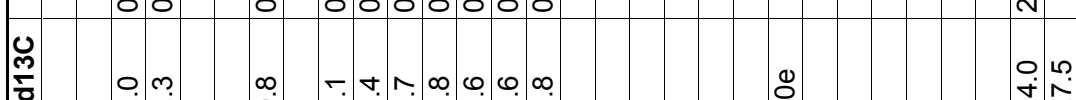

$+1$

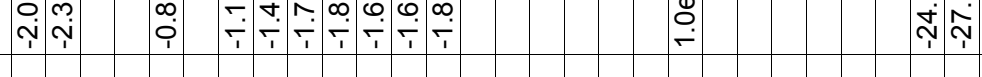

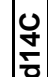

$\frac{\mathbf{a}}{\mathbf{a}}$

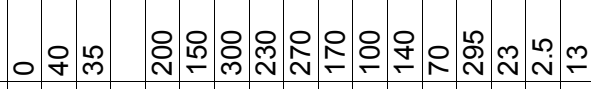

음

ㄴ.

L

กิ่

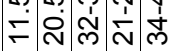

$\vec{x}$

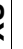

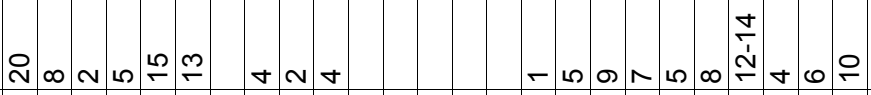

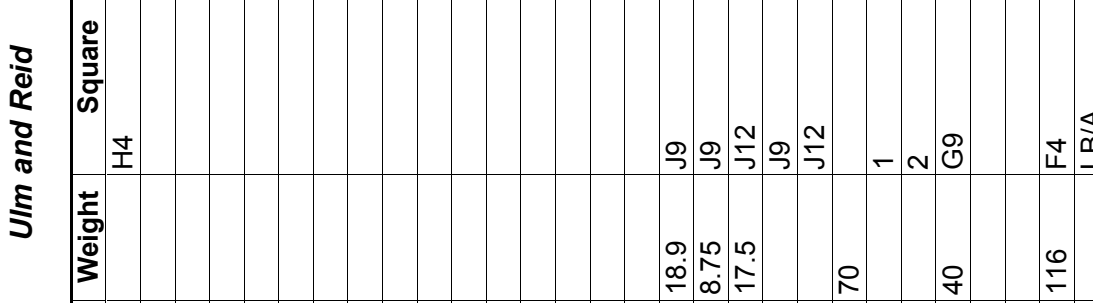

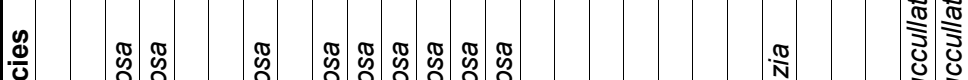

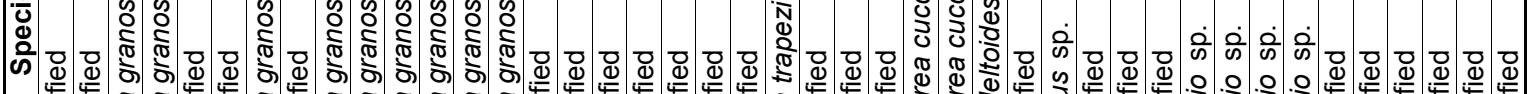

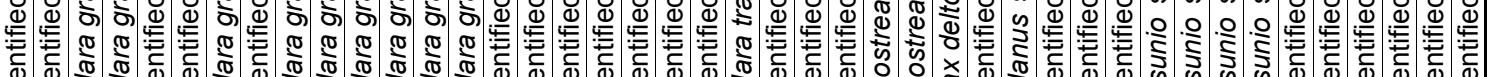

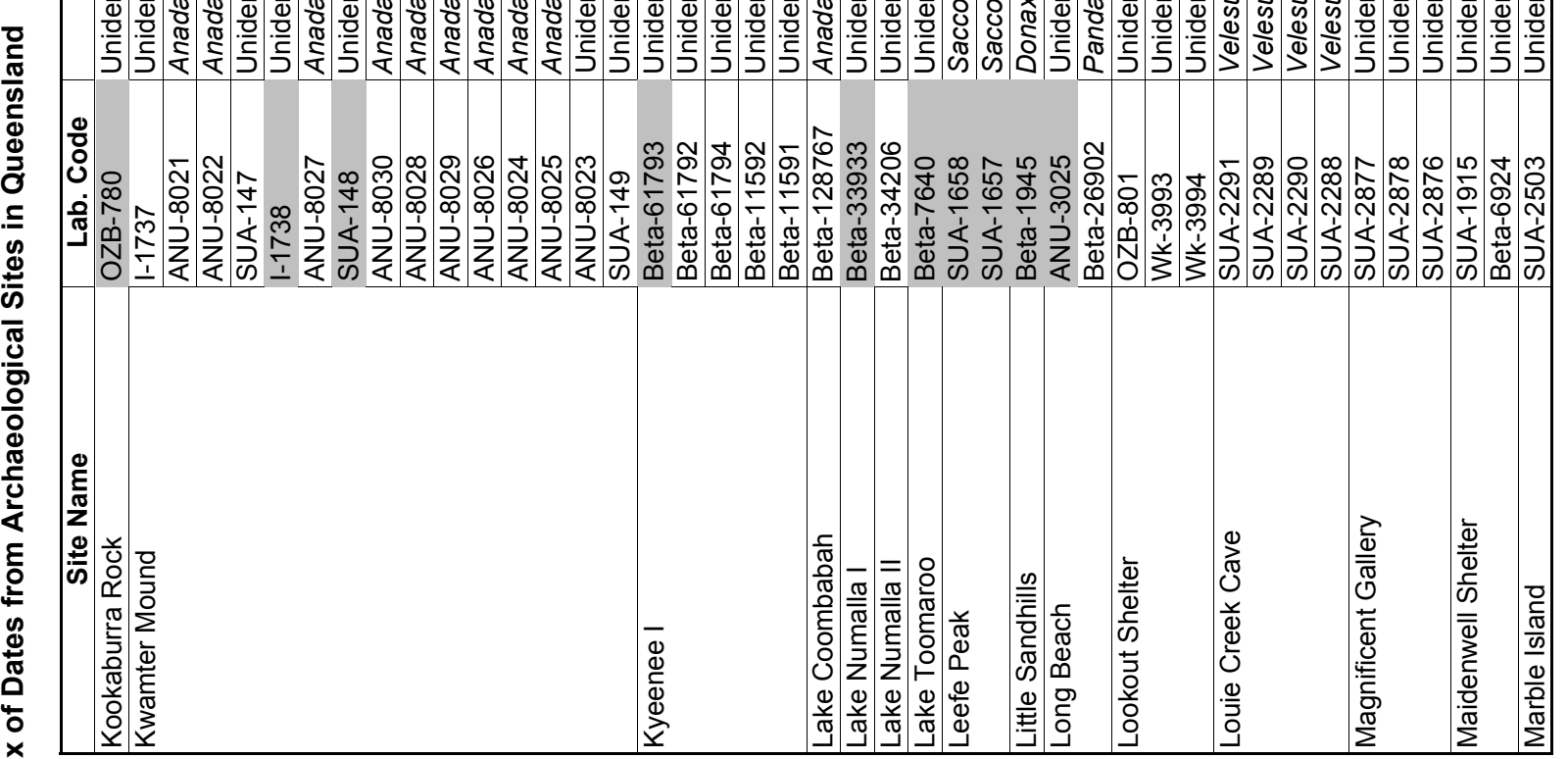

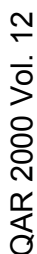




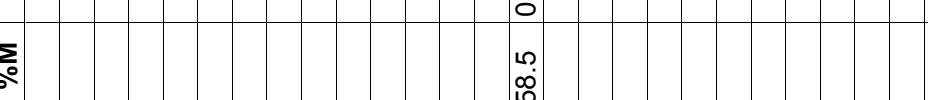

$\infty$
$\infty$
$\infty$
$\infty$ m 00 0 0 N

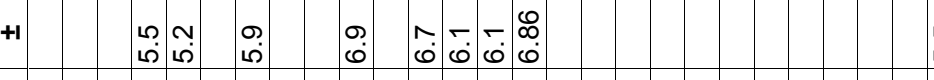

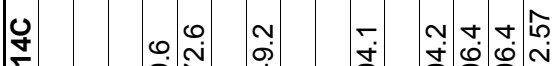
$\bar{a}$

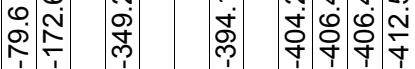

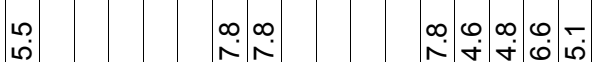

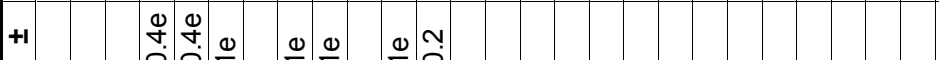
遂

$00 \div \div 0$

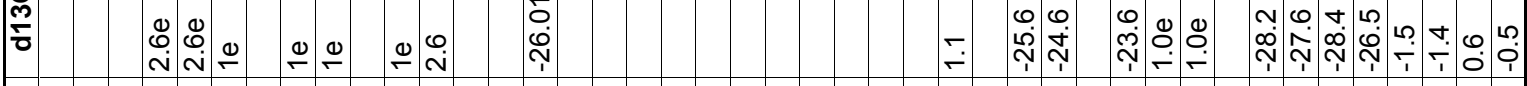

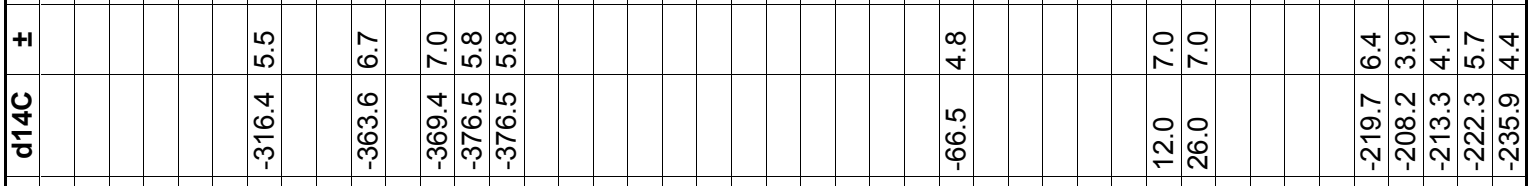

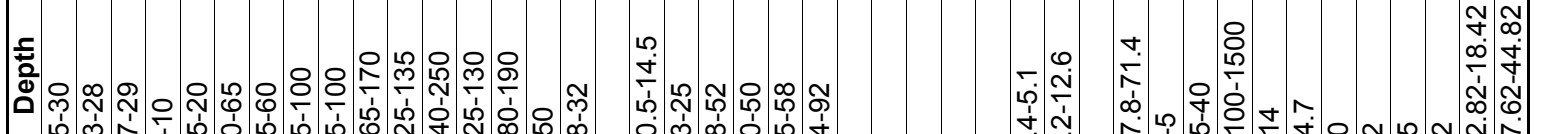

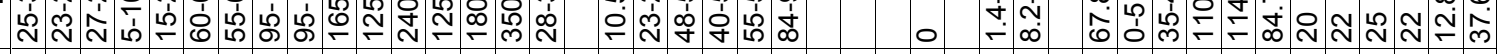

$\vec{x}$

¿

onn-4

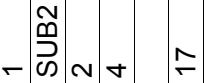

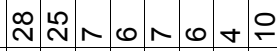

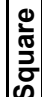

(2)

క

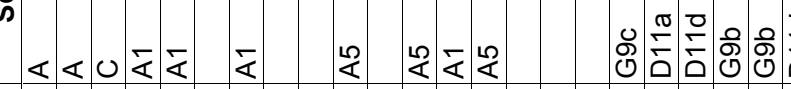

농

3̇oi

悹

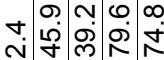

\section{$\widehat{\mathscr{d}}$}

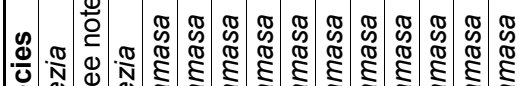

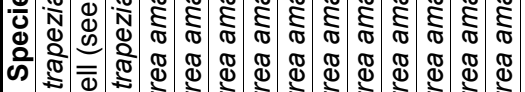

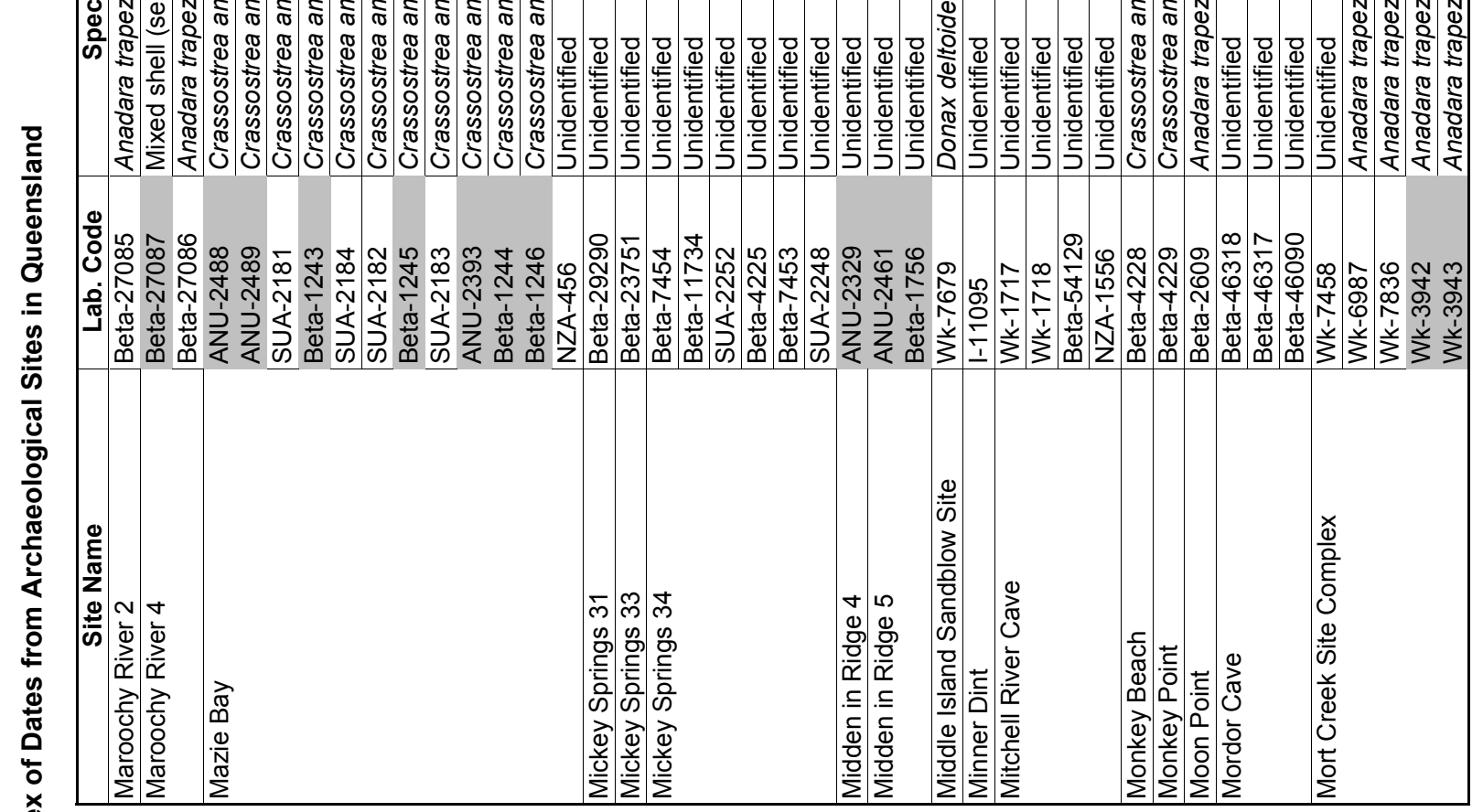
$\varangle \infty m m m \ll<$

으으요 ₹ . 


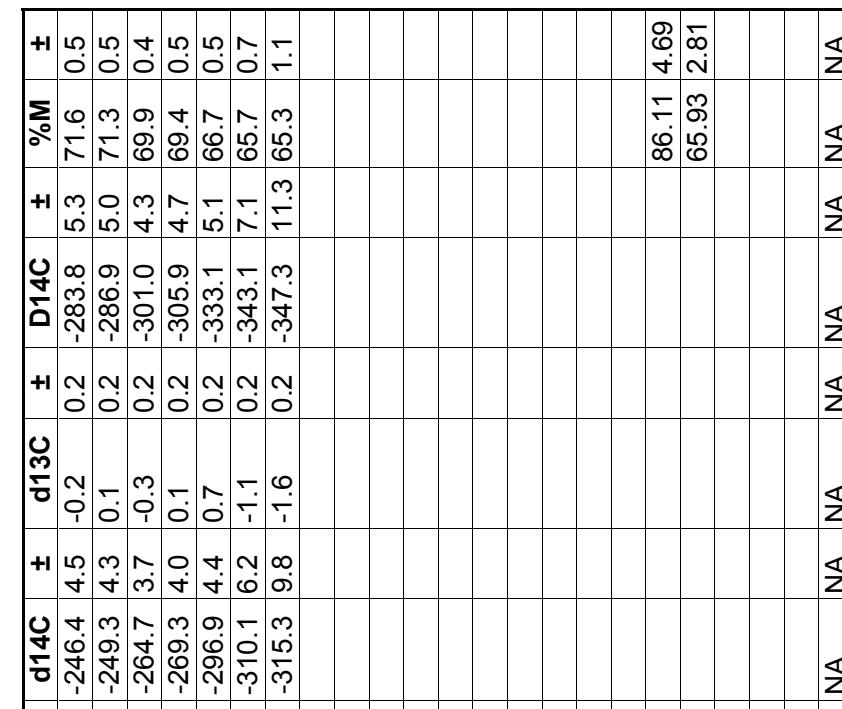

$\mathbb{z} \mathbb{z} \quad$ z $\mathbb{z} \frac{\mathbb{z}}{z} \mathbb{z}$
$\$ \mathbb{Z} \quad \mathbb{z} \mathbb{z} \mathbb{z}$

$\frac{\mathbb{z}}{z} \quad \mathbb{z} \mathbb{z} \frac{\mathbb{z}}{z} \frac{\mathbb{z}}{z}$

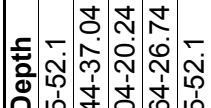

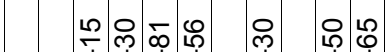

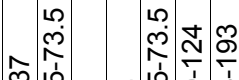
$\mathbb{z} \frac{\mathbb{z}}{z} \quad \mathbb{z} \mathbb{z} \frac{\mathbb{z}}{z} \frac{\mathbb{z}}{z}$

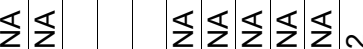

$\mathbb{z} \mathbb{z} \quad \mathbb{z} \mathbb{z} \frac{\mathbb{z}}{z} \frac{\mathbb{z}}{\mathbf{t}}$

$\mathbb{z} \mathbb{z} \quad \mathbb{z} \mathbb{z} \mathbb{z} \frac{\mathbb{z}}{z}$

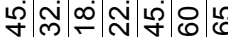

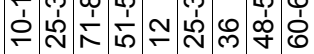

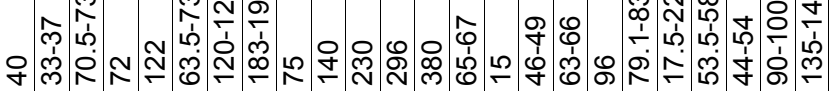

$\vec{x}$

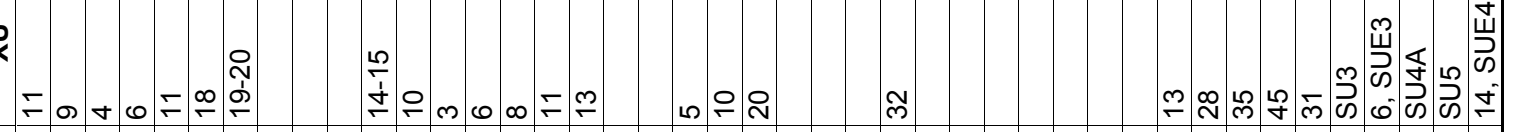

离

के

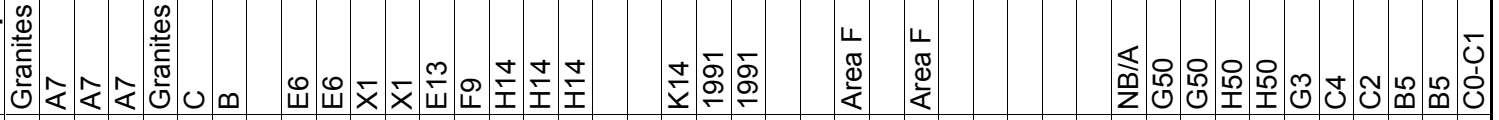

$\frac{\pi}{\frac{0}{2}}$

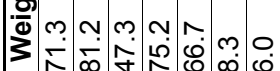

으

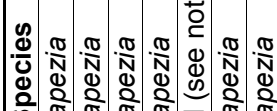

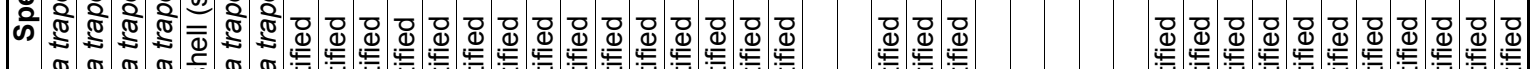

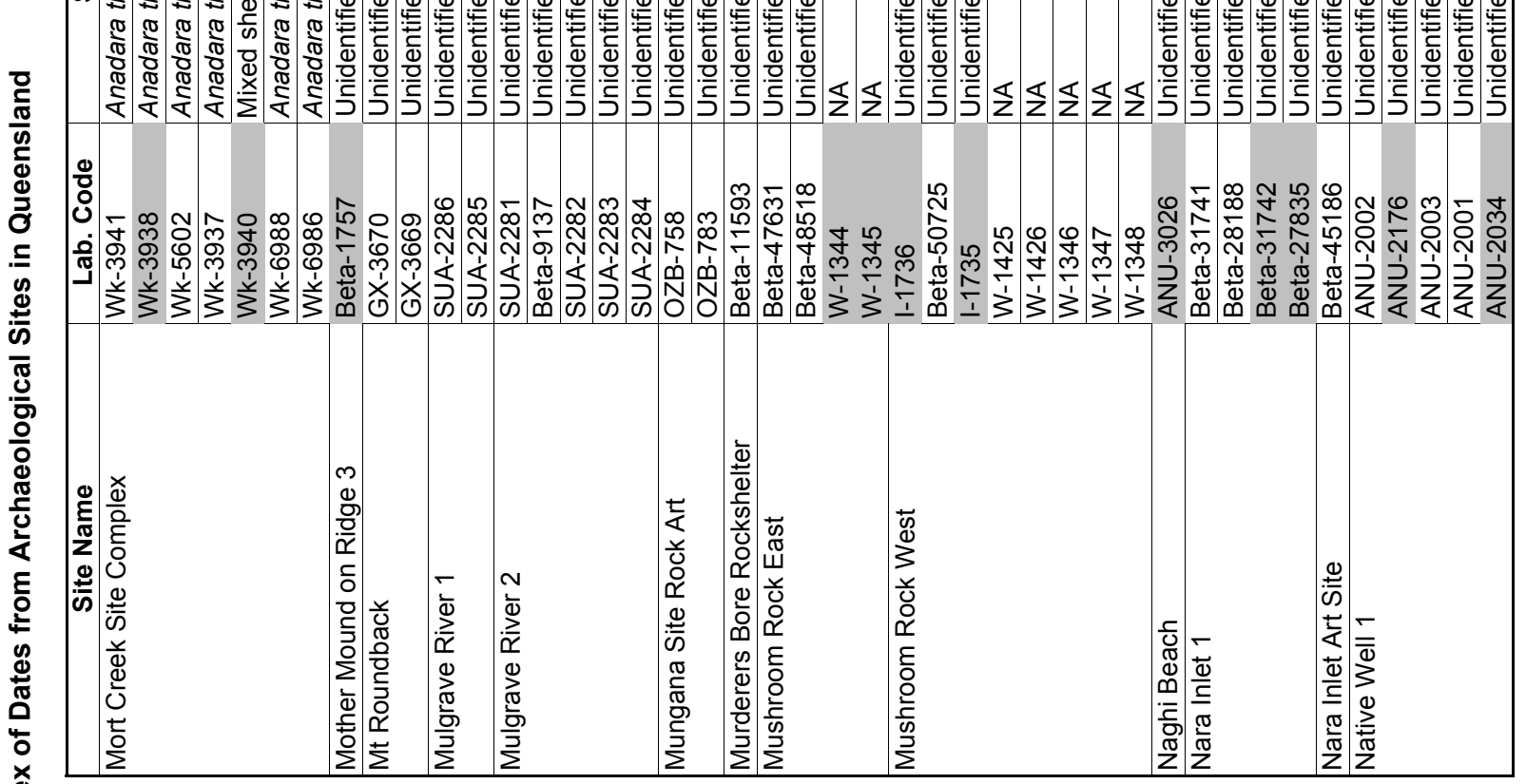




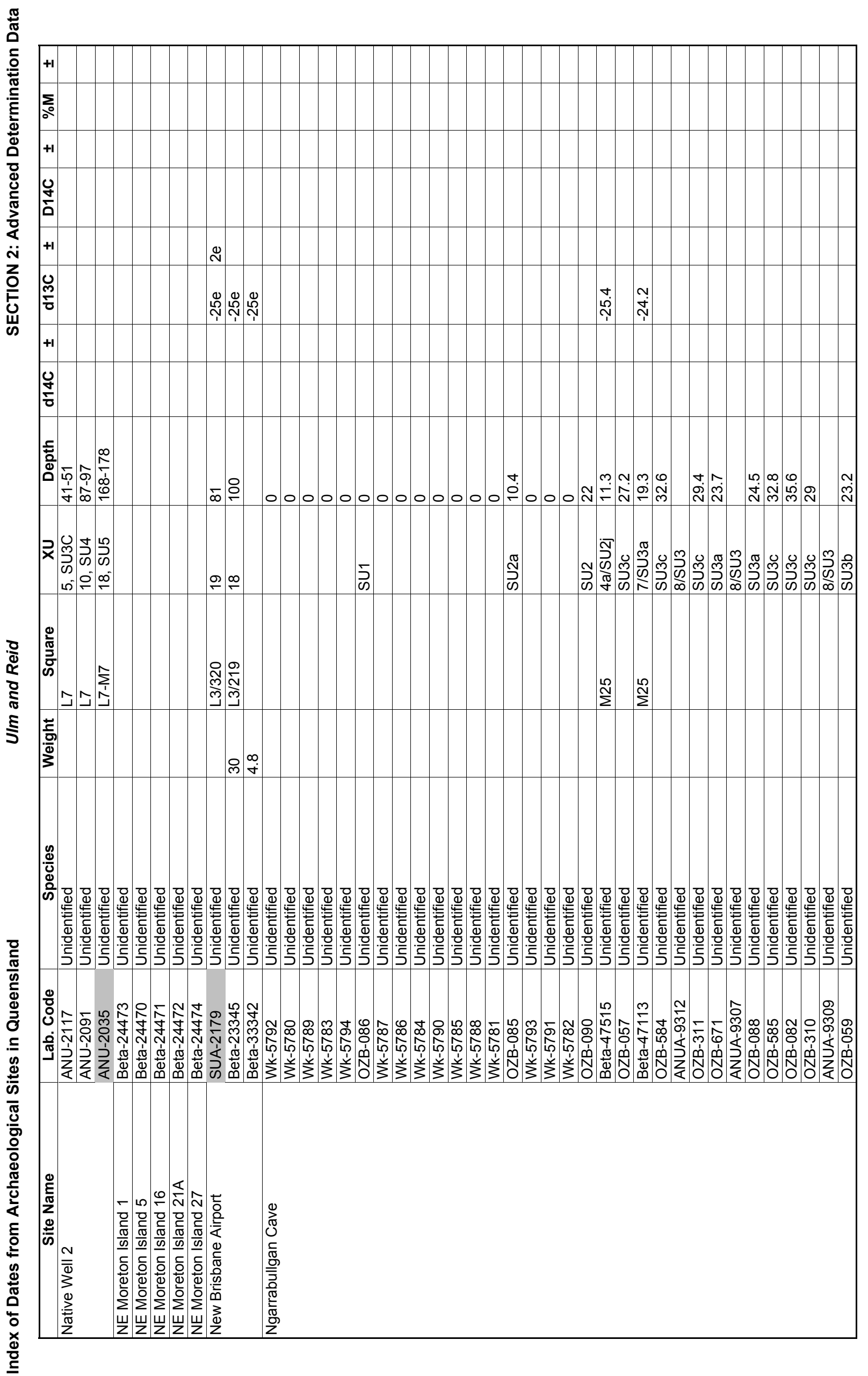




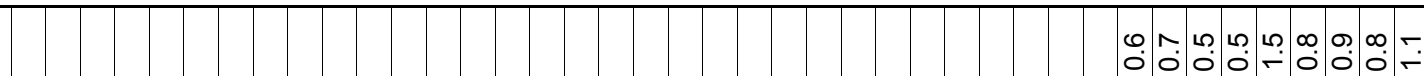
$\sum_{\circ}^{\infty}$

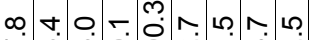
ஸ் $+1$

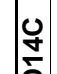

$+2$

$\frac{0}{\frac{m}{0}}$

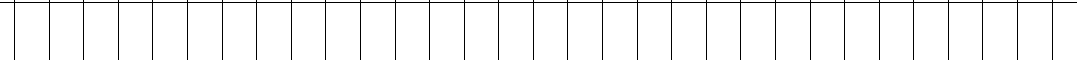

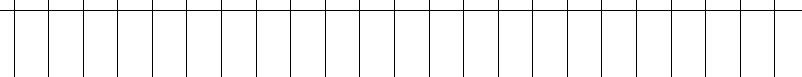

$\frac{0}{0}$

6.

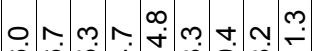

咅

章

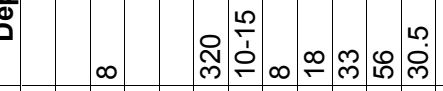

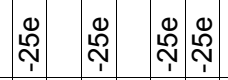

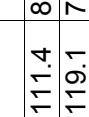

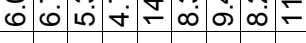

$\vec{x}$

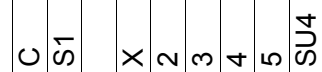

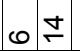

ก กิำกำ

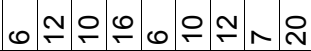

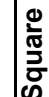

б̄o

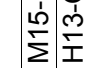

$\stackrel{\sim}{\sim}$

స்

$\stackrel{\infty}{\sim}$

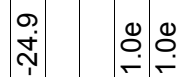

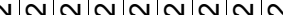
000000000

พุำ

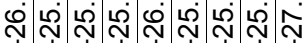

సิن

ه

بิ

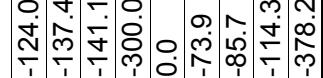

蒿

$\sum \sum I$

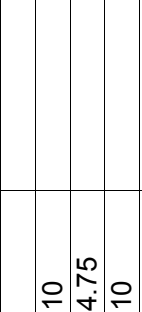

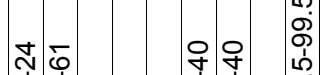

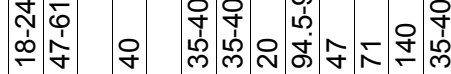

ก)

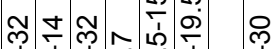

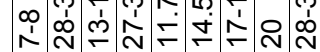

.

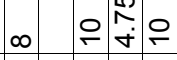

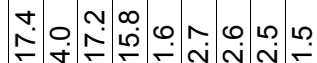

\section{$\frac{\mathscr{\Xi}}{\mathcal{\Xi}}$}

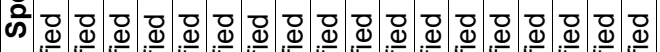

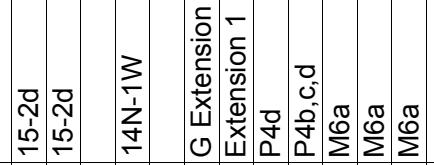

$\infty \ll \infty \emptyset \ll \ll \ll \omega \ll$

రำ

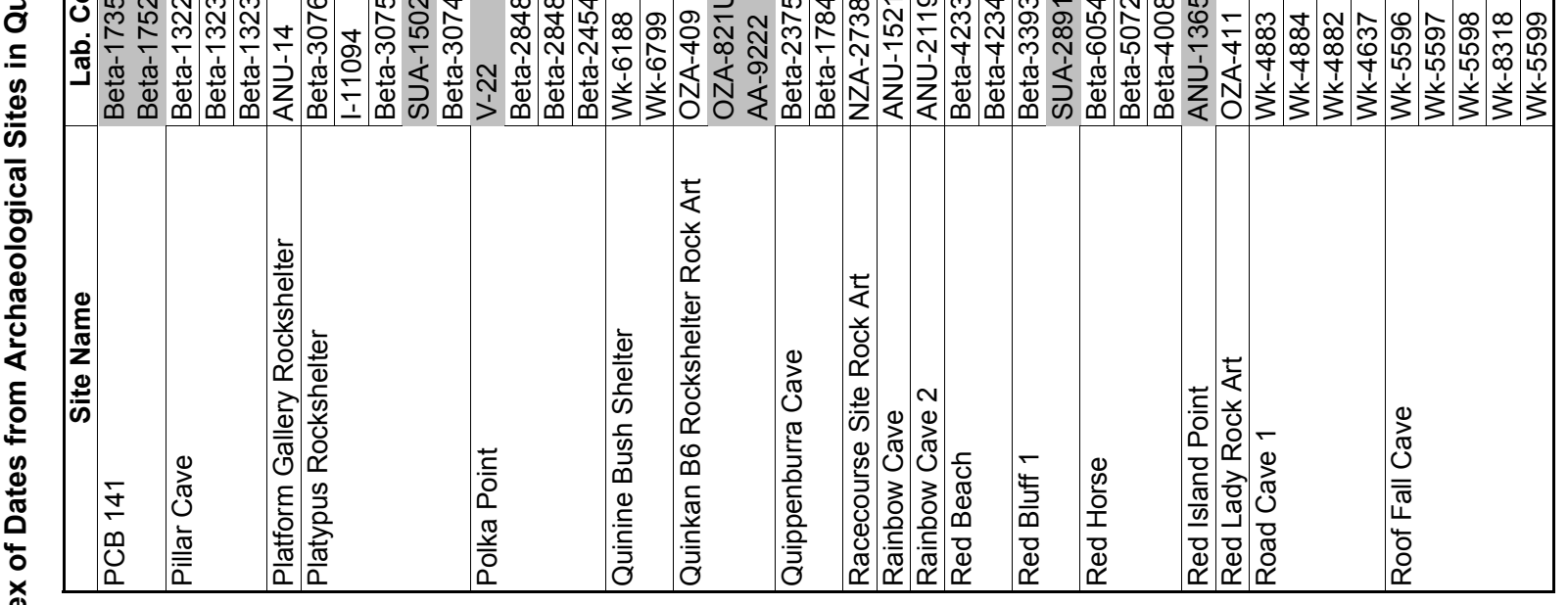




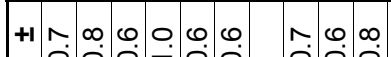

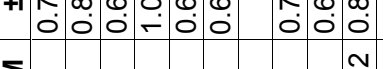

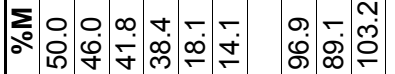

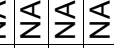

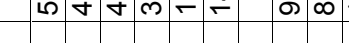

$\mathbb{z} \mathbb{z} \mathbb{Z}$

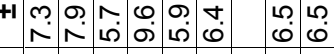

$\mathbb{z} \mathbb{z} \mathbb{z} \mathbb{z}$

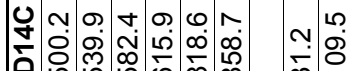

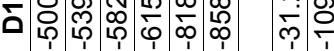

$\operatorname{tin} N$ N $N$ N

$\leqslant \leqslant \frac{1}{z}$

000000

$\mathbb{z} \mathbb{z} \mathbb{z}$

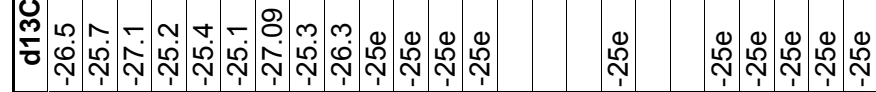

$+0.5 .50 .00$

$\mathbb{z} \mathbb{z} \frac{\mathbb{z}}{z}$

$\begin{array}{lllllll}1 & 0 & 0\end{array}$

$\mathbb{z} \mathbb{z} \mathbb{z}$

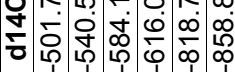

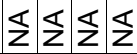

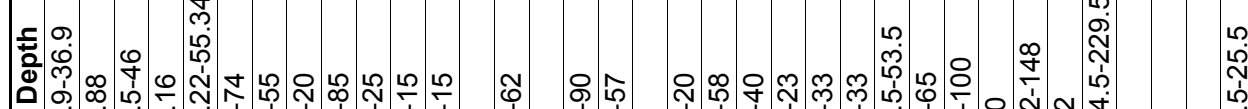

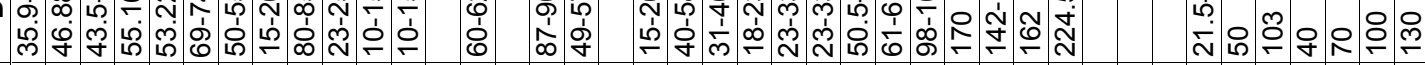

$\vec{x}$
$\stackrel{\infty}{\div}$ ลักส
ָิ
요 $=$

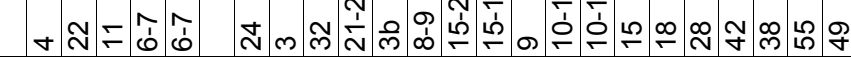
$0 \stackrel{2}{2}$

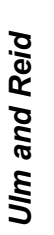

产

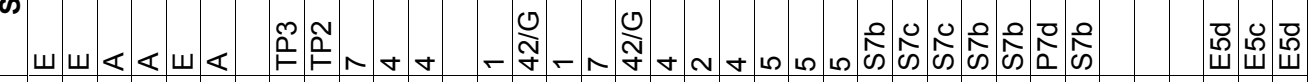

苔

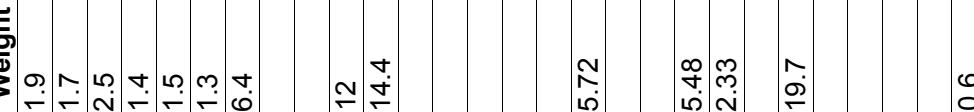

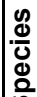

के

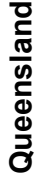

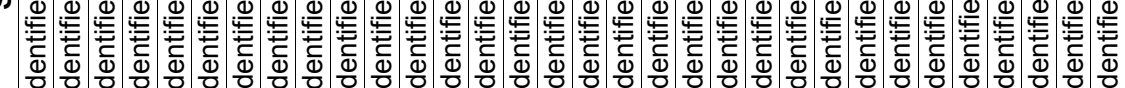

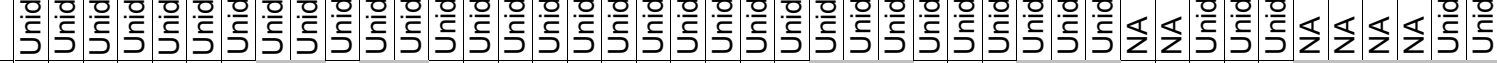

O.

ن.

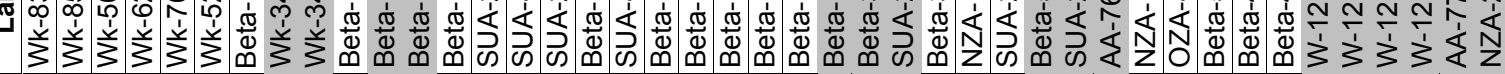

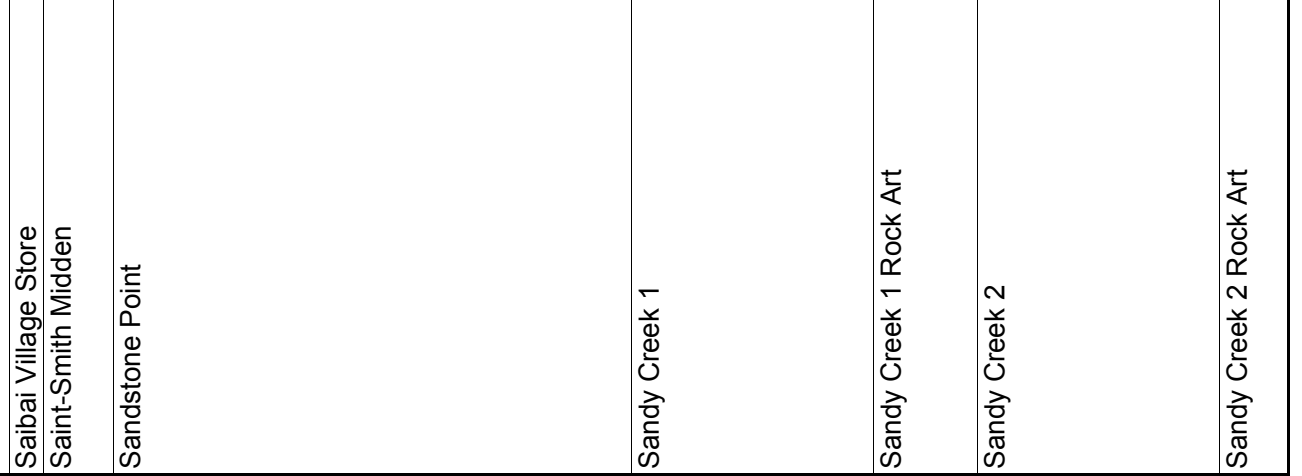


+

$\sum$ n?

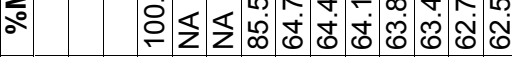

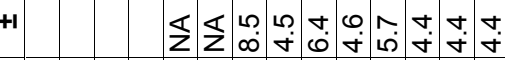

y तmnmए

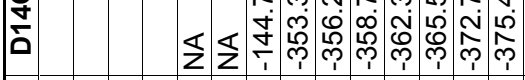

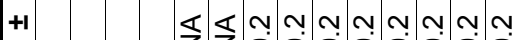

10.0 .0 .00

倠

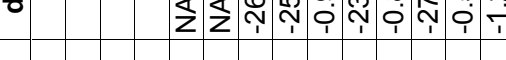

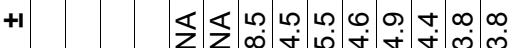

-

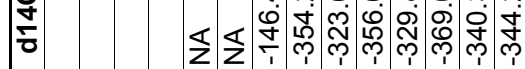

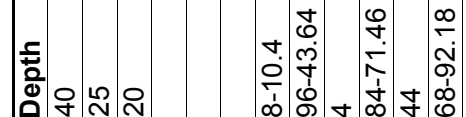

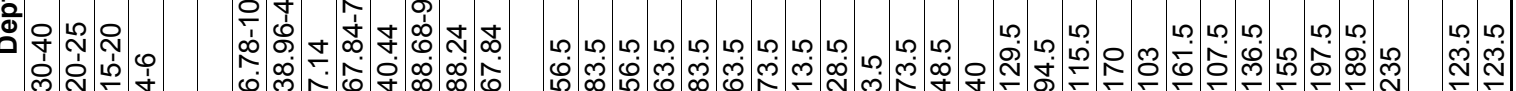

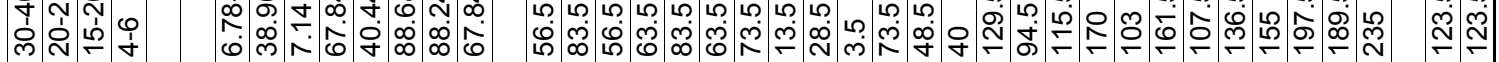

$\vec{x}$

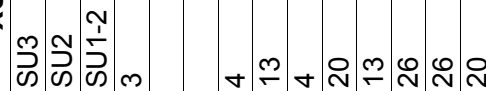

葛

ะ

ส

క

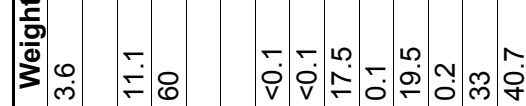

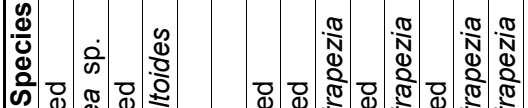

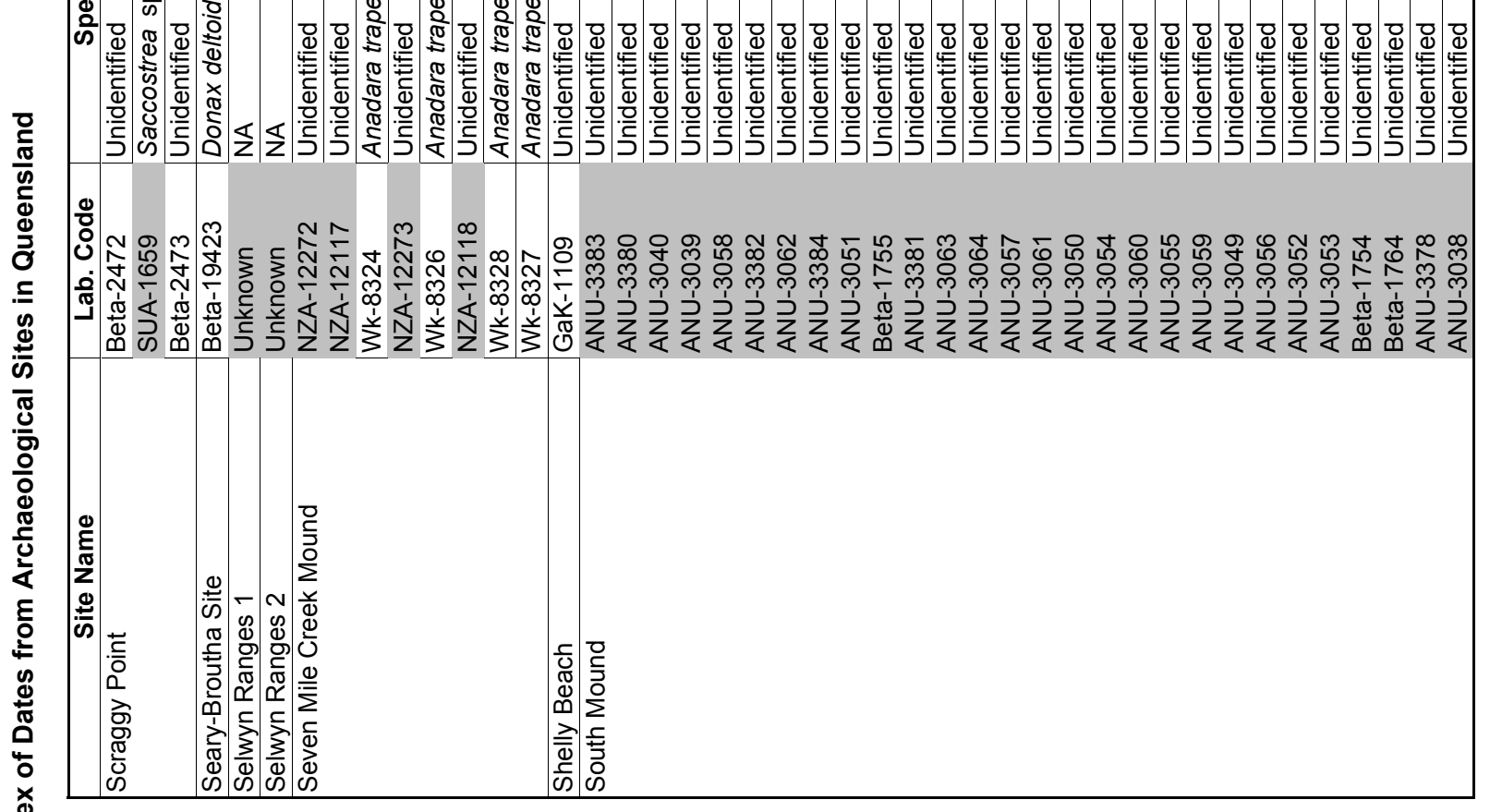




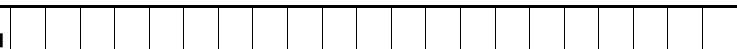

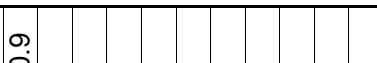

0 ○.

ம

$\sum_{0}$

우

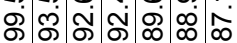

$+1$

$\underset{0}{+}$

\begin{tabular}{l|l|l|l}
$\infty$ \\
0 \\
0
\end{tabular}

$N \infty$

U 8 -

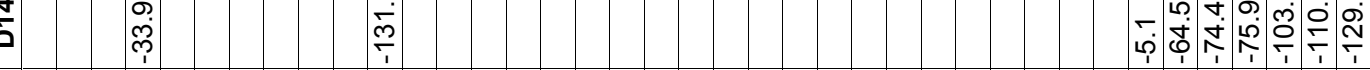

$+1$

๑.

- 100000

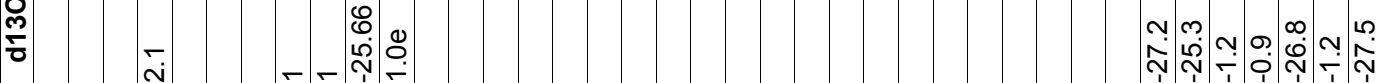

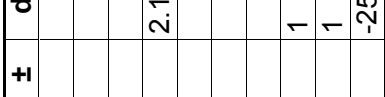

$\infty$ の の m 0 ட

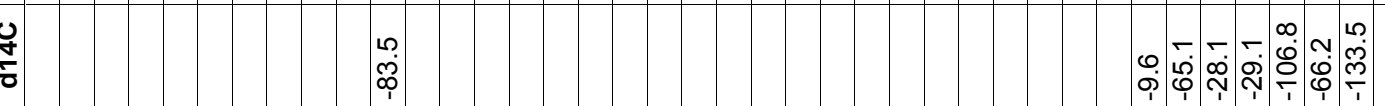

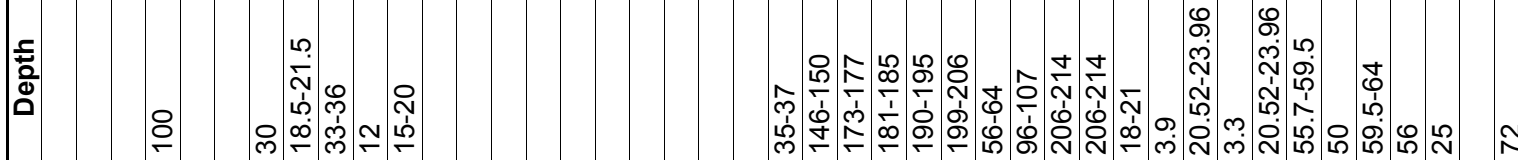

$\vec{x}$

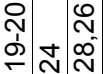

r

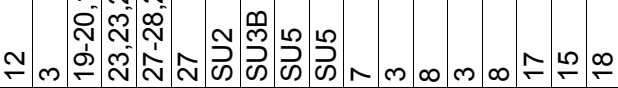

$\infty$ 의 $\stackrel{2}{\div}+$

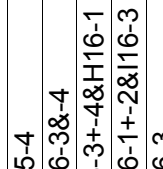

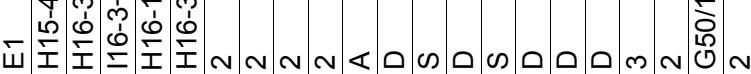

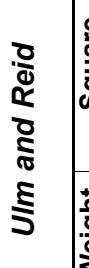

ติ

t⿳亠二口犬

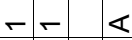

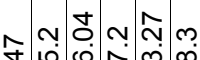

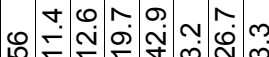

กิ

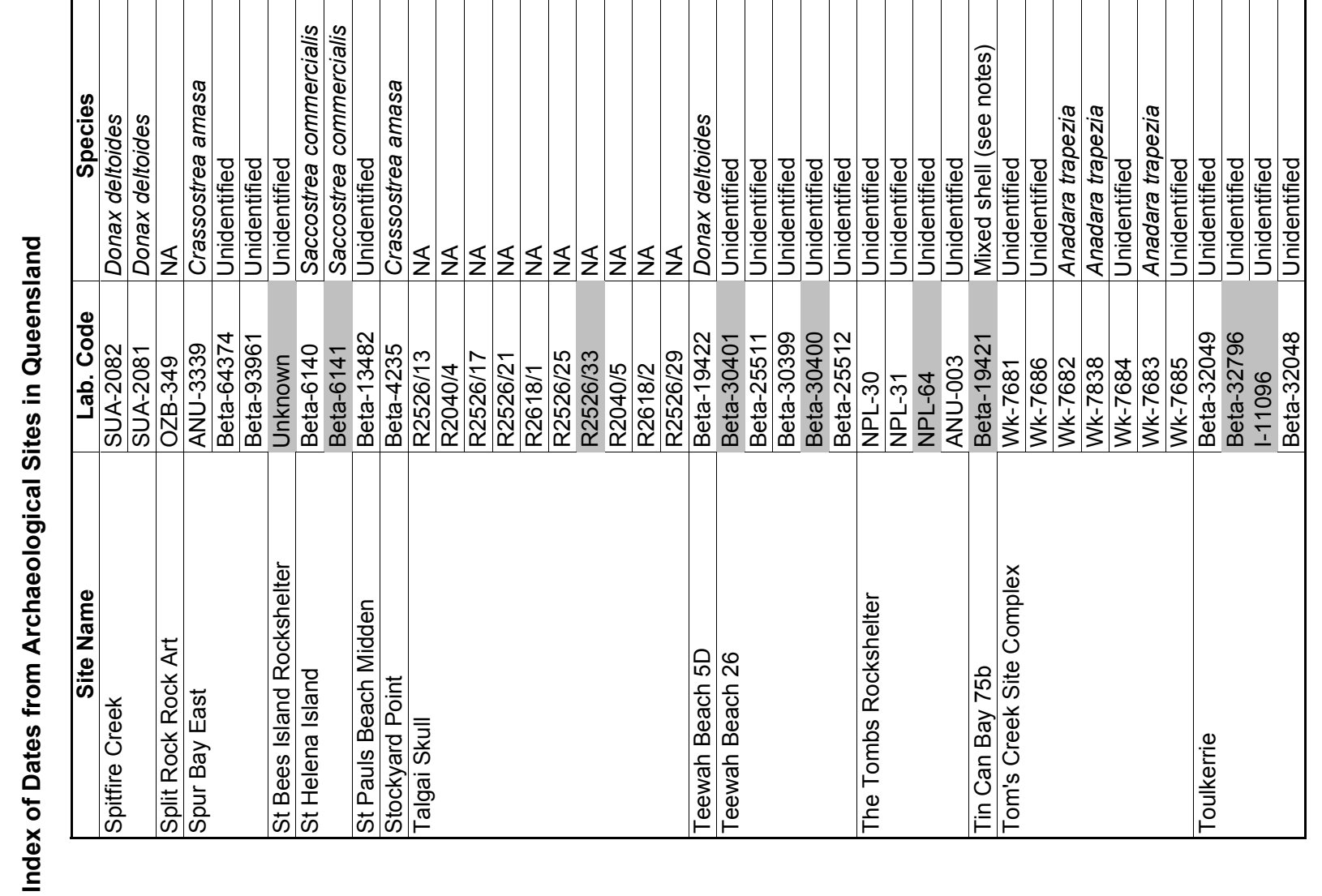




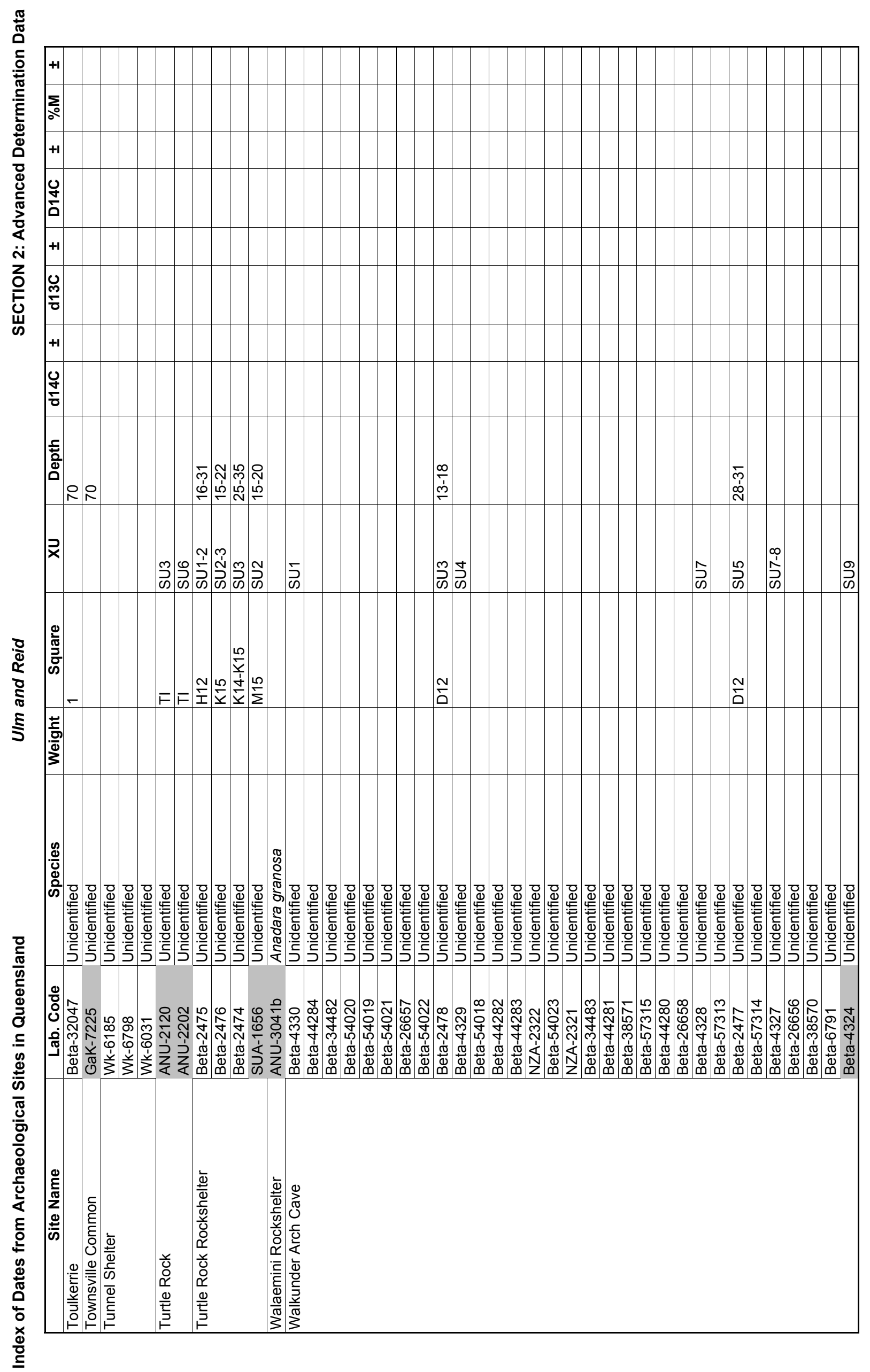




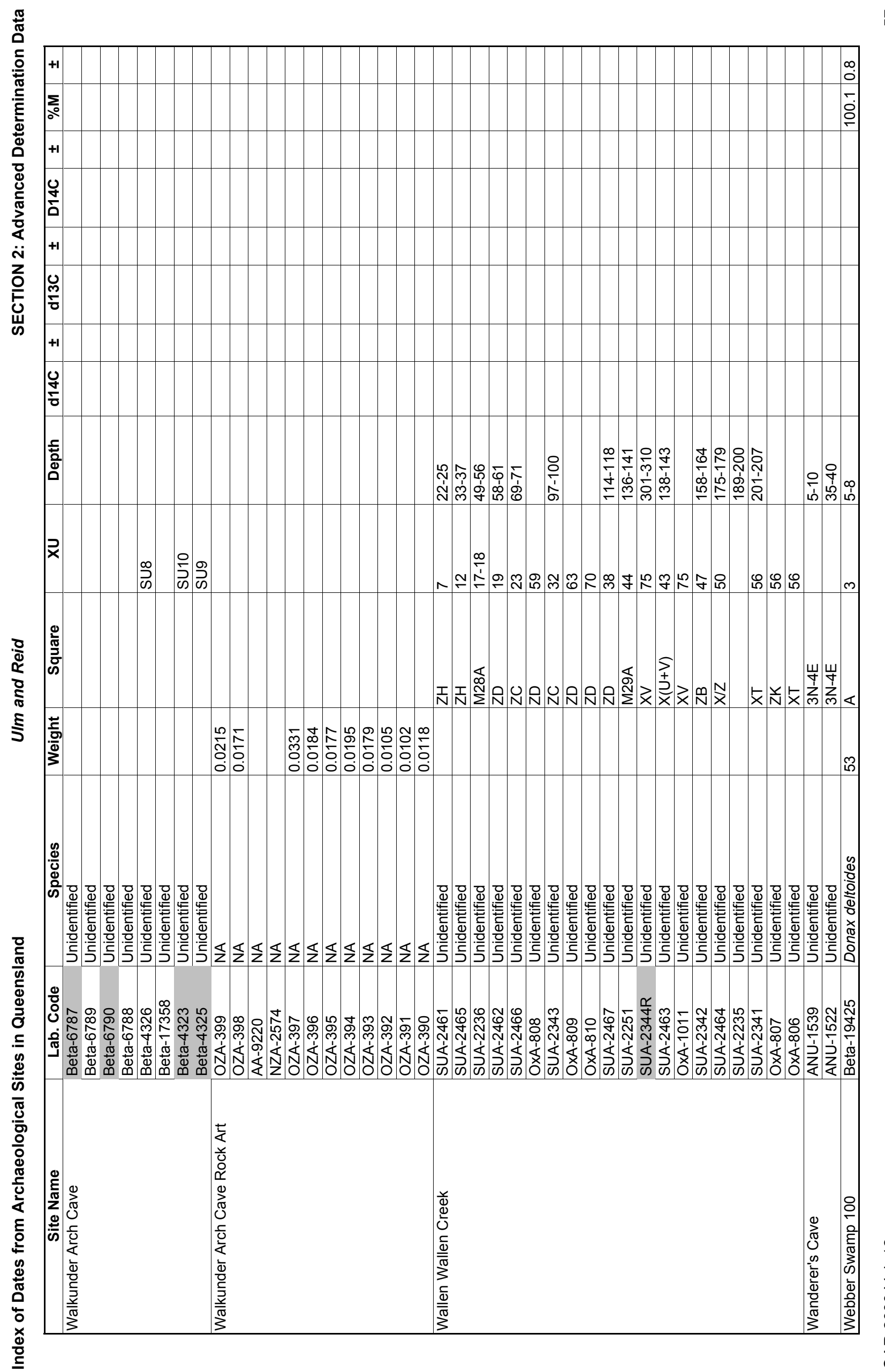




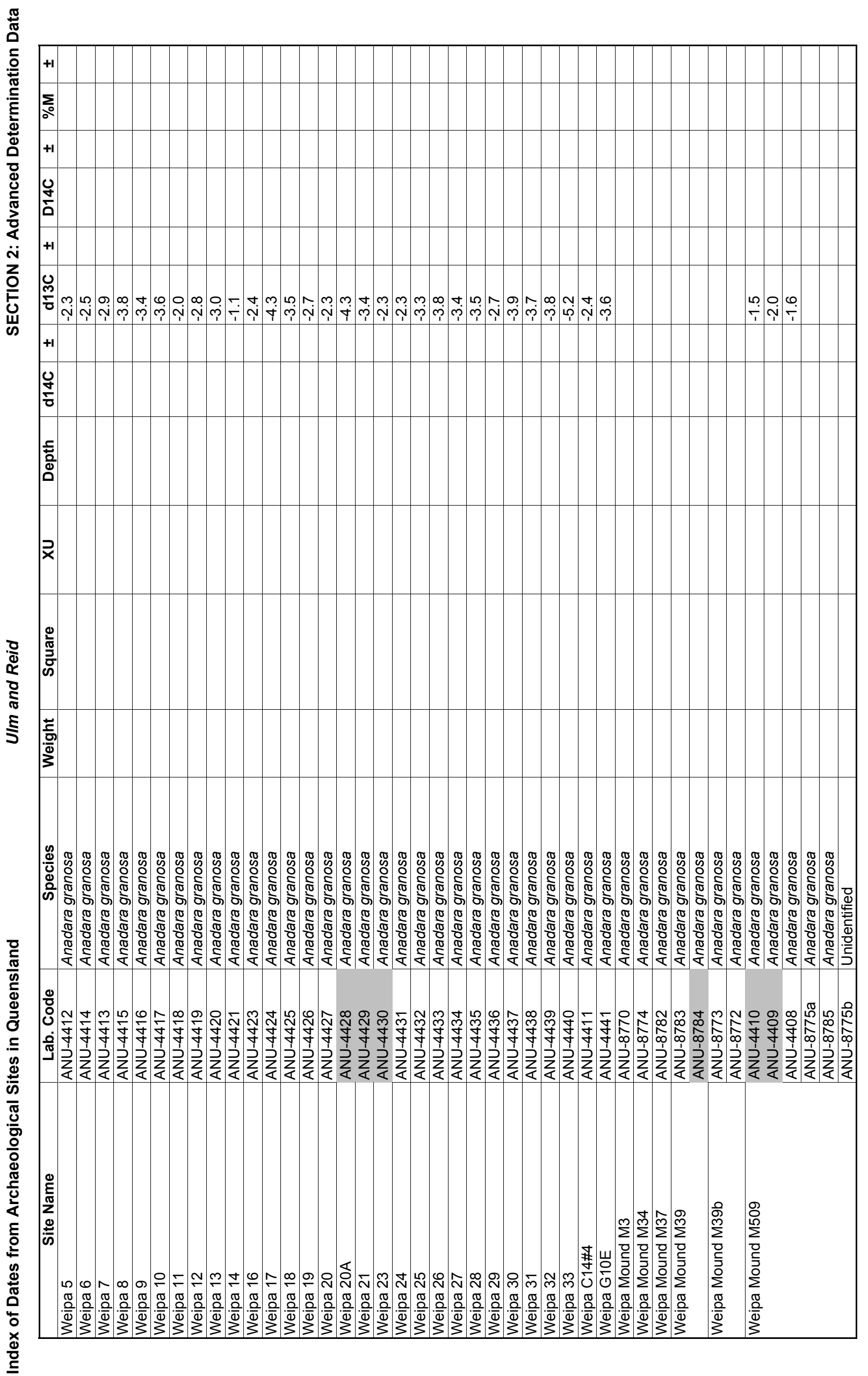




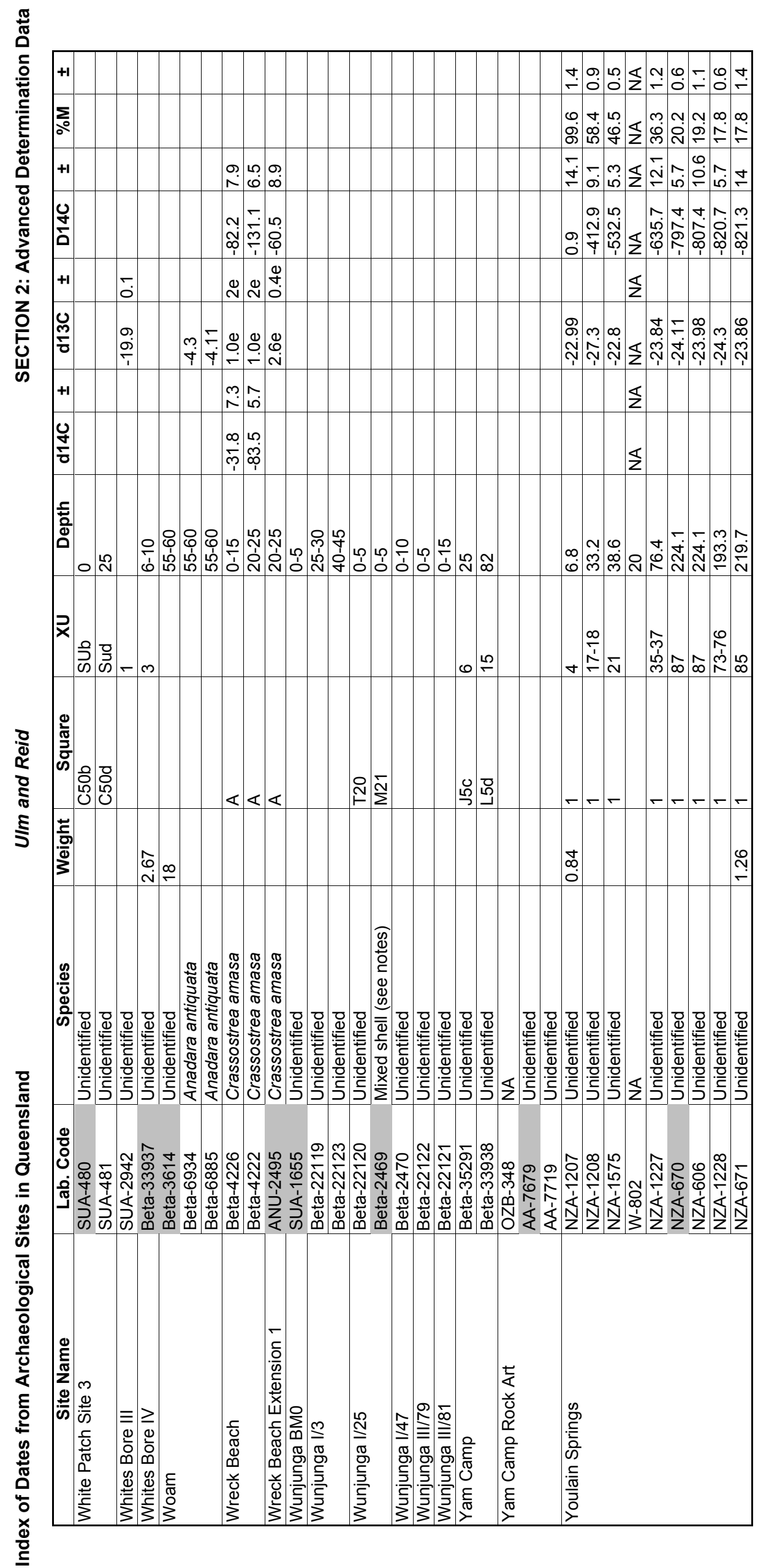




\section{SECTION 3:}

Notes on Individual Determinations 


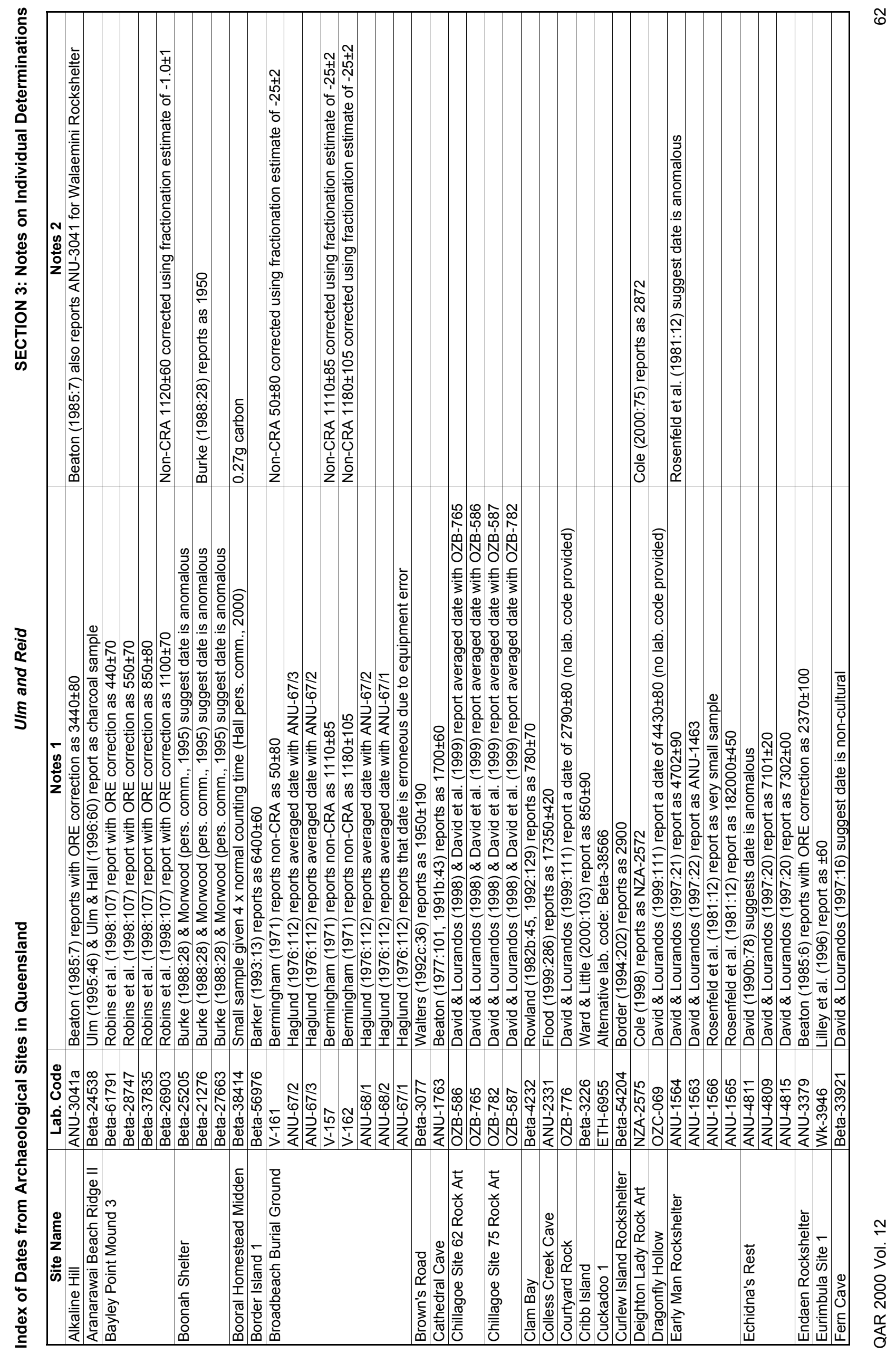



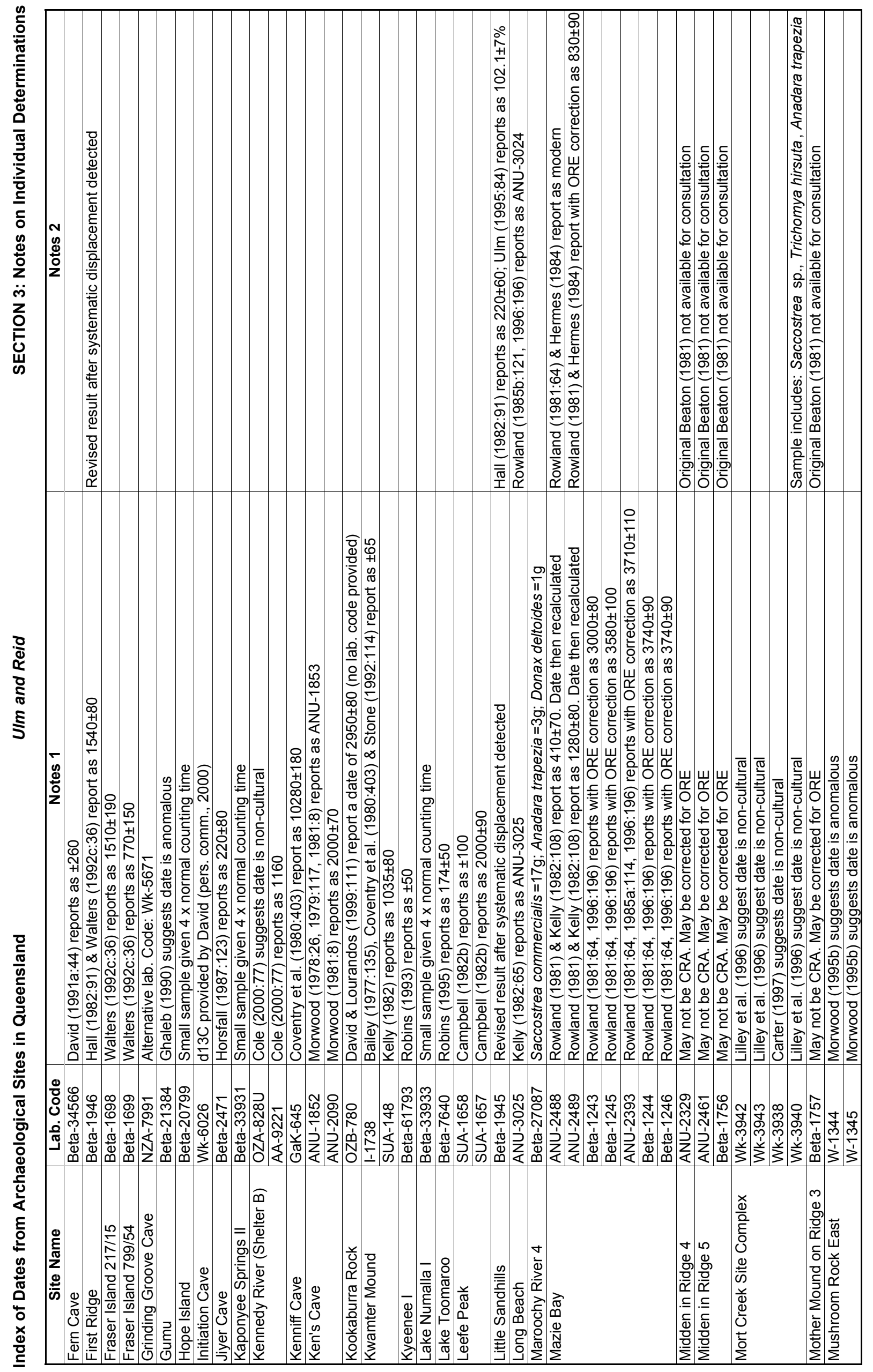


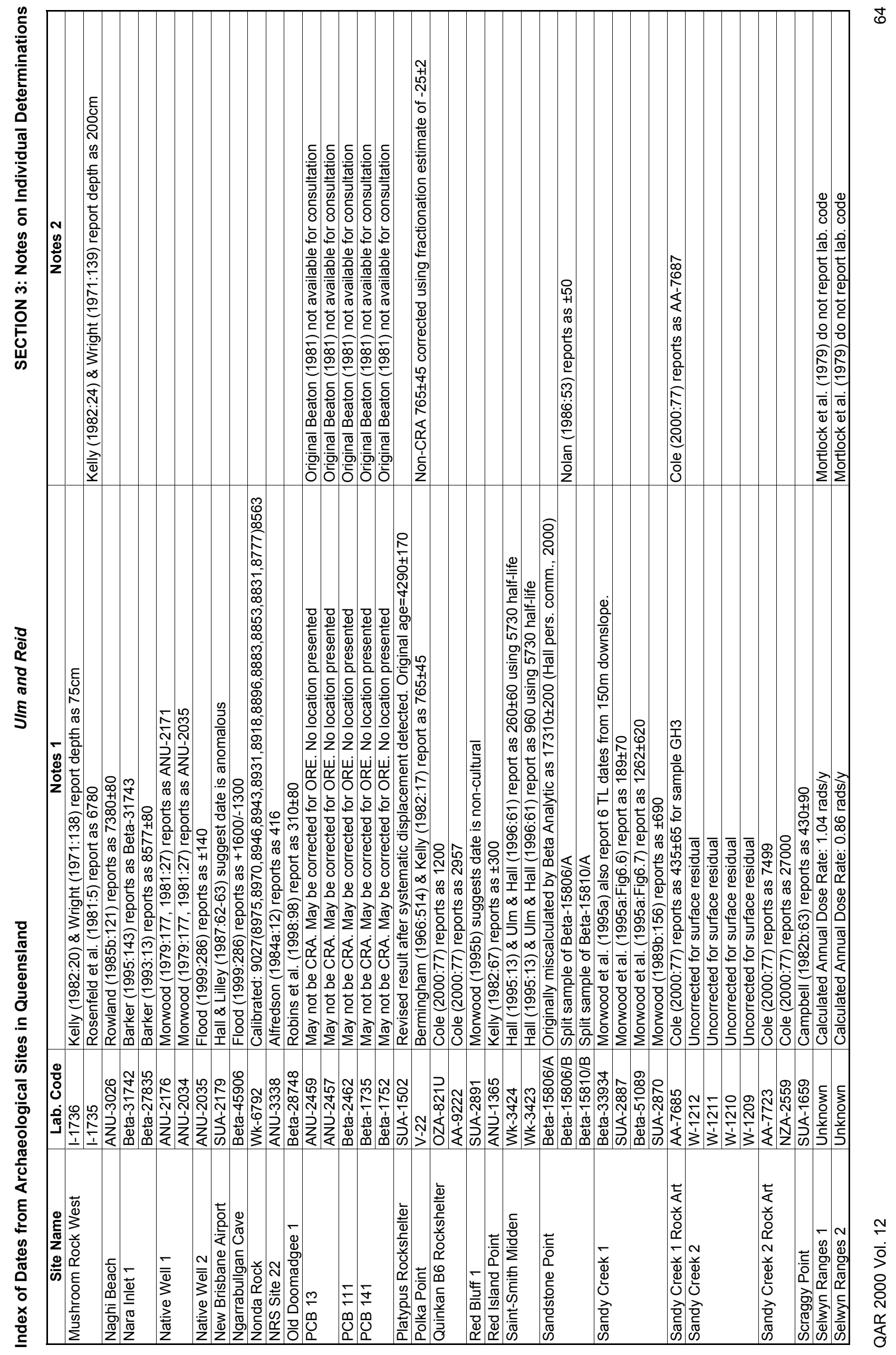




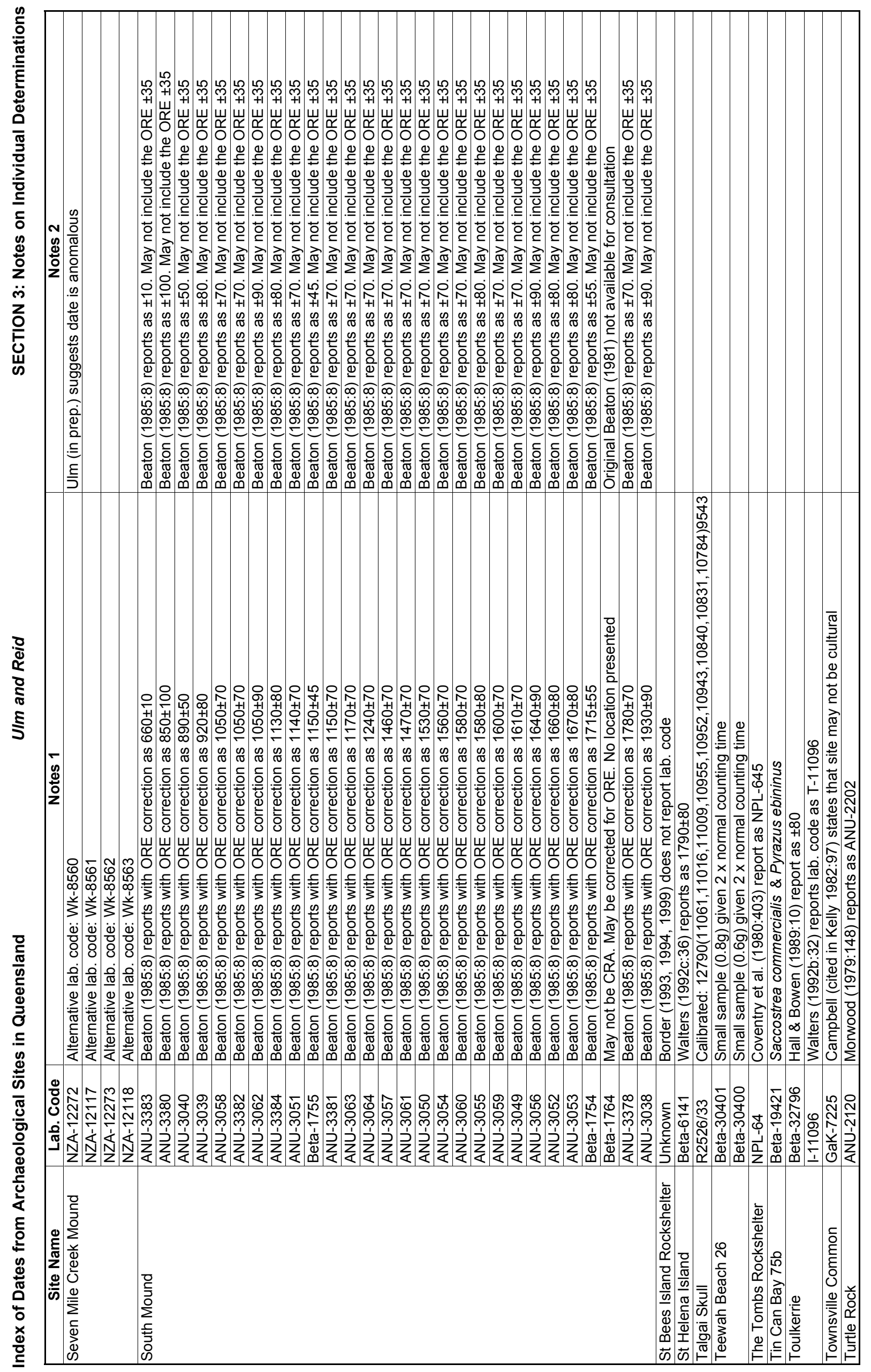

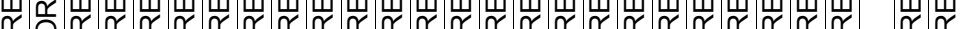
O O O ×

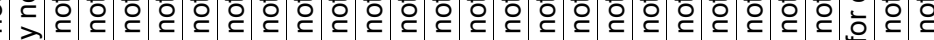

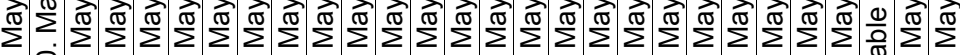
o

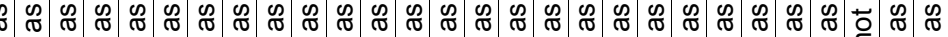

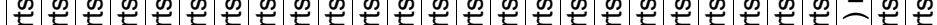

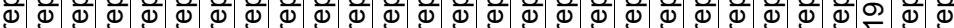

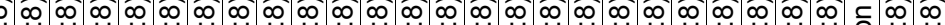

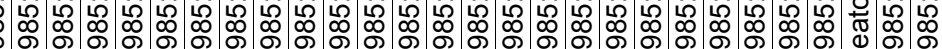

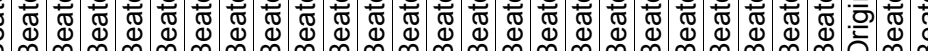

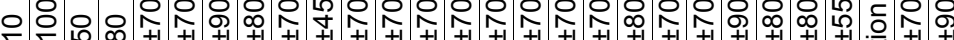
作

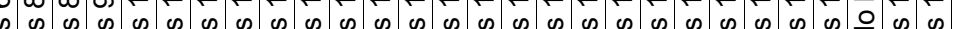
ᄃ ᄃ ᄃ ᄃ ᄃ

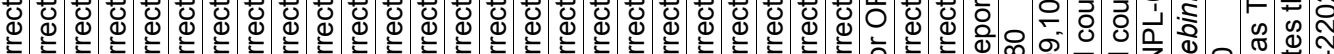

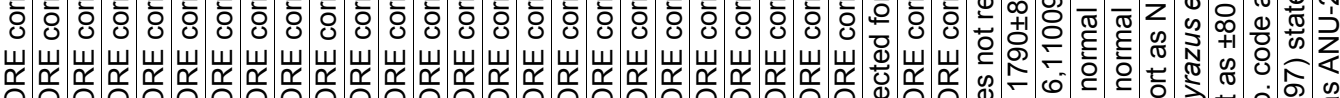

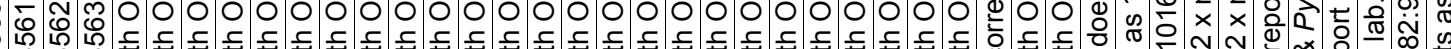

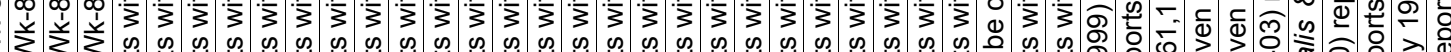

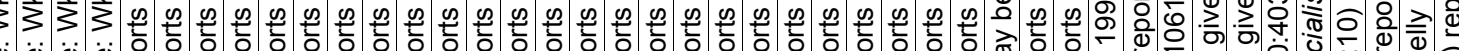
过

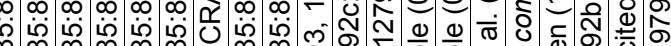

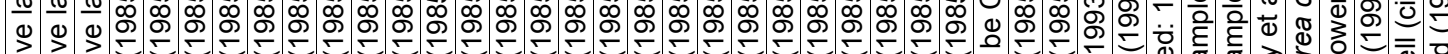

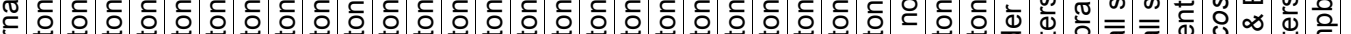

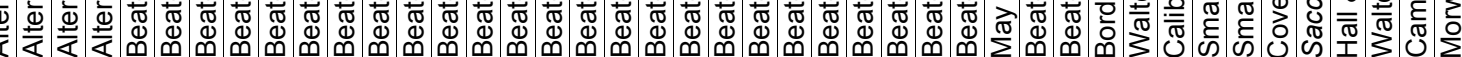

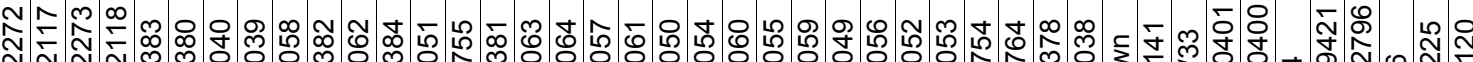
$\sim N N$ N

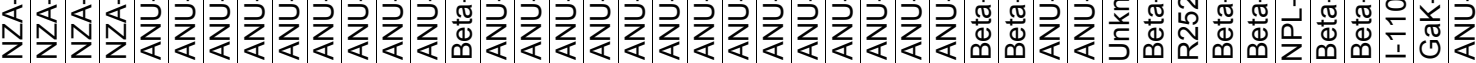




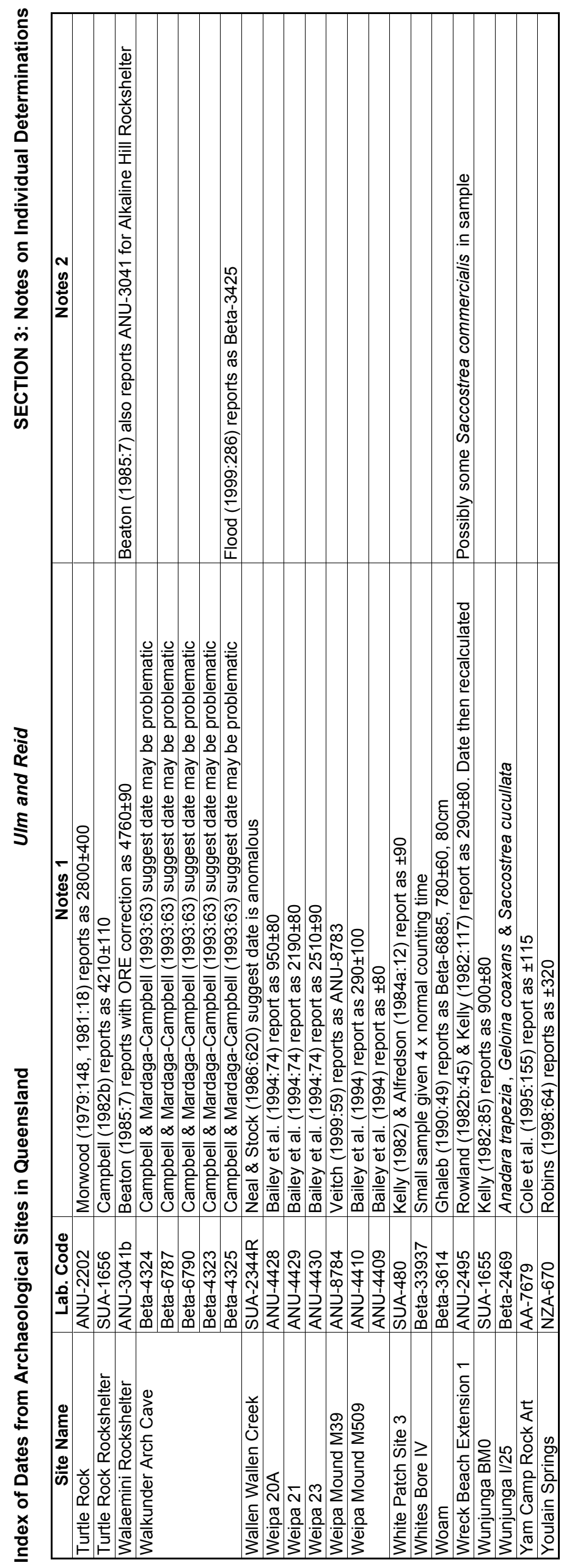




\section{SECTION 4:}

\section{Site Information}




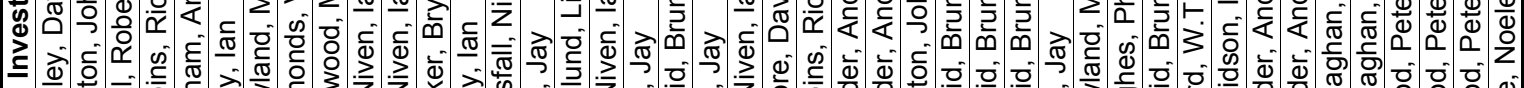

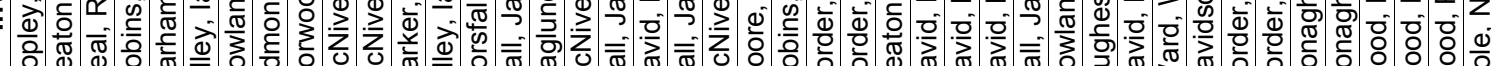

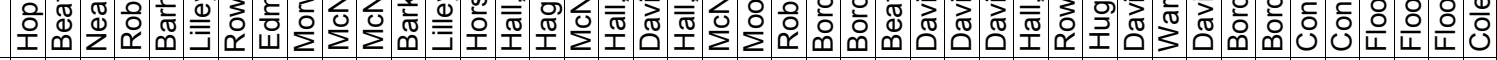

ธ

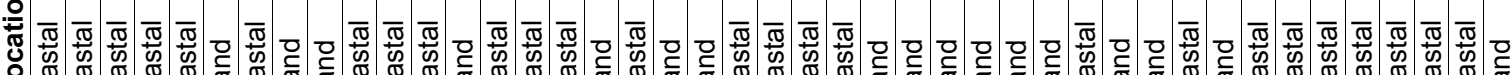

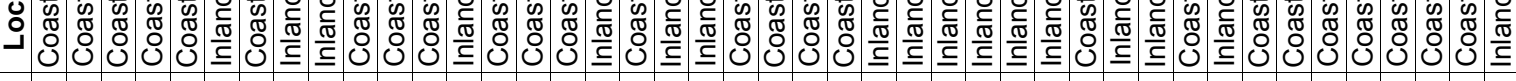

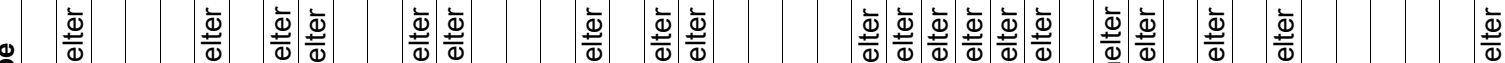

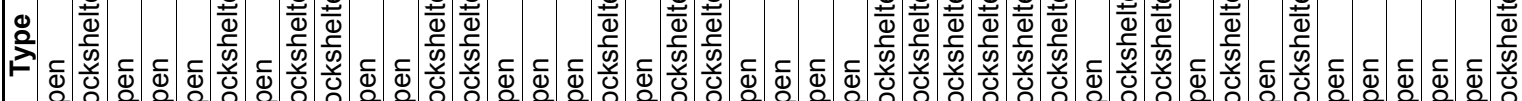

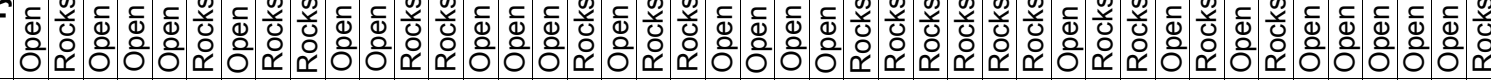
용

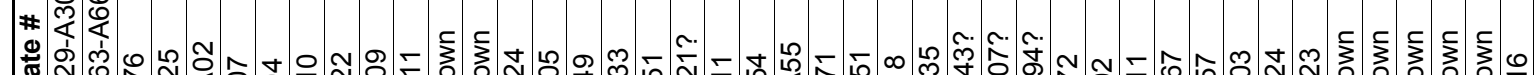

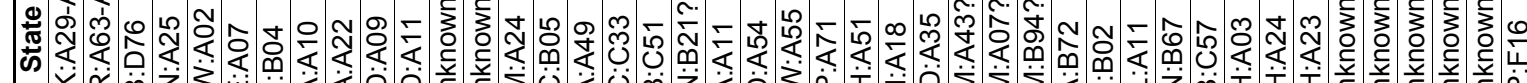

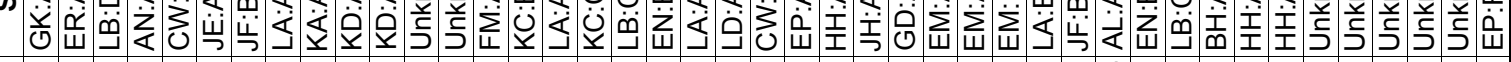
-

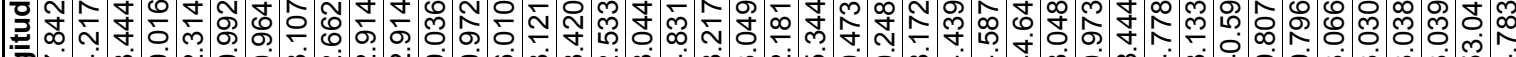

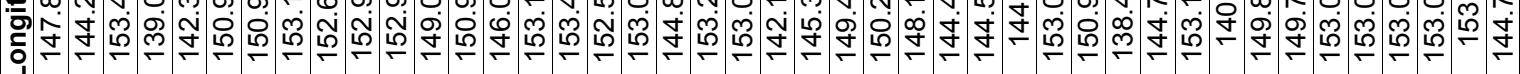

¿

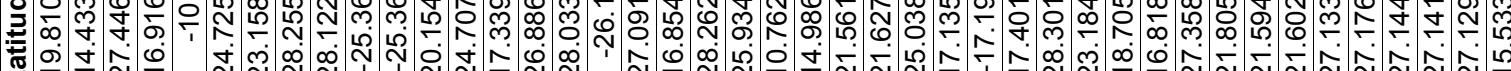
తึ

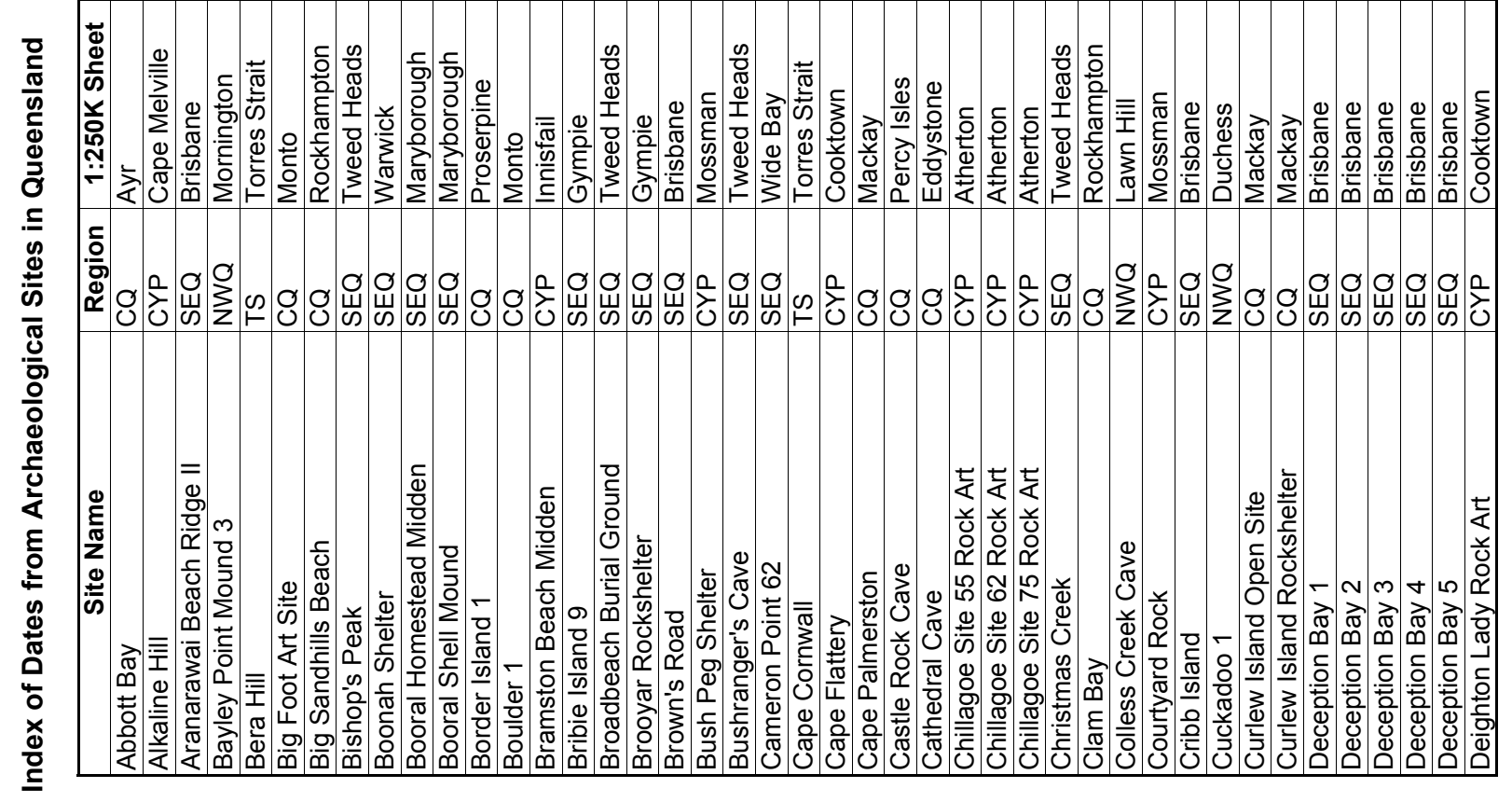

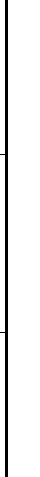




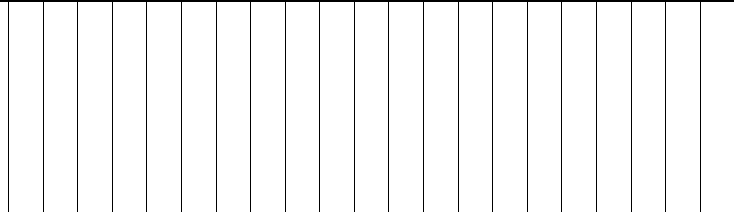

¿

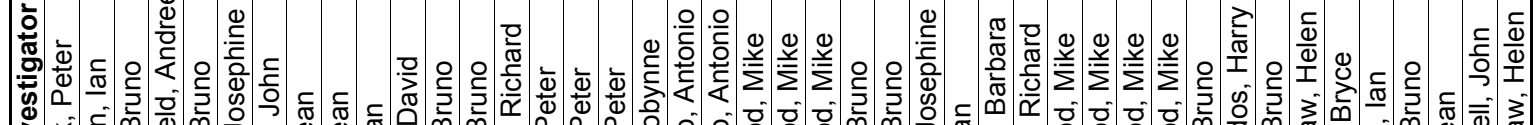

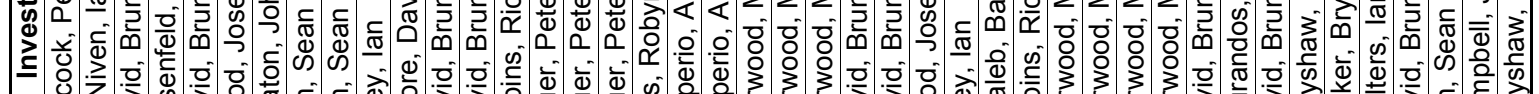

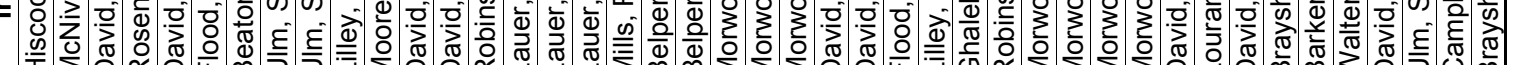

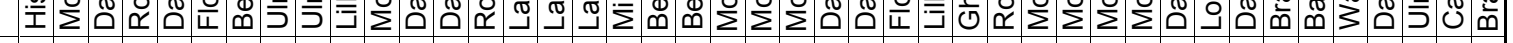

흔

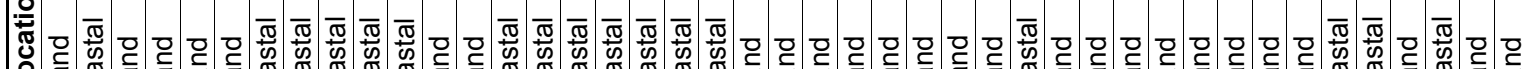
ดำ

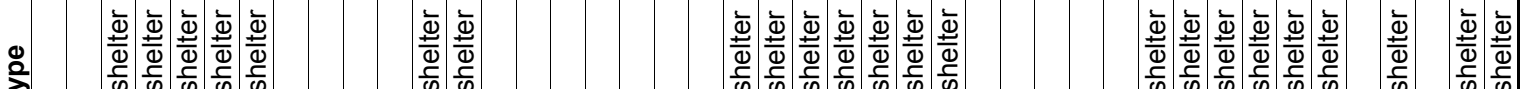

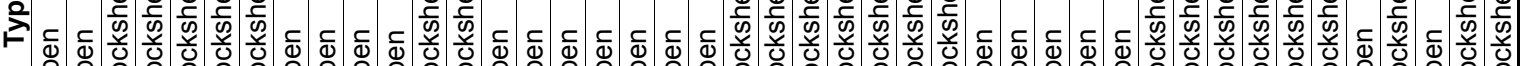

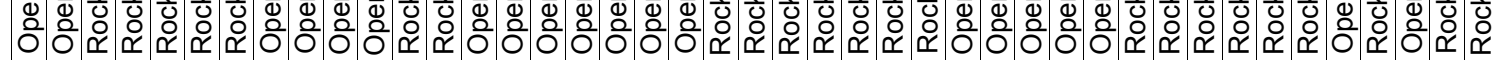

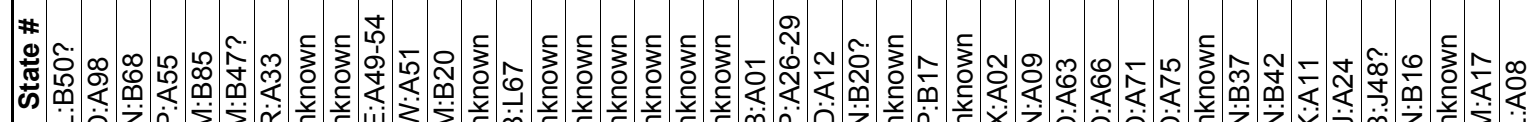

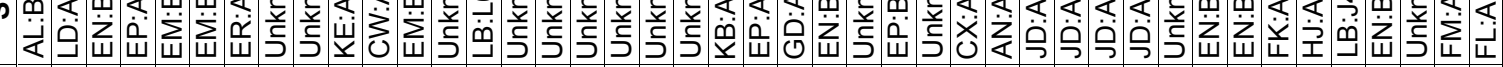

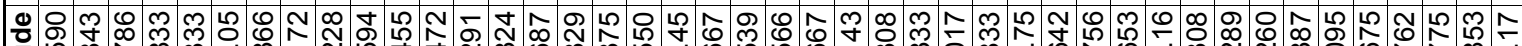

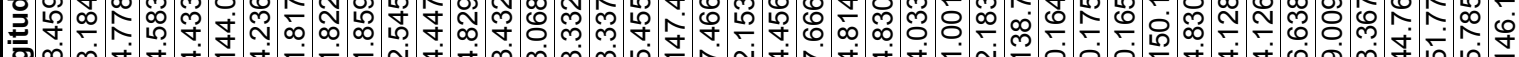

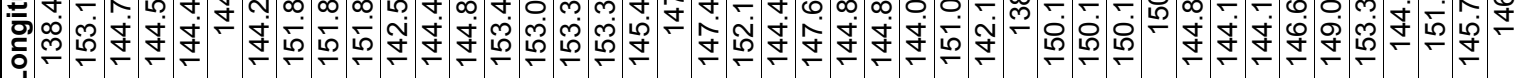

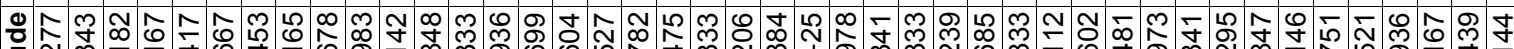

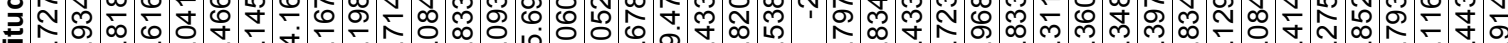

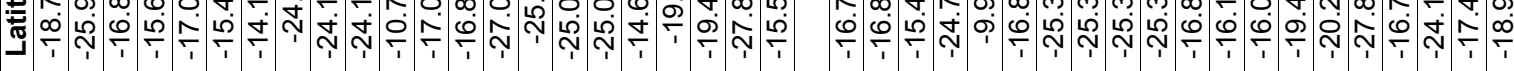

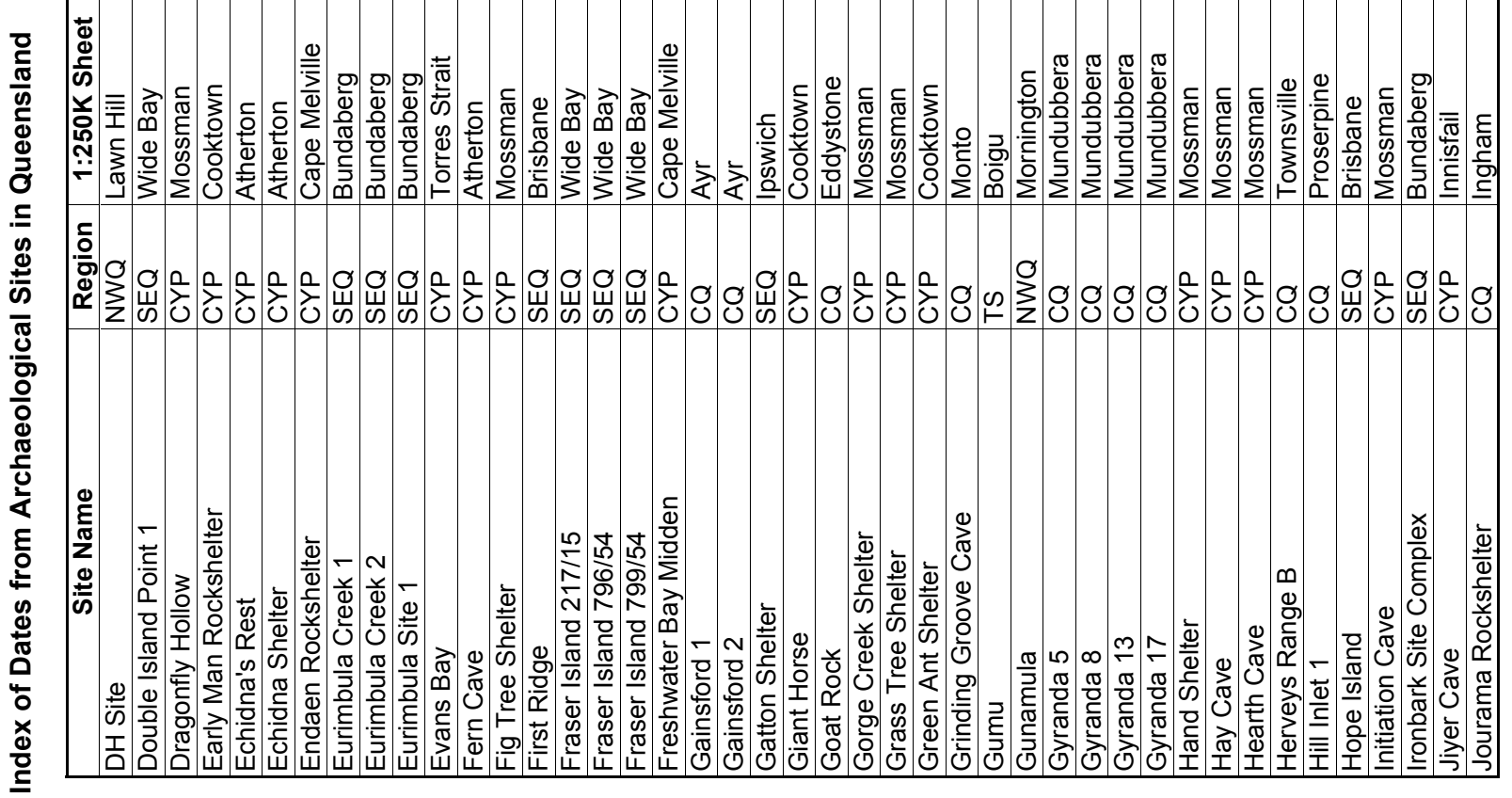


¿̀

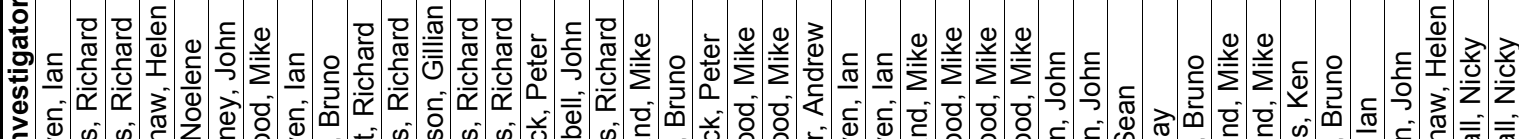

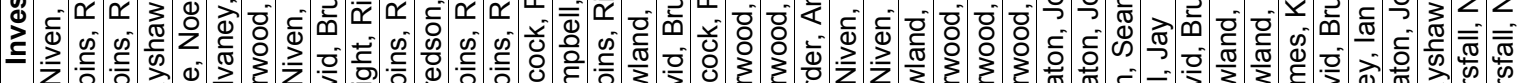

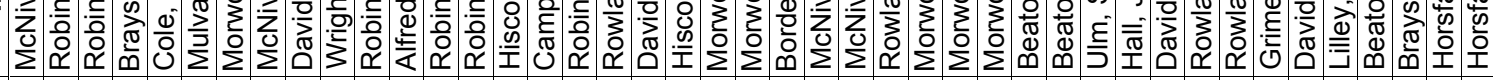

ธํ.

产

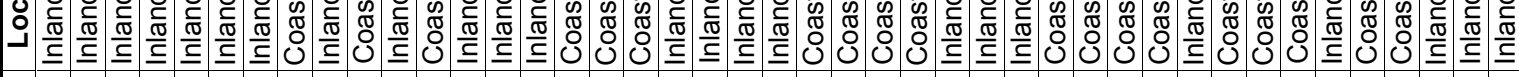

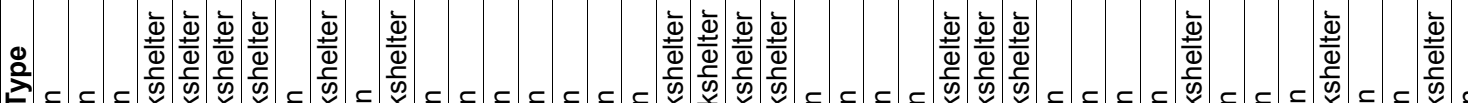

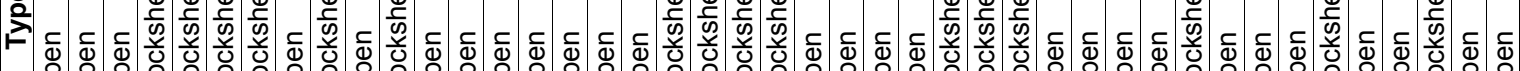

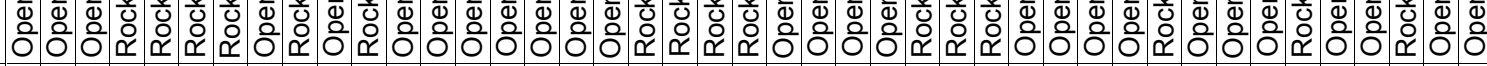

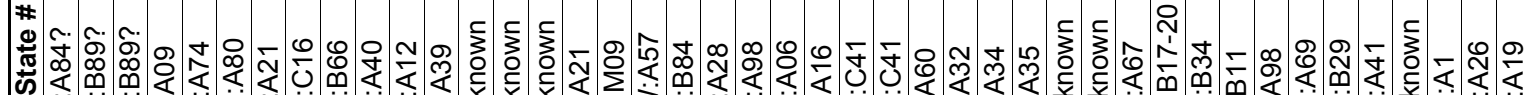

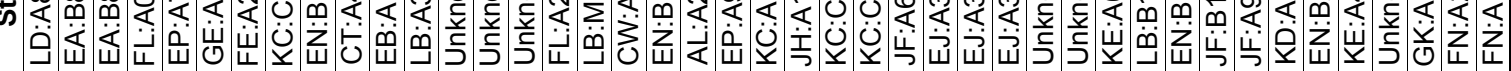

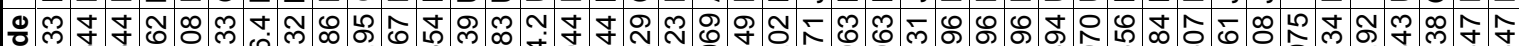

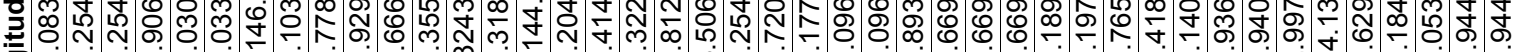

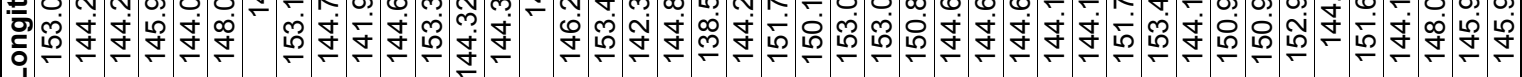

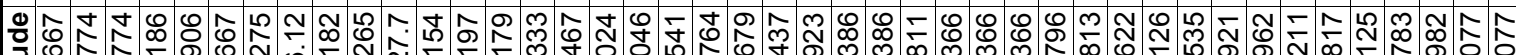

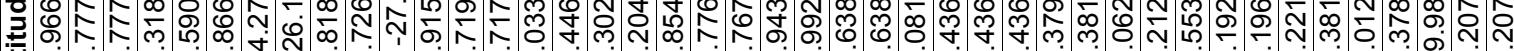

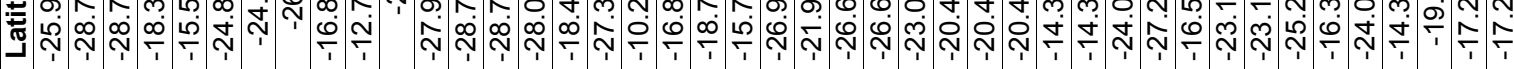

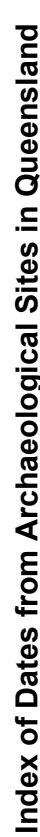

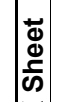

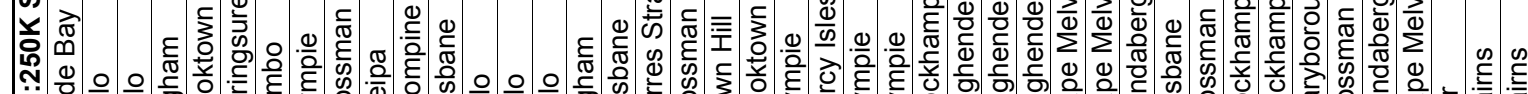

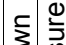

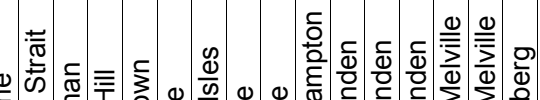

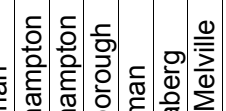

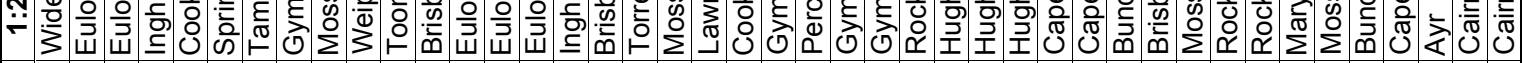

흐

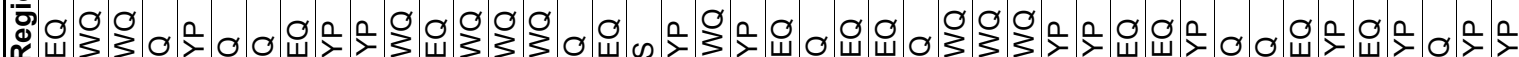

ॠ

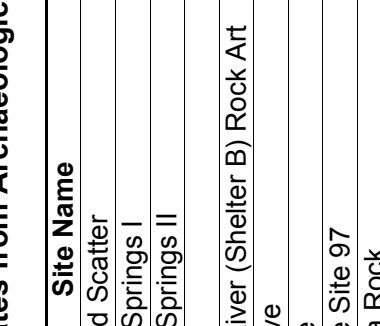

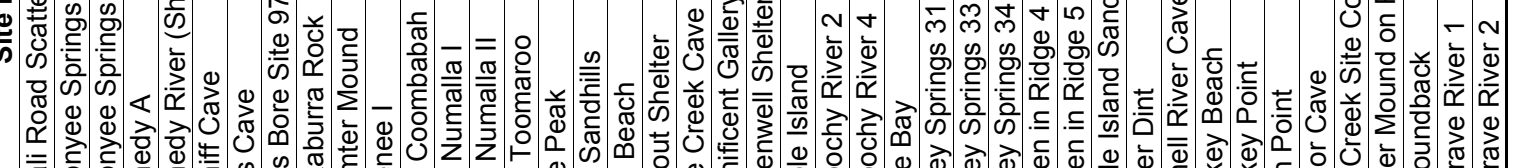

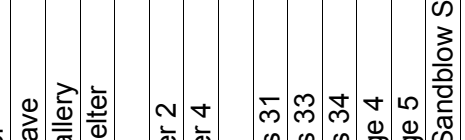

क्ष

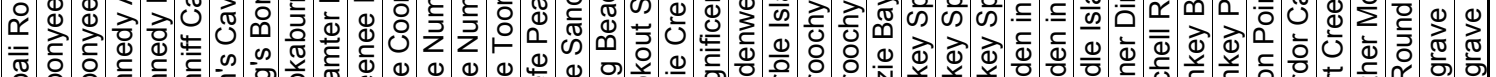

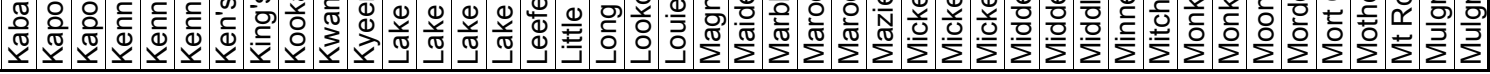


E

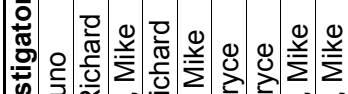

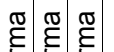

Zे

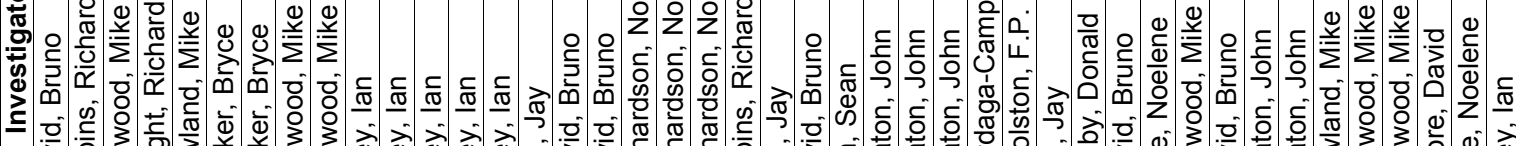

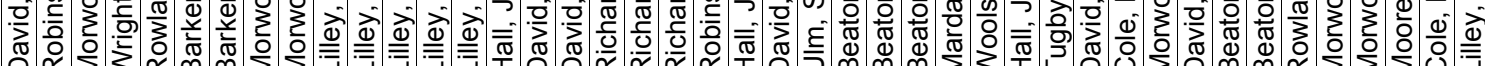

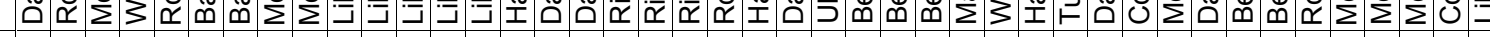

ธิ

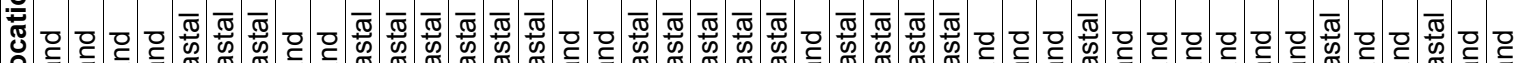

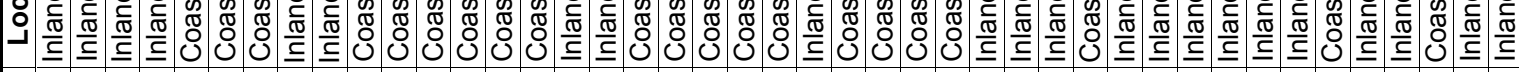

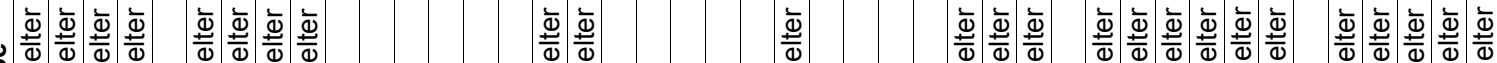

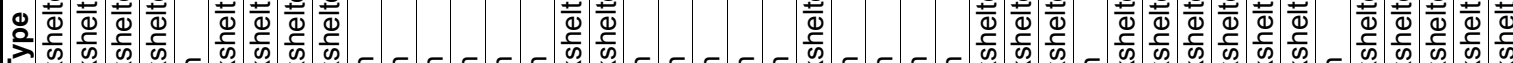

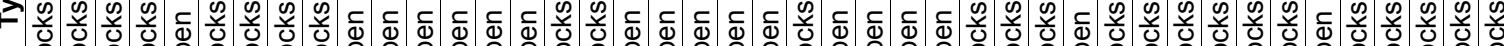

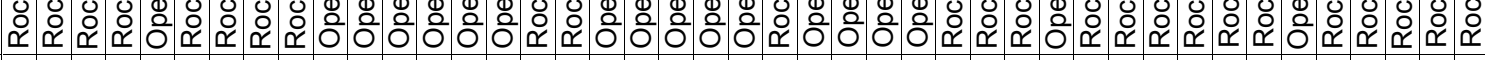

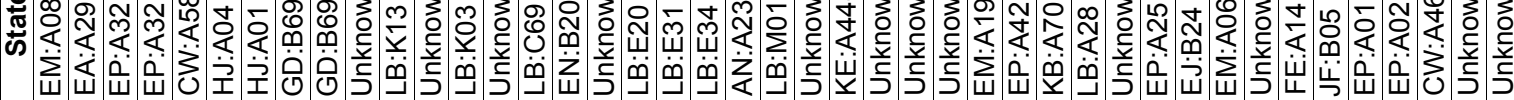
\&

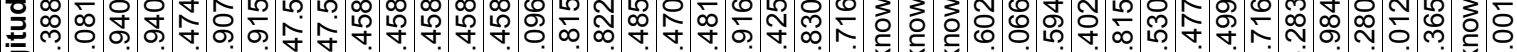

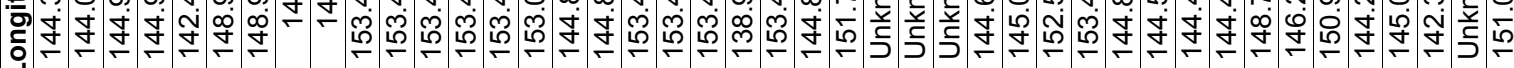

-

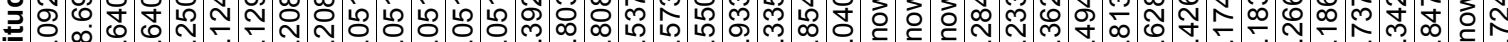

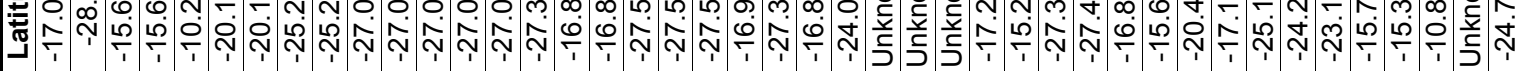

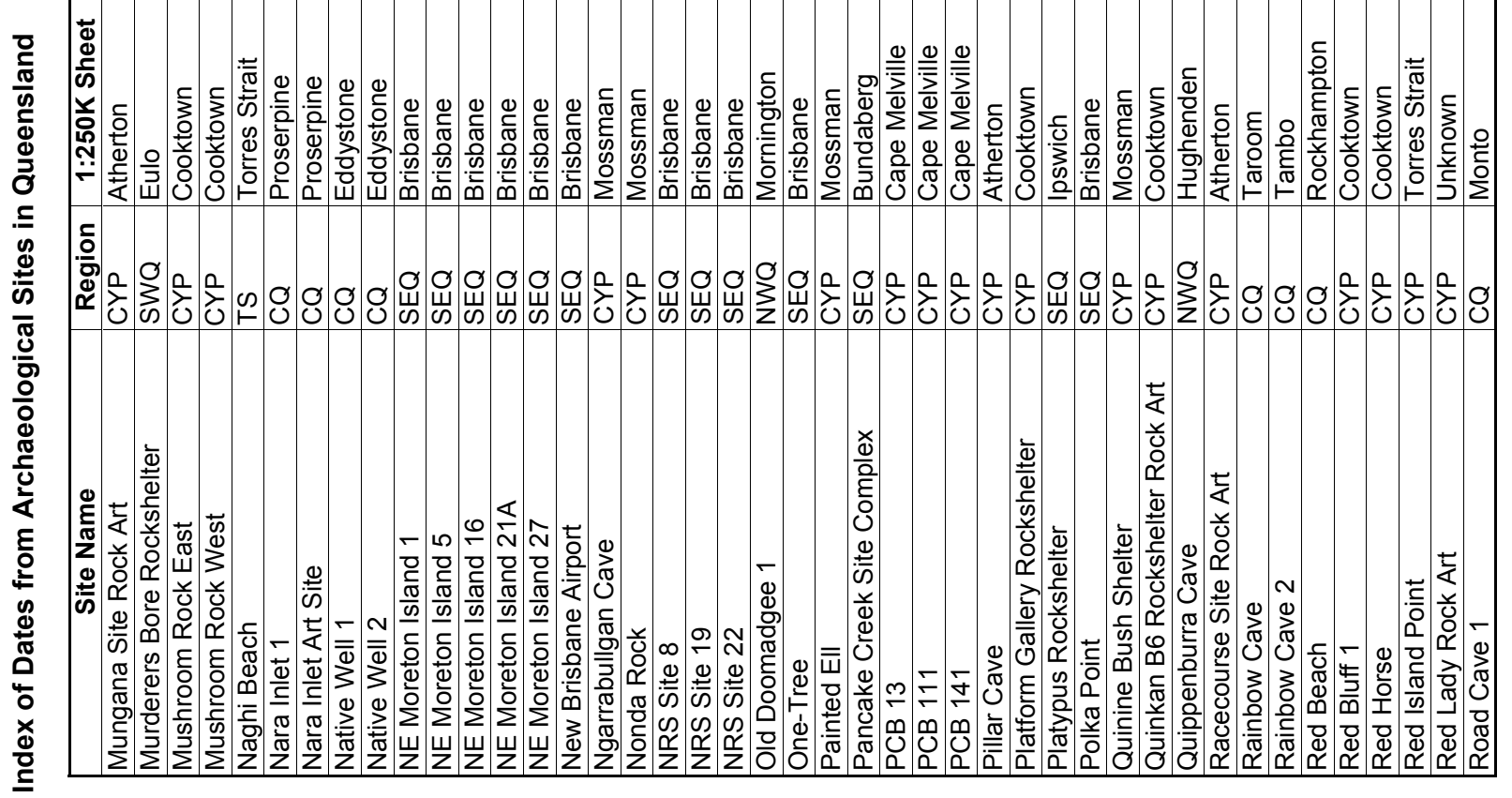


-

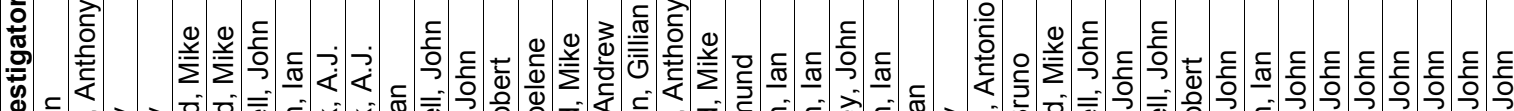

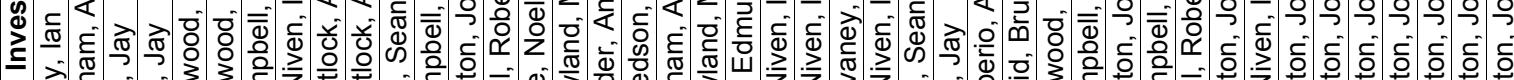

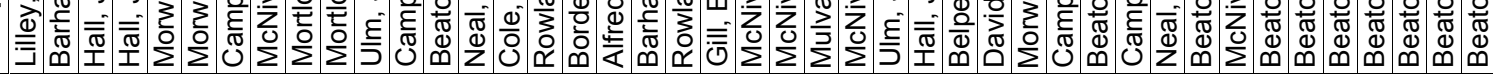

ธ.

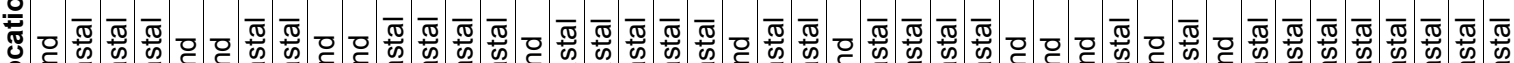

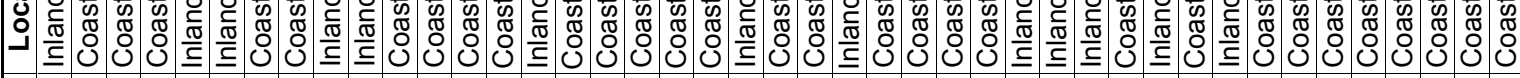

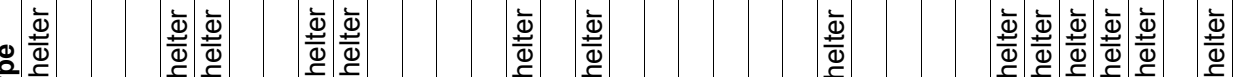

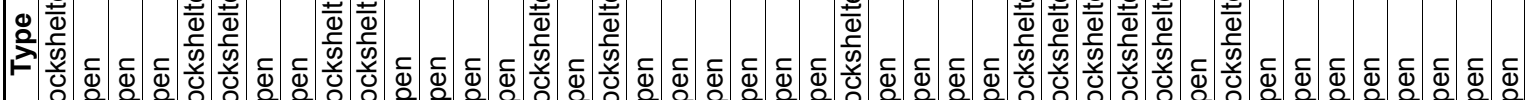

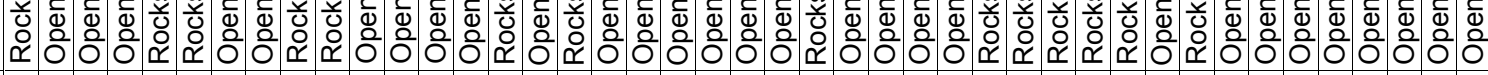

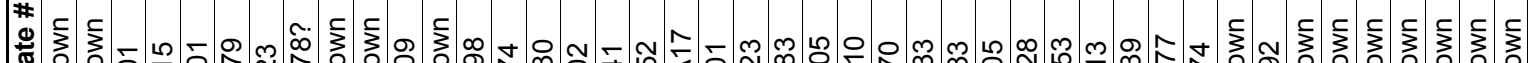

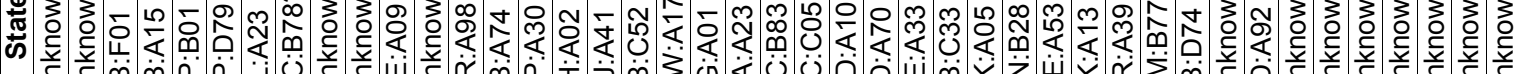

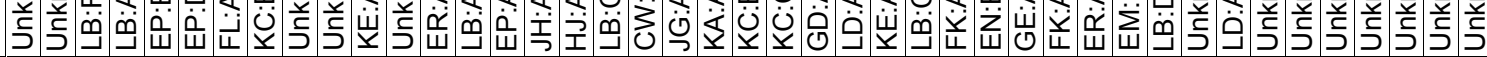

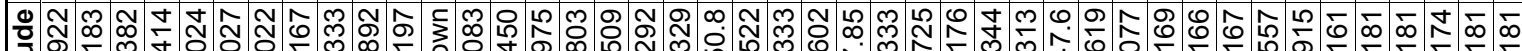

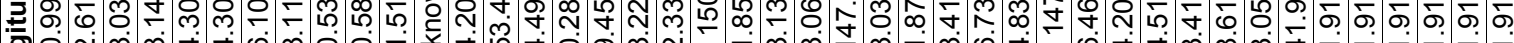

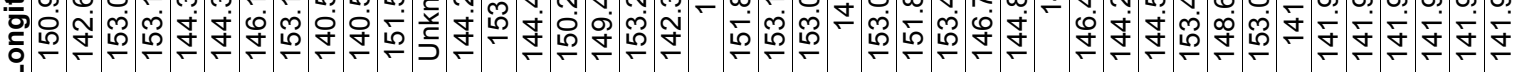

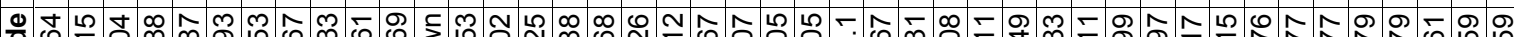

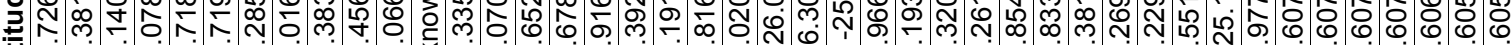

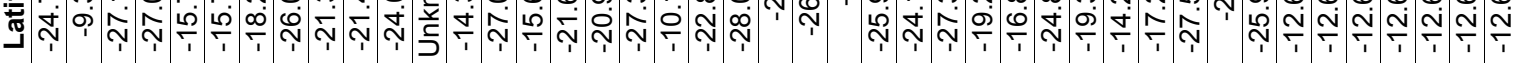

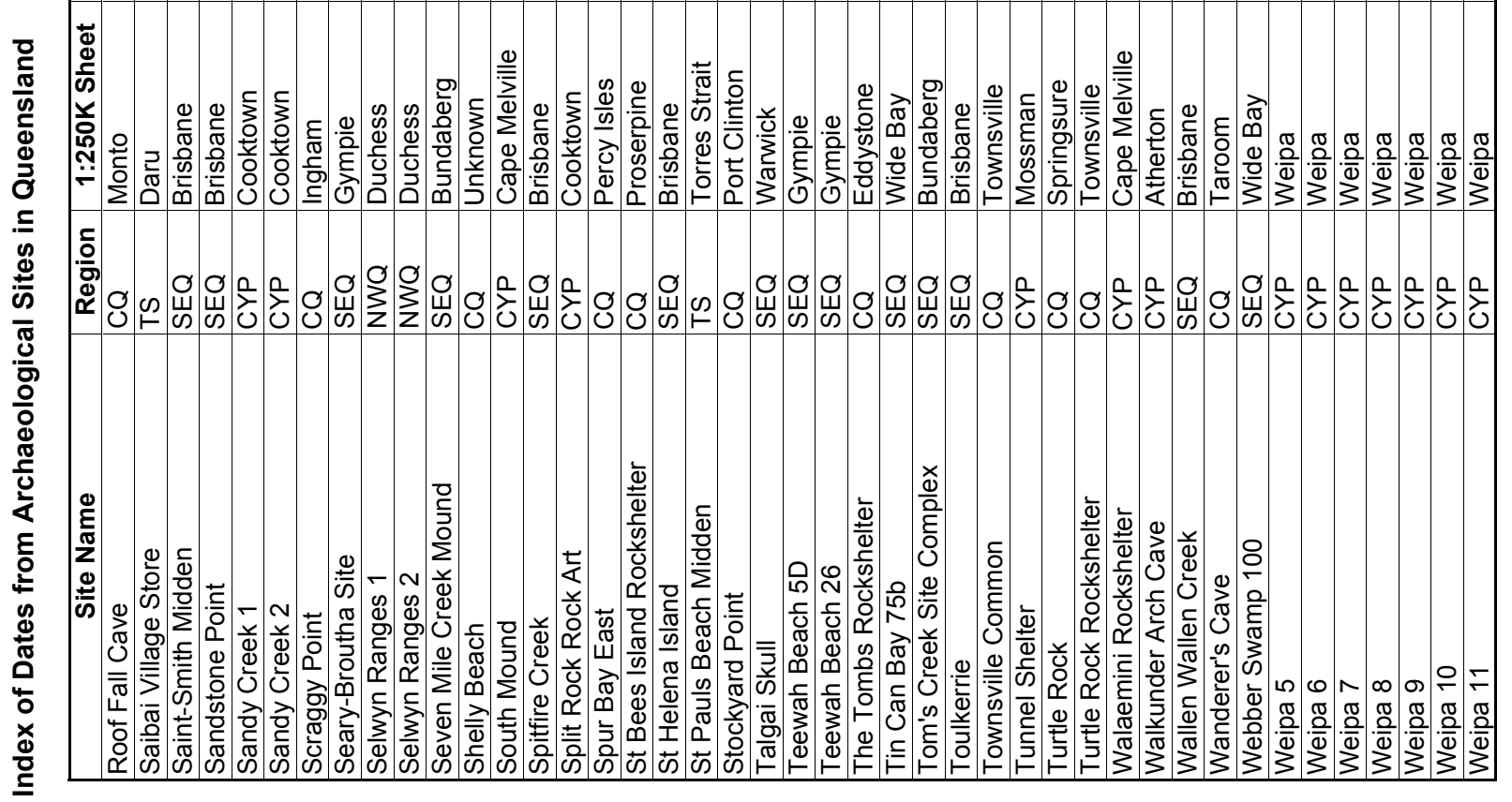




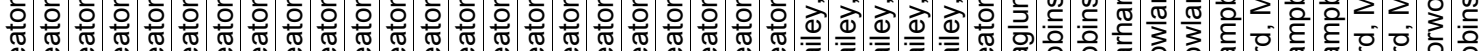
œ

ธ

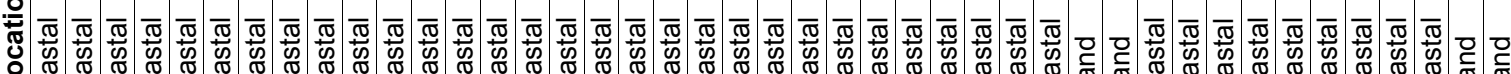

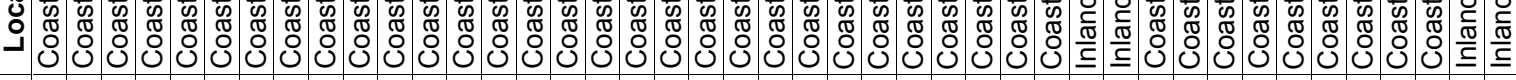

$\gtreqless$

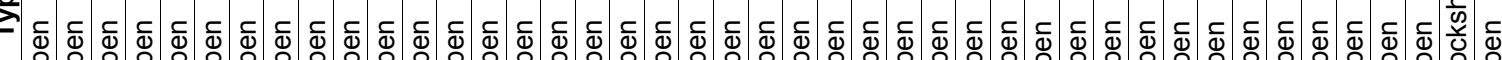

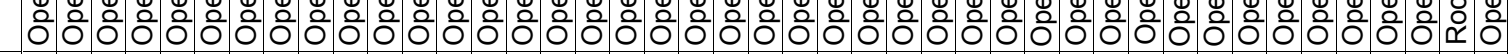

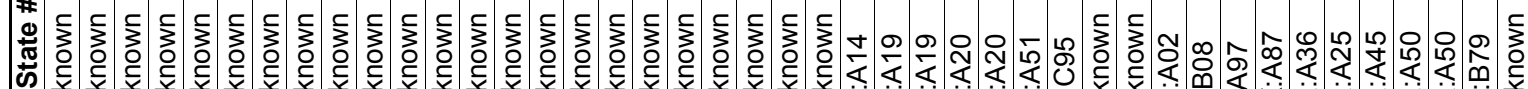

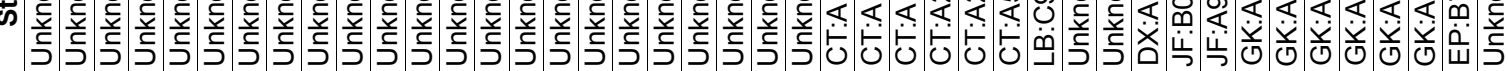

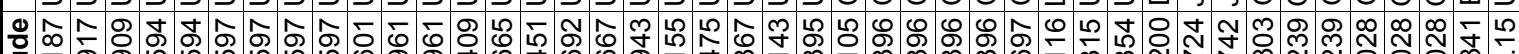

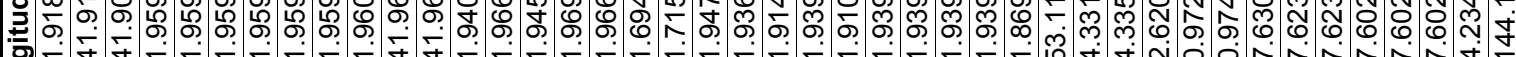

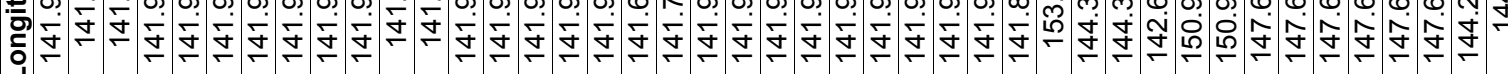

¿ñ

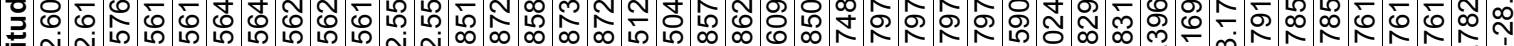
J゙

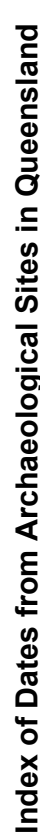

$\stackrel{\square}{\Phi}$

蒙

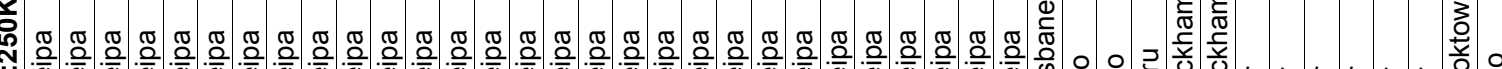

:

б

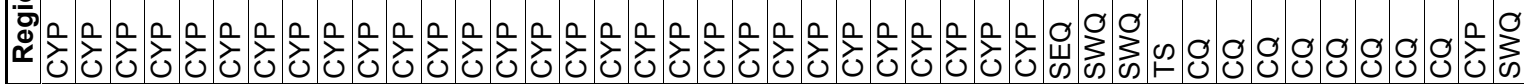

\&

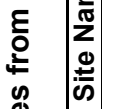

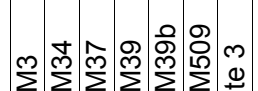

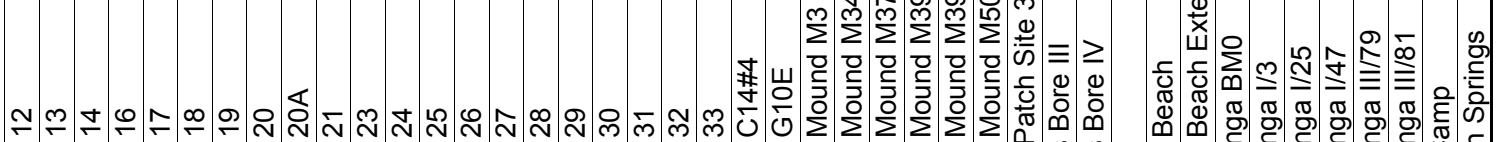

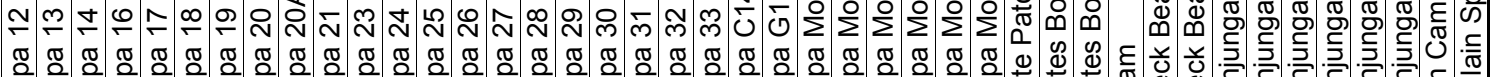

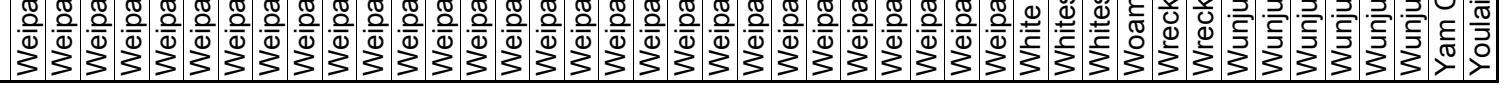



ㅎํ 응 $\frac{⿱ 亠}{\frac{\alpha}{\delta}}$ 


\section{SECTION 5:}

\section{Site References}



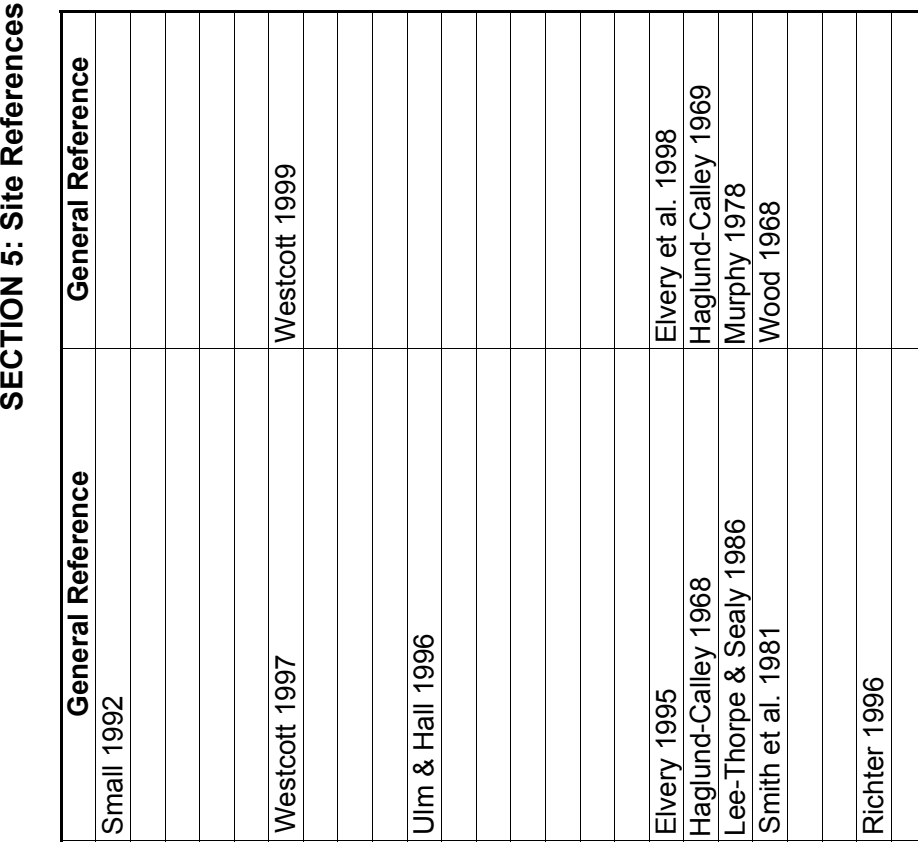

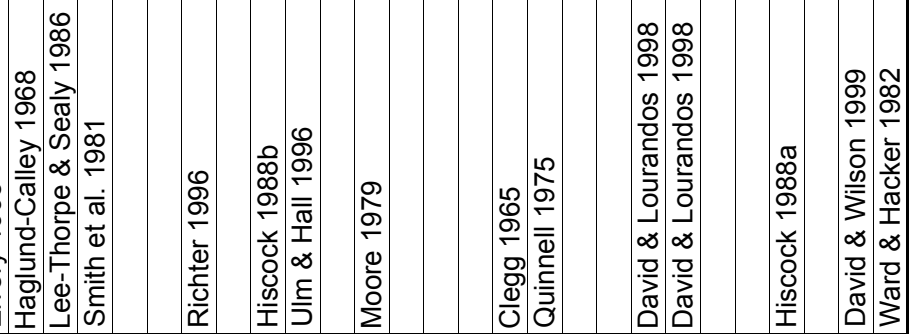
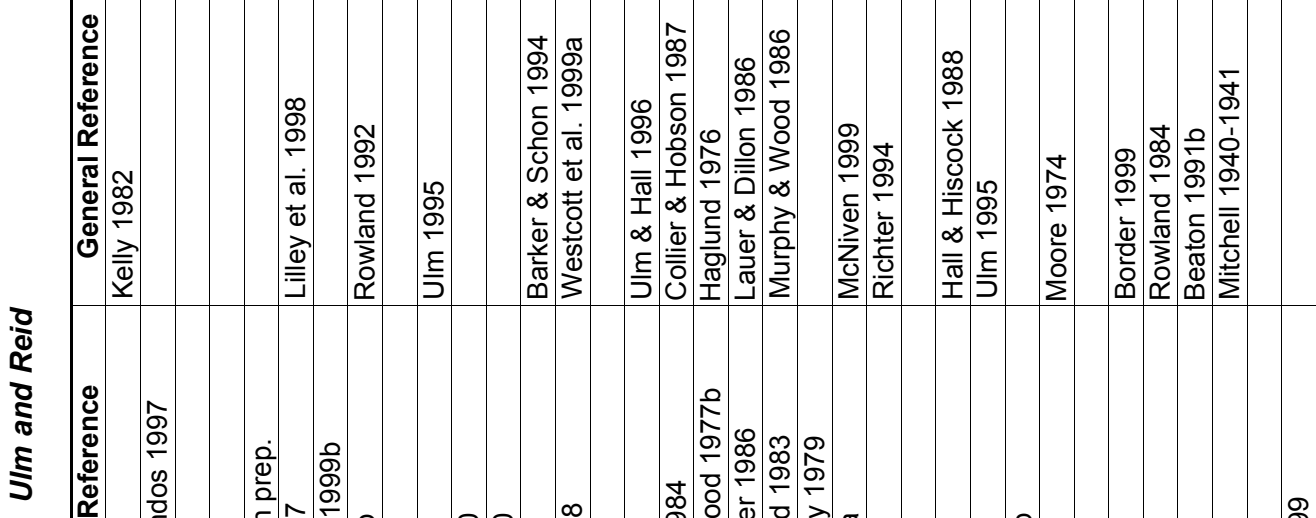

প.

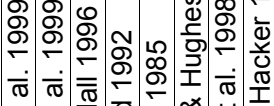

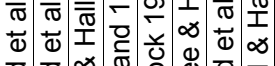

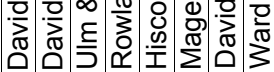

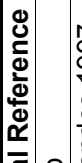

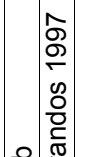

产

수

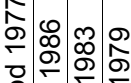

尽

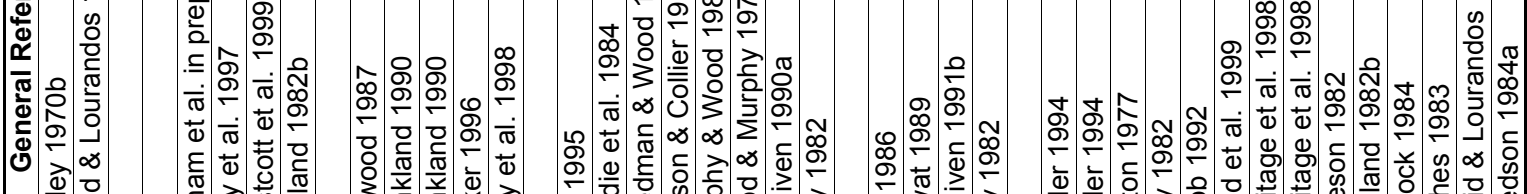

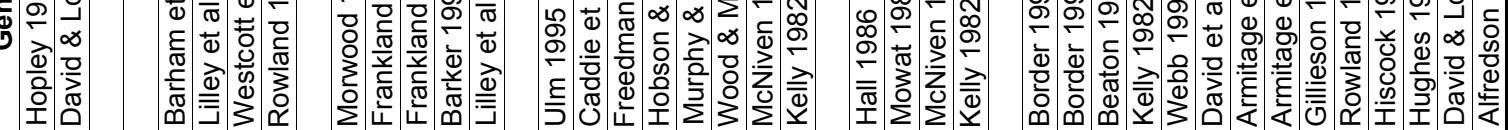

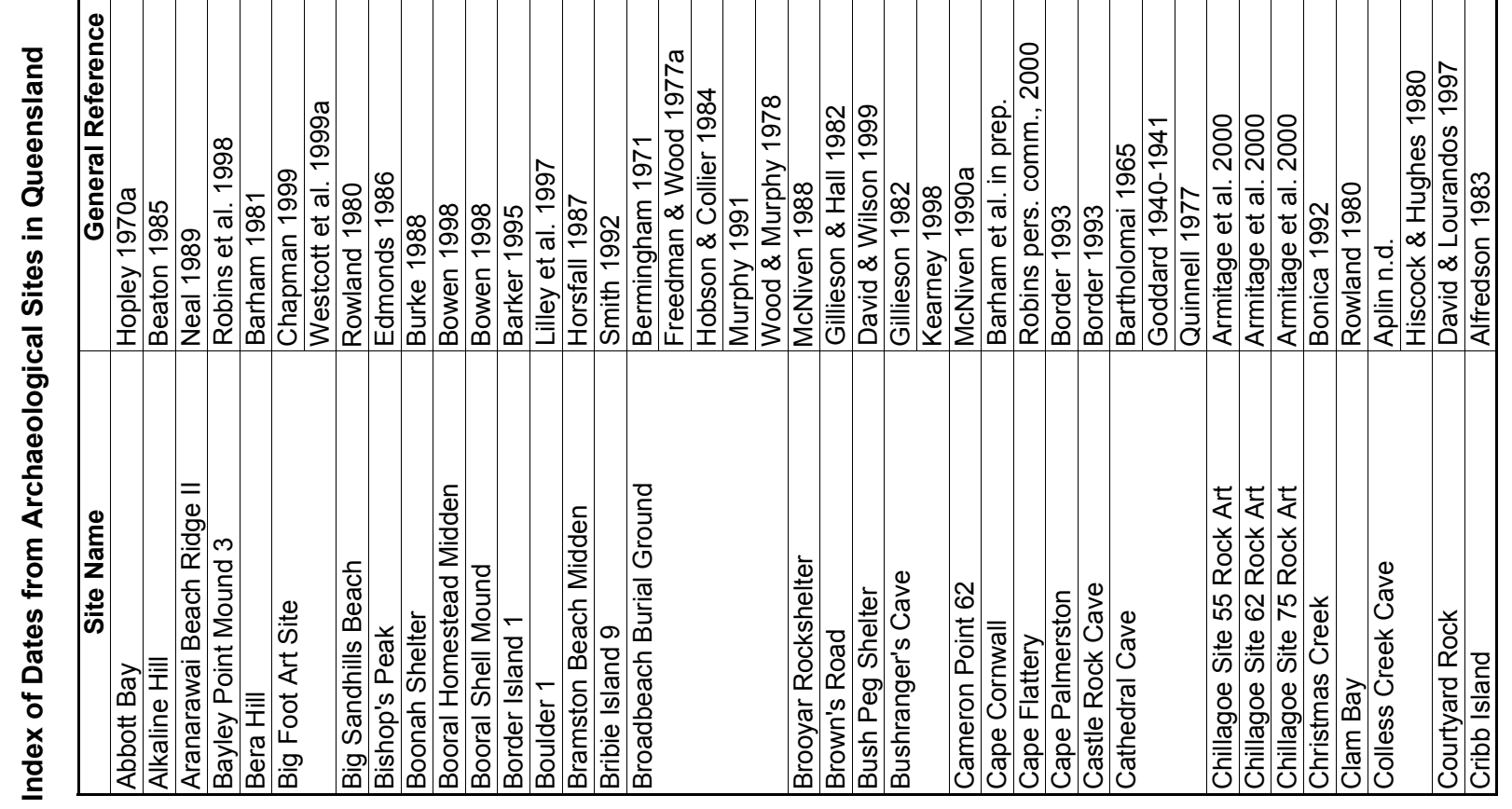

氛 

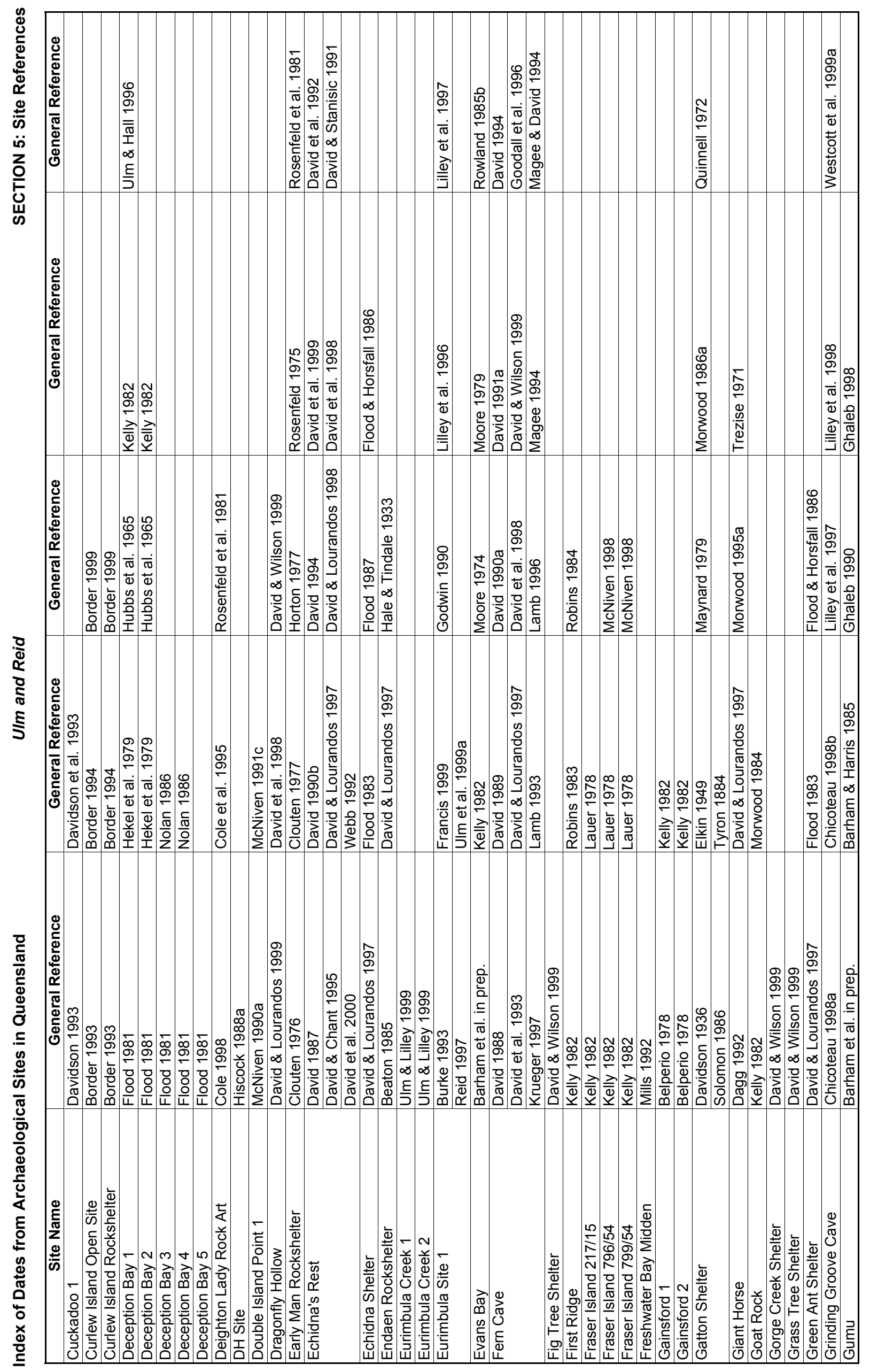


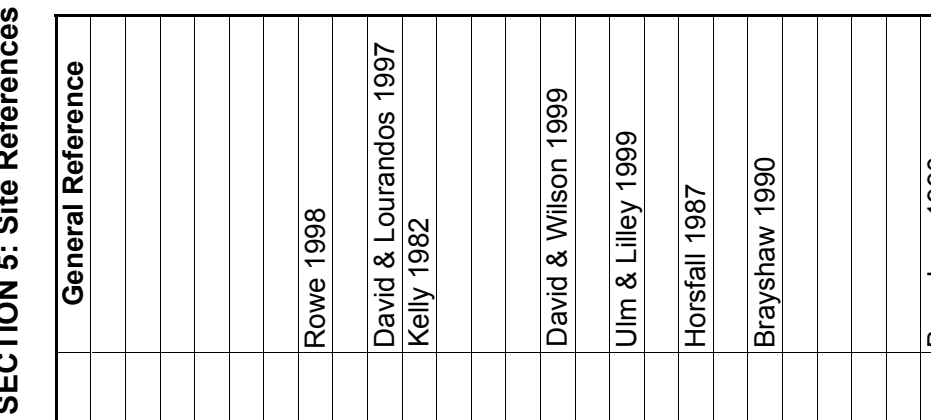

$\stackrel{8}{8}$

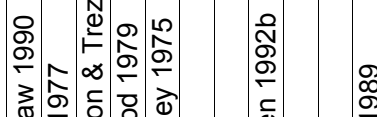

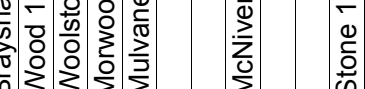

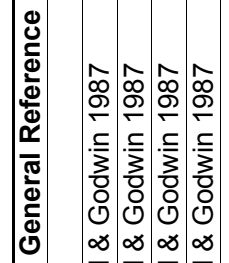

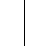

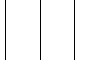

$3=$

.

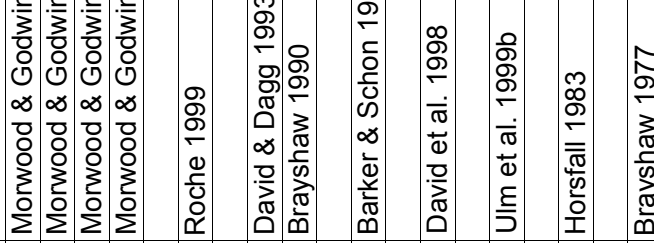

言

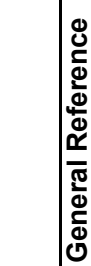

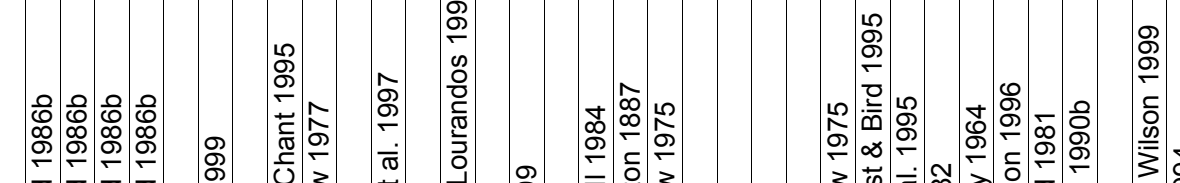

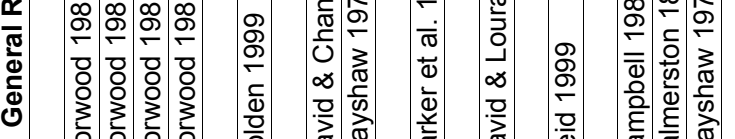

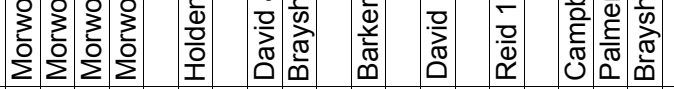

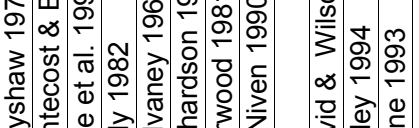

路㴵

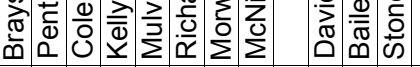

高

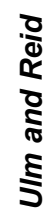

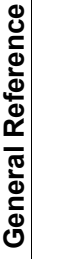

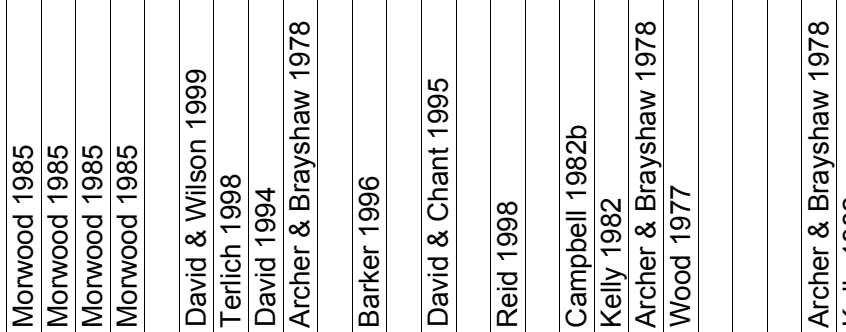

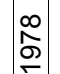

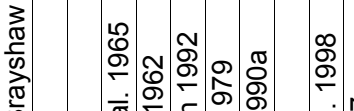

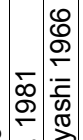

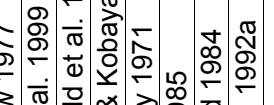

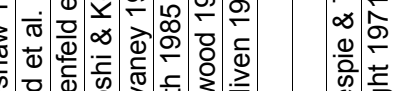

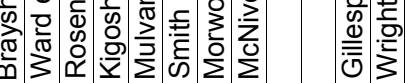

$$
\text { ( }
$$

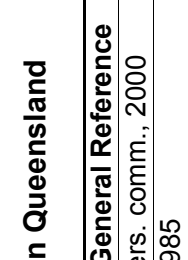

๕.

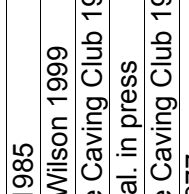

ำ

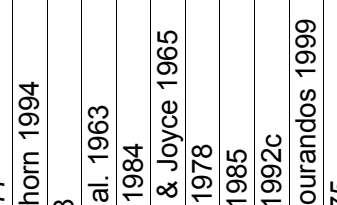

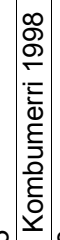

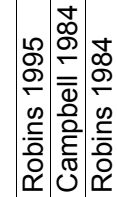

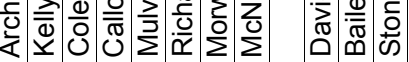

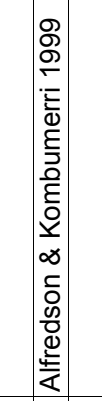




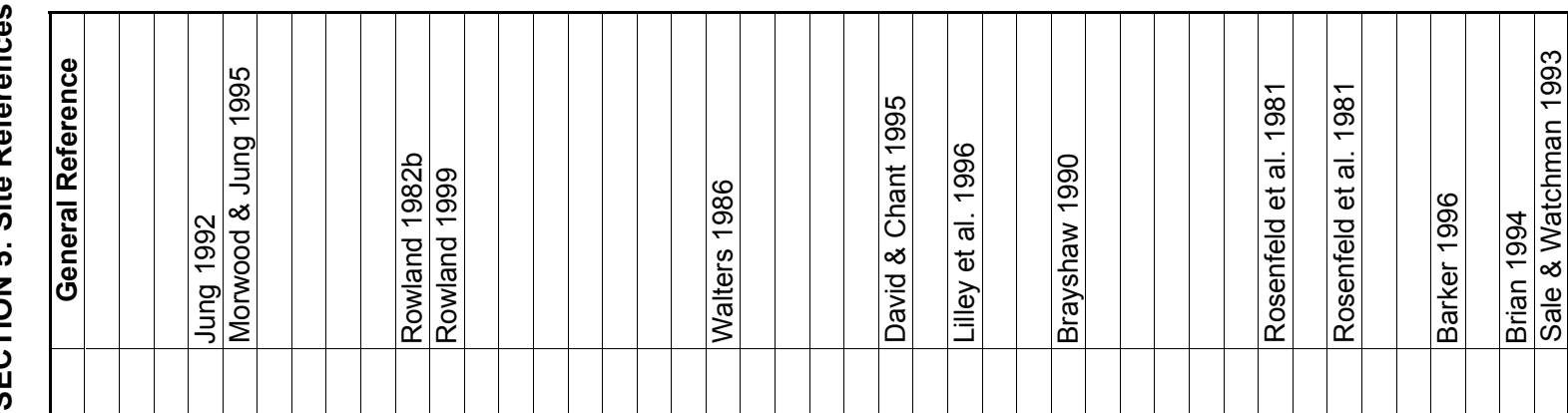

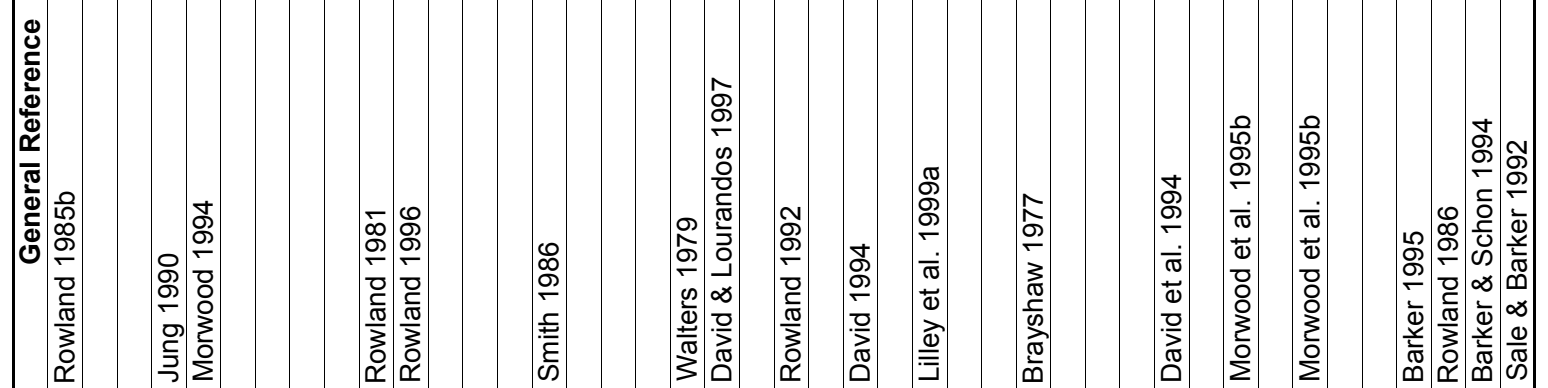
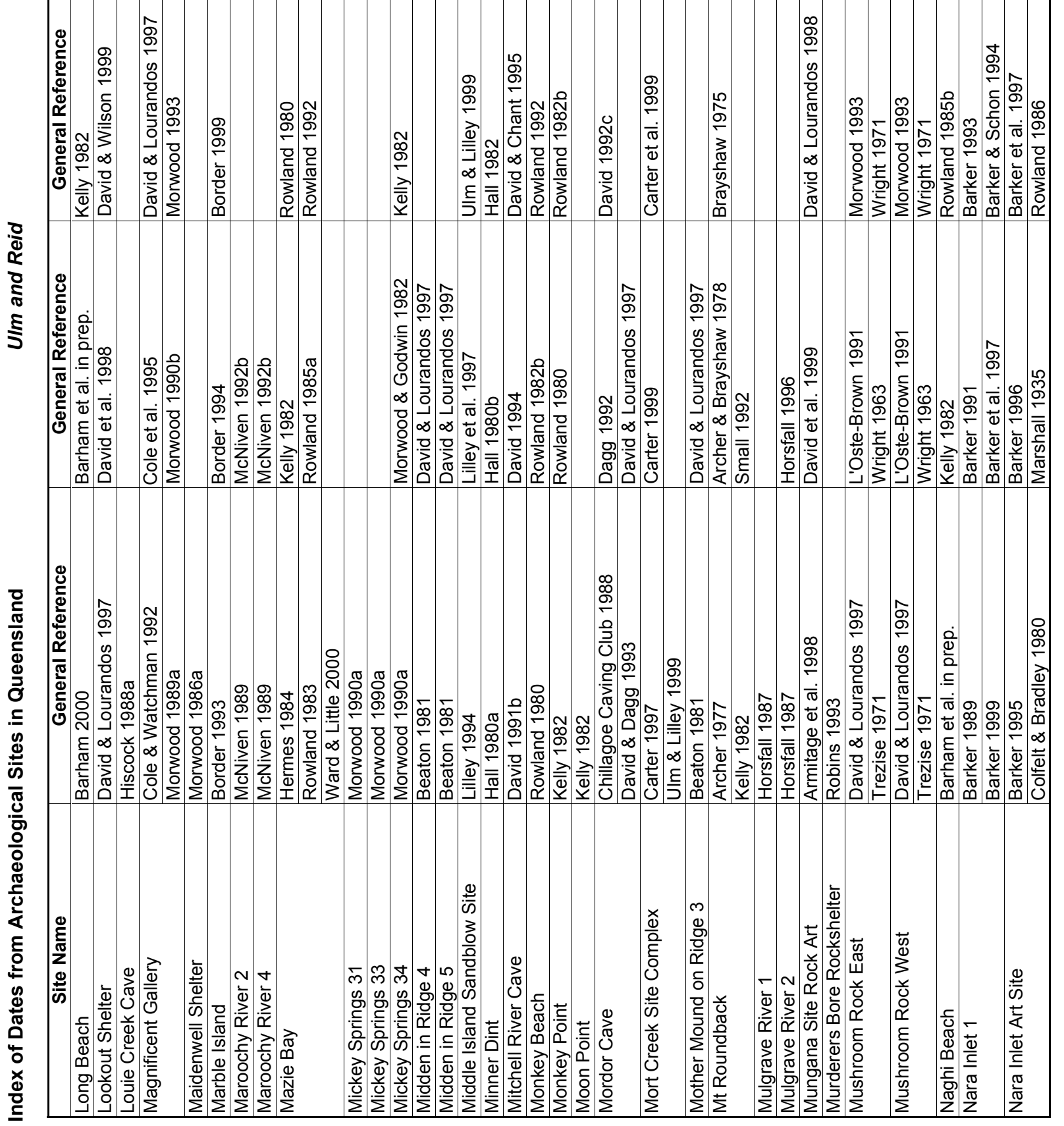

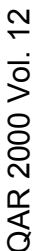



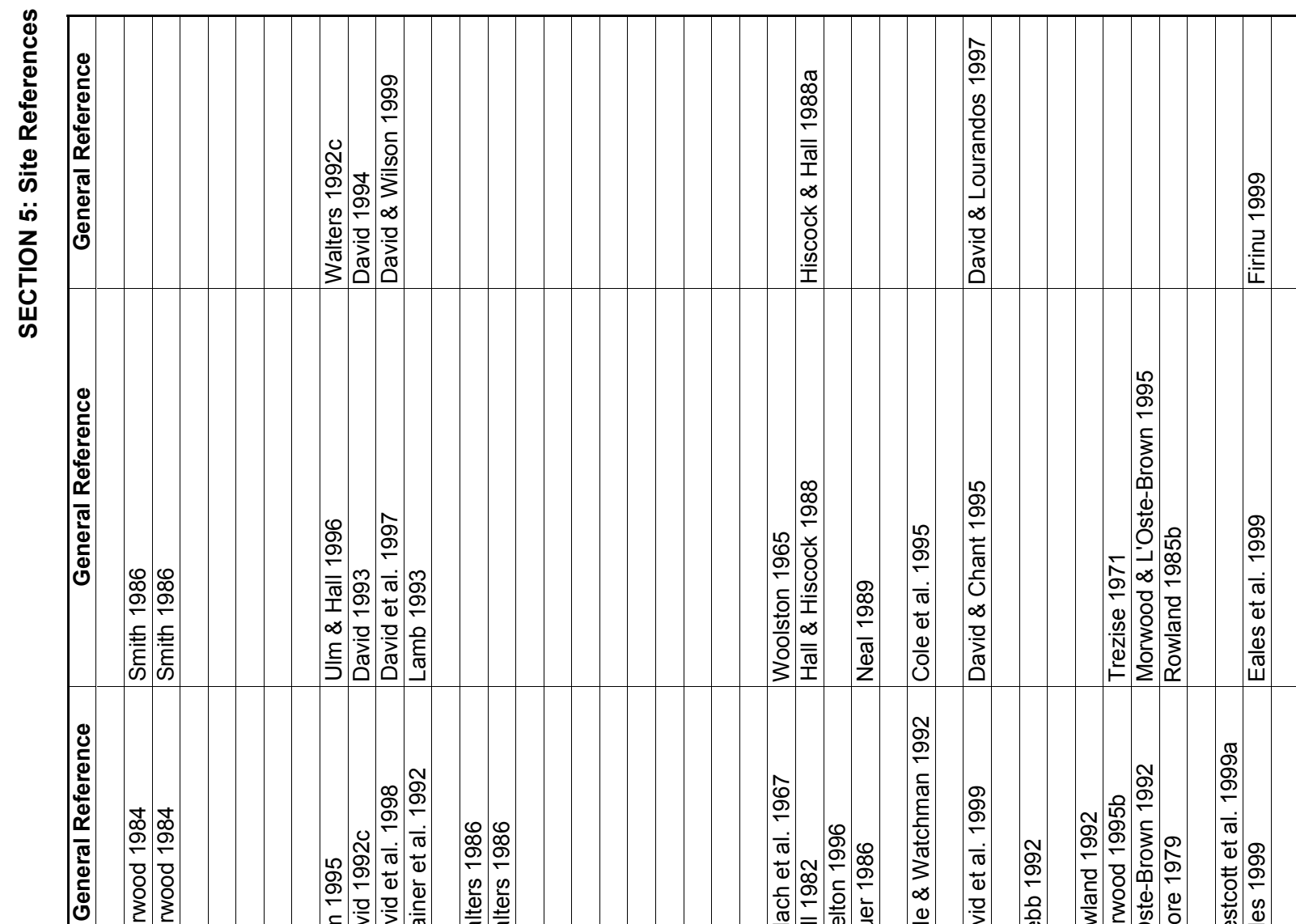

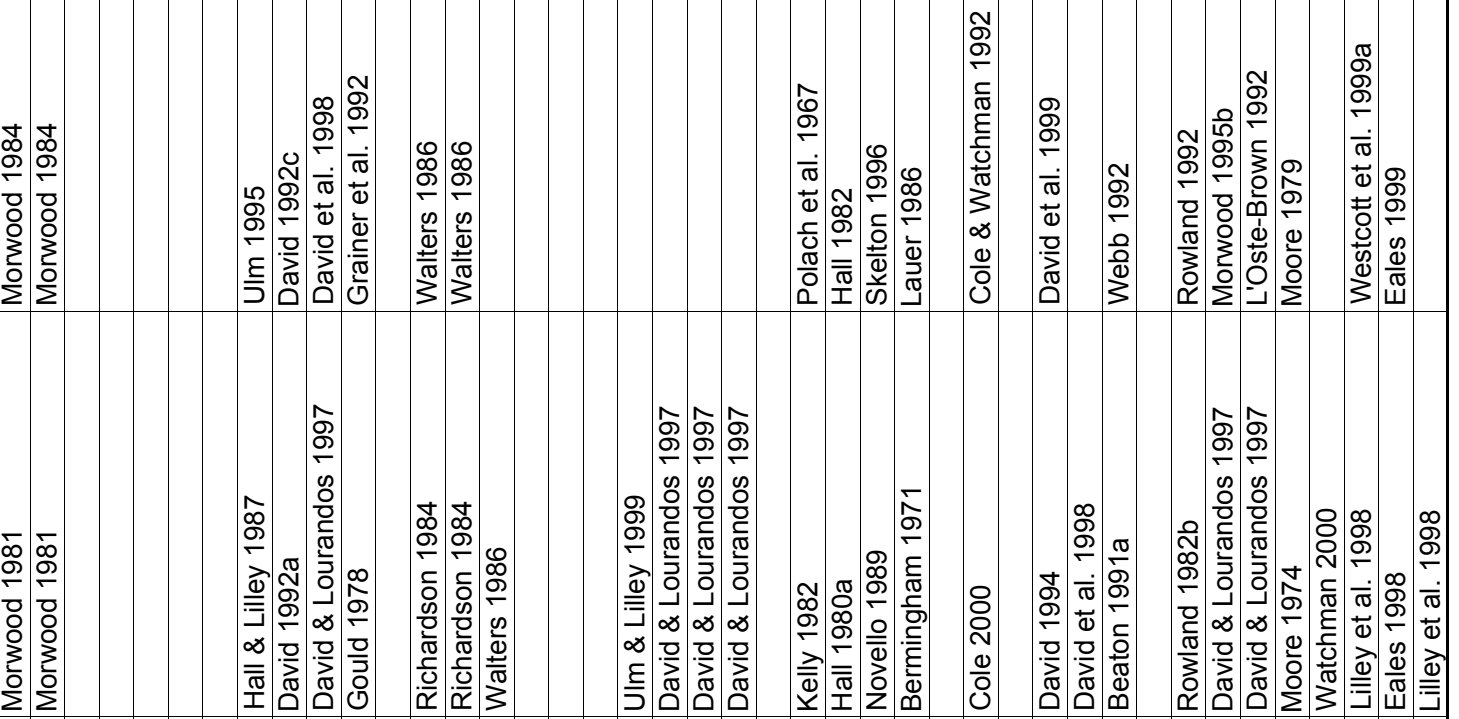

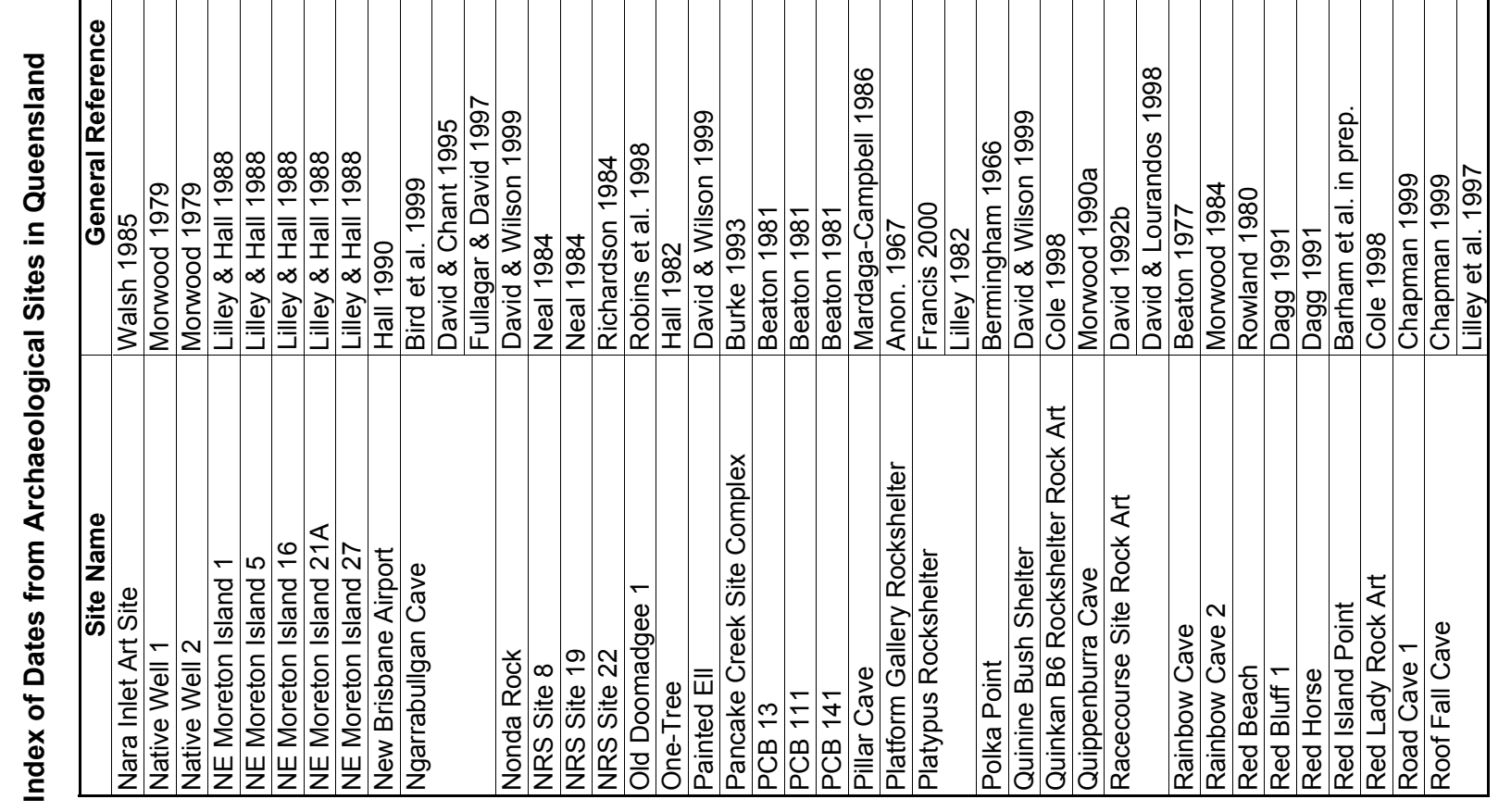



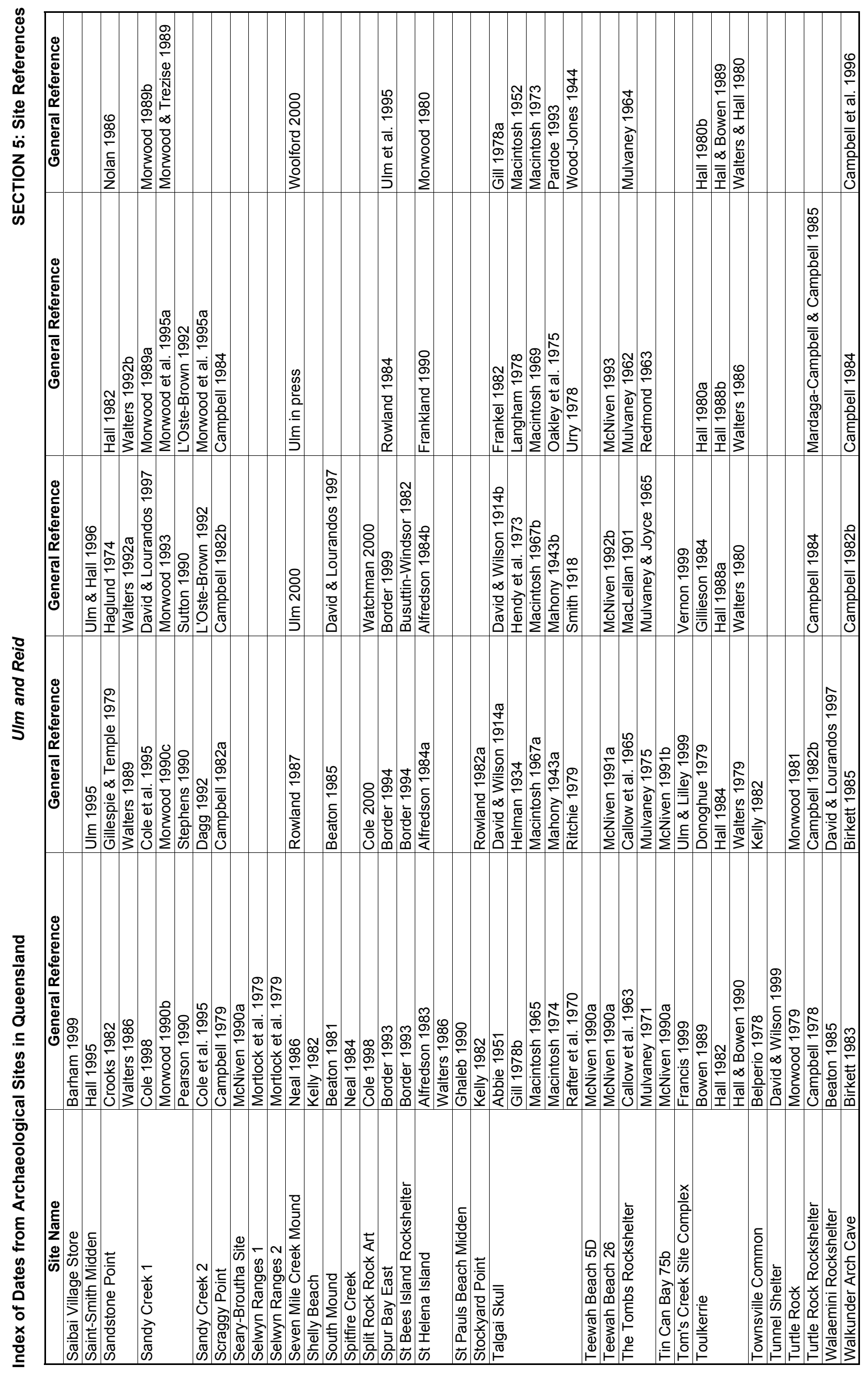


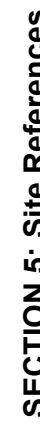

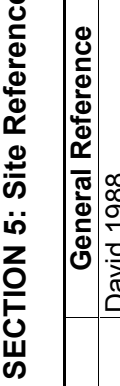

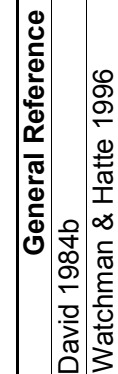

䟢

흘

选

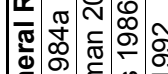

它

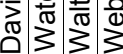

న్ల

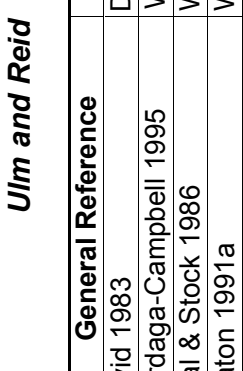

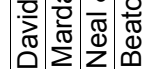

产

ஓ

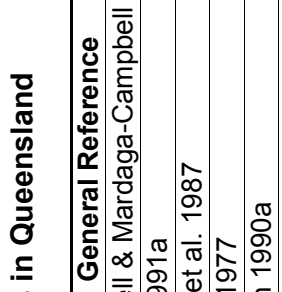

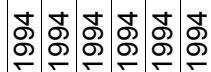

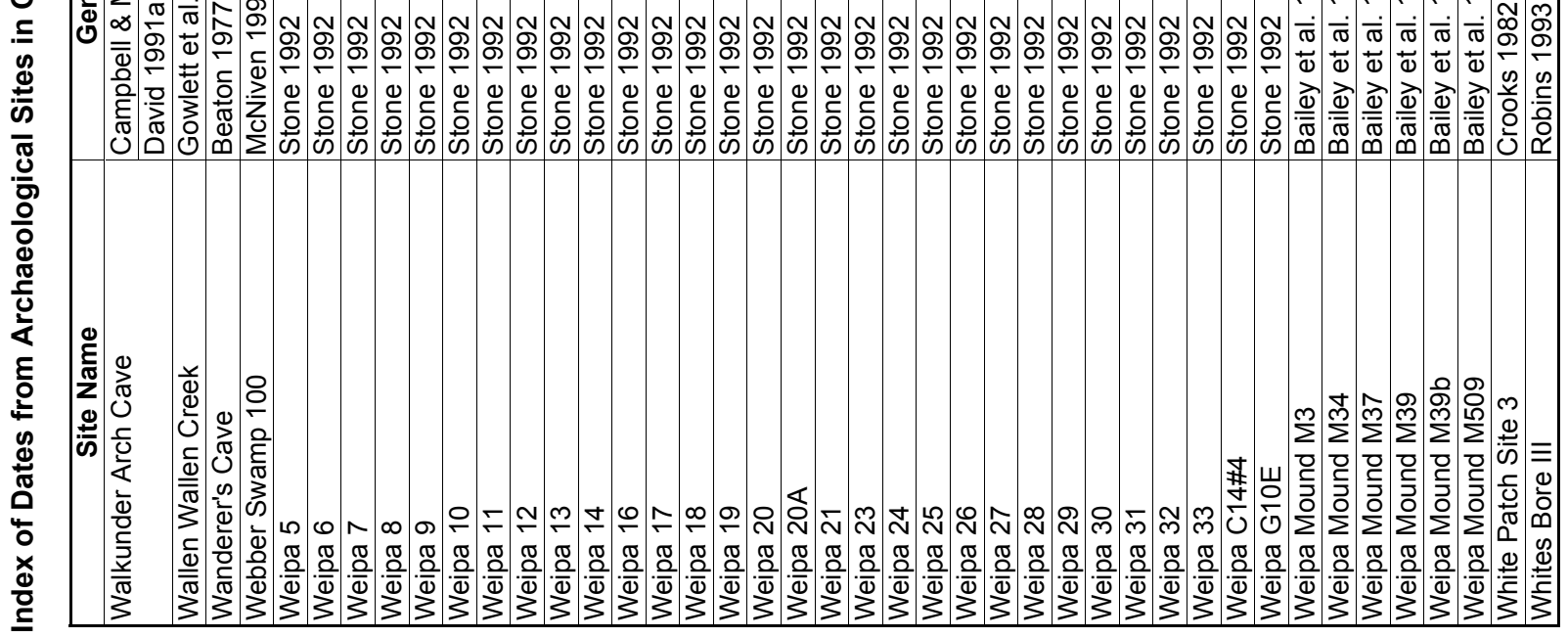

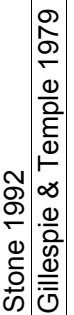

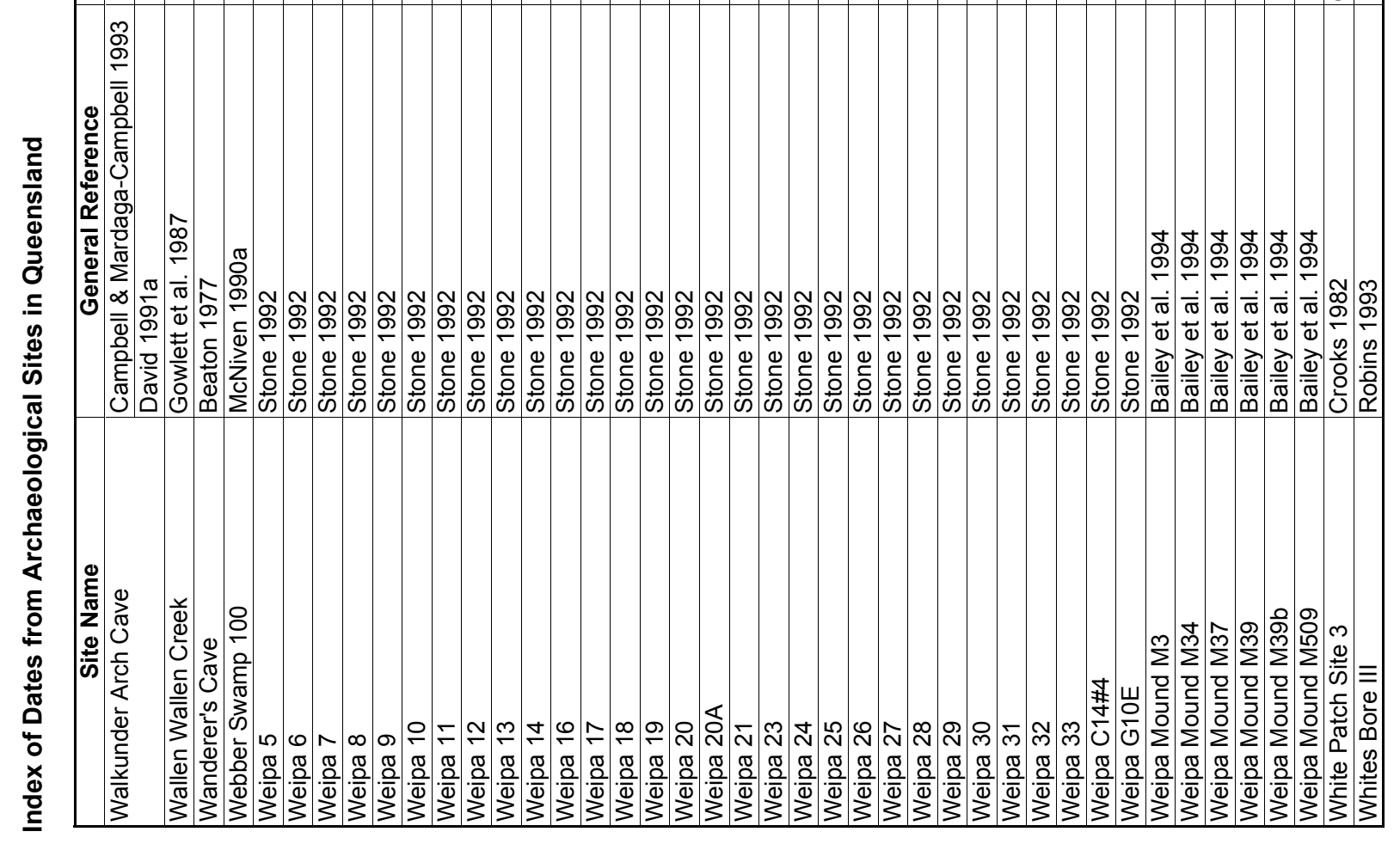

N गे \&

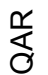




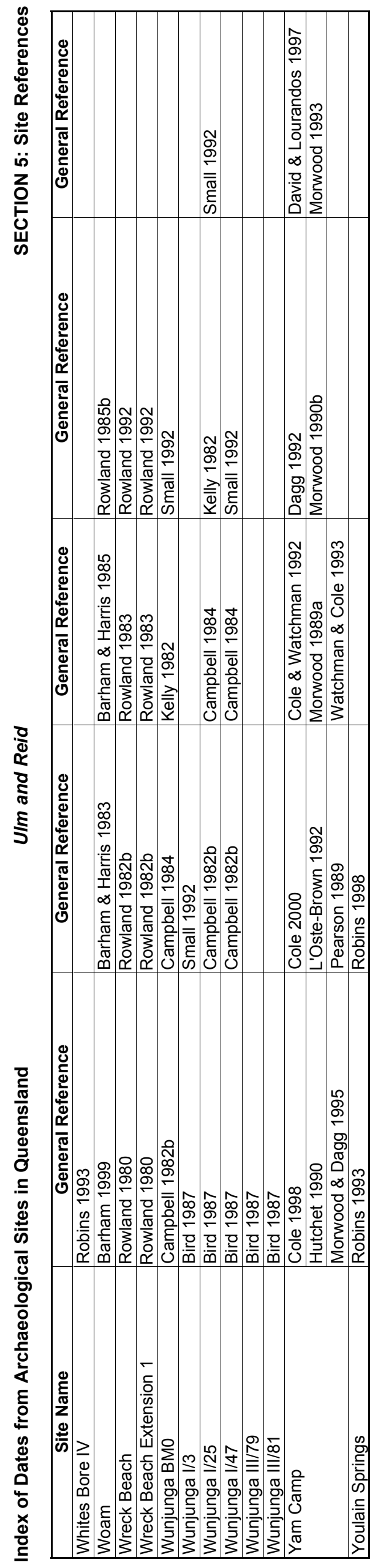




\section{SECTION 6:}

\section{Index of Sites by Principal Investigator}


SECTION 6: Index of Sites by Principal Investigator

\begin{tabular}{|c|c|c|c|}
\hline Investigator & Site Name & Investigator & Site Name \\
\hline \multirow[t]{2}{*}{ Alfredson, Gillian } & Lake Coombabah & Beaton, John & Weipa Mound M509 \\
\hline & St Helena Island & \multirow[t]{3}{*}{ Belperio, Antonio } & Gainsford 1 \\
\hline \multirow[t]{7}{*}{ Bailey, Geoff } & Kwamter Mound & & Gainsford 2 \\
\hline & Weipa Mound M3 & & Townsville Common \\
\hline & Weipa Mound M34 & \multirow[t]{4}{*}{ Bird, Michelle } & Wunjunga I/3 \\
\hline & Weipa Mound M37 & & Wunjunga I/25 \\
\hline & Weipa Mound M39 & & Wunjunga III/79 \\
\hline & Weipa Mound M39b & & Wunjunga III/81 \\
\hline & Weipa Mound M509 & \multirow[t]{7}{*}{ Border, Andrew } & Cape Palmerston \\
\hline \multirow[t]{6}{*}{ Barham, Anthony } & Bera Hill & & Castle Rock Cave \\
\hline & Gumu & & Curlew Island Open Site \\
\hline & Long Beach & & Curlew Island Rockshelter \\
\hline & Saibai Village Store & & Marble Island \\
\hline & St Pauls Beach Midden & & Spur Bay East \\
\hline & Woam & & St Bees Island Rockshelter \\
\hline \multirow[t]{4}{*}{ Barker, Bryce } & Border Island 1 & \multirow[t]{4}{*}{ Brayshaw, Helen } & Herveys Range B \\
\hline & Hill Inlet 1 & & Jourama Rockshelter \\
\hline & Nara Inlet 1 & & Kennedy A \\
\hline & Nara Inlet Art Site & & Mt Roundback \\
\hline \multirow[t]{44}{*}{ Beaton, John } & Alkaline Hill & \multirow[t]{9}{*}{ Campbell, John } & Jiyer Cave \\
\hline & Cathedral Cave & & Leefe Peak \\
\hline & Endaen Rockshelter & & Scraggy Point \\
\hline & Midden in Ridge 4 & & Shelly Beach \\
\hline & Midden in Ridge 5 & & Turtle Rock Rockshelter \\
\hline & Mother Mound on Ridge 3 & & Walkunder Arch Cave \\
\hline & PCB 13 & & Wunjunga BM0 \\
\hline & PCB 111 & & Wunjunga I/25 \\
\hline & PCB 141 & & Wunjunga I/47 \\
\hline & Rainbow Cave & \multirow[t]{7}{*}{ Cole, Noelene } & Deighton Lady Rock Art \\
\hline & Rainbow Cave 2 & & Kennedy River (Shelter B) Rock Art \\
\hline & South Mound & & Quinkan B6 Rockshelter Rock Art \\
\hline & Walaemini Rockshelter & & Red Lady Rock Art \\
\hline & Wanderer's Cave & & Sandy Creek 1 Rock Art \\
\hline & Weipa 5 & & Sandy Creek 2 Rock Art \\
\hline & Weipa 6 & & Split Rock Rock Art \\
\hline & Weipa 7 & \multirow[t]{2}{*}{ Conaghan, P.J. } & Deception Bay 1 \\
\hline & Weipa 8 & & Deception Bay 2 \\
\hline & Weipa 9 & \multirow[t]{25}{*}{ David, Bruno } & Bush Peg Shelter \\
\hline & Weipa 10 & & Chillagoe Site 55 Rock Art \\
\hline & Weipa 11 & & Chillagoe Site 62 Rock Art \\
\hline & Weipa 12 & & Chillagoe Site 75 Rock Art \\
\hline & Weipa 13 & & Courtyard Rock \\
\hline & Weipa 14 & & Dragonfly Hollow \\
\hline & Weipa 16 & & Echidna's Rest \\
\hline & Weipa 17 & & Fern Cave \\
\hline & Weipa 18 & & Fig Tree Shelter \\
\hline & Weipa 19 & & Gorge Creek Shelter \\
\hline & Weipa 20 & & Grass Tree Shelter \\
\hline & Weipa 20A & & Hand Shelter \\
\hline & Weipa 21 & & Hearth Cave \\
\hline & Weipa 23 & & Initiation Cave \\
\hline & Weipa 24 & & Kookaburra Rock \\
\hline & Weipa 25 & & Lookout Shelter \\
\hline & Weipa 26 & & Mitchell River Cave \\
\hline & Weipa 27 & & Mordor Cave \\
\hline & Weipa 28 & & Mungana Site Rock Art \\
\hline & Weipa 29 & & Ngarrabullgan Cave \\
\hline & Weipa 30 & & Nonda Rock \\
\hline & Weipa 31 & & Painted Ell \\
\hline & Weipa 32 & & Quinine Bush Shelter \\
\hline & Weipa 33 & & Racecourse Site Rock Art \\
\hline & Weipa C14\#4 & & Tunnel Shelter \\
\hline & Weipa G10E & Davidson, lain & Cuckadoo 1 \\
\hline
\end{tabular}


SECTION 6: Index of Sites by Principal Investigator

\begin{tabular}{|c|c|c|c|}
\hline Investigator & Site Name & Investigator & Site Name \\
\hline \multirow[t]{2}{*}{ Eales, Tony } & Grinding Groove Cave & \multirow[t]{4}{*}{ McNiven, Ian } & Teewah Beach 5D \\
\hline & Roof Fall Cave & & Teewah Beach 26 \\
\hline Edmonds, Vanessa & Bishop's Peak & & Tin Can Bay 75b \\
\hline \multirow[t]{2}{*}{ Flood, Josephine } & Echidna Shelter & & Webber Swamp 100 \\
\hline & Green Ant Shelter & Mills, Robynne & Freshwater Bay Midden \\
\hline \multirow[t]{3}{*}{ Flood, Peter } & Deception Bay 3 & \multirow[t]{3}{*}{ Moore, David } & Cape Cornwall \\
\hline & Deception Bay 4 & & Evans Bay \\
\hline & Deception Bay 5 & & Red Island Point \\
\hline Ghaleb, Barbara & Gumu & \multirow[t]{2}{*}{ Mortlock, A.J. } & Selwyn Ranges 1 \\
\hline Gill, Edmund & Talgai Skull & & Selwyn Ranges 2 \\
\hline Grimes, Ken & Moon Point & \multirow[t]{25}{*}{ Morwood, Mike } & Boonah Shelter \\
\hline \multirow[t]{3}{*}{ Haglund, Liala } & Broadbeach Burial Ground & & Gatton Shelter \\
\hline & Sandstone Point & & Giant Horse \\
\hline & White Patch Site 3 & & Goat Rock \\
\hline \multirow[t]{11}{*}{ Hall, Jay } & Bribie Island 9 & & Gyranda 5 \\
\hline & Brown's Road & & Gyranda 8 \\
\hline & Bushranger's Cave & & Gyranda 13 \\
\hline & Christmas Creek & & Gyranda 17 \\
\hline & \begin{tabular}{|l|} 
Minner Dint \\
\end{tabular} & & Ken's Cave \\
\hline & New Brisbane Airport & & Magnificent Gallery \\
\hline & One-Tree & & Maidenwell Shelter \\
\hline & Platypus Rockshelter & & Mickey Springs 31 \\
\hline & Saint-Smith Midden & & Mickey Springs 33 \\
\hline & Sandstone Point & & Mickey Springs 34 \\
\hline & Toulkerrie & & Mushroom Rock West \\
\hline \multirow[t]{4}{*}{ Hiscock, Peter } & Colless Creek Cave & & Mushroom Rock East \\
\hline & DH Site & & Native Well 1 \\
\hline & Lake Toomaroo & & Native Well 2 \\
\hline & Louie Creek Cave & & Quippenburra Cave \\
\hline Hopley, David & Abbott Bay & & Red Bluff 1 \\
\hline \multirow[t]{4}{*}{ Horsfall, Nicky } & Bramston Beach Midden & & Red Horse \\
\hline & Jiyer Cave & & Sandy Creek 1 \\
\hline & Mulgrave River 1 & & Sandy Creek 2 \\
\hline & Mulgrave River 2 & & Turtle Rock \\
\hline Hughes, Phil & Colless Creek Cave & & Yam Camp \\
\hline \multirow[t]{3}{*}{ Lauer, Peter } & Fraser Island 217/15 & \multirow[t]{2}{*}{ Mulvaney, John } & Kenniff Cave \\
\hline & Fraser Island 796/54 & & The Tombs Rockshelter \\
\hline & Fraser Island 799/54 & \multirow[t]{4}{*}{ Neal, Robert } & Aranarawai Beach Ridge II \\
\hline \multirow[t]{12}{*}{ Lilley, Ian } & Big Foot Art Site & & Polka Point \\
\hline & Boulder 1 & & Spitfire Creek \\
\hline & Eurimbula Site 1 & & Wallen Wallen Creek \\
\hline & Grinding Groove Cave & Richardson, Norma & NRS Site 8 \\
\hline & Mort Creek Site Complex & & NRS Site 19 \\
\hline & NE Moreton Island 1 & & NRS Site 22 \\
\hline & NE Moreton Island 5 & Robins, Richard & Bayley Point Mound 3 \\
\hline & NE Moreton Island 16 & & Cape Flattery \\
\hline & NE Moreton Island 21A & & First Ridge \\
\hline & NE Moreton Island 27 & & Gunamula \\
\hline & Road Cave 1 & & Kaponyee Springs I \\
\hline & Roof Fall Cave & & Kaponyee Springs II \\
\hline Lourandos, Harry & Hay Cave & & Kyeenee I \\
\hline Mardaga-Campbell, Miranda & Pillar Cave & & Lake Numalla I \\
\hline & Walkunder Arch Cave & & Lake Numalla II \\
\hline McNiven, Ian & Booral Homestead Midden & & Little Sandhills \\
\hline & Booral Shell Mound & & Murderers Bore Rockshelter \\
\hline & Brooyar Rockshelter & & Old Doomadgee 1 \\
\hline & Cameron Point 62 & & Whites Bore III \\
\hline & Double Island Point 1 & & Whites Bore IV \\
\hline & Kabali Road Scatter & & Youlain Springs \\
\hline & King's Bore Site 97 & Rosenfeld, Andree & Early Man Rockshelter \\
\hline & Maroochy River 2 & Rowland, Mike & Big Sandhills Beach \\
\hline & Maroochy River 4 & & Clam Bay \\
\hline & Seary-Broutha Site & & Long Beach \\
\hline
\end{tabular}


SECTION 6: Index of Sites by Principal Investigator

\begin{tabular}{|c|c|c|c|}
\hline Investigator & Site Name & Investigator & Site Name \\
\hline \multirow{9}{*}{ Rowland, Mike } & Mazie Bay & \multirow{6}{*}{ Ulm, Sean } & Ironbark Site Complex \\
\hline & Monkey Beach & & Middle Island Sandblow Site \\
\hline & Monkey Point & & Mort Creek Site Complex \\
\hline & Naghi Beach & & Pancake Creek Site Complex \\
\hline & Red Beach & & Seven Mile Creek Mound \\
\hline & Spur Bay East & & Tom's Creek Site Complex \\
\hline & Stockyard Point & \multirow[t]{2}{*}{ Walters, Ian } & Hope Island \\
\hline & Wreck Beach & & Sandstone Point \\
\hline & Wreck Beach Extension 1 & Ward, W.T. & Cribb Island \\
\hline Stone, Tim & Kwamter Mound & \multirow[t]{2}{*}{ Watchman, Alan } & Walkunder Arch Cave Rock Art \\
\hline Tugby, Donald & Polka Point & & Yam Camp Rock Art \\
\hline \multirow[t]{3}{*}{ Ulm, Sean } & Eurimbula Creek 1 & Woolston, F.P. & Platform Gallery Rockshelter \\
\hline & Eurimbula Creek 2 & \multirow[t]{2}{*}{ Wright, Richard } & Kwamter Mound \\
\hline & Eurimbula Site 1 & & Mushroom Rock West \\
\hline
\end{tabular}




\section{SECTION 7:}

\section{Index of Sites by Alternative Site Name}


SECTION 7: Index of Sites by Alternative Site Name

\begin{tabular}{|c|c|c|c|}
\hline Alternative Site Name & Site Name & Alternative Site Name & Site Name \\
\hline 9-Mile Shelter & Cathedral Cave & Jourama Rockshelter & Jourama Rockshelter \\
\hline Abbott Bay Shell Midden & Abbott Bay & Kennedy & Kennedy A \\
\hline Ash Cave & Initiation Cave & Kennedy Cave & Kennedy A \\
\hline \multirow[t]{2}{*}{ Beach Mount } & Wunjunga III/79 & Kennedy Rockshelter A & Kennedy A \\
\hline & Wunjunga III/81 & King's Bore Sandblow 97 & King's Bore Site 97 \\
\hline Beach Mount BM2 & Wunjunga I/25 & Laura Shelter & Mushroom Rock West \\
\hline Beach Mount BM2A & Wunjunga I/25 & Mickey Spring Gorge & Mickey Springs 34 \\
\hline Beach Mount BM4 & Wunjunga I/47 & Mount Roundback & Mt Roundback \\
\hline Beach Mount BMO & Wunjunga BM0 & \begin{tabular}{|l|} 
Mt Roundback Rockshelter \\
\end{tabular} & Mt Roundback \\
\hline Beach Mount I/3 & Wunjunga I/3 & Muralag & Cape Cornwall \\
\hline Booral Shell Midden & Booral Shell Mound & Murderers Bore 1 & Murderers Bore Rockshelter \\
\hline Bribie Island & White Patch Site 3 & Naghi Island & Naghi Beach \\
\hline Bribie Island Site 3 & White Patch Site 3 & Ngarrabullgan & Ngarrabullgan Cave \\
\hline Brown's Road Campsite & Brown's Road & Ngarrabullgin & Ngarrabullgan Cave \\
\hline CM56 & Echidna's Rest & Nullabullgin & Ngarrabullgan Cave \\
\hline Colless Creek Rockshelter & Colless Creek Cave & Nurrabullgin & Ngarrabullgan Cave \\
\hline Cooloola Site 97 & King's Bore Site 97 & Nurrabullgin 1 & Initiation Cave \\
\hline East Spur & Spur Bay East & Pigeon Creek Site & Gatton Shelter \\
\hline Embley River & Kwamter Mound & Port Lihou Bay & Cape Cornwall \\
\hline First Ridge 19b & First Ridge & Rocky Scrub Creek & Gatton Shelter \\
\hline Fraser Island, Moon Point & Moon Point & Rodds Peninsula Site Complex & Mort Creek Site Complex \\
\hline Goat Rock 1 & Goat Rock & Seegan & Long Beach \\
\hline Green Ant Rockshelter & Green Ant Shelter & St George River Shelter & Kennedy River (Shelter B) Rock Art \\
\hline Gumu 111 & Gumu & St Pauls Meach Midden 04 & St Pauls Beach Midden \\
\hline Gumu III & Gumu & The Tombs & The Tombs Rockshelter \\
\hline Hervey Range Rockshelter B & Herveys Range B & Toulkerrie Shell Midden & Toulkerrie \\
\hline Hervey's Range & Herveys Range B & Walaimini Rockshelter & Walaemini Rockshelter \\
\hline Hill Inlet Rock Site 1 & Hill Inlet 1 & Weipa Red Beach & Weipa Mound M509 \\
\hline Jiger Cave & Jiyer Cave & Weipa Shell Midden & Kwamter Mound \\
\hline
\end{tabular}




\section{SECTION 8:}

\section{Index of Sites by Conventional Radiocarbon Age (TL \& OSL dates excluded)}


SECTION 8: Index of Sites by Conventional Radiocarbon Age

\begin{tabular}{|c|c|c|c|c|c|}
\hline CRA & \pm & Site Name & CRA & \pm & Site Name \\
\hline$>37170$ & NA & Ngarrabullgan Cave & 16100 & 130 & Walkunder Arch Cave Rock Art \\
\hline 36100 & 800 & Ngarrabullgan Cave & 15950 & 770 & Walkunder Arch Cave \\
\hline 35500 & 600 & Ngarrabullgan Cave & 15910 & 200 & Mitchell River Cave \\
\hline 35460 & $+750 /-690$ & Ngarrabullgan Cave & 15900 & 260 & Kenniff Cave \\
\hline 35200 & $+630 /-580$ & Ngarrabullgan Cave & 15820 & 190 & Colless Creek Cave \\
\hline 35200 & $+720 /-660$ & Ngarrabullgan Cave & 15720 & 380 & Roof Fall Cave \\
\hline 34600 & 350 & Ngarrabullgan Cave & 15450 & 1500 & Early Man Rockshelter \\
\hline 34580 & $+580 /-540$ & Ngarrabullgan Cave & 15270 & 210 & Cuckadoo 1 \\
\hline 34350 & $+720 /-660$ & Ngarrabullgan Cave & 14980 & 235 & Kenniff Cave \\
\hline 34230 & $+600 /-560$ & Ngarrabullgan Cave & 14210 & 230 & Louie Creek Cave \\
\hline 34200 & 300 & Ngarrabullgan Cave & 14150 & 160 & Colless Creek Cave \\
\hline 33630 & $+480 /-450$ & Ngarrabullgan Cave & 14150 & 255 & Kenniff Cave \\
\hline 33470 & $+580 /-540$ & Ngarrabullgan Cave & 14140 & 225 & Kenniff Cave \\
\hline 33420 & $+490 /-460$ & Ngarrabullgan Cave & 13830 & 630 & Youlain Springs \\
\hline 33400 & 3750 & Ngarrabullgan Cave & 13810 & 260 & Youlain Springs \\
\hline 33320 & $+480 /-450$ & Ngarrabullgan Cave & 13710 & 260 & Roof Fall Cave \\
\hline 33200 & 450 & Ngarrabullgan Cave & 13650 & 240 & Wallen Wallen Creek \\
\hline 33170 & $+570 /-530$ & Ngarrabullgan Cave & 13620 & 160 & Colless Creek Cave \\
\hline 33100 & 550 & Ngarrabullgan Cave & 13500 & 400 & Wallen Wallen Creek \\
\hline 33100 & 2100 & Ngarrabullgan Cave & 13490 & 110 & Walkunder Arch Cave \\
\hline 33000 & 450 & Ngarrabullgan Cave & 13230 & 440 & Youlain Springs \\
\hline 33000 & 450 & Ngarrabullgan Cave & 13200 & 170 & Early Man Rockshelter \\
\hline 32900 & 550 & Ngarrabullgan Cave & 13200 & 300 & Kenniff Cave \\
\hline 32700 & 450 & Ngarrabullgan Cave & 13150 & 120 & Walkunder Arch Cave \\
\hline 32540 & $+630 /-590$ & Ngarrabullgan Cave & 13040 & 220 & Wallen Wallen Creek \\
\hline 32400 & 400 & Ngarrabullgan Cave & 12900 & 170 & Kenniff Cave \\
\hline 32300 & 500 & Ngarrabullgan Cave & 12840 & 100 & Walkunder Arch Cave Rock Art \\
\hline 32200 & 500 & Ngarrabullgan Cave & 12820 & 230 & Youlain Springs \\
\hline 31900 & $+700 /-600$ & Sandy Creek 1 & 12780 & 90 & Walkunder Arch Cave \\
\hline 31800 & 1650 & Ngarrabullgan Cave & 12620 & 370 & Sandy Creek 1 \\
\hline 31510 & $+490 /-460$ & Ngarrabullgan Cave & 12610 & 110 & Kenniff Cave \\
\hline 31300 & 1900 & Ngarrabullgan Cave & 12600 & 2800 & Early Man Rockshelter \\
\hline 31100 & 400 & Ngarrabullgan Cave & 12430 & 95 & Cuckadoo 1 \\
\hline 30960 & $+550 /-520$ & Ngarrabullgan Cave & 12400 & 300 & Talgai Skull \\
\hline 30700 & 250 & Ngarrabullgan Cave & 12250 & 80 & Cuckadoo 1 \\
\hline 30300 & 800 & Fern Cave & 12140 & 150 & Kenniff Cave \\
\hline 30000 & 600 & Ngarrabullgan Cave & 12120 & 510 & Cuckadoo 1 \\
\hline 29700 & 500 & Walkunder Arch Cave Rock Art & 11990 & 70 & Wallen Wallen Creek \\
\hline 28800 & 1100 & Ngarrabullgan Cave & 11870 & 110 & Walkunder Arch Cave \\
\hline 28100 & 400 & Walkunder Arch Cave Rock Art & 11850 & 210 & Early Man Rockshelter \\
\hline 26010 & 410 & Fern Cave & 11650 & 100 & Talgai Skull \\
\hline 25800 & 280 & Walkunder Arch Cave Rock Art & 11090 & 235 & Kenniff Cave \\
\hline 25710 & 490 & Fern Cave & 10910 & 140 & Native Well 1 \\
\hline 24600 & 220 & Sandy Creek 2 Rock Art & 10770 & 135 & Native Well 2 \\
\hline 22800 & 210 & Walkunder Arch Cave Rock Art & 10400 & 90 & Walkunder Arch Cave Rock Art \\
\hline 21800 & 400 & Wallen Wallen Creek & 10280 & 180 & Kenniff Cave \\
\hline 21500 & 250 & Hearth Cave & 10250 & 90 & Magnificent Gallery \\
\hline 21430 & 400 & Wallen Wallen Creek & 10240 & 420 & Walkunder Arch Cave \\
\hline 20890 & 260 & Fern Cave & 10200 & 300 & Broadbeach Burial Ground \\
\hline 20800 & 160 & Walkunder Arch Cave Rock Art & 10200 & 210 & Fern Cave \\
\hline 20560 & 250 & Wallen Wallen Creek & 10120 & 60 & Tunnel Shelter \\
\hline 19520 & 170 & Walkunder Arch Cave & 9920 & 250 & Mickey Springs 34 \\
\hline 18800 & 480 & Kenniff Cave & 9810 & 130 & Wallen Wallen Creek \\
\hline 18680 & 180 & Walkunder Arch Cave & 9800 & 220 & The Tombs Rockshelter \\
\hline 18600 & $+900 /-800$ & Louie Creek Cave & 9790 & 60 & Talgai Skull \\
\hline 18200 & 450 & Early Man Rockshelter & 9760 & 140 & Wallen Wallen Creek \\
\hline 17500 & $+900 /-800$ & Wallen Wallen Creek & 9650 & 100 & Kenniff Cave \\
\hline 17290 & 470 & Colless Creek Cave & 9640 & 120 & Wallen Wallen Creek \\
\hline 17200 & 100 & Fern Cave & 9590 & 490 & Talgai Skull \\
\hline 16900 & 280 & Yam Camp & 9530 & 335 & Talgai Skull \\
\hline 16420 & 260 & Wallen Wallen Creek & 9470 & 120 & Walkunder Arch Cave Rock Art \\
\hline 16170 & 260 & Colless Creek Cave & 9410 & 100 & The Tombs Rockshelter \\
\hline 16130 & 140 & Kenniff Cave & 9300 & 200 & Kenniff Cave \\
\hline
\end{tabular}


SECTION 8: Index of Sites by Conventional Radiocarbon Age

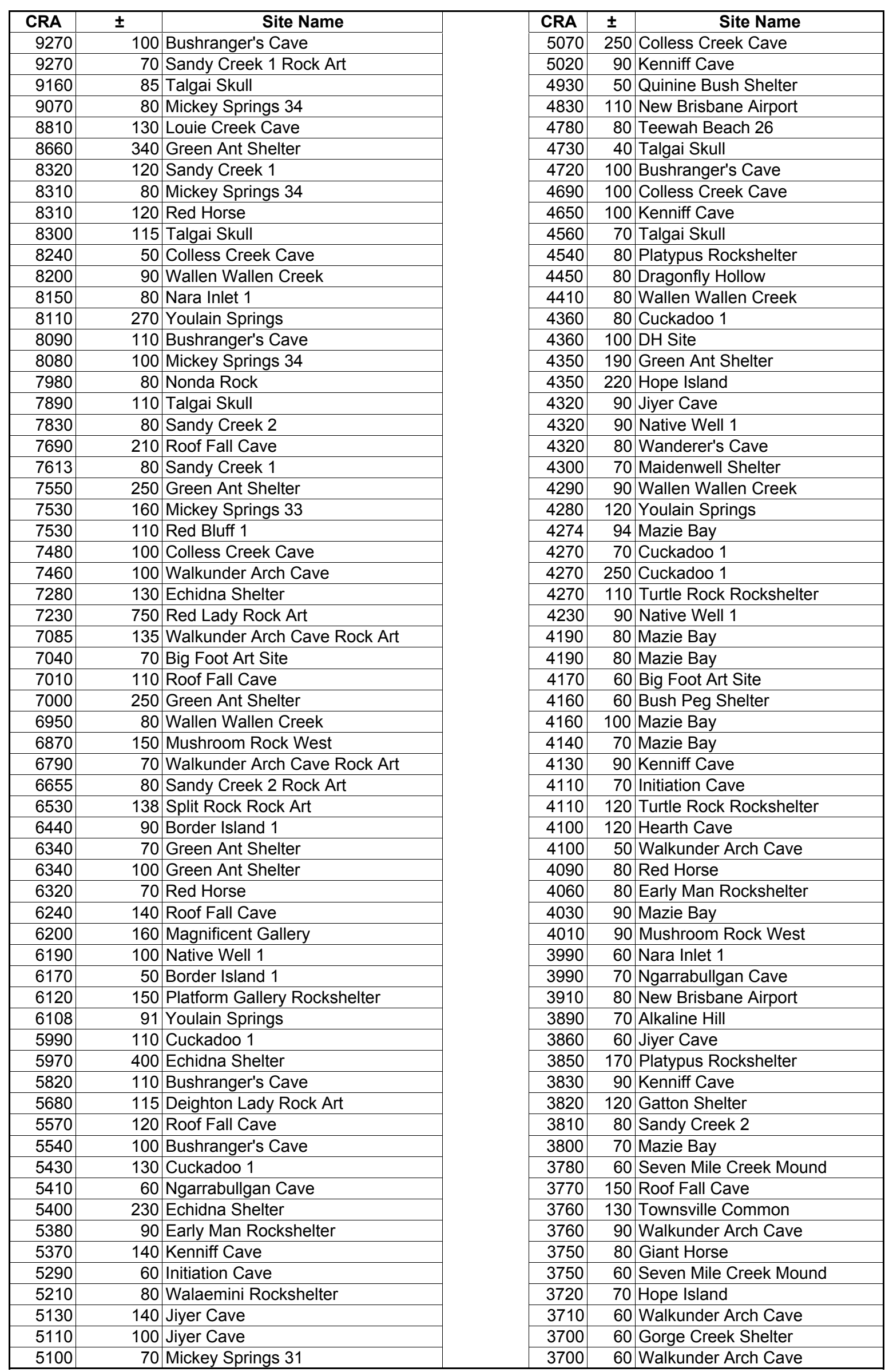


SECTION 8: Index of Sites by Conventional Radiocarbon Age

\begin{tabular}{|c|c|c|c|c|c|}
\hline CRA & \pm & Site Name & CRA & \pm & Site Name \\
\hline 3670 & 100 & Walkunder Arch Cave & 2820 & 60 & Walkunder Arch Cave \\
\hline 3660 & 80 & Jiyer Cave & 2810 & 150 & Sandy Creek 1 Rock Art \\
\hline 3660 & 60 & Seven Mile Creek Mound & 2800 & 80 & Courtyard Rock \\
\hline 3610 & 70 & Seven Mile Creek Mound & 2800 & 300 & Turtle Rock \\
\hline 3600 & 70 & Mazie Bay & 2790 & 80 & Booral Shell Mound \\
\hline 3600 & 100 & Rainbow Cave & 2784 & 85 & Deighton Lady Rock Art \\
\hline 3600 & 93 & The Tombs Rockshelter & 2770 & 110 & Eurimbula Site 1 \\
\hline 3570 & 60 & Seven Mile Creek Mound & 2750 & 80 & Rainbow Cave 2 \\
\hline 3560 & 80 & Cathedral Cave & 2740 & 70 & Ngarrabullgan Cave \\
\hline 3560 & 100 & King's Bore Site 97 & 2740 & 70 & Spur Bay East \\
\hline 3550 & 70 & Walkunder Arch Cave & 2720 & 120 & Hill Inlet 1 \\
\hline 3540 & 80 & Seven Mile Creek Mound & 2720 & 60 & Mort Creek Site Complex \\
\hline 3500 & 60 & Seven Mile Creek Mound & 2700 & 110 & Weipa Mound M34 \\
\hline 3494 & 84 & Hearth Cave & 2690 & 100 & Mulgrave River 2 \\
\hline 3480 & 200 & Grinding Groove Cave & 2686 & 69 & Grinding Groove Cave \\
\hline 3460 & 80 & Bushranger's Cave & 2680 & 60 & Mort Creek Site Complex \\
\hline 3450 & 70 & Mazie Bay & 2680 & 90 & Walkunder Arch Cave \\
\hline 3430 & 140 & Mort Creek Site Complex & 2670 & 290 & Chillagoe Site 75 Rock Art \\
\hline 3420 & 90 & Walkunder Arch Cave & 2670 & 70 & Walkunder Arch Cave \\
\hline 3410 & 60 & Walkunder Arch Cave & 2660 & 60 & Booral Shell Mound \\
\hline 3400 & 170 & Colless Creek Cave & 2650 & 60 & Brooyar Rockshelter \\
\hline 3400 & 80 & Sandy Creek 1 & 2650 & 160 & Jiyer Cave \\
\hline 3400 & 97 & The Tombs Rockshelter & 2620 & 90 & Bishop's Peak \\
\hline 3400 & 90 & Turtle Rock Rockshelter & 2600 & 70 & Hope Island \\
\hline 3380 & 90 & Mort Creek Site Complex & 2600 & 60 & Walkunder Arch Cave \\
\hline 3370 & 130 & Colless Creek Cave & 2570 & 60 & Mort Creek Site Complex \\
\hline 3360 & 60 & Mickey Springs 34 & 2550 & 90 & Kenniff Cave \\
\hline 3350 & 350 & Mungana Site Rock Art & 2530 & 60 & Walkunder Arch Cave \\
\hline 3340 & 80 & Early Man Rockshelter & 2510 & 50 & Boonah Shelter \\
\hline 3340 & 60 & Walkunder Arch Cave Rock Art & 2510 & 130 & Spur Bay East \\
\hline 3330 & 80 & Cathedral Cave & 2500 & 60 & Lake Coombabah \\
\hline 3330 & 440 & Chillagoe Site 55 Rock Art & 2480 & 60 & Booral Shell Mound \\
\hline 3290 & 60 & Boulder 1 & 2480 & 70 & Platypus Rockshelter \\
\hline 3280 & 80 & Bribie Island 9 & 2470 & 80 & Native Well 2 \\
\hline 3280 & 100 & Quippenburra Cave & 2450 & 230 & Green Ant Shelter \\
\hline 3270 & 60 & Christmas Creek & 2440 & 150 & Echidna's Rest \\
\hline 3260 & 110 & Border Island 1 & 2440 & 120 & Mitchell River Cave \\
\hline 3260 & 70 & Mort Creek Site Complex & 2440 & 80 & Mort Creek Site Complex \\
\hline 3240 & 50 & Boonah Shelter & 2440 & 60 & Ngarrabullgan Cave \\
\hline 3180 & 150 & St Bees Island Rockshelter & 2420 & 80 & Booral Homestead Midden \\
\hline 3140 & 100 & Teewah Beach 26 & 2420 & 90 & Platypus Rockshelter \\
\hline 3130 & 70 & Mazie Bay & 2420 & 100 & Walkunder Arch Cave \\
\hline 3090 & 70 & Cuckadoo 1 & 2410 & 50 & Initiation Cave \\
\hline 3050 & 160 & Grinding Groove Cave & 2410 & 80 & Nara Inlet Art Site \\
\hline 3040 & 60 & Walkunder Arch Cave & 2410 & 80 & Turtle Rock \\
\hline 3030 & 90 & Gatton Shelter & 2410 & 70 & Walkunder Arch Cave \\
\hline 3020 & 70 & Eurimbula Site 1 & 2403 & 90 & Walkunder Arch Cave \\
\hline 3020 & 100 & Polka Point & 2400 & 110 & Booral Homestead Midden \\
\hline 3000 & 80 & Dragonfly Hollow & 2390 & 60 & Eurimbula Site 1 \\
\hline 2970 & 80 & Freshwater Bay Midden & 2390 & 70 & Eurimbula Site 1 \\
\hline 2960 & 80 & Cathedral Cave & 2380 & 90 & South Mound \\
\hline 2960 & 80 & Kookaburra Rock & 2380 & 70 & Walkunder Arch Cave \\
\hline 2950 & 60 & Booral Shell Mound & 2380 & 70 & Walkunder Arch Cave \\
\hline 2940 & 110 & Colless Creek Cave & 2360 & 70 & Hearth Cave \\
\hline 2930 & 120 & Curlew Island Rockshelter & 2360 & 60 & Walkunder Arch Cave \\
\hline 2930 & 80 & Grinding Groove Cave & 2350 & 70 & Cape Palmerston \\
\hline 2930 & 60 & Mort Creek Site Complex & 2350 & 100 & Mushroom Rock East \\
\hline 2890 & 60 & Woam & 2320 & 50 & Mort Creek Site Complex \\
\hline 2880 & 50 & Mort Creek Site Complex & 2320 & 60 & Red Bluff 1 \\
\hline 2860 & 60 & Road Cave 1 & 2300 & 90 & Castle Rock Cave \\
\hline 2850 & 80 & Early Man Rockshelter & 2300 & 70 & Cathedral Cave \\
\hline 2850 & 115 & Quinkan B6 Rockshelter Rock Art & 2290 & 80 & King's Bore Site 97 \\
\hline 2820 & 90 & Endaen Rockshelter & 2290 & 100 & Sandstone Point \\
\hline
\end{tabular}


SECTION 8: Index of Sites by Conventional Radiocarbon Age

\begin{tabular}{|c|c|c|c|c|c|}
\hline CRA & \pm & Site Name & CRA & \pm & Site Name \\
\hline 2290 & 80 & Toulkerrie & 1770 & 70 & Mulgrave River 2 \\
\hline 2280 & 190 & Long Beach & 1750 & 60 & Booral Shell Mound \\
\hline 2260 & 50 & Mort Creek Site Complex & 1750 & 90 & Polka Point \\
\hline 2240 & 70 & St Helena Island & 1740 & 70 & Gunamula \\
\hline 2230 & 70 & South Mound & 1740 & 50 & Lake Toomaroo \\
\hline 2170 & 80 & Native Well 2 & 1730 & 80 & Whites Bore III \\
\hline 2170 & 80 & Walkunder Arch Cave & 1725 & 80 & PCB 141 \\
\hline 2165 & 55 & South Mound & 1690 & 60 & Cuckadoo 1 \\
\hline 2165 & 55 & South Mound & 1690 & 90 & Leefe Peak \\
\hline 2160 & 60 & Jiyer Cave & 1690 & 70 & South Mound \\
\hline 2120 & 80 & South Mound & 1630 & 80 & Kookaburra Rock \\
\hline 2120 & 70 & Wallen Wallen Creek & 1620 & 60 & One-Tree \\
\hline 2110 & 80 & South Mound & 1620 & 70 & South Mound \\
\hline 2100 & 80 & Weipa 20 & 1600 & 160 & Eurimbula Site 1 \\
\hline 2090 & 90 & Bushranger's Cave & 1600 & 100 & Kenniff Cave \\
\hline 2090 & 50 & Nara Inlet 1 & 1600 & 45 & Lookout Shelter \\
\hline 2090 & 90 & South Mound & 1600 & 80 & Sandstone Point \\
\hline 2080 & 100 & Chillagoe Site 55 Rock Art & 1600 & 45 & South Mound \\
\hline 2070 & 60 & Weipa 18 & 1600 & 70 & South Mound \\
\hline 2030 & 70 & South Mound & 1560 & 80 & Polka Point \\
\hline 2030 & 80 & South Mound & 1560 & 60 & Weipa Mound M39b \\
\hline 2010 & 70 & South Mound & 1550 & 60 & Ngarrabullgan Cave \\
\hline 2010 & 80 & Weipa 21 & 1540 & 60 & Bayley Point Mound 3 \\
\hline 2000 & 510 & Chillagoe Site 75 Rock Art & 1530 & 70 & Walkunder Arch Cave \\
\hline 2000 & 80 & Ken's Cave & 1520 & 80 & Gyranda 17 \\
\hline 1990 & 90 & Christmas Creek & 1520 & 50 & Mazie Bay \\
\hline 1990 & 60 & Sandstone Point & 1520 & 70 & Walkunder Arch Cave \\
\hline 1980 & 70 & Bushranger's Cave & 1520 & 70 & Wallen Wallen Creek \\
\hline 1980 & 70 & South Mound & 1520 & 80 & Weipa 28 \\
\hline 1970 & 80 & Mort Creek Site Complex & 1510 & 170 & Deception Bay 1 \\
\hline 1965 & 100 & Fraser Island 796/54 & 1500 & 80 & Hope Island \\
\hline 1960 & 110 & Boonah Shelter & 1500 & 110 & Sandstone Point \\
\hline 1960 & 110 & Fraser Island 217/15 & 1500 & 70 & South Mound \\
\hline 1940 & 80 & Pillar Cave & 1500 & 70 & South Mound \\
\hline 1940 & 90 & Weipa Mound M37 & 1500 & 90 & South Mound \\
\hline 1920 & 70 & South Mound & 1470 & 170 & Echidna's Rest \\
\hline 1920 & 140 & Walkunder Arch Cave & 1470 & 80 & Moon Point \\
\hline 1910 & 70 & South Mound & 1460 & 60 & Weipa 12 \\
\hline 1890 & 70 & Sandy Creek 1 & 1455 & 140 & Herveys Range B \\
\hline
\end{tabular}


SECTION 8: Index of Sites by Conventional Radiocarbon Age

\begin{tabular}{|c|c|c|c|c|c|}
\hline CRA & \pm & Site Name & CRA & \pm & Site Name \\
\hline 1390 & 70 & Midden in Ridge 5 & 1070 & 50 & Wallen Wallen Creek \\
\hline 1390 & 80 & Weipa 26 & 1060 & 80 & Magnificent Gallery \\
\hline 1380 & 70 & Ken's Cave & 1060 & 130 & Weipa Mound M509 \\
\hline 1370 & 50 & Quippenburra Cave & 1050 & 50 & Grass Tree Shelter \\
\hline 1370 & 80 & South Mound & 1050 & 100 & Gumu \\
\hline 1370 & 60 & St Helena Island & 1050 & 50 & Gyranda 8 \\
\hline 1360 & 50 & Ngarrabullgan Cave & 1050 & 70 & PCB 141 \\
\hline 1340 & 40 & Marble Island & 1040 & 60 & Road Cave 1 \\
\hline 1340 & 50 & South Mound & 1030 & 40 & Kwamter Mound \\
\hline 1330 & 60 & Walkunder Arch Cave & 1020 & 70 & Red Beach \\
\hline 1330 & 80 & Weipa 29 & 1010 & 50 & Ngarrabullgan Cave \\
\hline 1310 & 460 & Echidna's Rest Rock Art & 1000 & 65 & Bayley Point Mound 3 \\
\hline 1300 & 70 & Bayley Point Mound 3 & 1000 & 120 & Chillagoe Site 55 Rock Art \\
\hline 1300 & 60 & Boonah Shelter & 1000 & 70 & Christmas Creek \\
\hline 1300 & 90 & Cribb Island & 1000 & 140 & Deception Bay 1 \\
\hline 1300 & 100 & South Mound & 995 & 70 & Deception Bay 3 \\
\hline 1290 & 60 & Pillar Cave & 990 & 485 & Chillagoe Site 62 Rock Art \\
\hline 1280 & 70 & Goat Rock & 990 & 70 & Kwamter Mound \\
\hline 1280 & 70 & Kyeenee I & 980 & 60 & Booral Shell Mound \\
\hline 1280 & 50 & Ngarrabullgan Cave & 980 & 40 & Kwamter Mound \\
\hline 1270 & 250 & Chillagoe Site 62 Rock Art & 980 & 50 & Middle Island Sandblow Site \\
\hline 1270 & 80 & Fraser Island 799/54 & 970 & 50 & Ngarrabullgan Cave \\
\hline 1270 & 70 & Native Well 1 & 970 & 60 & Weipa 14 \\
\hline 1260 & 80 & Seven Mile Creek Mound & 960 & 80 & Roof Fall Cave \\
\hline 1250 & 80 & Weipa 6 & 960 & 60 & Weipa 31 \\
\hline 1230 & 60 & Ngarrabullgan Cave & 950 & 60 & Cameron Point 62 \\
\hline 1230 & 50 & Sandy Creek 1 & 950 & 70 & Early Man Rockshelter \\
\hline 1230 & 50 & Wallen Wallen Creek & 950 & 50 & Painted Ell \\
\hline 1220 & 50 & Road Cave 1 & 950 & 80 & Red Beach \\
\hline 1210 & 245 & Kennedy River (Shelter B) Rock Art & 950 & 100 & Teewah Beach 26 \\
\hline 1210 & 100 & Maidenwell Shelter & 950 & 50 & Yam Camp \\
\hline 1210 & 60 & Weipa 8 & 940 & 60 & Mordor Cave \\
\hline 1200 & 440 & Mungana Site Rock Art & 940 & 50 & Tom's Creek Site Complex \\
\hline 1190 & 100 & Broadbeach Burial Ground & 930 & 80 & Kwamter Mound \\
\hline 1190 & 90 & Midden in Ridge 5 & 930 & 70 & Mitchell River Cave \\
\hline 1190 & 100 & Sandstone Point & 930 & 55 & Ngarrabullgan Cave \\
\hline 1180 & 110 & Broadbeach Burial Ground & 930 & 60 & Saint-Smith Midden \\
\hline 1180 & 80 & Kwamter Mound & 910 & 55 & Ironbark Site Complex \\
\hline 1180 & 70 & Road Cave 1 & 910 & 90 & Kwamter Mound \\
\hline 1170 & 50 & Cuckadoo 1 & 910 & 60 & Walkunder Arch Cave \\
\hline 1160 & 140 & Echidna Shelter & 900 & 50 & Jiyer Cave \\
\hline 1150 & 90 & Chillagoe Site 55 Rock Art & 900 & 80 & Kwamter Mound \\
\hline 1150 & 60 & Echidna's Rest & 900 & 50 & Walkunder Arch Cave \\
\hline 1150 & 70 & First Ridge & 890 & 60 & Bayley Point Mound 3 \\
\hline 1150 & 80 & Lake Numalla II & 890 & 70 & Kwamter Mound \\
\hline 1150 & 50 & Quinine Bush Shelter & 890 & 50 & Kyeenee I \\
\hline 1140 & 110 & Fern Cave & 890 & 50 & Ngarrabullgan Cave \\
\hline 1140 & 100 & Mitchell River Cave & 890 & 50 & Ngarrabullgan Cave \\
\hline 1130 & 130 & Mt Roundback & 890 & 80 & Weipa 25 \\
\hline 1130 & 60 & Stockyard Point & 890 & 70 & Weipa Mound M509 \\
\hline 1130 & 60 & Wreck Beach & 880 & 60 & Curlew Island Open Site \\
\hline 1120 & 60 & New Brisbane Airport & 880 & 50 & Gyranda 13 \\
\hline 1120 & 430 & Red Island Point & 880 & 70 & Tom's Creek Site Complex \\
\hline 1120 & 70 & Weipa Mound M39 & 880 & 70 & Whites Bore IV \\
\hline 1110 & 91 & Broadbeach Burial Ground & 870 & 60 & Ngarrabullgan Cave \\
\hline 1110 & 10 & South Mound & 870 & 130 & Pancake Creek Site Complex \\
\hline 1110 & 70 & Tom's Creek Site Complex & 870 & 70 & Weipa 16 \\
\hline 1100 & 70 & Mushroom Rock East & 870 & 80 & Weipa Mound M3 \\
\hline 1090 & 70 & Gatton Shelter & 860 & 80 & Wunjunga BMO \\
\hline 1090 & 70 & Lake Numalla I & 855 & 80 & Kwamter Mound \\
\hline 1080 & 50 & Ngarrabullgan Cave & 850 & 125 & Herveys Range B \\
\hline 1080 & NA & Quinkan B6 Rockshelter Rock Art & 850 & 100 & Midden in Ridge 4 \\
\hline 1070 & 70 & Teewah Beach 26 & 840 & 70 & Echidna's Rest Rock Art \\
\hline
\end{tabular}


SECTION 8: Index of Sites by Conventional Radiocarbon Age

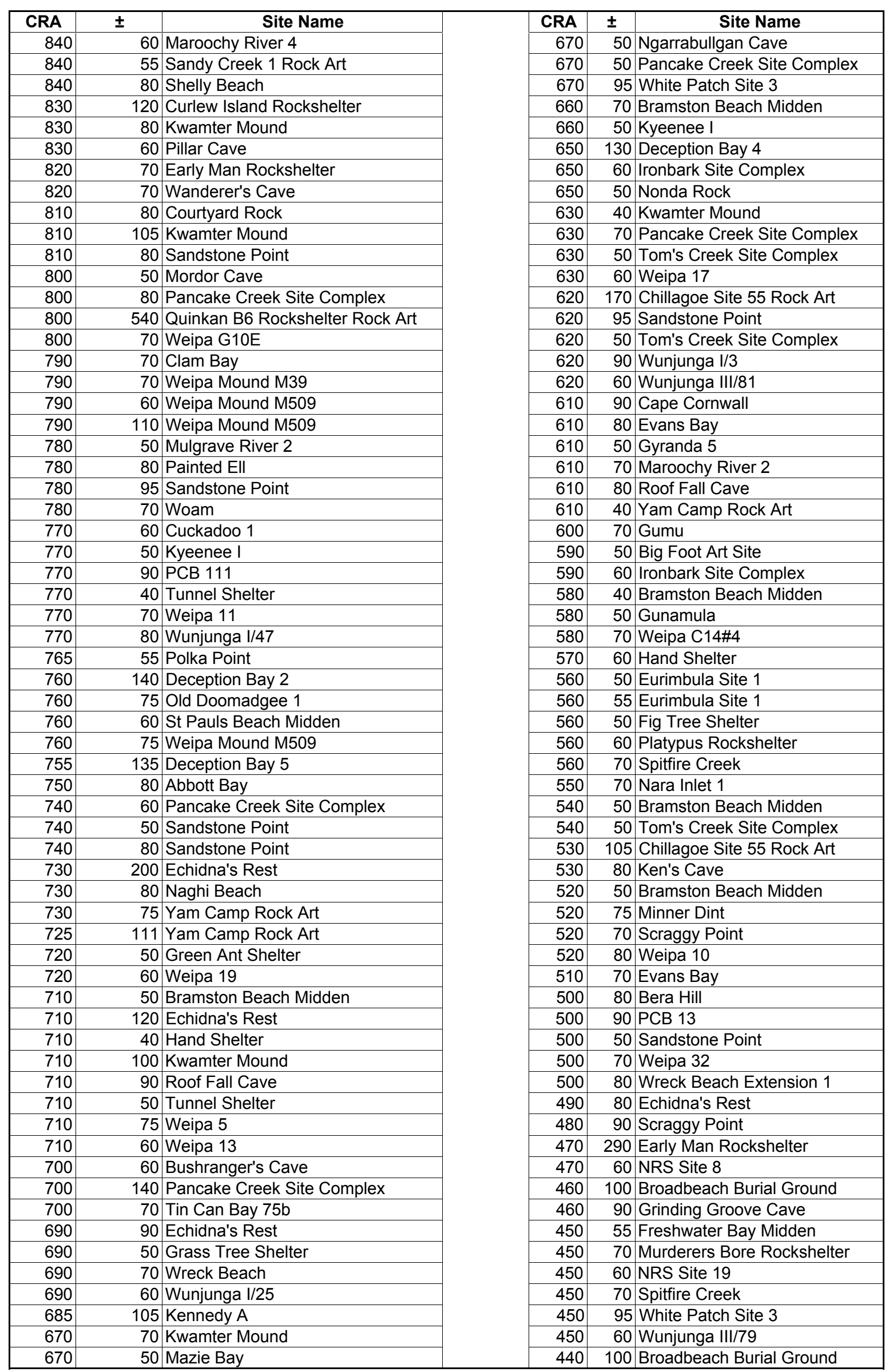


SECTION 8: Index of Sites by Conventional Radiocarbon Age

\begin{tabular}{|c|c|c|c|c|c|}
\hline CRA & \pm & Site Name & CRA & \pm & Site Name \\
\hline 440 & 55 & Echidna's Rest Rock Art & 240 & 60 & Mulgrave River 1 \\
\hline 440 & 60 & Eurimbula Site 1 & 235 & 110 & Kwamter Mound \\
\hline 430 & 70 & Kaponyee Springs II & 220 & 80 & Eurimbula Site 1 \\
\hline 430 & 60 & NE Moreton Island 1 & 220 & 70 & Monkey Point \\
\hline 430 & 70 & Sandstone Point & 220 & 50 & Weipa 33 \\
\hline 420 & 100 & PCB 13 & 200 & 140 & Ironbark Site Complex \\
\hline 410 & 50 & NRS Site 22 & 200 & 80 & Jiyer Cave \\
\hline 400 & 60 & Echidna's Rest Rock Art & 190 & 50 & Cameron Point 62 \\
\hline 400 & 110 & Kaponyee Springs II & 180 & 50 & Weipa 9 \\
\hline 400 & 60 & NE Moreton Island 16 & 170 & 60 & Maroochy River 4 \\
\hline 380 & 80 & Saibai Village Store & 140 & 60 & Mulgrave River 2 \\
\hline 370 & 75 & Toulkerrie & 100 & 60 & Jiyer Cave \\
\hline 360 & 100 & Weipa Mound M509 & 70 & 70 & Kabali Road Scatter \\
\hline 350 & 80 & Gainsford 2 & 50 & 86 & Broadbeach Burial Ground \\
\hline 350 & 55 & Hay Cave & 0 & 0 & Cape Flattery \\
\hline 350 & 70 & Toulkerrie & 0 & 0 & Echidna's Rest Rock Art \\
\hline 350 & 40 & Wunjunga I/3 & 0 & 0 & Echidna's Rest Rock Art \\
\hline 340 & 70 & Sandstone Point & 0 & 0 & Echidna's Rest Rock Art \\
\hline 340 & 70 & Teewah Beach 26 & 0 & 0 & Echidna's Rest Rock Art \\
\hline 300 & 60 & Gyranda 5 & 0 & 0 & Sandstone Point \\
\hline 280 & 60 & Spur Bay East & 0 & 0 & Seary-Broutha Site \\
\hline 270 & 70 & Weipa 30 & 0 & 0 & Teewah Beach 5D \\
\hline 260 & 50 & Jiyer Cave & 0 & 0 & Tom's Creek Site Complex \\
\hline 250 & 70 & Big Sandhills Beach & 0 & 0 & Webber Swamp 100 \\
\hline 250 & 50 & Saint-Smith Midden & 0 & 0 & Youlain Springs \\
\hline
\end{tabular}




\section{SECTION 9:}

\section{Index of Sites by Region}




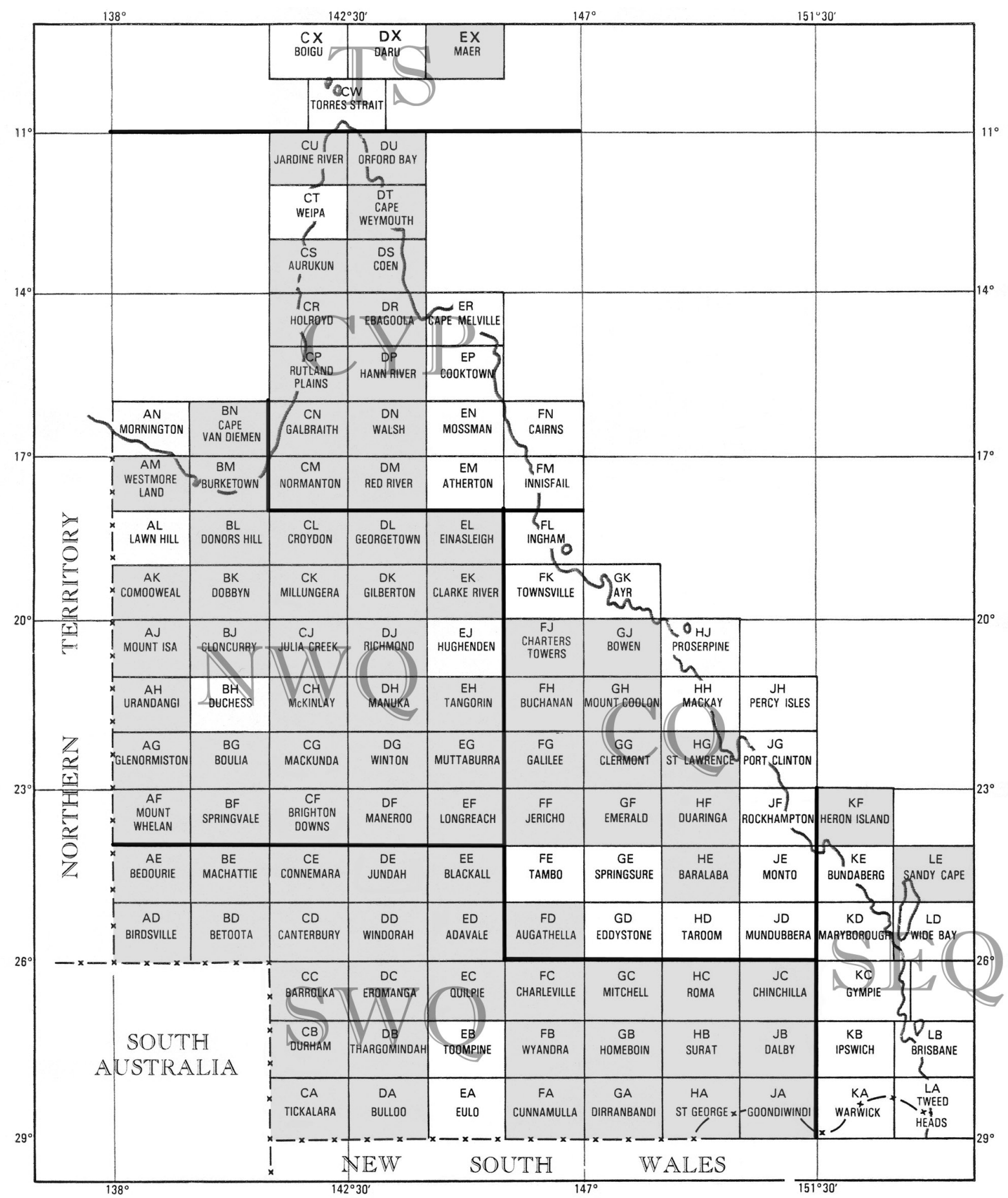

Figure 4. 1:250,000 map sheets with superimposed regions used to structure geographical information in the Index (after Johnston and Rowland 1987). Shaded squares indicate that no dated archaeological sites occur on that map sheet. The two letter code above each map sheet name is used by the Queensland Environmental Protection Agency to code cultural heritage places occurring on that map sheet. 
SECTION 9: Index of Sites by Region

\begin{tabular}{|c|c|c|c|}
\hline Region & Site Name & Region & Site Name \\
\hline \multirow[t]{58}{*}{$\mathrm{CQ}$} & Abbott Bay & \multirow[t]{63}{*}{ CYP } & Chillagoe Site 62 Rock Art \\
\hline & Big Foot Art Site & & Chillagoe Site 75 Rock Art \\
\hline & Big Sandhills Beach & & Courtyard Rock \\
\hline & Border Island 1 & & Deighton Lady Rock Art \\
\hline & Boulder 1 & & Dragonfly Hollow \\
\hline & Cape Palmerston & & Early Man Rockshelter \\
\hline & Castle Rock Cave & & Echidna's Rest \\
\hline & Cathedral Cave & & Echidna Shelter \\
\hline & Clam Bay & & Endaen Rockshelter \\
\hline & Curlew Island Open Site & & Evans Bay \\
\hline & Curlew Island Rockshelter & & Fern Cave \\
\hline & Gainsford 1 & & Fig Tree Shelter \\
\hline & Gainsford 2 & & Freshwater Bay Midden \\
\hline & Goat Rock & & Giant Horse \\
\hline & Grinding Groove Cave & & Gorge Creek Shelter \\
\hline & Gyranda 5 & & Grass Tree Shelter \\
\hline & Gyranda 8 & & Green Ant Shelter \\
\hline & Gyranda 13 & & Hand Shelter \\
\hline & Gyranda 17 & & Hay Cave \\
\hline & Herveys Range B & & Hearth Cave \\
\hline & Hill Inlet 1 & & Initiation Cave \\
\hline & Jourama Rockshelter & & Jiyer Cave \\
\hline & Kennedy A & & Kennedy River (Shelter B) Rock Art \\
\hline & Kenniff Cave & & Kookaburra Rock \\
\hline & Ken's Cave & & Kwamter Mound \\
\hline & Leefe Peak & & Lookout Shelter \\
\hline & Marble Island & & Magnificent Gallery \\
\hline & Mazie Bay & & Midden in Ridge 4 \\
\hline & Monkey Beach & & Midden in Ridge 5 \\
\hline & Monkey Point & & Mitchell River Cave \\
\hline & Mt Roundback & & Mordor Cave \\
\hline & Nara Inlet 1 & & Mother Mound on Ridge 3 \\
\hline & Nara Inlet Art Site & & Mulgrave River 1 \\
\hline & Native Well 1 & & Mulgrave River 2 \\
\hline & Native Well 2 & & Mungana Site Rock Art \\
\hline & Rainbow Cave & & Mushroom Rock West \\
\hline & Rainbow Cave 2 & & Mushroom Rock East \\
\hline & Red Beach & & Ngarrabullgan Cave \\
\hline & Road Cave 1 & & Nonda Rock \\
\hline & Roof Fall Cave & & Painted Ell \\
\hline & Scraggy Point & & PCB 13 \\
\hline & Shelly Beach & & PCB 111 \\
\hline & Spur Bay East & & PCB 141 \\
\hline & St Bees Island Rockshelter & & Pillar Cave \\
\hline & Stockyard Point & & Platform Gallery Rockshelter \\
\hline & The Tombs Rockshelter & & Quinine Bush Shelter \\
\hline & Townsville Common & & Quinkan B6 Rockshelter Rock Art \\
\hline & Turtle Rock & & Racecourse Site Rock Art \\
\hline & Turtle Rock Rockshelter & & Red Bluff 1 \\
\hline & Wanderer's Cave & & Red Horse \\
\hline & Wreck Beach & & Red Island Point \\
\hline & Wreck Beach Extension 1 & & Red Lady Rock Art \\
\hline & Wunjunga BMO & & Sandy Creek 1 \\
\hline & Wunjunga I/3 & & Sandy Creek 2 \\
\hline & Wunjunga $\mathrm{I} / 25$ & & South Mound \\
\hline & Wunjunga I/47 & & Split Rock Rock Art \\
\hline & Wunjunga III/79 & & Tunnel Shelter \\
\hline & Wunjunga III/81 & & Walaemini Rockshelter \\
\hline \multirow[t]{5}{*}{ CYP } & Alkaline Hill & & Walkunder Arch Cave \\
\hline & Bramston Beach Midden & & Weipa 5 \\
\hline & Bush Peg Shelter & & Weipa 6 \\
\hline & Cape Flattery & & Weipa 7 \\
\hline & Chillagoe Site 55 Rock Art & & Weipa 8 \\
\hline
\end{tabular}


SECTION 9: Index of Sites by Region

\begin{tabular}{|c|c|c|c|}
\hline Region & Site Name & Region & Site Name \\
\hline \multirow{33}{*}{ CYP } & Weipa 9 & \multirow[t]{51}{*}{ SEQ } & Deception Bay 5 \\
\hline & Weipa 10 & & Double Island Point 1 \\
\hline & Weipa 11 & & Eurimbula Creek 1 \\
\hline & Weipa 12 & & Eurimbula Creek 2 \\
\hline & Weipa 13 & & Eurimbula Site 1 \\
\hline & Weipa 14 & & First Ridge \\
\hline & Weipa 16 & & Fraser Island 217/15 \\
\hline & Weipa 17 & & Fraser Island 796/54 \\
\hline & Weipa 18 & & Fraser Island 799/54 \\
\hline & Weipa 19 & & Gatton Shelter \\
\hline & Weipa 20 & & Hope Island \\
\hline & Weipa 20A & & Ironbark Site Complex \\
\hline & Weipa 21 & & Kabali Road Scatter \\
\hline & Weipa 23 & & King's Bore Site 97 \\
\hline & Weipa 24 & & Lake Coombabah \\
\hline & Weipa 25 & & Little Sandhills \\
\hline & Weipa 26 & & Maidenwell Shelter \\
\hline & Weipa 27 & & Maroochy River 2 \\
\hline & Weipa 28 & & Maroochy River 4 \\
\hline & Weipa 29 & & Minner Dint \\
\hline & Weipa 30 & & Middle Island Sandblow Site \\
\hline & Weipa 31 & & Moon Point \\
\hline & Weipa 32 & & Mort Creek Site Complex \\
\hline & Weipa 33 & & New Brisbane Airport \\
\hline & Weipa C14\#4 & & NE Moreton Island 1 \\
\hline & Weipa G10E & & NE Moreton Island 5 \\
\hline & Weipa Mound M3 & & NE Moreton Island 16 \\
\hline & Weipa Mound M34 & & NE Moreton Island 21A \\
\hline & Weipa Mound M37 & & NE Moreton Island 27 \\
\hline & Weipa Mound M39 & & NRS Site 8 \\
\hline & Weipa Mound M39b & & NRS Site 19 \\
\hline & Weipa Mound M509 & & NRS Site 22 \\
\hline & Yam Camp & & One-Tree \\
\hline \multirow[t]{13}{*}{ NWQ } & Bayley Point Mound 3 & & Pancake Creek Site Complex \\
\hline & Colless Creek Cave & & Platypus Rockshelter \\
\hline & Cuckadoo 1 & & Polka Point \\
\hline & DH Site & & Saint-Smith Midden \\
\hline & Gunamula & & Sandstone Point \\
\hline & Louie Creek Cave & & Seary-Broutha Site \\
\hline & Mickey Springs 31 & & Seven Mile Creek Mound \\
\hline & Mickey Springs 33 & & Spitfire Creek \\
\hline & Mickey Springs 34 & & St Helena Island \\
\hline & Old Doomadgee 1 & & Talgai Skull \\
\hline & Quippenburra Cave & & Teewah Beach 5D \\
\hline & Selwyn Ranges 1 & & Teewah Beach 26 \\
\hline & Selwyn Ranges 2 & & Tin Can Bay 75b \\
\hline \multirow[t]{17}{*}{ SEQ } & Aranarawai Beach Ridge II & & Tom's Creek Site Complex \\
\hline & Bishop's Peak & & Toulkerrie \\
\hline & Boonah Shelter & & Wallen Wallen Creek \\
\hline & Booral Homestead Midden & & Webber Swamp 100 \\
\hline & Booral Shell Mound & & White Patch Site 3 \\
\hline & Bribie Island 9 & \multirow[t]{10}{*}{ SWQ } & Kaponyee Springs I \\
\hline & Broadbeach Burial Ground & & Kaponyee Springs II \\
\hline & Brooyar Rockshelter & & Kyeenee I \\
\hline & Brown's Road & & Lake Numalla I \\
\hline & Bushranger's Cave & & Lake Numalla II \\
\hline & Cameron Point 62 & & Lake Toomaroo \\
\hline & Christmas Creek & & Murderers Bore Rockshelter \\
\hline & Cribb Island & & Whites Bore III \\
\hline & Deception Bay 1 & & Whites Bore IV \\
\hline & Deception Bay 2 & & Youlain Springs \\
\hline & Deception Bay 3 & \multirow[t]{2}{*}{ TS } & Bera Hill \\
\hline & Deception Bay 4 & & Cape Cornwall \\
\hline
\end{tabular}


SECTION 9: Index of Sites by Region

\begin{tabular}{|c|c|c|c|}
\hline Region & Site Name & Region & Site Name \\
\hline \multirow{3}{*}{ TS } & Gumu & \multirow{3}{*}{ TS } & Saibai Village Store \\
\hline & Long Beach & & St Pauls Beach Midden \\
\hline & Naghi Beach & & Woam \\
\hline
\end{tabular}




\section{SECTION 10:}

\section{Index of Sites by 1:250,000 Map Sheet Name}




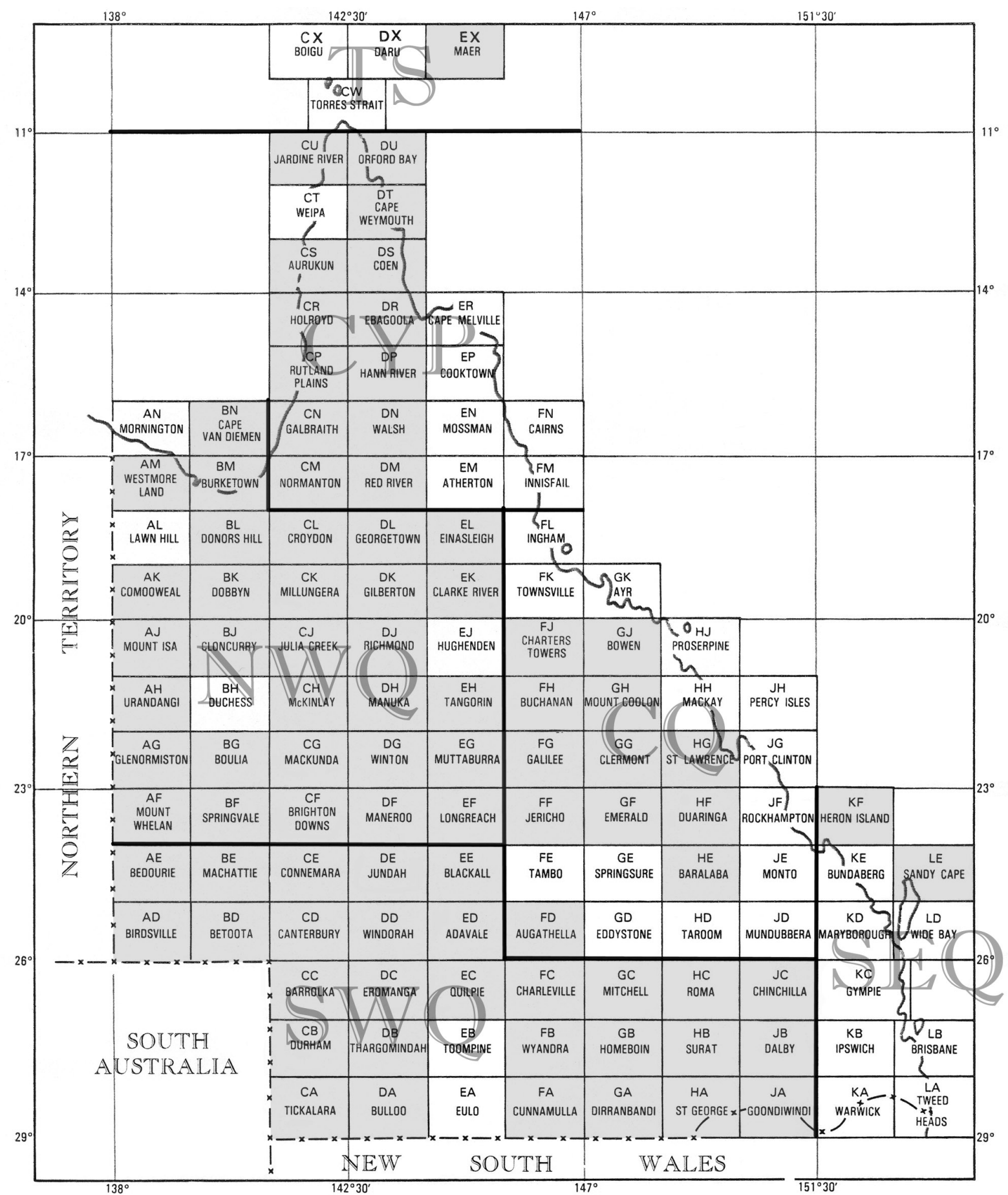

Figure 5. 1:250K map sheets with superimposed regions used to structure geographical information in the Index (after Johnston and Rowland 1987). Shaded squares indicate that no archaeological sites occur on that map sheet. The two letter code above each map sheet name is used by the Queensland Environmental Protection Agency to code cultural heritage places occurring on that map sheet. 
SECTION 10: Index of Sites by 1:250,000 Map Sheet Name

\begin{tabular}{|c|c|c|c|}
\hline 1:250K Sheet & Site Name & 1:250K Sheet & Site Name \\
\hline \multirow[t]{10}{*}{ Atherton } & Chillagoe Site 55 Rock Art & \multirow[t]{11}{*}{ Cape Melville } & Alkaline Hill \\
\hline & Chillagoe Site 62 Rock Art & & Endaen Rockshelter \\
\hline & Chillagoe Site 75 Rock Art & & Freshwater Bay Midden \\
\hline & Echidna's Rest & & Midden in Ridge 4 \\
\hline & Echidna Shelter & & Midden in Ridge 5 \\
\hline & Fern Cave & & Mother Mound on Ridge 3 \\
\hline & Mungana Site Rock Art & & PCB 13 \\
\hline & Pillar Cave & & PCB 111 \\
\hline & Racecourse Site Rock Art & & PCB 141 \\
\hline & Walkunder Arch Cave & & South Mound \\
\hline \multirow[t]{10}{*}{ Ayr } & Abbott Bay & & Walaemini Rockshelter \\
\hline & Gainsford 1 & \multirow[t]{17}{*}{ Cooktown } & Cape Flattery \\
\hline & Gainsford 2 & & Deighton Lady Rock Art \\
\hline & Mt Roundback & & Early Man Rockshelter \\
\hline & Wunjunga BM0 & & Giant Horse \\
\hline & Wunjunga $\mathrm{I} / 3$ & & Green Ant Shelter \\
\hline & Wunjunga I/25 & & Kennedy River (Shelter B) Rock Art \\
\hline & Wunjunga I/47 & & Magnificent Gallery \\
\hline & Wunjunga III/79 & & Mushroom Rock East \\
\hline & Wunjunga III/81 & & Mushroom Rock West \\
\hline Boigu & Gumu & & Platform Gallery Rockshelter \\
\hline \multirow[t]{31}{*}{ Brisbane } & Aranarawai Beach Ridge II & & Quinkan B6 Rockshelter Rock Art \\
\hline & Brown's Road & & Red Bluff 1 \\
\hline & Cribb Island & & Red Horse \\
\hline & Deception Bay 1 & & Sandy Creek 1 \\
\hline & Deception Bay 2 & & Sandy Creek 2 \\
\hline & Deception Bay 3 & & Split Rock Rock Art \\
\hline & Deception Bay 4 & & Yam Camp \\
\hline & Deception Bay 5 & \multirow[t]{2}{*}{ Daru } & Saibai Village Store \\
\hline & First Ridge & & Woam \\
\hline & Hope Island & \multirow[t]{3}{*}{ Duchess } & Cuckadoo 1 \\
\hline & Lake Coombabah & & Selwyn Ranges 1 \\
\hline & Little Sandhills & & Selwyn Ranges 2 \\
\hline & Minner Dint & \multirow[t]{5}{*}{ Eddystone } & Cathedral Cave \\
\hline & New Brisbane Airport & & Goat Rock \\
\hline & NE Moreton Island 1 & & Native Well 1 \\
\hline & NE Moreton Island 5 & & Native Well 2 \\
\hline & NE Moreton Island 16 & & The Tombs Rockshelter \\
\hline & NE Moreton Island 21A & \multirow[t]{9}{*}{ Eulo } & Kaponyee Springs I \\
\hline & NE Moreton Island 27 & & Kaponyee Springs II \\
\hline & NRS Site 8 & & Lake Numalla I \\
\hline & NRS Site 19 & & Lake Numalla II \\
\hline & NRS Site 22 & & Lake Toomaroo \\
\hline & One-Tree & & Murderers Bore Rockshelter \\
\hline & Polka Point & & Whites Bore III \\
\hline & Saint-Smith Midden & & Whites Bore IV \\
\hline & Sandstone Point & & Youlain Springs \\
\hline & Spitfire Creek & \multirow[t]{9}{*}{ Gympie } & Bribie Island 9 \\
\hline & St Helena Island & & Brooyar Rockshelter \\
\hline & Toulkerrie & & King's Bore Site 97 \\
\hline & Wallen Wallen Creek & & Maidenwell Shelter \\
\hline & White Patch Site 3 & & Maroochy River 2 \\
\hline \multirow[t]{9}{*}{ Bundaberg } & Eurimbula Creek 1 & & Maroochy River 4 \\
\hline & Eurimbula Creek 2 & & Seary-Broutha Site \\
\hline & Eurimbula Site 1 & & Teewah Beach 5D \\
\hline & Ironbark Site Complex & & Teewah Beach 26 \\
\hline & Middle Island Sandblow Site & \multirow[t]{4}{*}{ Hughenden } & Mickey Springs 31 \\
\hline & Mort Creek Site Complex & & Mickey Springs 33 \\
\hline & Pancake Creek Site Complex & & Mickey Springs 34 \\
\hline & Seven Mile Creek Mound & & Quippenburra Cave \\
\hline & Tom's Creek Site Complex & \multirow[t]{3}{*}{ Ingham } & Jourama Rockshelter \\
\hline \multirow[t]{2}{*}{ Cairns } & Mulgrave River 1 & & Kennedy A \\
\hline & Mulgrave River 2 & & Leefe Peak \\
\hline
\end{tabular}


SECTION 10: Index of Sites by 1:250,000 Map Sheet Name

\begin{tabular}{|c|c|c|c|}
\hline 1:250K Sheet & Site Name & 1:250K Sheet & Site Name \\
\hline Ingham & Scraggy Point & Springsure & Turtle Rock \\
\hline \multirow[t]{2}{*}{ Innisfail } & Bramston Beach Midden & \multirow[t]{2}{*}{ Tambo } & Ken's Cave \\
\hline & Jiyer Cave & & Rainbow Cave 2 \\
\hline \multirow[t]{2}{*}{ Ipswich } & Gatton Shelter & \multirow[t]{2}{*}{ Taroom } & Rainbow Cave \\
\hline & Platypus Rockshelter & & Wanderer's Cave \\
\hline \multirow[t]{3}{*}{ Lawn Hill } & Colless Creek Cave & Toompine & Kyeenee I \\
\hline & DH Site & \multirow[t]{7}{*}{ Torres Strait } & Bera Hill \\
\hline & Louie Creek Cave & & Cape Cornwall \\
\hline \multirow[t]{3}{*}{ Mackay } & Cape Palmerston & & Evans Bay \\
\hline & Curlew Island Open Site & & Long Beach \\
\hline & Curlew Island Rockshelter & & Naghi Beach \\
\hline \multirow[t]{3}{*}{ Maryborough } & Booral Homestead Midden & & Red Island Point \\
\hline & Booral Shell Mound & & St Pauls Beach Midden \\
\hline & Moon Point & \multirow[t]{3}{*}{ Townsville } & Herveys Range B \\
\hline \multirow[t]{5}{*}{ Monto } & Big Foot Art Site & & Townsville Common \\
\hline & Boulder 1 & & Turtle Rock Rockshelter \\
\hline & Grinding Groove Cave & \multirow[t]{4}{*}{ Tweed Heads } & Bishop's Peak \\
\hline & Road Cave 1 & & Broadbeach Burial Ground \\
\hline & Roof Fall Cave & & \begin{tabular}{|l|} 
Bushranger's Cave \\
\end{tabular} \\
\hline \multirow[t]{3}{*}{ Mornington } & Bayley Point Mound 3 & & Christmas Creek \\
\hline & Gunamula & \multirow[t]{2}{*}{ Warwick } & Boonah Shelter \\
\hline & Old Doomadgee 1 & & Talgai Skull \\
\hline \multirow[t]{19}{*}{ Mossman } & Bush Peg Shelter & \multirow[t]{37}{*}{ Weipa } & Kwamter Mound \\
\hline & Courtyard Rock & & Weipa 5 \\
\hline & Dragonfly Hollow & & Weipa 6 \\
\hline & Fig Tree Shelter & & Weipa 7 \\
\hline & Gorge Creek Shelter & & Weipa 8 \\
\hline & Grass Tree Shelter & & Weipa 9 \\
\hline & Hand Shelter & & Weipa 10 \\
\hline & Hay Cave & & Weipa 11 \\
\hline & Hearth Cave & & Weipa 12 \\
\hline & Initiation Cave & & Weipa 13 \\
\hline & Kookaburra Rock & & Weipa 14 \\
\hline & Lookout Shelter & & Weipa 16 \\
\hline & Mitchell River Cave & & Weipa 17 \\
\hline & Mordor Cave & & Weipa 18 \\
\hline & Ngarrabullgan Cave & & Weipa 19 \\
\hline & Nonda Rock & & Weipa 20 \\
\hline & Painted Ell & & Weipa 20A \\
\hline & Quinine Bush Shelter & & Weipa 21 \\
\hline & Tunnel Shelter & & Weipa 23 \\
\hline Mundubbera & Gyranda 5 & & Weipa 24 \\
\hline & Gyranda 8 & & Weipa 25 \\
\hline & Gyranda 13 & & Weipa 26 \\
\hline & Gyranda 17 & & Weipa 27 \\
\hline Percy Isles & Castle Rock Cave & & Weipa 28 \\
\hline & Marble Island & & Weipa 29 \\
\hline & Spur Bay East & & Weipa 30 \\
\hline Port Clinton & Stockyard Point & & Weipa 31 \\
\hline Proserpine & Border Island 1 & & Weipa 32 \\
\hline & Hill Inlet 1 & & Weipa 33 \\
\hline & Nara Inlet 1 & & Weipa C14\#4 \\
\hline & Nara Inlet Art Site & & Weipa G10E \\
\hline & St Bees Island Rockshelter & & Weipa Mound M3 \\
\hline Rockhampton & Big Sandhills Beach & & Weipa Mound M34 \\
\hline & Clam Bay & & Weipa Mound M37 \\
\hline & Mazie Bay & & Weipa Mound M39 \\
\hline & Monkey Beach & & Weipa Mound M39b \\
\hline & Monkey Point & & Weipa Mound M509 \\
\hline & Red Beach & Wide Bay & Cameron Point 62 \\
\hline & Wreck Beach & & Double Island Point 1 \\
\hline & Wreck Beach Extension 1 & & Fraser Island 217/15 \\
\hline Springsure & Kenniff Cave & & Fraser Island 796/54 \\
\hline
\end{tabular}


SECTION 10: Index of Sites by 1:250,000 Map Sheet Name

\begin{tabular}{|c|c|c|c|}
\hline 1:250K Sheet & Site Name & 1:250K Sheet & Site Name \\
\hline \multirow[t]{4}{*}{ Wide Bay } & Fraser Island 799/54 & \multirow[t]{2}{*}{ Unknown } & Red Lady Rock Art \\
\hline & Kabali Road Scatter & & Shelly Beach \\
\hline & Tin Can Bay 75b & & \\
\hline & Webber Swamp 100 & & \\
\hline
\end{tabular}




\section{SECTION 11:}

\section{References Cited}


ABC 1979 Exercise Logan Jack. Captain Les Hiddens. Canberra: Australian Broadcasting Commission.

Abbie, A.A. 1951 The Australian Aborigine. Oceania 22(2):91-100.

Aitchison, T.C., M. Leese, D.J. Michczynska, W.G. Mook, R.L. Otlet, B.S. Ottaway, M.F. Pazdur, J. van der Plicht, P.J. Reimer, S.W. Robinson, E.M. Scott, M. Stuiver and B. Weninger 1989 A comparison of methods used for the calibration of radiocarbon dates. Radiocarbon 31(3):846-862.

Aitken, M.J. 1990 Science-Based Dating in Archaeology. London: Longman.

Alfredson, G. 1983 St Helena Island - A changing pattern of exploitation? Australian Archaeology 17:79-86.

Alfredson, G. 1984a An Archaeological Investigation into the Aboriginal Use of St Helena Island, Moreton Bay. Unpublished B.A. (Hons) thesis, Department of Anthropology and Sociology, University of Queensland, Brisbane.

Alfredson, G. 1984b The Aboriginal use of St Helena Island, Moreton Bay - The archaeological evidence. In R.J. Coleman, J. Covacevich and P. Davie (eds), Focus on Stradbroke: New Information on North Stradbroke Island and Surrounding Areas, 1974-1984, pp.1-8. Brisbane: Boolarong Publications.

Alfredson, G. and Kombumerri Aboriginal Corporation for Culture 1998 A Report on an Initial Cultural Heritage Assessment of a Proposed Golf Course on Lake Coombabah. Unpublished report to the Nimmel Partnership, Mudgeeraba, Queensland.

Alfredson, G. and Kombumerri Aboriginal Corporation for Culture 1999 Report on a Collection and a Further Cultural Heritage Assessment for a Proposed Golf Course and Residential Development on Lake Coombabah. Unpublished report to the Nimmel Partnership, Mudgeeraba, Queensland.

Anon. 1967 Recent Australian radiocarbon dates. Australian Institute of Aboriginal Studies Newsletter 2(6):20-35.

Aplin, K. n.d. Report on Analysis of the Colless Creek Rockshelter Archaeological Fauna. Unpublished report to the Australian Heritage Commission, Canberra.

Archer, M. 1977 Recent Local Faunas from Excavations at Hervey's Range, Kennedy Cave, Jourama and Mt Roundback, North-Eastern Queensland; Summary of Human Bone, with Comments. In H.C. Brayshaw, Aboriginal Material Culture in the Herbert/Burdekin District, North Queensland, pp.655-689. Unpublished PhD thesis, James Cook University of North Queensland, Townsville.

Archer, M. and H.C. Brayshaw 1978 Recent local faunas from excavations at Hervey's Range, Kennedy, Jourama, and Mount Roundback, north-eastern Queensland. Memoirs of the Queensland Museum 18(2):165-177.

Armitage, R.A., B. David, M. Hyman, M.W. Rowe, C. Tuniz, E. Lawson, G. Jacobsen and Q. Hua 1998
Radiocarbon determinations on Chillagoe rock paintings: Small sample accelerator mass spectrometry. Records of the Australian Museum 50(3):285-292.

Armitage, R.A., M. Hyman and M.W. Rowe 2000 Plasmachemistry for dating pictographs by AMS. In G.K. Ward and C. Tuniz (eds), Advances in Dating Australian Rock-Markings: Papers from the First Australian Rock-Picture Dating Workshop, pp.31-34. Occasional AURA Publication 10. Melbourne: Australian Rock Art Research Association.

Bailey, G.N. 1975 The Role of Shell Middens in Prehistoric Economics. Unpublished $\mathrm{PhD}$ thesis, Cambridge University, Cambridge.

Bailey, G.N. 1977 Shell mounds, shell middens, and raised beaches in the Cape York Peninsula. Mankind 11(2):132-143.

Bailey, G. 1994 The Weipa shell mounds: Natural or cultural? In M. Sullivan, S. Brockwell and A. Webb (eds), Archaeology in the North: Proceedings of the 1993 Australian Archaeological Association Conference, pp.107-129. Darwin: North Australia Research Unit, Australian National University.

Bailey, G., J. Chappell and R. Cribb 1994 The origin of Anadara shell mounds at Weipa, north Queensland, Australia. Archaeology in Oceania 29(2):69-80.

Barham, A.J. 1981 Land Use and Environmental Change in the Western Torres Strait Islands, Northern Queensland, Australia. Fieldwork Report. Unpublished report to Department of Geography, University College London and Institute of Archaeology, University of London.

Barham, A.J. 1999 The local environmental impact of prehistoric populations on Saibai Island, northern Torres Strait, Australia: Enigmatic evidence from Holocene swamp lithostratigraphic records. Quaternary International 59:71-105.

Barham, A.J. 2000 Late Holocene maritime societies in the Torres Strait Islands, northern Australia - Cultural arrival or cultural emergence? In S. O'Connor and P. Veth (eds), East of Wallace's Line: Studies of Past and Present Maritime Cultures of the Indo-Pacific Region, pp.223-314. Modern Quaternary Research in Southeast Asia 16. Rotterdam: Balkema.

Barham, A.J. and D.R. Harris 1983 Prehistory and palaeoecology of Torres Strait. In P.M. Masters and N.C. Fleming (eds), Quaternary Coastlines and Marine Archaeology: Towards the Prehistory of Land Bridges and Continental Shelves, pp.529-557. London: Academic Press.

Barham, A.J. and D.R. Harris 1985 Relict field systems in the Torres Strait region. In I.S. Farrington (ed.), Prehistoric Intensive Agriculture in the Tropics, pp.247-283. BAR International Series 232. Oxford: British Archaeological Reports.

Barham, A.J., M.J. Rowland and G.J. Hitchcock in prep. Torres Strait bepotaim: An overview of archaeological and ethnoarchaeological investigations and research. To be submitted to Memoirs of the Queensland Museum, 


\section{Cultural Heritage Series.}

Barker, B.C. 1989 Nara Inlet 1: A Holocene sequence from the Whitsunday Islands, central Queensland coast. Queensland Archaeological Research 6:53-76.

Barker, B.C. 1991 Nara Inlet 1: Coastal resource use and the Holocene marine transgression in the Whitsunday Islands, central Queensland. Archaeology in Oceania 26(3):102-109.

Barker, B. 1993 Early human exploitation of island environments within the Great Barrier Reef Marine Park. Reef Research 3(3):13-14.

Barker, B. 1995 'The Sea People': Maritime HunterGatherers on the Tropical Coast: A Late Holocene Maritime Specialisation in the Whitsunday Islands, Central Queensland. Unpublished $\mathrm{PhD}$ thesis, Department of Anthropology and Sociology, University of Queensland, Brisbane.

Barker, B. 1996 Maritime hunter-gatherers on the tropical coast: A social model for change. In S. Ulm, I. Lilley and A. Ross (eds), Australian Archaeology '95: Proceedings of the 1995 Australian Archaeological Association Annual Conference, pp.31-43. Tempus 6. St Lucia: Anthropology Museum, Department of Anthropology and Sociology, University of Queensland.

Barker, B. 1999 Coastal occupation in the late Holocene: Environment, resource use and resource continuity. In J. Hall and I.J. McNiven (eds), Australian Coastal Archaeology, pp.119-127. Research Papers in Archaeology and Natural History 31. Canberra: ANH Publications, Department of Archaeology and Natural History, Research School of Pacific and Asian Studies, Australian National University.

Barker, B., K. Sale and A. Watchman 1997 Authentication of rock art in the Whitsunday Islands, Queensland, Australia. Rock Art Research 14(2):113-123.

Barker, B. and R. Schon 1994 A preliminary assessment of the spatial distribution of stone artefacts from the South Molle Island Aboriginal quarry, Whitsunday Islands, central Queensland coast. Memoirs of the Queensland Museum 37(1):5-12.

Bartholomai, A. 1965 The Faunal Material Excavated from the Cathedral Cave. Unpublished paper, Queensland Museum, Brisbane.

Bartholomai, A. 1976 Faunal remains excavated from site Q1, Broadbeach, southeast Queensland. Appendix B. In L. Haglund, The Broadbeach Aboriginal Burial Ground: An Archaeological Analysis, pp.90-93. St Lucia: University of Queensland Press.

Beaton, J.M. 1977 Dangerous Harvest: Investigations in the Late Prehistoric Occupation of Upland South-East Central Queensland. Unpublished $\mathrm{PhD}$ thesis, Department of Prehistory, Research School of Pacific and Asian Studies, Australian National University, Canberra.

Beaton, J. 1981 Princess Charlotte Bay Archaeological Report: Second Interim Report. Unpublished report to the Australian Institute of Aboriginal and Torres Strait
Islander Studies, Canberra.

Beaton, J. 1985 Evidence for a coastal occupation time-lag at Princess Charlotte Bay (North Queensland) and implications for coastal colonisation and population growth theories for Aboriginal Australia. Archaeology in Oceania 20(1):1-20.

Beaton, J.M. 1991a Excavations at Rainbow Cave and Wanderer's Cave: Two rockshelters in the Carnarvon Range, Queensland. Queensland Archaeological Research 8:2-32.

Beaton, J.M. 1991b Cathedral Cave: A rockshelter in Carnarvon Gorge, Queensland. Queensland Archaeological Research 8:33-84.

Belperio, A.P. 1978 An Inner Shelf Sedimentation Model for the Townsville Region, Great Barrier Reef Province. Unpublished $\mathrm{PhD}$ thesis, James Cook University of North Queensland, Townsville.

Bermingham, A. 1966 Victoria natural radiocarbon measurements I. Radiocarbon 8:507-521.

Bermingham, A. 1971 Australian natural radiocarbon measurements. Australian Institute of Aboriginal Studies Newsletter 3(2):30-35.

Bird, M.I., L.K. Ayliffe, L.K. Fifield, C.S.M. Turney, R.G. Cresswell, T.T. Barrows and B. David 1999 Radiocarbon dating of "old" charcoal using a wet oxidation, stepped-combustion procedure. Radiocarbon 41(2):127-140.

Bird, M.K. 1987 Life's a Beach: A Preliminary Investigation of the Coastal Archaeology of Wunjunga (Beach Mount), Upstart Bay, North Queensland. Unpublished B.A. (Hons) thesis, Department of Behavioural Sciences, James Cook University of North Queensland, Townsville.

Birkett, C. 1983 Corridor of Karst and Creek: A Site Catchment Analysis of Walkunder Arch Cave, near Chillagoe, North Queensland. Unpublished B.A. (Hons) thesis, School of Behavioural Sciences, James Cook University of North Queensland, Townsville.

Birkett, C. 1985 A site catchment analysis: Walkunder Arch Cave, near Chillagoe, north Queensland. Queensland Archaeological Research 2:132-143.

Bonica, C. 1992 A Technological Analysis of the Lithic Assemblage from Christmas Creek Rockshelter, southeast Queensland. Unpublished B.A. (Hons) thesis, Department of Anthropology and Sociology, University of Queensland, Brisbane.

Border, A. 1993 Shoalwater Bay Heritage Military Training Area (SWBTA): A Review of Cultural Heritage Resources and Significance and Land Use. UQASU Report 235. Brisbane: University of Queensland Archaeological Services Unit, Department of Anthropology and Sociology, University of Queensland.

Border, A. 1994 Shoalwater Bay Military Training Area (SWBTA): A review of cultural heritage resources their significance and land use. In Commonwealth Commission of Inquiry Shoalwater Bay, Capricornia 
Coast, Queensland, Research Reports, pp.173-233. No. 5, Vol. A. Canberra: Australian Government Publishing Service.

Border, A. 1999 Aboriginal settlement of offshore islands in the southern Great Barrier Reef Province, central Queensland. In J. Hall and I.J. McNiven (eds), Australian Coastal Archaeology, pp.129-139. Research Papers in Archaeology and Natural History 31. Canberra: ANH Publications, Department of Archaeology and Natural History, Research School of Pacific and Asian Studies, Australian National University.

Bowen, G. 1989 A Model for Moreton Island Prehistory: Colonisation, Settlement and Subsistence. Unpublished B.A. (Hons) thesis, Department of Anthropology and Sociology, University of Queensland, Brisbane.

Bowen, G. 1998 Towards a generic technique for dating stone fish traps and weirs. Australian Archaeology 47:39-43.

Brayshaw, H.C. 1975 Fieldwork in north Queensland. Australian Archaeology 2:48.

Brayshaw, H.C. 1977 Aboriginal Material Culture in the Herbert/Burdekin District, North Queensland. Unpublished $\mathrm{PhD}$ thesis, James Cook University of North Queensland, Townsville.

Brayshaw, H.C. 1990 Well Beaten Paths: Aborigines of the Herbert Burdekin District, North Queensland: An Ethnographic and Archaeological Study. Townsville: Department of History, James Cook University of North Queensland.

Breschini, G.S., T. Haversat and J. Erlandson 1996 California Radiocarbon Dates. $8^{\text {th }}$ ed. Salinas, CA: Coyote Press.

Brian, D. 1994 Shall I Compare Thee to a Fish?: A Comparative Taphonomic Analysis of Vertebrate Remains from Nara Inlet Art Site, Hook Island, Central Queensland Coast. Unpublished B.A. (Hons) thesis, Department of Anthropology and Sociology, University of Queensland, Brisbane.

Burke, C. 1988 Faunal Reflections of Change and Diversity: Monitoring the Effects of Natural and Cultural Site Formation Processes on the Composition of Archaeological Faunal Assemblages, a Case Study from Boonah, Southeast Queensland. Unpublished B.A. (Hons) thesis, Department of Archaeology and Palaeoanthropology, University of New England, Armidale.

Burke, C. 1993 A Survey of Aboriginal Archaeological Sites on the Curtis Coast, Central Queensland. Unpublished report to the Queensland Department of Environment and Heritage, Rockhampton.

Busuttin-Windsor, V. 1982 Island That We Knew: The Story of Brampton Island and the Pioneer Busuttin Family. Hong Kong: Bruwal Press.

Caddie, D.A., H.J. Hall, D.S. Hunter and P.J. Pomery 1984 The application of electron spin resonance dating to Holocene bone. Australian Archaeology 19:75-85.
Callow, W.J., M.J. Baker and G.I. Hassall 1965 National Physical Laboratory radiocarbon measurements III. Radiocarbon 7:156-161.

Callow, W.J., M.J. Baker and D.H. Pritchard 1963 National Physical Laboratory radiocarbon measurements I. Radiocarbon 5:34-38.

Campbell, J.B. 1978 Preliminary report on 1977 surveys and excavations at Turtle Rock, Hervey Range, north Queensland. Australian Archaeology 8:4-15.

Campbell, J.B. 1979 Settlement patterns on off-shore islands in north-eastern Queensland. Australian Archaeology 9:18-32.

Campbell, J.B. 1982a Automatic seafood retrieval systems: The evidence from Hinchinbrook Island and its implications. In S. Bowdler (ed.), Coastal Archaeology in Eastern Australia: Proceedings of the 1980 Valla Conference on Australian Prehistory, pp.96-107. Occasional Papers in Prehistory 11. Canberra: Department of Prehistory, Research School of Pacific Studies, Australian National University.

Campbell, J.B. 1982b New radiocarbon results for north Queensland prehistory. Australian Archaeology 14:6266.

Campbell, J.B. 1984 Extending the archaeological frontier: A review of work on the prehistory of north Queensland. Queensland Archaeological Research $1: 173-184$.

Campbell, J.B. 2000 The Chillagoe and Laura laser-AMS dating project. In G.K. Ward and C. Tuniz (eds), Advances in Dating Australian Rock-Markings: Papers from the First Australian Rock-Picture Dating Workshop, pp.80-83. Occasional AURA Publication 10. Melbourne: Australian Rock Art Research Association.

Campbell, J., N. Cole, E. Hatte, C. Tuniz and A. Watchman 1996 Dating of rock surface accretions with Aboriginal paintings and engravings in north Queensland. In S. Ulm, I. Lilley and A. Ross (eds), Australian Archaeology '95: Proceedings of the 1995 Australian Archaeological Association Annual Conference, pp.231-239. Tempus 6. St Lucia: Anthropology Museum, Department of Anthropology and Sociology, University of Queensland.

Campbell, J.B. and M. Mardaga-Campbell 1993 From macro- to nano-stratigraphy: Linking vertical and horizontal dating of archaeological deposits with the direct dating of rock art at 'The Walkunders', Chillagoe (north Queensland, Australia). In J. Steinbring, A. Watchman, P. Faulstich and P. Taçon (eds), Time and Space: Dating and Spatial Considerations in Rock Art Research, pp.57-63. Occasional AURA Publication 8. Melbourne: Australian Rock Art Research Association.

Carter, M. 1997 Chenier and Shell Midden: An Investigation of Cultural and Natural Shell Deposits at Rodds Peninsula, Central Queensland Coast. Unpublished B.A. (Hons) thesis, Department of Anthropology and Sociology, University of Queensland, Brisbane. 
Carter, M. 1999 Thesis Abstract: Chenier and Shell Midden: An Investigation of Cultural and Natural Shell Deposits at Rodds Peninsula, Central Queensland Coast. Australian Archaeology 48:56.

Carter, M., I. Lilley, S. Ulm and D. Brian 1999 Mort Creek Site Complex, Curtis Coast: Site report. Queensland Archaeological Research 11:85-104.

Chapman, V. 1999 Drawing the Line: The Rock Painting of Cania Gorge, South Central Queensland. Unpublished PGDipArts (Anthropology) thesis, Department of Sociology, Anthropology and Archaeology, University of Queensland, Brisbane.

Chicoteau, C. 1998a Two Hundred and Four Kilograms of What?: A Preliminary Site Report of Square A, Grinding Groove Cave, Cania Gorge, Focussing on Sedimentation Rates. Unpublished report submitted for ID232: Independent Project in Aboriginal and Torres Strait Islander Studies I, Aboriginal and Torres Strait Islander Studies Unit, University of Queensland, Brisbane.

Chicoteau, C. 1998b An Analysis of Archaeological Charcoals from Square A, Grinding Groove Cave, Cania Gorge: A Feasibility Study of the Use of Archaeological Charcoals in Cania Gorge Rockshelter Deposits. Unpublished report submitted for ID233: Independent Project in Aboriginal and Torres Strait Islander Studies II, Aboriginal and Torres Strait Islander Studies Unit, University of Queensland, Brisbane.

Chillagoe Caving Club 1988 Mitchell-Palmer Karst: A Speleological Field Guide for the Towers and Caves of the Mitchell-Palmer Areas in Far North Queensland, Australia. Cairns: Chillagoe Caving Club Inc.

Clegg, J.F. 1965 A note on the stone industry of Cathedral Cave, Carnarvon Gorge, Queensland. Mankind 6(5):237.

Clegg, J.K. 1977 The four dimensions of artifactual variation. In R.V.S. Wright (ed.), Stone Tools as Cultural Markers: Change, Evolution and Complexity, pp.60-66. Prehistory and Material Culture Series 12. Canberra: Australian Institute of Aboriginal Studies.

Clouten, N. 1976 Photogrammetry and the measurement of rock form and Aboriginal art. Australian Institute of Aboriginal Studies Newsletter (N.S.) 6:33-45.

Clouten, N. 1977 Further photogrammetric recordings of Early Man shelters, Cape York. Australian Institute of Aboriginal Studies Newsletter (N.S.) 7:54-59.

Cole, N.A. 1998 Eel and Boomerang: An Archaeological Study of Stylistic Order and Variability in Aboriginal Rock Art of the Laura Sandstone Province, Cape York Peninsula, Australia. Unpublished PhD thesis, School of Anthropology and Archaeology, James Cook University of North Queensland, Townsville.

Cole, N. 2000 The 'direct dating' of rock-pictures at Laura. In G.K. Ward and C. Tuniz (eds), Advances in Dating Australian Rock-Markings: Papers from the First Australian Rock-Picture Dating Workshop, pp.76-79. Occasional AURA Publication 10. Melbourne:
Australian Rock Art Research Association.

Cole, N. and A. Watchman 1992 Painting with plants: Investigating fibres in Aboriginal rock paintings at Laura, north Queensland. Rock Art Research 9(1):2736.

Cole, N.A., A. Watchman and M.J. Morwood 1993 Evidence of a 25,000 year old pictograph from northern Australia. Geoarchaeology 8(6):465-473.

Cole, N.A., A. Watchman and M.J. Morwood 1995 Chronology of Laura rock art. In M.J. Morwood and D.R. Hobbs (eds), Quinkan Prehistory: The Archaeology of Aboriginal Art in S.E. Cape York Peninsula, Australia, pp.147-160. Tempus 3. St Lucia: Anthropology Museum, Department of Anthropology and Sociology, University of Queensland.

Colfelt, D. and D. Bradley 1980 The Complete Yachtsman's Handbook to the Whitsunday Passage. Crows Nest: Whitsunday World Pty Ltd.

Collier, S. and K.A. Hobson 1987 The importance of marine protein in the diet of coastal Australian Aborigines. Current Anthropology 28(4):559-565.

Cook, N.D.J., I. Davidson and S.A. Sutton 1989 Seeing red in Queensland. Nature 342:487.

Cook, N.D.J., I. Davidson and S.A. Sutton 1990 Why are so many ancient rock paintings red? Australian Aboriginal Studies 1990(1):30-32.

Cosgrove, R. 2000 Australian Tropical Rainforest Archaeology. Unpublished paper presented to the Australian Archaeological Association Annual Conference, Beechworth, Victoria, 2 December 2000.

Coventry, R.J., D. Hopley, J.B. Campbell, I. Douglas, N. Harvey, A.P. Kershaw, J. Oliver, C.V.G. Phipps and K. Pye 1980 The Quaternary of northeastern Australia. In R.A. Henderson and P.J. Stephenson (eds), The Geology and Geophysics of North Eastern Australia, pp.375-417. Brisbane: Geological Society of Australia, Queensland Division.

Crooks, J. 1982 Report of Three Excavations at Pumicestone Passage, Moreton Bay, Southeast Queensland. Unpublished B.A. (Hons) thesis, Department of Anthropology and Sociology, University of Queensland, Brisbane.

Dagg, L. 1991 A Comparative Faunal Analysis of Red Bluff and Red Horse Rockshelters, S.E. Cape York Peninsula. Unpublished paper to the Department of Archaeology and Palaeoanthropology, University of New England, Armidale.

Dagg, L. 1992 Interpreting Faunal Remains: A Taphonomic Analysis of Two Late Holocene Faunal Assemblages from South East Cape York Peninsula. Unpublished B.A. (Hons) thesis, Department of Archaeology and Palaeoanthropology, University of New England, Armidale.

David, B. 1983 To Pick a Bone: Differentiating between Cultural and Dingo-Accumulated Bone Debris - The Case of Walkunder Arch Cave. Unpublished B.A. (Hons) thesis, Department of Prehistory and 
Anthropology, Australian National University, Canberra.

David, B. 1984a Man vs. Dingo: The Identification of Bone Remains from Archaeological Sites, with Specific Reference to Walkunder Arch Cave, Chillagoe, Northeastern Queensland. Cultural Resource Management Monograph Series 5. Brisbane: Archaeology Branch, Department of Aboriginal and Islander Affairs.

David, B. 1984b Walkunder Arch Cave: A faunal report. Australian Archaeology 18:40-54.

David, B. 1987 Chillagoe: From Archaeology to Prehistory: Contributions to a Late Holocene Prehistory of the Chillagoe Region, North Queensland. Unpublished MA thesis, Australian National University, Canberra.

David, B. 1988 The Rock Pictures of the ChillagoeMungana and Mitchell-Palmer Limestone Belts of North Queensland: Reconstructing Cultural Relations in North Queensland's Prehistoric Past. Unpublished report to the Australian Heritage Commission, Canberra.

David, B. 1989 Prehistoric rock art of southern Cape York Peninsula. Rock Art Research 6(2):153-155.

David, B. 1990a New dates from Fern Cave, Cape York Peninsula. Rock Art Research 7(1):74-75.

David, B. 1990b Echidna's Rest, Chillagoe: A site report. Queensland Archaeological Research 7:73-94.

David, B. 1991a Fern Cave, rock art and social formations: Rock art regionalisation and demographic models in southeastern Cape York Peninsula. Archaeology in Oceania 26(2):41-57.

David, B. 1991b Mitchell River Cave: A late PleistoceneHolocene sequence from southeastern Cape York Peninsula. Australian Aboriginal Studies 1991(2):6772.

David, B. 1992a Initial radiocarbon determinations from Nurrabullgin. Australian Aboriginal Studies 1992(1):77-79.

David, B. 1992b An AMS date for north Queensland rock art. Rock Art Research 9(2):139-141.

David, B. 1992c Recent research in southeast Cape York Peninsula: Nurrabullgin and Mordor Cave. Queensland Archaeological Research 9:50-53.

David, B. 1993 Nurrabullgin Cave: Preliminary results from a pre-37,000 year old rockshelter, north Queensland. Archaeology in Oceania 28(1):50-54.

David, B. 1994 A Space-Time Odyssey: Rock Art and Regionalisation in North Queensland Prehistory. Unpublished PhD thesis, Department of Anthropology and Sociology, University of Queensland, Brisbane.

David, B. (ed.) 1998 Ngarrabullgan: Geographical Investigations in Djungan Country, Cape York Peninsula. Monash Publications in Geography and Environmental Science 51. Clayton, VIC: Department of Geography and Environmental Science, Monash University.
David, B., R.A. Armitage, M. Hyman, M.W. Rowe and E. Lawson 1999 How old is north Queensland's rock-art?: A review of the evidence, with new AMS determinations. Archaeology in Oceania 34(3):103120.

David, B., R. Bird, R. Fullagar and L. Little 1992 Glassy obsidian artefacts from north Queensland: The Nolan's Creek source and some archaeological occurrences. The Artefact 15:25-30.

David, B. and D. Chant 1995 Rock art and regionalisation in north Queensland prehistory. Memoirs of the Queensland Museum 37(2):357-528.

David, B., E. Clayton and A. Watchman 1993 Initial results of PIXE analysis on northern Queensland ochres. Australian Archaeology 36:50-57.

David, B. and L. Dagg 1993 Two caves. Memoirs of the Queensland Museum 33(1):143-162.

David, B. and H. Lourandos 1997 37,000 years and more in tropical Australia: Investigating long-term archaeological trends in Cape York Peninsula. Proceedings of the Prehistoric Society 63:1-23.

David, B. and H. Lourandos 1998 Rock art and sociodemography in northeastern Australian prehistory. World Archaeology 30(2):193-219.

David, B. and H. Lourandos 1999 Landscape as mind: Land use, cultural space and change in north Queensland prehistory. Quaternary International 59:107-123.

David, B., I. McNiven, L. Bekessy, B. Bultitude, C. Clarkson, E. Lawson, C. Murray and C. Tuniz 1998 More than 37,000 years of Aboriginal occupation. In B. David (ed.), Ngarrabullgan: Geographical Investigations in Djungan Country, Cape York Peninsula, pp.157-178. Monash Publications in Geography and Environmental Science 51. Clayton, VIC: Department of Geography and Environmental Science, Monash University.

David, B., R. Roberts, C. Tuniz, R. Jones and J. Head 1997 New optical and radiocarbon dates from Ngarrabullgan Cave, a Pleistocene archaeological site in Australia: Implications for the comparability of time clocks and for the human colonization of Australia. Antiquity $71: 183-188$.

David, B., M. Rowe, C. Tuniz and J. Head 1994 Dating charcoal paintings and drawings from Chillagoe: Current research. Rock Art Research 11(2):127-128.

David, B. and J. Stanisic 1991 Land snails in Australian archaeology: Initial results from Echidna's Rest (north Queensland). The Artefact 14:19-24.

David, B., C. Tuniz, E. Lawson, Q. Hua, G.E. Jacobsen, J. Head and M. Rowe 2000 Dating charcoal drawings from Chillagoe, north Queensland. In G.K. Ward and C. Tuniz (eds), Advances in Dating Australian RockMarkings: Papers from the First Australian RockPicture Dating Workshop, pp.85-89. Occasional AURA Publication 10. Melbourne: Australian Rock Art Research Association. 
David, B. and M. Wilson 1999 Re-reading the landscape: Place and identity in NE Australia during the late Holocene. Cambridge Archaeological Journal 9(2):163-188.

David, T.W.E. and J.T. Wilson 1914a Preliminary communication on an Australian cranium of probable Pleistocene age. Reports of the British Association for the Advancement of Science p.531.

David, T.W.E. and J.J. Wilson 1914b The Talgai Skull. The Scientific Australian September:4-5.

Davidson, D.S. 1936 Aboriginal Australian and Tasmanian rock carvings and paintings. Memoirs of the American Philosophical Society 5:1-151.

Davidson, I. 1993 Archaeology in the Selwyn Ranges. In G. Burenhult (ed.), People of the Stone Age: HunterGatherers and Early Farmers, pp.210-211. Illustrated History of Humankind 2. St Lucia: University of Queensland Press.

Davidson, I., S.A. Sutton and S.J. Gale 1993 The human occupation of Cuckadoo 1 Rockshelter, northwest central Queensland. In M.A. Smith, M. Spriggs and B. Fankhauser (eds), Sahul in Review: Pleistocene Archaeology in Australia, New Guinea and Island Melanesia, pp.164-172. Occasional Papers in Prehistory 24. Canberra: Department of Prehistory, Research School of Pacific Studies, Australian National University.

Donoghue, D. 1979 A Procedure for Identifying Archaeological Charcoal: An Example for Moreton Island, Southeast Queensland. Unpublished BA (Hons) thesis, Department of Anthropology and Sociology, University of Queensland, Brisbane.

Eales, T. 1998 Stone Soup: A Residue Analysis of Artefacts from Roof Fall Cave, Cania Gorge, Central Queensland. Unpublished B.A. (Hons) thesis, Department of Anthropology and Sociology, University of Queensland, Brisbane.

Eales, A. 1999 Thesis Abstract: Stone Soup: A Residue Analysis of Artefacts from Roof Fall Cave, Cania Gorge, Central Queensland. Australian Archaeology 48:55.

Eales, T., C. Westcott, I. Lilley, S. Ulm, D. Brian and C. Clarkson 1999 Roof Fall Cave, Cania Gorge: Site report. Queensland Archaeological Research 11:29-42.

Edmonds, V.J. 1986 Subtropical Rainforest: Archaeological Evidence for Aboriginal Use in the Border Ranges, Southeast Queensland. Unpublished M.Litt thesis, Department of Archaeology and Palaeoanthropology, University of New England, Armidale.

Elkin, A.P. 1949 The origin and interpretation of petroglyphs in southeast Australia. Oceania 20(2):119157.

Elvery, M. 1995 A Radiographic Study of the Broadbeach Aboriginal Dentition. Unpublished BDentSt thesis, Department of Dentistry, University of Queensland, Brisbane.
Elvery, M.W., N.W. Savage and W.B. Wood 1998 Radiographic study of the Broadbeach Aboriginal dentition. American Journal of Physical Anthropology 107:211-219.

Firinu, G. 1999 Assessing Stratigraphic Integrity with Conjoin Sets at Roof Fall Cave, Cania Gorge. Unpublished report submitted for ID233: Independent Project in Aboriginal and Torres Strait Islander Studies II, Aboriginal and Torres Strait Islander Studies Unit, University of Queensland, Brisbane.

Flood, J. 1983 Quinkan country: The archaeology and art of Cape York Peninsula. Hemisphere 27:315-320.

Flood, J. 1987 Rock art of the Koolburra Plateau, north Queensland. Rock Art Research 4(2):91-126.

Flood, J. 1999 Archaeology of the Dreamtime: The Story of Prehistoric Australia and its People. Rev. ed. Sydney: Angus and Robertson.

Flood, J. and N. Horsfall 1986 Excavations of Green Ant and Echidna Shelters, Cape York Peninsula. Queensland Archaeological Research 3:4-64.

Flood, P.G. 1981 Carbon-14 dates from the coastal plains of Deception Bay, southeastern Queensland. Queensland Government Mining Journal 82:19-23.

Francis, V. 1999 Residue Analysis of Stone Artefacts from Open Shell Midden Sites on the Southern Curtis Coast. Unpublished report submitted for ID233: Independent Project in Aboriginal and Torres Strait Islander Studies II, Aboriginal and Torres Strait Islander Studies Unit, University of Queensland, Brisbane.

Francis, V. 2000 What's the Point: An Investigation of the Bone Artefacts from Platypus Rockshelter, Southeast Queensland. Unpublished B.A. (Hons) thesis, Department of Sociology, Archaeology and Anthropology, University of Queensland, Brisbane.

Frankel, D. 1982 Population trends and relationships in prehistoric Australia. Journal of Australian Studies 11:3-8.

Frankland, K. 1990 Booral: A Preliminary Investigation of an Archaeological Site in the Great Sandy Strait Region, Southeast Queensland. Unpublished B.A. (Hons) thesis, Department of Anthropology and Sociology, University of Queensland, Brisbane.

Freedman, L. and W.B. Wood 1977a The Broadbeach burial site, southeast Queensland: A metrical analysis of a cranial sample. Journal of Anatomy 124:150.

Freedman, L. and W.B. Wood 1977b Metrical features of Australian Aboriginal crania and mandibles from Broadbeach, southeast Queensland. Archaeology and Physical Anthropology in Oceania 12(1):1-25.

Fullagar, R. and B. David 1997 Investigating changing attitudes towards an Australian Aboriginal Dreaming mountain over $>37,000$ years of occupation via residue and use wear analyses of stone artefacts. Cambridge Archaeological Journal 7(1):139-144.

Ghaleb, B. 1990 An Ethnoarchaeological Study of Mabuiag Island, Torres Strait, Northern Australia. Unpublished $\mathrm{PhD}$ thesis, Institute of Archaeology, 
University College London, London.

Ghaleb, B. 1998 Fish and fishing on a western Torres Strait Island, northern Australia: Ethnographic and archaeological perspectives. Internet Archaeology 4. http://intarch.ac.uk/journal/issue4/fish/ghaleb/index.h $\underline{\mathrm{tml}}$

Gill, E.D. 1978a Age and origin of the Talgai cranium from the Darling Downs of Queensland, Australia. The Artefact 3(4):163-180.

Gill, E.D. 1978b Geology of the Late Pleistocene Talgai cranium from S.E. Queensland, Australia. Archaeology and Physical Anthropology in Oceania 13(2\&3):177197.

Gillespie, R. and H.A. Polach 1979 The suitability of marine shells for radiocarbon dating of Australian prehistory. In R. Berger and H. Suess (eds), Radiocarbon Dating: Proceedings of the $9^{\text {th }}$ International Conference on Radiocarbon Dating, pp.404-421. Los Angeles: University of California Press.

Gillespie, R. and R.B. Temple 1976 Sydney University natural radiocarbon measurements III. Radiocarbon 18(1):96-109.

Gillespie, R. and R.B. Temple 1977 Radiocarbon dating of shell middens. Archaeology and Physical Anthropology in Oceania 12(1):26-37.

Gillespie, R. and R.B. Temple 1979 Sydney University natural radiocarbon measurements $\mathrm{V}$. Radiocarbon 21(1):95-106.

Gillieson, D.S. 1982 Aboriginal Sites of the Albert Valley, South East Queensland. Cultural Resource Management Monograph Series 3. Brisbane: Archaeology Branch, Department of Aboriginal and Islanders Advancement.

Gillieson, D. 1984 Geoarchaeological applications of scanning electron microscopy: Some Australian examples. The Artefact 6:43-54.

Gillieson, D.S. and J. Hall 1982 Bevelling bungwall bashers: A use-wear study from southeast Queensland. Australian Archaeology 14:43-61.

Goddard, R.H. 1940-1941 Aboriginal rock sculpture and stencilling in the Carnarvon Ranges. Oceania 11:368372.

Godwin, L. 1985 The Gyranda Weir Archaeological Salvage Project: A Report on the Analysis of Sites 1, 2, 3, 5, 7, and 8. Unpublished report to the Queensland Water Resources Commission, Brisbane.

Godwin, L. 1990 Cultural heritage. In J. McCosker, Eurimbula National Park Draft Management Plan. Unpublished report to the Queensland Department of Environment and Heritage, Rockhampton.

Goodall, R., B. David and J. Bartley 1996 Non-destructive techniques for the analysis and characterisation of pigments from archaeological sites: The case of Fern Cave. In S. Ulm, I. Lilley and A. Ross (eds), Australian Archaeology '95: Proceedings of the 1995 Australian Archaeological Association Annual Conference, pp.183-187. Tempus 6. St Lucia: Anthropology Museum, Department of Anthropology and Sociology, University of Queensland.

Gould, R.A. 1978 Beyond analogy in ethnoarchaeology. In R.A. Gould (ed.), Explorations in Ethnoarchaeology, pp.249-321. Albuquerque: University of New Mexico Press.

Gowlett, J.A.J., R.E.M. Hedges, I.A. Law and C. Perry 1987 Radiocarbon dates from the Oxford AMS system: Archaeometry datelist 5. Archaeometry 29(1):125-155.

Grainer, J., B. David, R. Cribb, B. White and H. Kuhn 1992 Nurrabullgin - 'A mountain, once seen, never to be forgotten'. Rock Art Research 9(1):74-77.

Gunn, B. and A. Thorn 1994 Aboriginal Rock Art in the Wet Tropics World Heritage Area, Northern Queensland: A Recording and Conservation Assessment. Unpublished report to Department of Environment and Heritage, Cairns.

Haddon, A.C. (ed.) 1904 Sociology, Magic and Religion of the Western Islanders. Reports of the Cambridge Anthropological Expedition to Torres Straits 5. Cambridge: Cambridge University Press.

Haglund, L. 1974 The Sandstone Point Shell Midden: A Preliminary Report. Appendix 5. In J. Stockton, Report of an Archaeological Survey in the Vicinity of Bribie Island, South-East Queensland, pp.118-131. Unpublished B.A. (Hons) thesis, Department of Anthropology and Sociology, University of Queensland, Brisbane.

Haglund, L. 1976 The Broadbeach Aboriginal Burial Ground: An Archaeological Analysis. St Lucia: University of Queensland Press.

Haglund-Calley, L. 1968 An Aboriginal burial ground at Broadbeach, Queensland: Excavation report. Mankind 6(12):676-680.

Haglund-Calley, L. 1969 The Relation between the Broadbeach Burials and the Cultures of Eastern Australia. Unpublished MA thesis, Department of Anthropology and Sociology, University of Queensland, Brisbane.

Haglund-Calley, L. and M.C. Quinnell 1973 A shell midden at Cascade Gardens, Broadbeach, southeast Queensland. Memoirs of the Queensland Museum 16(3):399-409.

Hale, H.M. and N.B. Tindale 1933 Aborigines of Princess Charlotte Bay. Records of the South Australian Museum 5:118-162.

Hall, H.J. 1980a Archaeology at the University of Queensland: A brief outline. Australian Archaeology 10:79-85.

Hall, H.J. 1980b Minner Dint: A recent Aboriginal midden on Moreton Island, southeastern Queensland. In P.K. Lauer (ed.), Occasional Papers in Anthropology 10:94112. St Lucia: Anthropology Museum, University of Queensland.

Hall, H.J. 1982 Sitting on the crop of the Bay: An historical and archaeological sketch of Aboriginal 
settlement and subsistence in Moreton Bay, southeast Queensland. In S. Bowdler (ed.), Coastal Archaeology in Eastern Australia: Proceedings of the 1980 Valla Conference on Australian Prehistory, pp.79-95. Occasional Papers in Prehistory 11. Canberra: Department of Prehistory, Research School of Pacific Studies, Australian National University.

Hall, H.J. 1984 Exploratory excavation at Toulkerrie midden (LB:B75), Moreton Island, S.E. Queensland. Queensland Archaeological Research 1:61-84.

Hall, J. 1986 Exploratory excavation at Bushrangers Cave (Site LA:A11), a 6000-year-old campsite in southeast Queensland: Preliminary results. Australian Archaeology 22:88-103.

Hall, H.J. 1987 A short prehistory of the Moreton Region. In R. Fisher (ed.), Brisbane: Aboriginal, Alien, Ethnic, pp.14-22. Brisbane History Group Papers 5. St Lucia: Department of History, University of Queensland.

Hall, J. 1988a An Archaeological Inspection of Proposed Alternate Route for Existing Road at Oysterman's Reserve, Toulkerrie, Moreton Island. UQASU Report 125. Brisbane: University of Queensland Archaeological Services Unit, Department of Anthropology and Sociology, University of Queensland.

Hall, J. 1988b An Archaeological Management Plan for the Proposed Oysterman's Reserve, Toulkerrie, Moreton Island. UQASU Report 127. Brisbane: University of Queensland Archaeological Services Unit, Department of Anthropology and Sociology, University of Queensland.

Hall, H.J. 199020000 years of human impact on the Brisbane River and environs. In P. Davie, E. Stock and D.L. Choy (eds), The Brisbane River: A Source-Book for the Future, pp.175-182. Brisbane: Australian Littoral Society and the Queensland Museum.

Hall, H.J. 1995 The Saint-Smith Midden Site at Beachmere, S.E. Queensland: A Report on Archaeological Fieldwork 1994. Unpublished report to the Heritage Section, Queensland Department of Environment and Heritage. St Lucia: Department of Anthropology and Sociology, University of Queensland.

Hall, H.J. 1999 The impact of sea level rise on the archaeological record of the Moreton region, southeast Queensland. In J. Hall and I.J. McNiven (eds), Australian Coastal Archaeology, pp.169-184. Research Papers in Archaeology and Natural History 31. Canberra: ANH Publications, Department of Archaeology and Natural History, Research School of Pacific and Asian Studies, Australian National University.

Hall, J. and G. Bowen 1989 An excavation of a midden complex at the Toulkerrie Oysterman's lease, Moreton Island, SE Queensland. Queensland Archaeological Research 6:3-27.

Hall, J. and G. Bowen 1990 Excavation of the Midden Complex at the Toulkerrie Oysterman's Lease, Moreton
Island, S.E. Queensland: Significance and Management. UQASU Report 148. Brisbane: University of Queensland Archaeological Services Unit, Department of Anthropology and Sociology, University of Queensland.

Hall, J. and P. Hiscock 1988 The Moreton Regional Archaeological Project (MRAP) - Stage II: An outline of objectives and methods. Queensland Archaeological Research 5:4-24.

Hall, J. and I. Lilley 1987 Excavation at the New Brisbane Airport site (LB:C69): Evidence for early midHolocene coastal occupation in Moreton Bay, SE Queensland. Queensland Archaeological Research 4:54-79.

Hekel, H., W.T. Ward, M. Jones and D.E. Searle 1979 Geological development of northern Moreton Bay. In A. Bailey and N.C. Sevens (eds), Northern Moreton Bay Symposium, pp.7-18. Brisbane: Royal Society of Queensland.

Helman, M. 1934 The form of the Talgai palate. American Journal of Physical Anthropology 19:1-15.

Hendy, C.H., T.A. Rafter and N.W.G. Macintosh 1973 The formation of carbonate nodules in the soils of the Darling Downs, Queensland, Australia and the dating of the Talgai cranium. In Proceedings of the $8^{\text {th }}$ International Conference on Radio Carbon Dating, Wellington, New Zealand, pp.D106-126.

Hermes, M. 1984 Mazie Bay: An Analysis and Interpretation of the Faunal Component. Unpublished B.A. (Hons) thesis, Department of Prehistory and Anthropology, Australian National University, Canberra.

Higham, T. 2000 Age calculation. Radiocarbon Web-Info. http://www.c14dating.com/agecalc.html

Hiscock, P. 1984 A preliminary report on the stone artefacts from Colless Creek cave, northwest Queensland. Queensland Archaeological Research 1:120-151.

Hiscock, P. 1985 The need for a taphonomic perspective in stone artefact analysis. Queensland Archaeological Research 2:82-97.

Hiscock, P. 1988a Prehistoric Settlement Patterns and Artefact Manufacture at Lawn Hill, Northwest Queensland. Unpublished PhD thesis, Department of Anthropology and Sociology, University of Queensland, Brisbane.

Hiscock, P. 1988b Developing a relative dating system for the Moreton Region: An assessment of prospects for a technological approach. Queensland Archaeological Research 5:113-132.

Hiscock P. 1990 A study in scarlet: Taphonomy and inorganic artefacts. In S. Solomon, I. Davidson and D. Watson (eds), Problem Solving in Taphonomy: Archaeological and Palaeontological Studies from Europe, Africa and Oceania, pp.34-49. Tempus 2. St Lucia: Anthropology Museum, Department of Anthropology and Sociology, University of Queensland. 
Hiscock, P. and J. Hall 1988a Technological change at Platypus Rockshelter (KB:A70), southeast Queensland. Queensland Archaeological Research 5:63-89.

Hiscock, P. and J. Hall 1988b Technological change at Bushranger's Cave (LA:A11) S.E. Queensland. Queensland Archaeological Research 5:90-112.

Hiscock, P. and P.J. Hughes 1980 Backed blades in northern Australia: Evidence from northwest Queensland. Australian Archaeology 10:86-95.

Hobson, K.A. and S. Collier 1984 Marine and terrestrial protein in Australian Aboriginal diets. Current Anthropology 25(2):238-240.

Hobson, K.A. and S. Collier 1986 On Australian Aboriginal diets: Reply. Current Anthropology 27(1):54.

Holden, A. 1999 A Technological Analysis of the Lithic Assemblage from Hay Cave, S.E. Cape York Peninsula: Considering Diachronic Variations in Patterns of 'Intensity of Site Use'. 2 vols. Unpublished B.A. (Hons) thesis, Department of Sociology, Anthropology and Archaeology, University of Queensland, Brisbane.

Hopley, D. 1970a Coastal Geomorphology in the Townsville Region. Unpublished $\mathrm{PhD}$ thesis, James Cook University of North Queensland, Townsville.

Hopley, D. 1970b The Geomorphology of the Burdekin Delta, North Queensland. Monograph Series 1. Townsville: Department of Geography, James Cook University of North Queensland.

Horsfall, N. 1983 Excavations at Jiyer Cave, northeast Queensland: Some results. In M. Smith (ed.), Archaeology at ANZAAS 1983, pp.172-178. Perth: Western Australian Museum.

Horsfall, N. 1987 Living in Rainforest: The Prehistoric Occupation of North Queensland's Humid Tropics. Unpublished PhD thesis, Department of Behavioural Science, James Cook University of North Queensland, Townsville.

Horsfall, N. 1996 Holocene occupation of the tropical rainforests of north Queensland. In P. Veth and P. Hiscock (eds), Archaeology of Northern Australia, pp.175-190. Tempus 4. St Lucia: Anthropology Museum, Department of Anthropology and Sociology, University of Queensland.

Horton, D.R. 1977 A 10,000 year old Sarcophilus from Cape York. Search 8:374-375.

Hubbs, C.L., G.S. Bien and H.E. Suess 1965 La Jolla natural radiocarbon measurements IV. Radiocarbon 7:66-117.

Huchet, B.M.J. 1990 A taphonomic analysis of the faunal assemblage from Yam Camp rockshelter, S.E. Cape York Peninsula. Queensland Archaeological Research 7:57-72.

Hughes, P.J. 1983 Colless Creek rock shelter archaeological site, northwest Queensland - 18KA. In J.M.A. Chappell and A. Grindrod (eds), Proceedings of the First CLIMANZ Conference, Victoria, Feb 8-13,
1981, pp.59-61. Canberra: Department of Biogeography and Geomorphology, Research School of Pacific Studies, Australian National University.

Johnston, H. and M. Rowland 1987 Representativeness, Conservation and the Archaeology Branch (Queensland) Site Register. Unpublished report to the Archaeology Branch, Department of Community Services, Brisbane.

Jung, S. 1990 Chronological Changes in Stone Artefact Resource Utilisation at Magnificent Gallery, South-East Cape York Peninsula, Northern Queensland. Unpublished M.Litt thesis, Department of Archaeology and Palaeoanthropology, University of New England, Armidale.

Jung, S. 1992 Trample damage of stone flakes as an index of occupation intensity: A case study from Magnificent Gallery. Queensland Archaeological Research 9:26-28.

Kearney, A.J. 1998 Bushrangers Cave: An Archaeofaunal Analysis and Palaeoenvironmental Study. Unpublished B.A. (Hons) thesis, Department of Anthropology and Sociology, University of Queensland, Brisbane.

Kelly, M. 1982 A Practical Reference Source to Radiocarbon Dates Obtained from Archaeological Sites in Queensland. Cultural Resource Management Monograph Series 4. Brisbane: Archaeology Branch, Department of Aboriginal and Islanders Advancement.

Kigoshi, K. and H. Kobayashi 1966 Gakushuin natural radiocarbon measurements V. Radiocarbon 8:54-73.

Krueger, V.L. 1997 No Place Like Home: An Investigation of Site Use During the Last Glacial Maximum at Fern Cave, Northeastern Queensland. Unpublished B.A. (Hons) thesis, Department of Anthropology and Sociology, University of Queensland, Brisbane.

Lamb. L. 1993 Fern Cave: A Technological Investigation of Increased Stone Artefact Deposition Rates. Unpublished B.A. (Hons) thesis, Department of Anthropology and Sociology, University of Queensland, Brisbane.

Lamb, L. 1996 Investigating changing stone technologies, site use and occupational intensities at Fern Cave, north Queensland. Australian Archaeology 42:1-6.

Langham, I. 1978 Talgai and Piltdown - The common context. The Artefact 3(4):181-224.

Lauer, P.K. 1978 The museum's role in fieldwork: The Fraser Island study. In P.K. Lauer (ed.), Occasional Papers in Anthropology 9:31-72. St Lucia: Anthropology Museum, University of Queensland.

Lauer, P.K. 1986 Report and Recommendations on the Aboriginal Shell Midden at Polka Point, Stradbroke Island. Unpublished report to the Anthropology Museum, University of Queensland, Brisbane.

Lauer, P.K. and G.H. Dillon 1986 The Kombumerri Culture Centre: A progress report. Bulletin of the Conference of Museum Anthropologists 19:22-25.

Lavell, C., R.L. Otlet and A.J. Walker 1992 The CBA/RCD computer database of radiocarbon dated sites. Antiquity 66:969-974. 
Lee-Thorpe, J.A. and J.C. Sealy 1986 On Australian Aboriginal diets. Current Anthropology 27(1):54.

L'Estrange, S. 1998 Tainted Glass. Unpublished report submitted for ID233: Independent Project in Aboriginal and Torres Strait Islander Studies II, Aboriginal and Torres Strait Islander Studies Unit, University of Queensland, Brisbane.

Lilley, I.A. 1982 A Finger on the Pulse: Analysis of Site Location Patterns in Subcoastal Southeast Queensland. Unpublished MA thesis, Department of Anthropology and Sociology, University of Queensland, Brisbane.

Lilley, I. 1994 An Archaeological Assessment of Proposed Sand-Mining on Middle Island, Coastal Central Queensland. UQASU Report 244. Brisbane: University of Queensland Archaeological Services Unit, Department of Anthropology and Sociology, University of Queensland, Brisbane.

Lilley, I., D. Brian, C. Clarkson and S. Ulm 1998 Pleistocene Aboriginal occupation at Cania Gorge, central Queensland: Preliminary results of fieldwork. Archaeology in Oceania 33(1):28-31.

Lilley, I., D. Brian and S. Ulm 1999a The use of foraminifera in the identification and analysis of marine shell middens: A view from Australia. In M-J. Mountain and D. Bowdery (eds), Taphonomy: The Analysis of Processes from Phytoliths to Megafauna, pp.9-16. Research Papers in Archaeology and Natural History 30. Canberra: ANH Publications, Research School of Pacific and Asian Studies, Australian National University.

Lilley, I.A. and H.J. Hall 1988 An Archaeological Assessment of Northeastern Moreton Island. UQASU Report 126. Brisbane: University of Queensland Archaeological Services Unit, Department of Anthropology and Sociology, University of Queensland, Brisbane.

Lilley, I. and S. Ulm 1995 The Gooreng Gooreng Cultural Heritage Project: Some proposed directions and preliminary results of the archaeological program. Australian Archaeology 41:11-15.

Lilley, I. and S. Ulm 1999 The Gooreng Gooreng Cultural Heritage Project: Preliminary results of archaeological research, 1993-1997. Queensland Archaeological Research 11:1-14.

Lilley, I., S. Ulm and D. Brian 1996 The Gooreng Gooreng Cultural Heritage Project: First radiocarbon determinations. Australian Archaeology 43:38-40.

Lilley, I. S. Ulm and M. Williams (eds) 1999b The Gooreng Gooreng Cultural Heritage Project: Preliminary results of archaeological research, 19931997. Queensland Archaeological Research 11.

Lilley, I., M. Williams and S. Ulm 1997 The Gooreng Gooreng Cultural Heritage Project: A Report on National Estate Program Research, 1995-1996. 2 vols. Brisbane: Aboriginal and Torres Strait Islander Studies Unit, University of Queensland.

L'Oste-Brown, S. 1991 The East Side Story: A Tale of Technological Change in the Mid-to-Late Holocene.
Unpublished paper presented to the Department of Archaeology and Palaeoanthropology, University of New England, Armidale.

L'Oste-Brown, S. 1992 Getting Organised: Causes of Stone Artefact Variability in Southeast Cape York Peninsula. Unpublished B.A. (Hons) thesis, Department of Archaeology and Palaeoanthropology, University of New England, Armidale.

Macintosh, N.W.G. 1952 The Talgai teeth and dental arch: Remeasurement and reconstruction. Oceania 23(2):106-109.

Macintosh, N.W.G. 1965 The physical aspect of man in Australia. In R.M. Berndt and C.H. Berndt (eds), Aboriginal Man in Australia: Essays in Honour of Emeritus Professor A.P. Elkin, pp.46-50. Sydney: Angus and Robertson.

Macintosh, N.W.G. 1967a Fossil man in Australia: With particular reference to 1965 discovery at Green Gully near Keilor, Victoria. Australian Journal of Science 30(3):86-98.

Macintosh, N.W.G. 1967b Recent discoveries of early Australian man. Annals of the Australian College of Dental Surgeons 1:104-126.

Macintosh, N.W.G. 1969 The Talgai cranium: The value of archives. Australian Natural History 16:189-195.

Macintosh, N.W.G. 1973 Radiocarbon dating as a pointer in time to the arrival and history of man in Australia and islands to the north-west. In Proceedings of the $8^{\text {th }}$ International Conference on Radio Carbon Dating, Wellington, New Zealand, pp.XLIV-LVI.

Macintosh, N.W.G. 1974 Early man and the dog in Australia. In A.P. Elkin and N.W.G. Macintosh (eds), Grafton Elliot Smith: The Man and His Work, pp.8394. Sydney: Sydney University Press.

MacLellan, A.S. 1901 The Queenslander 9 February 1901:66.

Magee, J. 1994 Analysis of Carbonate Mineralogies, Fern Cave. Appendix D. In B. David, A Space-Time Odyssey: Rock Art and Regionalisation in North Queensland Prehistory, pp.337-339. Unpublished PhD thesis, Department of Anthropology and Sociology, University of Queensland, Brisbane.

Magee, J. and B. David 1994 Nitrogen and Sediment Thin Section Analyses, Fern Cave. Appendix C. In B. David, A Space-Time Odyssey: Rock Art and Regionalisation in North Queensland Prehistory, pp.331-336. Unpublished PhD thesis, Department of Anthropology and Sociology, University of Queensland, Brisbane.

Magee, J.W. and P.J. Hughes 1982 Thin section analysis and the geomorphic history of the Colless Creek archaeological site in northwestern Queensland. In W. Ambrose and P. Duerden (eds), Archaeometry: An Australasian Perspective, pp.120-128. Occasional Papers in Prehistory 11. Canberra: Department of Prehistory, Research School of Pacific Studies, Australian National University.

Mahony, D.J. 1943a The antiquity of man in Australia. 
Australian Journal of Science 6:90.

Mahony, D.J. 1943b The problem of antiquity of man in Australia. Memoirs of the National Museum, Melbourne 13:7-56.

Mardaga-Campbell, M. 1986 Prehistoric living-floors and evidence for them in north Queensland rockshelters. Australian Archaeology 23:42-61.

Mardaga-Campbell, M. 1995 Under the Boardwalk: 'Living-Floor' Research in Aboriginal Rockshelters of North Queensland (Australia). Unpublished PhD thesis, Department of Anthropology and Archaeology, James Cook University of North Queensland, Townsville.

Mardaga-Campbell, M. and J.B. Campbell 1985 Lithic occurrences and stratigraphic problems at Turtle Rock (Hervey Range), north Queensland. Queensland Archaeological Research 2:98-131.

Marshall, A.J. 1935 The Whitsunday Isles. Australian Museum Magazine 5:414-418.

Maynard, L. 1979 The archaeology of Australian Aboriginal art. In S.M. Mead (ed.), Exploring the Visual Art of Oceania: Australia, Melanesia, Micronesia, and Polynesia, pp.83-110. Honolulu: University of Hawaii Press.

McFadgen, B., T. Higham and R. Sparks 2000 New Zealand radiocarbon database for archaeology. Archaeology in New Zealand 43(4):297-300.

McNiven, I. 1985 An archaeological survey of the Cooloola region, S.E. Queensland. Queensland Archaeological Research 2:4-37.

McNiven, I.J. 1988 Brooyar Rockshelter: A late Holocene seasonal hunting camp from southeast Queensland. Queensland Archaeological Research 5:133-160.

McNiven, I.J. 1989 Aboriginal shell middens at the mouth of the Maroochy River, southeast Queensland. Queensland Archaeological Research 6:28-52.

McNiven, I.J. 1990a Prehistoric Aboriginal Settlement and Subsistence in the Cooloola Region, Coastal Southeast Queensland. Unpublished PhD thesis, Department of Anthropology and Sociology, University of Queensland, Brisbane.

McNiven, I.J. 1990b Blowout taphonomy: Non-cultural associations between faunal and stone artefact assemblages along the Cooloola coast, southeast Queensland. Australian Archaeology 31:67-74.

McNiven, I.J. 1991a Teewah Beach: New evidence for Holocene coastal occupation in coastal southeast Queensland. Australian Archaeology 33:14-27.

McNiven, I.J. 1991b Settlement and subsistence activities along Tin Can Bay, southeast Queensland. Queensland Archaeological Research 8:85-107.

McNiven, I.J. 1991c The Double Island Point Aboriginal burials, coastal southeast Queensland. Australian Archaeology 32:10-16.

McNiven, I.J. 1992a Shell middens and mobility: The use of off-site faunal remains, Queensland, Australia. Journal of Field Archaeology 19(4):495-508.
McNiven, I.J. 1992b Bevel-edged tools from coastal southeast Queensland. Antiquity 66:701-709.

McNiven, I. 1992c Sandblow sites in the Great Sandy Region, coastal southeast Queensland: Implications for models of late Holocene rainforest exploitation and settlement restructuring. Queensland Archaeological Research 9:1-16.

McNiven, I.J. 1993 Raw material proximity and beveledged tool use, Teewah Beach, southeast Queensland. Archaeology in Oceania 28(3):138-143.

McNiven, I. 1998 Aboriginal archaeology of the Corroboree Beach dune field, Fraser Island: Re-survey and re-assessment. Memoirs of the Queensland Museum, Cultural Heritage Series 1(1):1-22.

McNiven, I.J. 1999 Fissioning and regionalisation: The social dimensions of changes in Aboriginal use of the Great Sandy Region, southeast Queensland. In J. Hall and I.J. McNiven (eds), Australian Coastal Archaeology, pp.157-168. Research Papers in Archaeology and Natural History 31. Canberra: ANH Publications, Department of Archaeology and Natural History, Research School of Pacific and Asian Studies, Australian National University.

Mills, R.A. 1992 Aboriginal Occupation of Lizard Island. Unpublished M.Litt thesis, Department of Prehistoric and Historical Archaeology, University of Sydney, Sydney.

Mitchell, S.R. 1940-1941 A note on artefacts in the Carnarvon Ranges. Oceania 11(4):372-373.

Moore, D.R. 1974 The Australian-Papuan Frontier at Cape York: A Reconstruction of the Ethnography of the Peoples on Mainland Cape York and the Adjacent Torres Strait Islands, their Interaction with each other, and their Prehistory; Incorporating Unpublished Documentary Evidence and an Archaeological Survey of the Area. Unpublished PhD thesis, Department of Prehistory, University of New England, Armidale.

Moore, D.R. 1979 Islanders and Aborigines at Cape York: An Ethnographic Reconstruction based on the 18481850 'Rattlesnake' Journals of O.W. Brierly and Information he obtained from Barbara Thompson. Canberra: Australian Institute of Aboriginal Studies.

Mortlock, A.J., D. Price and G. Gardiner 1979 The discovery and preliminary thermoluminescence dating of two Aboriginal cave shelters in the Selwyn Ranges, Queensland. Australian Archaeology 9:82-86.

Morwood, M. 1978 Ken's Cave and the art of centralwestern Queensland. Australian Archaeology 8:22-31.

Morwood, M. 1979 Art and Stone: Towards a Prehistory of Central Western Queensland. 2 vols. Unpublished PhD thesis, Department of Archaeology and Anthropology, Faculty of Arts, Australian National University, Canberra.

Morwood, M.J. 1980 St Helena National Park. Unpublished report to the Archaeology Branch, Department of Aboriginal and Islander Advancement, Brisbane. 
Morwood, M.J. 1981 Archaeology of the Central Queensland Highlands: The stone component. Archaeology in Oceania 16(1):1-52.

Morwood, M.J. 1984 The prehistory of the Central Queensland Highlands. In F. Wendorf and A. Close (eds), Advances in World Archaeology 3:325-379. Orlando: Academic Press.

Morwood, M.J. 1985 Preliminary Archaeological Assessment: Proposed Gyranda Weir, Dawson River, Central Queensland. Unpublished report to the Queensland Water Resources Commission, Brisbane.

Morwood, M.J. 1986a The archaeology of art: Excavations at Maidenwell and Gatton Shelters, southeast Queensland. Queensland Archaeological Research 3:88-132.

Morwood, M.J. 1986b An Archaeological Assessment of Sites in Nathan Gorge, Gyranda Weir Study Area. Unpublished report to the Queensland Water Resources Commission, Brisbane.

Morwood, M.J. 1987 The archaeology of social complexity in south-east Queensland. Proceedings of the Prehistoric Society 53:337-350.

Morwood, M 1989a The archaeology of Aboriginal art in S.E. Cape York: A research proposal. Rock Art Research 6(1):71-72.

Morwood, M. 1989b The archaeology of Aboriginal art in S.E. Cape York: Preliminary report on the 1989 fieldwork. Rock Art Research 6(2):155-156.

Morwood, M. 1990a The prehistory of Aboriginal landuse on the upper Flinders River, north Queensland highlands. Queensland Archaeological Research 7:356.

Morwood, M.J. 1990b Preliminary Report on the 1989 Excavations at Yam Camp Rockshelter, Red Bluff Rockshelter, Magnificent Shelter and Sandy Creek Rockshelter. Unpublished report to the Quinkan Reserves Trust.

Morwood, M.J. 1990c Edge-ground axes in Pleistocene Greater Australia - More evidence from S.E. Cape York Peninsula: A reply to Sutton. Queensland Archaeological Research 7:110-114.

Morwood, M.J. 1993 Cause and effect: Pleistocene Aboriginal occupation in the Quinkan region, southeast Cape York Peninsula. In M.A. Smith, M. Spriggs and B. Fankhauser (eds), Sahul in Review: Pleistocene Archaeology in Australia, New Guinea and Island Melanesia, pp.173-179. Occasional Papers in Prehistory 24. Canberra: Department of Prehistory, Research School of Pacific Studies, Australian National University.

Morwood, M. 1994 Handy household hints for archaeological excavations at rock art sites. Rock Art Research 11(1):10-12.

Morwood, M. 1995a Excavations at Giant Horse. In M. Morwood and D. Hobbs (eds), Quinkan Prehistory: The Archaeology of Aboriginal Art in SE Cape York Peninsula, Australia, pp.101-106. Tempus 3. St Lucia:
Anthropology Museum, Department of Anthropology and Sociology, University of Queensland.

Morwood, M. 1995b Excavations at Red Bluff 1. In M. Morwood and D. Hobbs (eds), Quinkan Prehistory: The Archaeology of Aboriginal Art in SE Cape York Peninsula, Australia, pp.127-132. Tempus 3. St Lucia: Anthropology Museum, Department of Anthropology and Sociology, University of Queensland.

Morwood, M. and L. Dagg 1995 Excavations at Yam Camp. In M. Morwood and D. Hobbs (eds), Quinkan Prehistory: The Archaeology of Aboriginal Art in SE Cape York Peninsula, Australia, pp.107-115. Tempus 3. St Lucia: Anthropology Museum, Department of Anthropology and Sociology, University of Queensland.

Morwood, M.J. and L.M. Godwin 1982 Aboriginal sites in the Hughenden region, north Queensland highlands: Research prospects. Australian Archaeology 15:49-53.

Morwood, M.J. and L. Godwin 1987 Archaeology of the Gyranda region, Dawson River, central Queensland. Queensland Archaeological Research 4:96-114.

Morwood, M. and D. Hobbs (eds) 1995 Quinkan Prehistory: The Archaeology of Aboriginal Art in SE Cape York Peninsula, Australia. Tempus 3. St Lucia: Anthropology Museum, Department of Anthropology and Sociology, University of Queensland.

Morwood, M., D. Hobbs and D. Price 1995a Excavations at Sandy Creek 1 and 2. In M. Morwood and D. Hobbs (eds), Quinkan Prehistory: The Archaeology of Aboriginal Art in SE Cape York Peninsula, Australia, pp.71-92. Tempus 3. St Lucia: Anthropology Museum, Department of Anthropology and Sociology, University of Queensland.

Morwood, M. and S. Jung 1995 Excavations at Magnificent Gallery. In M. Morwood and D. Hobbs (eds), Quinkan Prehistory: The Archaeology of Aboriginal Art in SE Cape York Peninsula, Australia, pp.92-100. Tempus 3. St Lucia: Anthropology Museum, Department of Anthropology and Sociology, University of Queensland.

Morwood, M. and S. L'Oste-Brown 1995 Excavations at Red Horse. In M. Morwood and D. Hobbs (eds), Quinkan Prehistory: The Archaeology of Aboriginal Art in SE Cape York Peninsula, Australia, pp.116-126. Tempus 3. St Lucia: Anthropology Museum, Department of Anthropology and Sociology, University of Queensland.

Morwood, M., S. L'Oste-Brown and D. Price 1995b Excavations at Mushroom Rock. In M. Morwood and D. Hobbs (eds), Quinkan Prehistory: The Archaeology of Aboriginal Art in SE Cape York Peninsula, Australia, pp.133-146. Tempus 3. St Lucia: Anthropology Museum, Department of Anthropology and Sociology, University of Queensland.

Morwood, M.J. and P.J. Trezise 1989 Edge-ground axes in Pleistocene Greater Australia: New evidence from southeast Cape York Peninsula. Queensland Archaeological Research 6:77-90. 
Mowat, F. 1989 Bushrangers Cave: A Faunal Analysis. Unpublished B.A. (Hons) thesis, Department of Anthropology and Sociology, University of Queensland, Brisbane.

Mulvaney, D.J. 1962 Advancing frontiers in Australian archaeology. Oceania 33(2):135-138.

Mulvaney, D.J. 1964 The Pleistocene colonization of Australia. Antiquity 38:263-267.

Mulvaney, D.J. 1971 Kenniff Cave C14 dates (1971). In J. Mummery (ed.), Prehistory and Heritage: The Writings of John Mulvaney, pp.72-73. Occasional Papers in Prehistory 17. Canberra: Department of Prehistory, Research School of Pacific Studies, Australian National University.

Mulvaney, D.J. 1975 The Prehistory of Australia. Rev. ed. Ringwood, VIC: Penguin Books.

Mulvaney, D.J. and E.B. Joyce 1965 Archaeological and geomorphological investigations on Mt. Moffatt Station, Queensland, Australia. Proceedings of the Prehistoric Society 31:147-212.

Murphy, A.M.C. 1978 The Broadbeach and Coastal Adelaide Aboriginal Populations: A Metrical Analysis of the Femora and Tibiae. Unpublished BSc (Hons), Department of Anatomy, University of Queensland, Brisbane.

Murphy, A.M.C. 1991 Into a Kingdom of Bones: A Metrical Analysis of the Pectoral Girdles and Innominates of the Broadbeach Australian Aborigines and the New Zealand Polynesians. Unpublished PhD thesis, Department of Anatomy, University of Queensland, Brisbane.

Murphy, A.M.C. and W.B. Wood 1983 Lower limb proportions in the Australian Aborigine: A reexamination. Proceedings of the XV Pacific Science Congress 1:1972.

Murphy, A.M.C. and W.B. Wood 1986 Lower limb proportions in the Australian Aborigine: Reexamination and Australasian perspectives. In G.K. Ward (ed.), Archaeology at ANZAAS, Canberra, pp.8695. Canberra: Canberra Archaeological Society.

Neal, R. 1984 Rescue Archaeology Near Blue Lake, North Stradbroke Island. Unpublished report to Associated Minerals Consolidated Ltd and the Archaeology Branch, Department of Community Services. St Lucia: Department of Anthropology and Sociology, University of Queensland, Brisbane.

Neal, R.A. 1986 Results of the Archaeological Inspection of Proposed Telecom DRCS and Broadband Locations for December 1986. Unpublished report to Telecom Australia.

Neal, R. 1989 Results of National Estate Funding of Radiocarbon Dates from Archaeological Sites on North Stradbroke Island. Unpublished report to the Cultural Heritage Section, Queensland Department of Community Services and Ethnic Affairs, Brisbane.

Neal, R. and E. Stock 1986 Pleistocene occupation in the south-east Queensland coastal region. Nature 323:618-
621.

Nolan, A. 1986 Sandstone Point: Temporal and Spatial Patterns of Aboriginal Site Use at a Midden Complex, South-East Queensland. Unpublished B.A. (Hons) thesis, Department of Anthropology and Sociology, University of Queensland, Brisbane.

Novello, M. 1989 A Taphonomic Analysis of the Faunal Remains from Platypus Rockshelter (Site KB:A70), S.E. Queensland. Unpublished B.A. (Hons) thesis, Department of Anthropology and Sociology, University of Queensland, Brisbane.

Oakley, K.P., B.G. Campbell and T.I. Molleson 1975 Catalogue of Fossil Hominids: Americas, Asia, Australasia. Vol. 3. London: British Museum (Natural History).

Palmerston, C. 1887 The diary of a northern pioneer. The Queensland Figaro 12 February-23 April 1887, pp.265-266, 295, 346, 351, 385, 433, 467, 491, 545$546,596,651$.

Pardoe, C. 1993 The Pleistocene is still with us: Analytical constraints and possibilities for the study of ancient human remains in archaeology. In M.A. Smith, M. Spriggs and B. Fankhauser (eds), Sahul in Review: Pleistocene Archaeology in Australia, New Guinea and Island Melanesia, pp.81-94. Occasional Papers in Prehistory 24. Canberra: Department of Prehistory, Australian National University.

Pearson, W. 1989 A technological analysis of stone artefacts from Yam Camp surface scatter and rockshelter, S.E. Cape York Peninsula. Queensland Archaeological Research 6:91-102.

Pearson, W. 1990 Prehistoric Aboriginal Land and Resource Use in Southeast Cape York Peninsula: A Technological View. Unpublished B.A. (Hons) thesis, Department of Archaeology and Palaeoanthropology, University of New England, Armidale.

Pentecost, P. and M. Bird 1995 Report on a Cultural Resource Management and Site Stabilisation Project at Kennedy A Rockshelter, Kennedy Valley, North Queensland. Unpublished report to the Jumbun and CAMU Aboriginal Communities.

Polach, H.A., J.J. Stipp, J. Golson and J.F. Lovering 1967 ANU radiocarbon date list I. Radiocarbon 9:15-27.

Quinnell, M.C. 1972 Aboriginal rock engravings near Rocky Scrub Creek, Junction View, southeast Queensland: Tyron's Pigeon Creek site re-recorded. Memoirs of the Queensland Museum 16(2):215-221.

Quinnell, M.C. 1975 Aboriginal rock art in Carnarvon Gorge south-central Queensland. Anthropological Society of Queensland Newsletter 69:2-10.

Quinnell, M.C. 1976 Aboriginal Rock Art in Carnarvon Gorge, South Central Queensland. Unpublished MA thesis, Department of Prehistory and Archaeology, University of New England, Armidale.

Quinnell, M.C. 1977 Schematisation and naturalism in the rock art of south central Queensland. In P.J. Ucko (ed.), Form in Indigenous Art: Schematisation in the Art of 
Aboriginal Australia and Prehistoric Europe, pp.414417. Canberra: Australian Institute of Aboriginal Studies.

Rafter, T.A., N.W.G. Macintosh and E.D. Gill 1970 The dating of man in Australia. In N.G. Kaigi (ed.), Abstracts of the International Symposium on Hydrogeochemistry and Biogeochemistry, Toyko. Tokyo: International Association of Geochemistry and Cosmochemistry.

Rafter, T.A., N.W.G. Macintosh and E.D. Gill 1973 The dating of man in Australia. In N.G. Kaigi (ed.), International Symposium on Hydrogeochemistry and Biogeochemistry, Toyko. Washington, D.C.: Clarke.

Redmond, K.B. 1963 Unique Aboriginal stencil in Queensland. Australian Natural History 14(8):271.

Reid, J. 1997 Results and Analysis of E1: An Investigation of the Archaeological Record of the Eurimbula Shell Midden Complex, Central Queensland Coast. Unpublished report submitted for ID232: Independent Project in Aboriginal and Torres Strait Islander Studies I, Aboriginal and Torres Strait Islander Studies Unit, University of Queensland, Brisbane.

Reid, J. 1998 An Archaeological Approach to Quarry Studies: A Technological Investigation of the Ironbark Site Complex, Southern Curtis Coast, Australia. 2 vols. Unpublished B.A. (Hons) thesis, Department of Anthropology and Sociology, University of Queensland, Brisbane.

Reid, J. 1999 Thesis Abstract: An Archaeological Approach to Quarry Studies: A Technological Investigation of the Ironbark Site Complex, Southern Curtis Coast, Australia. Australian Archaeology 48:58.

Reimer, P. 2000 Marine reservoir correction database. http://calib.org/marine/

Richardson, N. 1984 An archaeological investigation of sandmining lease SML 931 on North Stradbroke Island. In R.J. Coleman, J. Covacevich and P. Davie (eds), Focus on Stradbroke: New Information on North Stradbroke Island and Surrounding Areas, 1974-1984, pp.23-32. Brisbane: Boolarong Publications.

Richardson, N. 1992 Conjoin sets and stratigraphic integrity in a sandstone shelter: Kenniff Cave (Queensland, Australia). Antiquity 66:408-418.

Richardson, N. 1996 Seeing is believing: A graphical illustration of the vertical and horizontal distribution of conjoined artefacts using DesignCAD 3D. In S. Ulm, I. Lilley and A. Ross (eds), Australian Archaeology 95: Proceedings of the 1995 Australian Archaeological Association Annual Conference, pp.8195. Tempus 6. St Lucia: Anthropology Museum, Department of Anthropology and Sociology, University of Queensland.

Richter, J. 1994 A Pound of Bungwall and Other Measures. Unpublished B.A. (Hons) thesis, Department of Anthropology and Sociology, University of Queensland, Brisbane.

Richter, J. 1996 Weights and measures: Estimating food consumption from use-wear analysis. In S. Ulm, I.
Lilley and A. Ross (eds), Australian Archaeology '95: Proceedings of the 1995 Australian Archaeological Association Annual Conference, pp.161-168. Tempus 6. St Lucia: Anthropology Museum, Department of Anthropology and Sociology, University of Queensland.

Ridges, M. 1995 An Investigation into the Aboriginal Rock Art Paints of the Selwyn Ranges, Northwest Queensland. Unpublished B.Sc (Hons) thesis, Department of Archaeology and Palaeoanthropology, University of New England, Armidale.

Ridges, M., I. Davidson and D. Tucker 2000 The organic environment of paintings on rock. In G.K. Ward and C. Tuniz (eds), Advances in Dating Australian RockMarkings: Papers from the First Australian RockPicture Dating Workshop, pp.61-70. Occasional AURA Publication 10. Melbourne: Australian Rock Art Research Association.

Ritchie, A. 1979 The Talgai Skull story and the use - or misuse - of archives. The Artefact 4(1):41-49.

Robins, R.P. 1983 This Widow Land: An Evaluation of Public Archaeology in Queensland using Moreton Island as a Case Study. Unpublished MA thesis, Department of Anthropology and Sociology, University of Queensland, Brisbane.

Robins, R.P. 1984 The excavation of three archaeological sites on Moreton Island: First Ridge and the Little Sandhills. Queensland Archaeological Research 1:5160.

Robins, R.P. 1993 Archaeology and the Currawinya Lakes: Towards a Prehistory of Arid Lands of Southwest Queensland. Unpublished $\mathrm{PhD}$ thesis, Faculty of Environmental Sciences, Griffith University, Brisbane.

Robins, R.P. 1995 The results of test excavations in three rockshelters in southwest Queensland. Memoirs of the Queensland Museum 38(2):643-666.

Robins, R.P. 1998 Archaeological investigations at Youlain Springs, southwest Queensland. Memoirs of the Queensland Museum, Cultural Heritage Series 1(1):57-74.

Robins, R.P., E.C. Stock and D.S. Trigger 1998 Saltwater people, saltwater country: Geomorphological, anthropological and archaeological investigations of the coastal lands in the southern gulf country of Queensland. Memoirs of the Queensland Museum, Cultural Heritage Series 1(1):75-125.

Roche, N. 1999 Reading the Bones: An Analysis of Cultural and Palaeoenvironmental Trends at Hay Cave, S.E. Cape York. Unpublished B.A. (Hons) thesis, Department of Sociology, Anthropology and Archaeology, University of Queensland, Brisbane.

Rosenfeld, A. 1975 The Early Man sites: Laura, 1974. Australian Institute of Aboriginal Studies Newsletter (N.S.) 3:37-40.

Rosenfeld, A., D. Horton and J. Winter 1981 Early Man in North Queensland: Art and Archaeology in the Laura Area. Terra Australis 6. Canberra: Department of Prehistory, Research School of Pacific Studies, 
Australian National University.

Ross, A. 2000 Results of the Lazaret Midden Excavation on Peel Island in a Landscape and Seascape Context. Unpublished paper presented to the Department of Sociology, Anthropology and Archaeology, University of Queensland, Brisbane, 19 May 2000.

Ross, A. and S. Coghill 1996 The Creation of Evidence: Sieve Size and the Evidence for Fishing in the Lazaret Midden, Peel Island, Moreton Bay. Unpublished paper presented to the Australian Archaeological Association Annual Conference, Dzintari, South Australia, 6 December 1996.

Ross, A. and R. Duffy 2000 Fine mesh screening of midden material and the recovery of fish bone: The development of flotation and deflocculation techniques for an efficient and effective procedure. Geoarchaeology 15(1):21-41.

Rowe, C. 1998 The Value of Land Snails for Reconstruction of Palaeoenvironments in Northern Queensland. Unpublished B.Sc. thesis, Department of Geography and Environmental Science, Monash University, Melbourne.

Rowe, C., B. David, J. Stanisic and H. Lourandos in press The Helicinid land snail Pleuropoma extincta (Odhner, 1917) as an environmental indicator in archaeology. Memoirs of the Queensland Museum.

Rowland, M.J. 1980 The Keppel Islands - Preliminary investigations. Australian Archaeology 11:1-17.

Rowland, M.J. 1981 Radiocarbon dates for a shell fishhook and disc from Mazie Bay, North Keppel Island. Australian Archaeology 12:63-69.

Rowland, M.J. 1982a Stockyard Point - Capricorn Coast: A brief note from terra incognita. Australian Archaeology 15:35-42.

Rowland, M.J. 1982b Further radiocarbon dates from the Keppel Islands. Australian Archaeology 15:43-48.

Rowland, M.J. 1983 A note on corrections to radiocarbon dates from the Keppel Islands. Australian Archaeology 17:134-135.

Rowland, M.J. 1984 A long way in a bark canoe: Aboriginal occupation of the Percy Isles. Australian Archaeology 18:17-31.

Rowland, M.J. 1985a Further radiocarbon dates from Mazie Bay, North Keppel Island. Australian Archaeology 21:113-118.

Rowland, M.J. 1985b Archaeological investigations on Moa and Naghi Islands, western Torres Strait. Australian Archaeology 21:119-132.

Rowland, M.J. 1986 The Whitsunday Islands: Initial historical and archaeological observations and implications for future work. Queensland Archaeological Research 3:72-87.

Rowland, M.J. 1987 Preliminary Archaeological Survey of Coastal Areas of the Bundaberg 1:250,000 sheet (KE). Unpublished report to the Queensland Department of Environment and Heritage, Brisbane.
Rowland, M.J. 1992 Conservation Plan for Cultural Heritage Sites on the Keppel Island Group, Central Queensland. Unpublished report to the Livingstone Shire Council and National Parks and Wildlife Branch, Division of Conservation, Department of Environment and Heritage, Brisbane.

Rowland, M.J. 1996 Prehistoric archaeology of the Great Barrier Reef province - Retrospect and prospect. In P. Veth and P. Hiscock (eds), Archaeology of Northern Australia, pp.191-212. Tempus 4. St Lucia: Anthropology Museum, Department of Anthropology and Sociology, University of Queensland.

Rowland, M.J. 1999 The Keppel Islands - 'a 3000 year' event revisited. In J. Hall and I.J. McNiven (eds), Australian Coastal Archaeology, pp.141-155. Research Papers in Archaeology and Natural History 31. Canberra: ANH Publications, Department of Archaeology and Natural History, Research School of Pacific and Asian Studies, Australian National University.

Sale, K.M. and B.C. Barker 1992 The Nara Inlet Aboriginal Art: Assessment and Recommendations Relating to Provenance, Conservation and Management. Unpublished report to the Queensland National Parks and Wildlife Service.

Sale, K.M. and A. Watchman 1993 Pigment Analysis at Nara Inlet Rock Art Site, Hook Island, Whitsunday Group, Far North Queensland. Unpublished report to the Australian Institute of Aboriginal and Torres Strait Islander Studies and Queensland National Parks and Wildlife Service.

Skelton, S.M. 1996 Starch Trek: The Search for Residue: An Examination of the Relationship Between Technological Change and Resource Exploitation at Platypus Rockshelter(KBA70), Southeast Queensland. Unpublished PGDipArts (Anthropology) thesis, Department of Anthropology and Sociology, University of Queensland, Brisbane.

Small, M.W. 1992 Gulumba's Land: A Study in Ethnoarchaeology. Unpublished B.A. (Hons) thesis, Department of Behavioural Science, James Cook University of North Queensland, Townsville.

Smith, A.D. 1992 An Archaeological Site Location and Subsistence-Settlement Analysis of Bribie Island, Southeast Queensland. Unpublished B.A. (Hons) thesis, Department of Anthropology and Sociology, University of Queensland, Brisbane.

Smith, M.A. 1985 A morphological comparison of central Australian seedgrinding implements and Australian Pleistocene-age grindstones. The Beagle: Occasional Papers of the Northern Territory Museum of Arts and Sciences 2:23-38.

Smith, M.A. 1986 The antiquity of seedgrinding in arid central Australia. Archaeology in Oceania 21(1):29-39.

Smith, M.A. and N.D. Sharp 1993 Pleistocene sites in Australia, New Guinea and Island Melanesia: Geographic and temporal structure of the archaeological record. In M.A. Smith, M. Spriggs and 
B. Fankhauser (eds), Sahul in Review: Pleistocene Archaeology in Australia, New Guinea and Island Melanesia, pp.37-59. Occasional Papers in Prehistory 24. Canberra: Department of Prehistory, Research School of Pacific Studies, Australian National University.

Smith, P., T. Brown and W.B. Wood 1981 Tooth size and morphology in a recent Australian Aboriginal population from Broadbeach, south east Queensland. American Journal of Physical Anthropology 55:423432.

Smith, S.A. 1918 The fossil human skull found at Talgai, Queensland. Royal Society of London: Philosophical Transactions Series B, 208:331-387.

Solomon, S. 1986 A taphonomic analysis of the fauna from Gatton Rock Shelter (Squares G4c and G4d). In M. Morwood, The archaeology of art: Excavations at Maidenwell and Gatton Shelters, southeast Queensland. Queensland Archaeological Research 3:88-132.

Stephens, K. 1990 A late Quaternary vegetation history from south east Cape York derived from swamp and archaeological sediments. Unpublished B.Environ.Sc (Hons) thesis, Department of Geography, University of Wollongong, Wollongong.

Stockton, J. 1981 Radiocarbon dates for archaeological sites in Tasmania. Australian Archaeology 12:97-101.

Stone, T. 1989 Origins and environmental significance of shell and earth mounds in northern Australia. Archaeology in Oceania 24(2):59-64.

Stone, T. 1991 Two birds with one Stone: A reply. Archaeology in Oceania 26(1):26-28.

Stone, T. 1992 Origin of the Weipa Shell Mounds. Unpublished MSc thesis, Department of Geography, Australian National University, Canberra.

Stone, T. 1993 Birds, boffins and blunders. Australian Geographic 29:25-26.

Stuiver, M. and H.A. Polach 1977 Discussion: Reporting of ${ }^{14} \mathrm{C}$ data. Radiocarbon 19(3):355-363.

Stuiver, M. and P.J. Reimer 1993 Extended ${ }^{14} \mathrm{C}$ data base and revised CALIB $3.0{ }^{14} \mathrm{C}$ age calibration program. Radiocarbon 35(1):215-230.

Stuiver, M., P.J. Reimer, E. Bard, J.W. Beck, G.S. Burr, K.A. Hughen, B. Kromer, G. McCormac, J. van der Plicht and M. Spurk 1998 INTCAL98 Radiocarbon age calibration, 24,000-0 cal BP. Radiocarbon 40(3):10411083.

Sutton, S.A. 1990 Pleistocene axes in Sahul: A response to Morwood and Tresize. Queensland Archaeological Research 7:95-109.

Taylor, R.E. 1987 Radiocarbon Dating: An Archaeological Perspective. New York: Academic Press.

Taylor, R.E. and M.J. Aitken (eds) 1997 Chronometric Dating in Archaeology. Advances in Archaeological and Museum Science 2. New York: Plenum Press.

Temple, R.B. and M. Barbetti 1981 Revisions to radiocarbon dates from the Sydney University
Radiocarbon Laboratory. Australian Archaeology 13:28-29.

Terlich, N.J. 1998 Teeth, Bones, and Other Indices: Interpreting a Terminal Pleistocene Faunal Assemblage of Hay Cave, southeast Cape York Peninsula, north Queensland. Unpublished PGDipArts (Anthropology) thesis, Department of Anthropology and Sociology, University of Queensland, Brisbane.

Thom, B.G., G.M. Bowman, R. Gillespie, R. Temple and M. Barbetti 1981 Radiocarbon Dating of Holocene Beach-Ridge Sequences in South-East Australia. Monograph 11. Duntroon, ACT: Department of Geography, University of New South Wales, Royal Military College.

Trezise, P.J. 1971 Rock Art of South-East Cape York. Canberra: Australian Institute of Aboriginal Studies.

Tugby, D. and E. Tugby 1965 An Aboriginal kitchenmidden near Caloundra, south-east Queensland. Mankind 6(5):197-201.

Tyron, H. 1884 On an undescribed class of rock drawings of Aborigines in Queensland. Proceedings of the Royal Society of Queensland 1:45-52.

Ulm, S.G.F. 1995 Fishers, Gatherers and Hunters on the Moreton Fringe: Reconsidering the Prehistoric Aboriginal Marine Fishery in Southeast Queensland, Australia. Unpublished B.A. (Hons) thesis, Department of Anthropology and Sociology, University of Queensland, Brisbane.

Ulm, S. 2000 Evidence for early focussed marine resource exploitation from an open coastal site in central Queensland. Australian Archaeology 51:66-67.

Ulm, S. in press The Seven Mile Creek Mound: New evidence for mid-Holocene Aboriginal marine resource exploitation in central Queensland. Proceedings of the Royal Society of Queensland 110.

Ulm, S. in prep. Marine and estuarine reservoir effects in central Queensland, Australia: Determination of $\Delta \mathrm{R}$ values. To be submitted to Geoarchaeology.

Ulm, S., B. Barker, A. Border, J. Hall, I. Lilley, I. McNiven, R. Neal and M. Rowland 1995 Pre-European coastal settlement and use of the sea: A view from Queensland. Australian Archaeology 41:24-26.

Ulm, S., M. Carter, J. Reid and I. Lilley 1999a Eurimbula Site 1, Curtis Coast: Site report. Queensland Archaeological Research 11:105-122.

Ulm, S., T. Eales and S. L'Estrange 1999b Post-European Aboriginal occupation of the southern Curtis Coast, central Queensland. Australian Archaeology 48:42-43.

Ulm, S. and J. Hall 1996 Radiocarbon and cultural chronologies in southeast Queensland prehistory. In S. Ulm, I. Lilley and A. Ross (eds), Australian Archaeology '95: Proceedings of the 1995 Australian Archaeological Association Annual Conference, pp.4562. Tempus 6. St Lucia: Anthropology Museum, Department of Anthropology and Sociology, University of Queensland.

Ulm, S. and I. Lilley 1999 The archaeology of the southern 
Curtis Coast: An overview. Queensland Archaeological Research 11:59-84.

Urry, J. 1978 Old questions: New answers? Thoughts on the origin and antiquity of man in Australia. Aboriginal History 2(2):149-166.

Vanderwal, R.L. 1973 The Torres Strait: Protohistory and beyond. In P. Lauer (ed.), Occasional Papers in Anthropology 2:157-194. St Lucia: Anthropology Museum, University of Queensland.

Veitch, B. 1999 Shell middens on the Mitchell Plateau: A reflection of a wider phenomenon? In J. Hall and I.J. McNiven (eds), Australian Coastal Archaeology, pp.51-64. Research Papers in Archaeology and Natural History 31. Canberra: ANH Publications, Department of Archaeology and Natural History, Research School of Pacific and Asian Studies, Australian National University.

Vernon, K. 1999 Post-Contact Cultural Continuity in Central Queensland: Bottle Bases and Retouched Glass Artefacts. Unpublished report submitted for ID233: Independent Project in Aboriginal and Torres Strait Islander Studies II, Aboriginal and Torres Strait Islander Studies Unit, University of Queensland, Brisbane.

Walsh, G.L. 1985 Archaeological Significance of Hook Island. Unpublished report to Queensland National Parks and Wildlife Service.

Walsh, G.L. 1988 Australia's Greatest Rock Art. Sydney: E.J. Brill-Robert Brown and Associates.

Walters, I.N. 1979 Information Loss in Zooarchaeology: A Study of Vertebrate Remains from Two Middens, Moreton Island, Queensland. Unpublished B.A. (Hons) thesis, Department of Anthropology and Sociology, University of Queensland, Brisbane.

Walters, I.N. 1980 Vertebrate remains from two sites on Moreton Island, Queensland. Australian Archaeology 11:28-36.

Walters, I.N. 1986 Another Kettle of Fish: The Prehistoric Moreton Bay Fishery. Unpublished PhD thesis, Department of Anthropology and Sociology, University of Queensland, Brisbane.

Walters, I. 1989 Intensified fishery production at Moreton Bay, southeast Queensland, in the late Holocene. Antiquity 63:215-224.

Walters, I. 1992a Farmers and their fires, fishers and their fish: Production and productivity in pre-European south-east Queensland. Dialectical Anthropology 17:167-182.

Walters, I. 1992b Seasonality of fishing in south-east Queensland. Queensland Archaeological Research 9:29-34.

Walters, I. 1992c Antiquity of marine fishing in south-east Queensland. Queensland Archaeological Research 9:35-37.

Walters, I.N. and J. Hall 1980 Another fish species from Moreton Island Aboriginal middens: The tailor, Pomatomus saltatrix. In P.K. Lauer (ed.), Occasional
Papers in Anthropology 10:113-114. St Lucia: Anthropology Museum, University of Queensland.

Walters, I., P. Lauer, A. Nolan, G. Dillon and M. Aird 1987 Hope Island: Salvage excavation of a Kombumerri site. Queensland Archaeological Research 4:80-95.

Ward, I., C. Patterson and A. Watchman 1999 The environment and condition of multi-layered Aboriginal paintings at Kennedy-A in the wet tropics, north Queensland. Rock Art Research 16(1):25-35.

Ward, W.T. and J.L.F. Hacker 1981 Sedimentation and Soil Development at Brisbane Airport. Unpublished report to the CSIRO, Brisbane.

Ward, W.T. and J.L.F. Hacker 1982 Sedimentation and soil development at Brisbane Airport. Sedimentology Newsletter 11:54.

Ward, W.T. and I.P. Little 2000 Sea-rafted pumice on the Australian east coast: Numerical classification and stratigraphy. Australian Journal of Earth Sciences 47:95-109.

Watchman, A. 1990 A summary of occurrences of oxalaterich crusts in Australia. Rock Art Research 7(1):44-50.

Watchman, A.L. 1993 Evidence of a 25,000-year-old pictograph in northern Australia. Geoarchaeology 8(6):465-473.

Watchman, A. 2000 Micro-excavation and laser extraction methods for dating carbon in silica skins and oxalate crusts. In G.K. Ward and C. Tuniz (eds), Advances in Dating Australian Rock-Markings: Papers from the First Australian Rock-Picture Dating Workshop, pp.3539. Occasional AURA Publication 10. Melbourne: Australian Rock Art Research Association.

Watchman, A. and N. Cole 1993 Accelerator radiocarbon dating of plant-fibre binders in rock paintings from northeastern Australia. Antiquity 67:355-358.

Watchman, A. and E. Hatte 1996 A nano approach to the study of rock art: 'The Walkunders', Cillagoe, north Queensland, Australia. Rock Art Research 13(2):85-92.

Webb, E. 1992 Sand traps for the unwary - Problems in the interpretation of sedimentological analyses. Queensland Archaeological Research 9:43-49.

Westcott, C. 1997 A Technological Analysis of the Stone Assemblage from Big Foot Art Site, Cania Gorge. Unpublished B.A. (Hons) thesis, Department of Anthropology and Sociology, University of Queensland, Brisbane.

Westcott, C. 1999 Thesis Abstract: A Technological Analysis of Stone Artefacts from Big Foot Art Site, Cania Gorge, Central Queensland. Australian Archaeology 48:57.

Westcott, C., I. Lilley and S. Ulm 1999a The archaeology of Cania Gorge: An overview. Queensland Archaeological Research 11:15-28.

Westcott, C., I. Lilley, S. Ulm, C. Clarkson and D. Brian 1999b Big Foot Art Site, Cania Gorge: Site report. Queensland Archaeological Research 11:43-58. 
White, J.P. 1975 Anthropological theses in Australia: A first listing (to 1974). Mankind 10(2):1-20 (Supplement).

White, J.P. 1994 Theses about prehistoric archaeology and associated disciplines in Australia, 1975-1993. Archaeology in Oceania 29(2):95-106.

Wood, W.B. 1968 An Aboriginal burial ground at Broadbeach, Queensland: Skeletal material. Mankind 6(12):681-686.

Wood, W.B. 1977 Identification of human bone from excavations at Herveys Range, Jourama and Kennedy. In H.C. Brayshaw, Aboriginal Material Culture in the Herbert/Burdekin District, North Queensland, pp.680715. Unpublished PhD thesis, James Cook University of North Queensland, Townsville.

Wood, W.B. and A.M.C. Murphy 1978 The Broadbeach Aboriginal femur - Metrical features. Journal of Anatomy 126:634.

Wood, W.B. and A.M.C. Murphy 1979 The Broadbeach Aboriginal burial site, southeast Queensland: The metrical features of the tibiae. Journal of Anatomy 128:663.

Wood-Jones, F. 1944 The antiquity of man in Australia. Nature 153:211-212.
Woolford, N.R.J. 2000 The End of the Holocene Stillstand was no Stand Still for the Gooreng Gooreng: So Why Did They Stop?: A Preliminary Investigation of Square A, the Seven Mile Creek Mound: An Analysis of Species Diversity and Intra-Specific Size of Shellfish Recovered. Unpublished report submitted for ID233: Independent Project in Aboriginal and Torres Strait Islander Studies II, Aboriginal and Torres Strait Islander Studies Unit, University of Queensland, Brisbane.

Woolston, F. 1965 An Excavation in 'Platform Gallery' near Cooktown, N.Q. Unpublished report to the Australian Institute of Aboriginal Studies, Canberra.

Woolston, F.P. and P.J. Trezise 1969 Petroglyphs of Cape York Peninsula. Mankind 7(2):120-127.

Wright, R.V.S. 1963 Report by R.V.S. Wright on Archaeological Reconnaissance Work in Cape York. Unpublished report to the Australian Institute of Aboriginal Studies Interim Council Meeting, 13 December 1963.

Wright, R.V.S. 1971 Prehistory in the Cape York Peninsula. In D.J. Mulvaney and J. Golson (eds), Aboriginal Man and Environment in Australia, pp.133140. Canberra: Australian National University Press. 